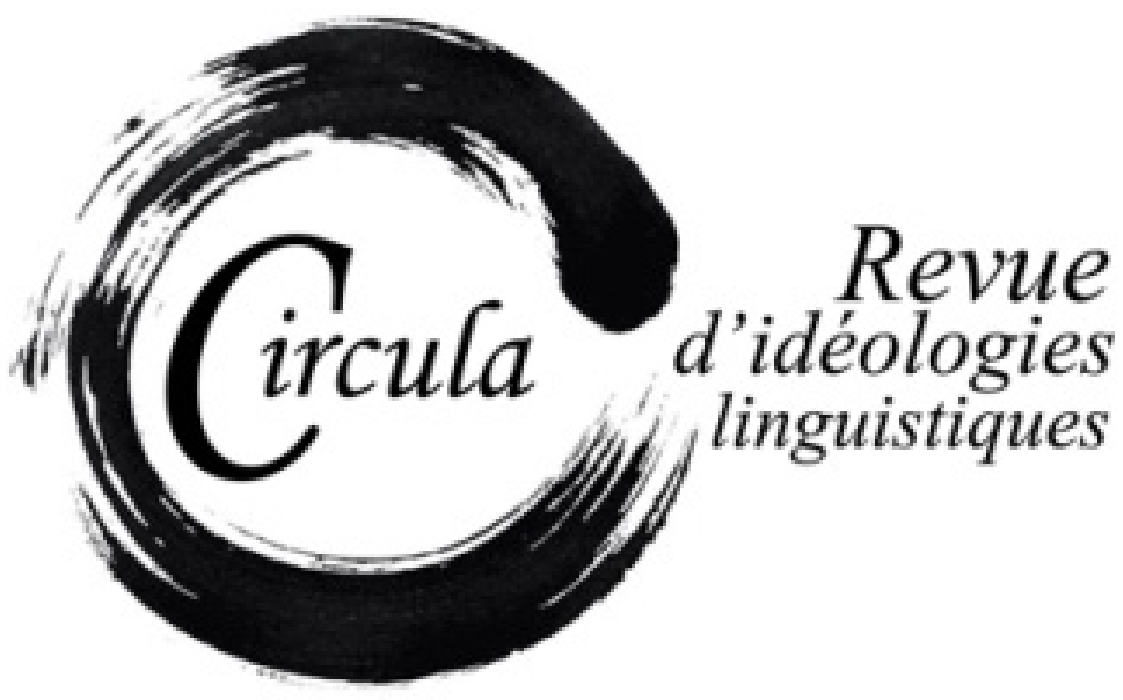

Publication: Circula, numéro 12 : LA VUlnÉRABILITÉ linguISTIQUe

Directrice: Claudia ToRRES CASTILlo

PAGES: $1-208$

ISSN: 2369-6761

URI: HTTP://HDL.HANDLE.NET/11143/18440

DOI: HTTPS://DOI.ORG/10.17118/11143/18440 


\section{Table des matières}

Présentation

Claudia Torres Castillo

Introduction : vulnérabilité linguistique, inégalités, discriminations 8

Philippe Blanchet

\section{Axe 1 : Plurilinguisme, colonisation et glottophobie: l'exemple du Mexique}

Vulnerabilidad de la lengua en hablantes indígenas, el caso de México

Leonor Delgadillo, José Arce y Susana Pastrana

Hiérarchies imaginées des locuteurs et des langues-cultures au Mexique.....

Claudia Torres Castillo

"La gente se ríe de nosotros porque hablamos otra lengua":

discriminación étnica y lingüística en León, Guanajuato, México 66

Sophia Schnuchel, David Charles Wright

\section{Axe 2 : Habiter une langue, habiter entre les langues}

Dissocier allophonie et vulnérabilité : de la nécessité d'interroger les catégories ..... 88 Maxime Alais, Silvia Lucchini, Salima El Karouni, Aphrodite Maravelaki, Élodie Oger

Les expériences artistiques en contexte plurilingue :

une mise à l'épreuve de la vulnérabilité linguistique? 108

Maud Sérusclat-Natale

Jóvenes de Telebachillerato y alfabetismos digitales.

Entre el acceso y la desigualdad.

María Guadalupe Velasco Giles, José Federico Benítez Jaramillo 


\section{Axe 3 : L'insécurité linguistique des enseignants de français langue étrangère}

L'enseignant de langue étrangère et rapport à la langue :

comment l'enseignant indien de FLE négocie l'ambivalence

"sécurité-insécurité linguistique ».

153

Vasumathi Badrinathan

Insécurité linguistique chez les enseignants non natifs de FLE :

le cas des Colombiens.

Claudia Rincón Restrepo

Valentin Feussi et Joanna Lorilleux (dir.), (2020), (In)sécurité linguistique en francophonies: perspectives in(ter)disciplinaires, Paris, L'Harmattan, coll. «Espaces discursifs », 452 p. [ISBN : 978-2-343-20806-0]

Cynthia Barré-Benoit

Covino, Sandra (2019), Linguistica e nazionalismo tra le due guerre mondiali, Bologna, il Mulino, 271 p. [ISBN: 978-88-15-28387-0] 206

Maria Silvia Rati 


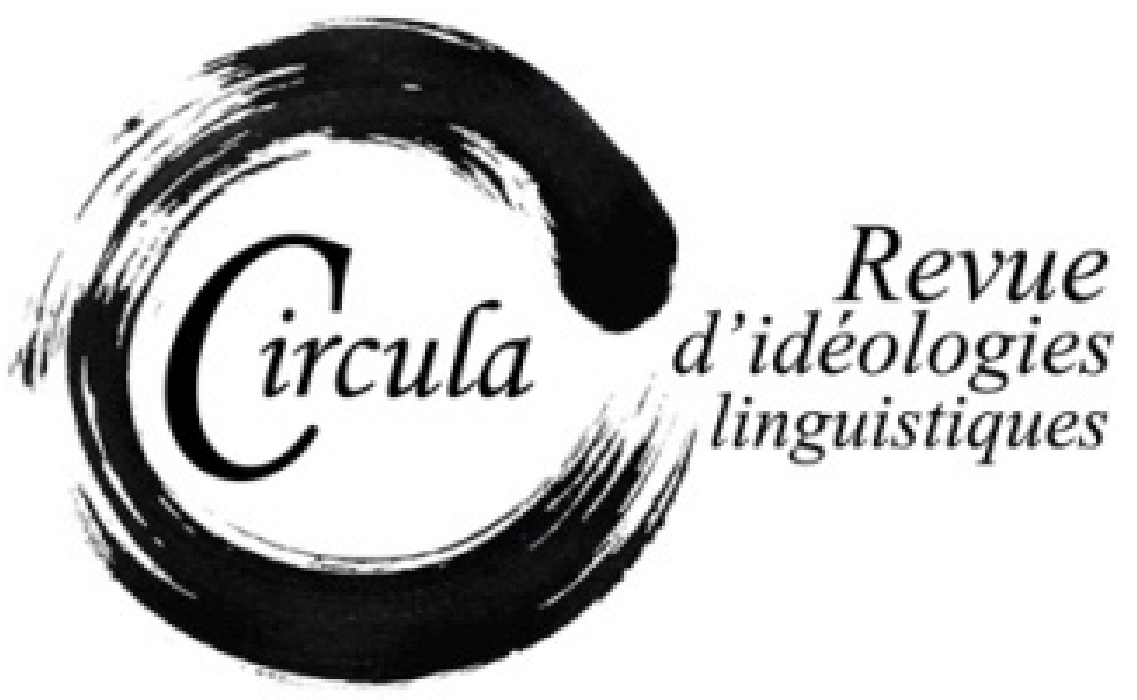

TITRE: PRÉSENTATION

Auteur: Claudia TorRes CASTILlo, Universidad AutónOma del ESTADO de MÉXICO

RevUe: CIRCula, NUMÉRO 12 : LA VULNÉRABILITÉ LINGUISTIQUE

DiRECTRICE: CLAUDIA TORRES CASTILLO

PAGES: $1-6$

ISSN: 2369-6761 


\section{Présentation}

Claudia Torres Castillo, Universidad Autónoma del Estado de México

claudiatorresc@yahoo.com.mx

La vulnérabilité est devenue un paradigme en sciences humaines et sociales. Depuis les années 1990, la thématique de la vulnérabilité peut se substituer à celles de la pauvreté et de la justice sociale (Brodiez-Dolino, 2015). La vulnérabilité est vue comme le fait d'être ou de se sentir en danger, d'être fragile, ou bien d'être soumis à différentes contraintes. Butler (2004) nous met en garde sur le pouvoir des mots sur l'autre dans les domaines personnel, académique et social. La vulnérabilité linguistique est un sujet fort intéressant et constitue un champ d'intervention pour les sociolinguistes qui peut être abordé avec d'autres notions fondamentales : la langue première vis-à-vis de la langue étrangère ou seconde de locuteurs bilingues ; les modèles stéréotypés des locuteurs «natifs » et «non natifs » d'une langue; ou bien à travers d'autres types de hiérarchies imaginées qui idéalisent les langues, les locuteurs ou l'apprentissage des langues étrangères.

On reconnait habituellement certains groupes vulnérables parmi la société : les enfants, les minorités ethniques, les victimes de catastrophes ou bien les personnes âgées. Sont aussi considérées comme des groupes fragiles les personnes handicapées et les femmes. Ces groupes vulnérables sont identifiés comme des personnes défavorisées ou des minorités en danger. Il s'agit de penser la vulnérabilité à travers les genres (femmes vs hommes) et à travers les âges (personnes âgées et enfants) ; on s'intéresse aussi aux minorités affaiblies (personnes avec des capacités différentes ou avec un handicap). De plus, les migrants sont aussi considérés comme un groupe vulnérable. Ainsi, les imaginaires sociaux des diverses personnes appartenant à ces minorités fragilisées peuvent aussi s'ajouter en tant que facteurs qui engendrent la vulnérabilité. C'est pourquoi il existe différents types de profils vulnérables, qui seraient vulnérables à différents degrés selon les interactions avec les autres, l'autre - étranger, l'autre - «natif» ou «non-natif».

Diverses situations dans mon parcours linguistique en tant que migrante en France et en tant que Mexicaine parlant français au Mexique m’ont sensibilisée à la vulnérabilité linguistique : l'idée de ce numéro thématique est en quelque sorte l'aboutissement de mes réflexions tout au long de la préparation de deux thèses de doctorat (au Mexique et en France). C'est pourquoi, en tant que sociolinguiste, je voulais m'intéresser à des questions qui me tiennent à cœur ; mais surtout transmettre mon enthousiasme pour cette thématique à d'autres chercheurs. 
Dans ce numéro de Circula, nous nous intéressons à la vulnérabilité linguistique thématisée et politisée à travers les droits de tous les individus à une éducation langagière en langue première (L1, contenus dans les droits de l'homme), et dans le respect de leurs parcours linguistiques. La vulnérabilité linguistique provient du fait d'être ou de se sentir en danger, fragile ou soumis à des contraintes lors de l'expression en langue première, ce qui pourrait provoquer des problèmes identitaires, du mépris de soi et en même temps un éloignement de l'autre, ou bien un effet de surplomb de la langue du pays d'accueil.

Comme nous venons de le mentionner, la vulnérabilité linguistique se manifeste de plusieurs manières dans différents contextes. Sa description et son analyse permettraient dans un premier temps de repérer des situations individuelles et de minorités linguistiques; et dans un deuxième temps, de comprendre la vulnérabilité linguistique lorsqu'elle apparait dans le contexte de l'apprentissage des langues étrangères. En sciences sociales et humaines, l'absence de prise en compte de l'historicité du chercheur (De Robillard, 2011) et des témoins peut mener vers une sorte d'aveuglement envers la diversité linguistique et culturelle, et surtout peut montrer un manque d'intérêt pour ce type de problématiques. Nous entendons par historicité l'ensemble des histoires, expériences et parcours en tant que locuteurs des langues premières et étrangères. C'est pourquoi nous nous sommes intéressés aux expériences individuelles des locuteurs dans des contextes différents, toujours avec une constante : le conflit. La vulnérabilité linguistique nous a permis de réunir dans un même numéro des réflexions et analyses de chercheurs d'horizons différents (psychologie, éducation, droits des langues indigènes, sociologie et didactique des langues étrangères). Nous avons regroupé divers cas de vulnérabilité linguistique à travers trois axes : $1^{\circ}$ plurilinguisme, colonisation et glottophobie : l'exemple du Mexique ; $2^{\circ}$ habiter une langue, habiter entre les langues ; $3^{\circ}$ l'insécurité linguistique des enseignants de français langue étrangère.

\section{Axe 1 : Plurilinguisme, colonisation et glottophobie : l'exemple du Mexique}

En tant que locutrice plurilingue et grâce à mon double parcours en France et au Mexique, il m'a paru nécessaire d'étudier la réalité mexicaine à travers les notions de plurilinguisme, de colonisation et de glottophobie. Cette dernière notion a particulièrement retenu notre attention. La glottophobie est, selon Blanchet (2016 : 45), «le mépris, la haine, l'agression, le rejet, l'exclusion, de personnes, discrimination négative effectivement ou prétendument fondées sur le fait de considérer incorrectes, inférieures, mauvaises certaines formes linguistiques ». II me semble alors que le Mexique est l'exemple idéal pour étudier la diversité linguistique et culturelle ainsi que d'autres problématiques sociolinguistiques en Amérique latine. Les auteurs des articles de ce premier axe se sont interrogés sur les diverses formes de glottophobie dans le contexte mexicain (même s'ils ne font pas allusion à la notion). On peut constater que les idéologies linguistiques glottophobes sont puissantes et sont bien installées au Mexique. Le colonialisme mêlé à la pérennisation d'une idéologie du monolinguisme fait surgir diverses situations de vulnérabilité linguistique parmi les individus et parmi les groupes. 
Cet axe correspond à des descriptions et analyses de situations où parler une langue (étrangère ou première) de façon « différente » entrainerait des discriminations linguistiques basées sur des façons de parler idéalisées. Ces situations provoqueraient l'exclusion d'autres langues, de la discrimination et surtout la négation de la pluralité linguistique mexicaine.

\section{Axe 2 : Habiter une langue, habiter entre les langues}

Pour un apprenant de langues, les droits linguistiques sont souvent perçus et définis uniquement autour de ses compétences langagières en langue étrangère (LE). Si bien que l'apprentissage et l'acquisition d'une nouvelle langue cherchent à augmenter ou améliorer la maitrise de cette LE, et que l'enseignement des langues à travers l'approche actionnelle forme les individus à « vivre » en langue étrangère. Souvent, les langues premières ainsi que les connaissances préalables continuent d'être négligées. Dans ce deuxième axe, nous avons voulu mettre en évidence des expériences de vie de minorités linguistiques ou d'individus dans différentes sociétés qui, d'une certaine façon, vivent en conflit entre les langues. Les auteurs y présentent des expériences d'apprenants en Belgique, en France et au Mexique et proposent des réflexions sur des parcours linguistiques de personnes qui se voient fragilisées parce qu'elles s'expriment en langue étrangère ou minoritaire. Ils s'intéressent aux stratégies utilisées par les locuteurs pour « apprendre à vivre » en langue étrangère, tout en gardant leurs attachements et leur langue première. Ils ont cherché à s'interroger sur la manière dont des locuteurs bilingues, voire plurilingues, « habitent la langue étrangère ».

Nous avons également, dans ce numéro, cherché à analyser la pertinence des dispositifs d'accueil pour les personnes migrantes. Ces dispositifs, bien qu'offrant une formation en langue, n'épargnent pas à leurs bénéficiaires le sentiment d'être seuls et incompris. Par ailleurs, souvent, l'apprentissage de la langue étrangère ne prend pas - ou trop peu - en compte les compétences transversales ou interculturelles dont les apprenants disposent. Ces individus doivent apprendre à vivre dans une autre société et à s'exprimer en continu en LE. Alors, ils mettent en place des stratégies ou des actions qui leur permettent de continuer à vivre dans leur société d'accueil. Les auteurs nous présentent des histoires de plusieurs apprenants et utilisateurs de langues étrangères qui, d'une certaine façon, habitent entre les langues ou « entre deux mères » (Coste, 2003).

\section{Axe 3 : L'insécuritếlinguistique des enseignants de français langue étrangère}

La vulnérabilité peut aussi se traduire par une certaine fragilité linguistique en langue étrangère : la faible maitrise de la langue étrangère fragiliserait les individus. Mais dans tous les cas, l'insécurité linguistique est l'état d'esprit ressenti par un locuteur lorsqu'il considère sa façon de parler comme peu valorisante, la comparaison étant établie à travers un modèle plus prestigieux que ce locuteur ne pratique pas (Calvet, 1993). Les individus expérimentent de l'insécurité linguistique parce qu'ils se comparent à « un modèle mythique et inaccessible» (Francard, 1993 : 13). Les situations d'insécurité 
linguistique se multiplient à cause des imaginaires sociaux qui renforceraient une image fantasmée d'un type de locuteur d'une certaine langue.

Les auteures des articles de cet axe se sont intéressées à des situations d'insécurité linguistique parmi des enseignants en Colombie et en Inde; deux pays avec deux langues premières différentes, mais avec une diversité linguistique incontestable. Le lecteur pourra découvrir comment certains enseignants de français langue étrangère dans ces pays se sentent en insécurité linguistique vis-à-vis de certains locuteurs. Les auteures se sont intéressées aux expressions d'insécurité qui se trouvent ancrées dans une expérience affective personnelle lors de l'exercice en tant qu'enseignant.

Le recueil d'articles de ce numéro de Circula représente à peine une esquisse collective du besoin urgent de s'intéresser à l'étude de la vulnérabilité linguistique et ses différentes formes.

Je voudrais conclure la présentation de ce numéro thématique avec plusieurs remerciements. Un grand merci aux chercheurs qui ont participé comme lecteurs et évaluateurs des articles. Au Mexique: Magdalena Hernández, Pilar Ortiz Lovillo, Bruno Baronnet, Celia Contreras et Cristina Kleinert de l'université de Veracruz; Ana Lidia Munguía Duarte de l'Université de Sonora ; David Charles Wright de l'Université de Guanajuato ; Venancio Carmona de l'Université Pédagogique Nationale et Leonor Delgadillo Guzmán de l'Université de l'État de Mexico (UAEM). En Belgique, Sylvia Luccini et Michel Francard de l'Université catholique de Louvain. En Grèce, Maria Roussi de l'International Hellenic University. Et en France, Martine Derivry de l'Université de Bordeaux; Maïtena Armagnague-Roucher et Isabelle Rigoni de l'Université Paris Lumières; Nathalie Auger, de l'Université Paul Valery de Montpellier et Gudrun Ledegen, de l'Université de Rennes. Merci pour votre temps! Sans votre participation, ce numéro thématique aurait été moins réussi. Nous avons tous travaillé au milieu de la pandémie tout au long de l'année 2020 et malgré des surcharges de travail généralisées, nous avons réussi. J'apprécie la générosité de Philippe Blanchet qui a accepté de rédiger la préface de ce numéro et qui m’a épaulée dans cette première expérience de coordination. Enfin, j'ai une pensée pleine de reconnaissance inspirée par la confiance que la revue Circula, à travers Wim Remysen, m’a accordée. 


\section{Références}

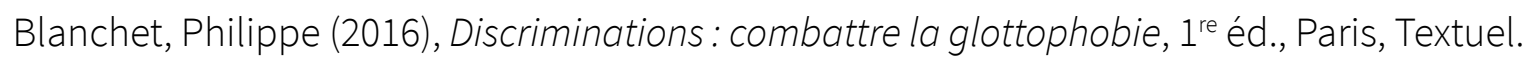

Brodiez-Dolino, Axelle (2015), «La vulnérabilité, nouvelle catégorie de l'action publique », Informations sociales, vol. 2, n 188, p. 10-18.

Butler, Judith (2004), Le pouvoir des mots : politique du performatif, Paris, Éditions Amsterdam.

Calvet, Louis-Jean (1993), La sociolinguistique, Paris, Presses universitaires de France.

Coste, Daniel (2003), «Entre mythe linguistique et politique des langues : d'une langue maternelle à l'autre », dans Daniel Coste, Danièle Moore, Mary Dalrymple et Brigitte Helmling (dir.), Les langues maternelles dans l'enseignement des langues étrangères, Lyon, ENS Éditions, p. 15-30.

De Robillard, Didier (2011), "Les vicissitudes et tribulations de "Comprendre" : un enjeu en didactique des langues et cultures? », dans Philippe Blanchet et Patrick Chardenet (dir.), Guide pour la recherche en didactique des langues et des cultures. Aproches contextualisées, Paris, Archives C, p. 21-29.

Francard, Michel, Joëlle Lambert et Françoise Berdal-Masuy (1993), L'insécurité linguistique en Communauté française de Belgique, Bruxelles, Service de la langue française de la Communauté française. 


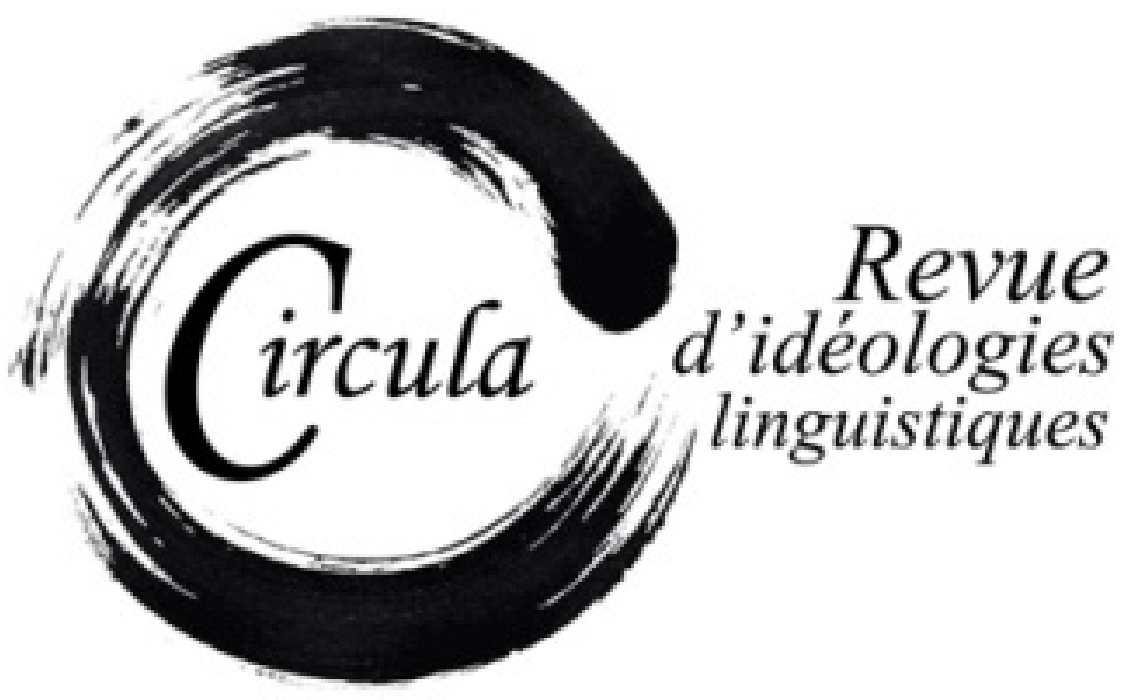

TITRE: INTRODUCTION : VULNÉRABILITÉ LINGUISTIQUE, INÉGALITÉS, DISCRIMINATIONS Auteur: PhILIPPE BLANCHET, Université RenNeS 2

Revue: Circula, NumÉro 12 : LA VULNÉRABILITÉ LINGUISTIQUE

DiRECTRICE: CLAUdia TORRES CASTILLO

PAGES: $7-17$

ISSN: 2369-6761 


\section{Introduction : vulnérabilité linguistique, inégalités, discriminations}

\section{Réflexions à partir des terrains et des analyses présentés dans ce volume}

Philippe Blanchet, Université Rennes 2 philippe.blanchet@univ-rennes2.fr

\section{Situer la notion de vulnérabilité dans le fil des recherches en sociolinguistique}

Les travaux en sociolinguistique ont construit, dès leurs débuts dans les années 1960 et jusqu'à aujourd'hui, toute une série de concepts opératoires corrélés pour rendre compte d'un ensemble de phénomènes sociaux et linguistiques que je propose de regrouper commodément sous le chapeau « inégalités sociales à prétextes linguistiques » : diglossie, insécurité linguistique, glottophagie, satellisation, minoration, glottophobie... Ils ont aussi emprunté des concepts à des travaux convergents dans d'autres domaines, notamment en sociologie, en psychologie sociale, en sciences politiques: hégémonie, domination, marché linguistique, capital linguistique, stigmatisation, faces, aliénation, haine de soi ou « auto-odi » (Alén Garabato et Colonna, 2016), discrimination... à travers lesquels on reconnait entre autres des sources comme Gramsci (2007 [1948] via Marcellesi, 2003), Marx (via Lafont, 1965), Bourdieu (1982 et 2001), Goffman (1975), Lessing (2011 [1930], via Ninyoles, 1969)... Ces clarifications conceptuelles contribuent à identifier et à nommer des phénomènes sociolinguistiques souvent peu ou mal perçus parce que dissimulés par les idéologies dominantes, voire hégémoniques. Elles ont été largement accompagnées de propositions de transformations sociales et donc sociolinguistiques (et réciproquement), par exemple avec la proposition d'éducation linguistique démocratique de De Mauro (2018 [1974])르, de retrousser la diglossie de Lafont (1984), de normalisation (au sens de « retrouver la normalité ») chère aux sociolinguistes catalans, de Linguistic Human Rights de Skutnabb-Kangas et Phillipson (1995), de glottopolitique autogestionnaire de Guespin et Marcellesi (1986), etc.

1. Voir Escudé, Arabyan et Bronckart, 2020. 
Il s'agit là d'un projet transversal, définitoire du champ sociolinguistique avec quelques autres questions clés - toutes reliées - comme par exemple celle de la variation ou des politiques linguistiques. En effet, d'une part, la sociolinguistique est l'étude des corrélations entre langues et sociétés, c'est-à-dire des pratiques linguistiques comme pratiques sociales et des pratiques sociales sous leurs facettes linguistiques. Et, d'autre part, toute société (sauf exception non attestée à ma connaissance) est traversée par des enjeux de domination, de pouvoir, d'inégalité, d'injustice. Au croisement des deux se développe alors, de façon logique et pertinente, une étude des enjeux d'inégalités sociolinguistiques, au double sens d'inégalités linguistiques liées à des inégalités sociales et d'inégalités sociales manifestées par des inégalités de pratiques linguistiques.

Ce travail continu se poursuit aujourd'hui avec l'apparition d'approches complémentaires. Au-delà de mes propres orientations autour du concept de glottophobie récemment et largement diffusé (Blanchet, 2019 [2016]), ont été proposés : le concept de bientraitance linguistique lié à son miroir inverse la maltraitance linguistique (Dinvaut et Biichlé, 2020) et, avec le présent volume, le concept de vulnérabilité linguistique, ici soumis à l'analyse.

Les travaux ici réunis proposent le concept de vulnérabilité pour rendre compte de phénomènes affectant des langues, des communautés, des personnes. Il est explicitement corrélé à ceux d'insécurité, de glottophobie. Il permet de poser une nouvelle fois la question de la stigmatisation, notamment sous la forme de la condescendance, et des discriminations dont le volet linguistique n'est pas dissocié d'autres prétextes de stigmatisation et de discrimination, fréquemment celui de l'origine de personnes qui subissent simultanément un traitement xénophobe voire raciste, ainsi que celui de statut social. Les contributions à ce volume, grâce à leur diversité (de terrains, de contextes, de langues, etc.), permettent de le montrer de façon à la fois éclairante par ses corpus et convaincante par l'analyse cumulative de cas comparables. Maud Sérusclat-Natale le dit explicitement dans son texte sur les expériences artistiques en contexte plurilingue : "La vulnérabilité linguistique d’un individu est occasionnée par l'interaction entre une personne et son environnement au sens large » ( $p$. ABC).

C'est aussi ce que pointent Leonor Delgadillo, José Arce et Susana Pastrana dans leur analyse, chez les locuteurs de langues dites indigènes à Mexico, de cette vulnérabilité qui a:

un carácter bifacético (...) no se garantiza a los hablantes indígenas el efectivo ejercicio de sus derechos humanos, lo que les deja en condiciones de exclusión y vulnerabilidad social (...) En dicho proceso de internalización y réplica, y obediente al contexto y el momento histórico, el individuo está sometido a las premisas ideológicas sostenidas a partir de los modelos a los que puede acceder y que están vinculadas a significados comunes y colectivos, que son asumidas como verdaderas por el individuo y ante las que, de manera individual, poco o nada puede hacer (...) no solo existe la vulnerabilidad del hablante indígena sino también la vulnerabilidad de la lengua. (p. ABC) 
La corrélation entre vulnérabilité sociale et vulnérabilité linguistique met le doigt sur la question conjointe du statut social des personnes et du statut social de leur(s) langue(s) propre(s). Cette corrélation est examinée d'une façon complémentaire par Vasumathi Badrinathan dans un contexte différent, celui des enseignants indiens de français langue étrangère (FLE) en Inde : «En classe ils [les enseignants indiens de FLE] se sentent en sécurité, par rapport à la hiérarchie supérieure devant les apprenants, le pouvoir de diriger le cours et la parole » (p. ABC). Et pourtant, la situation de professeurs « non-natifs » est ambivalente (à la fois sécure et insécure) comme le montre Claudia Rincón Restrepo à propos de Colombiens:

Pour le professeur de langue étrangère (LE) non natif, cette insécurité peut nuire à son développement professionnel, voire personnel (...) La figure du locuteur «natif » représente, de manière ambivalente, un fantasme et une source d'insécurité linguistique. (...) Cette idéalisation du natif se voit parfois reflétée dans les offres de travail, car les institutions cherchent des « natifs » pour plus tard mieux vendre leurs services, renforçant ainsi l'idée stéréotypée du « natif » auprès de la population (...) Cette situation génère un énorme désavantage entre les professeurs formés dans les universités colombiennes ayant acquis des connaissances linguistiques et pédagogiques solides, et les natifs qui n'ont parfois même pas de formation en enseignement de leur LM. Le désavantage est, bien sûr, encore plus marqué si ceux-ci ont suivi une formation pédagogique et didactique en LE. Avoir un professeur «natif » est devenu un privilège dans ce pays sud-américain. Ils sont généralement mieux payés, même quand ils ont une formation plus médiocre. Dans cette échelle professionnelle, l'enseignant latino-américain subira, outre l'insécurité linguistique, une discrimination à l'emploi. (p. ABC)

On le voit : cette notion de vulnérabilité est directement liée à celle de discrimination à la fois glottophobe et sociale ainsi que d'insécurité linguistique elle aussi simultanément statutaire dans l'environnement social. Le sociologue Robert Castel (2013) distingue trois zones de la vie sociale, dont une dite de vulnérabilité intermédiaire entre inclusion et exclusion:

- une «zone d'intégration », dont font partie en général des personnes ayant un travail régulier et des supports de sociabilité suffisamment fermes;

- une «zone de vulnérabilité », regroupant les personnes ayant un travail précaire et des situations relationnelles instables;

- une «zone d'exclusion », dans laquelle certains des vulnérables, et même des intégrés basculent.

La mise en vulnérabilité apparait alors comme une phase de renvoi, une phase de transition vers l'exclusion, et probablement pas l'inverse vu sa connotation négative. 


\section{Le domaine de l'éducation : apprenants et enseignants}

Le dernier exemple cité ci-dessus attire notre attention sur le rôle de l'éducation, plus précisément sur le rôle des institutions éducatives. Dans les différents contextes investigués, on trouve une constante : l'existence d'un modèle linguistique dominant à la fois par une langue unique et par une variété unique de cette langue posée comme norme exclusive, qu'il s'agisse de l'espagnol au Mexique et en Colombie, du français en Belgique francophone et en France, et même du français standard de France enseigné comme «FLE » en Belgique, en Colombie, en Inde. La question des enfants migrants et des enseignants non natifs (d'une certaine façon « migrant entre les langues ») constitue un excellent révélateur d'un modèle aussi fantasmatique qu'abusif : un éventuel locuteur «monolingue mono normatif natif » parlant une langue «standardisée »². Par définition, ce modèle place en situation de vulnérabilité tous ceux et celles qui cherchent à entrer dans cet espace sociolinguistique (celui du français ou de l'espagnol, ici) comme locuteurs langue 2 ou 3 ou 4..., qui sont $a$ priori et irrémédiablement disqualifié.e.s. Ainsi, Maud Sérusclat-Natale constate que ces « situations de vulnérabilité rencontrées par les élèves et les enseignants [sont] souvent érigées par le système scolaire lui-même » (p. ABC). C'est, du reste, de cette façon que Francard (1993) a défini et expliqué l'insécurité linguistique : la prise de conscience d'un écart, d'une différence, entre ce que l'on parle et le modèle dominant principalement véhiculé par l'école. Même les enseignant.e.s « non natifs » sont concerné.e.s, comme le constate Vasumathi Badrinathan qui parle, dès lors, d’une :

insécurité linguistique protéiforme (...) Tous les enseignants de cette étude, sans exception, sont irréductiblement penchés vers le modèle natif. Ils ont tous évoqué la notion du « locuteur natif » comme modèle à suivre, un idéal intériorisé, qui en soi leur est valorisant. (p. ABC)

Par définition, le français est posé comme étant la langue de la France (ce qui est en grande partie faux) et tout Français ou toute Française sont posé.e.s comme «meilleur.e.s » en français que toute personne étrangère. Or, les critères de « qualité » de la langue sont discutables et, surtout, les critères de situation professionnelle n'en découlent pas automatiquement : de nombreux travaux (DerivryPlard, 2006 ; Dervin et Badrinathan, 2011) ont montré qu'un.e enseignant.e L2 ou + (L3, L4...) est mieux à même d'accompagner les personnes qui apprennent cette L2 ou + que des enseignant.e.s qui l'ont pour L1. Il s'agit bien en fait d'une double discrimination à prétexte de langue et d'origine, fondée sur des critères arbitraires, injustes, faux.

2. Faut-il encore rappeler qu'il n'existe pas de locuteur «natif » puisque personne ne parle déjà une langue ni à sa naissance ni dans les jours qui suivent, que la plupart des enfants du monde grandissent avec plusieurs langues en même temps, que la plupart des humains sont plurilingues, que toutes les langues sont pratiquées de façons variées et que personne ne parle exclusivement une seule langue d'une seule façon? 
De cette surnorme linguistique découle à l'école une mise en vulnérabilité encore plus forte des élèves, dont on ignore les capacités y compris linguistiques puisqu'on ne prend en compte que les capacités d'usage de cette surnomme. C'est ce que révèlent María Guadalupe Velasco Giles et José Federico Benítez Jaramillo :

Asimismo, las experiencias vividas en los talleres nos permitieron comprender que existen diversos modos en el que las escrituras juveniles se hacen presentes a través de múltiples formas emergentes que se contraponen con las normas de escrituras y lecturas de la escuela y en muchas ocasiones de la sociedad en general. (p. ABC)

\section{La différence perçue comme une déviance et donc comme une déficience : l'exemple de l'assimilation de l'allophonie à un handi- cap}

Le texte collectif du groupe de recherche ALIA observe que:

Le remplacement [en 2014], en Belgique francophone, de la catégorie d'élève non francophone par celle d'élève allophone semble être un calque du même remplacement opéré en France en 2012 (...) dans le contexte scolaire aussi, l'allophonie et la vulnérabilité peuvent être réelles, mais également imaginaires et abusives, et ces catégories peuvent générer des effets négatifs. (p. ABC)

En effet, l'Éducation nationale française (EN), qui utilise depuis 2012 la notion d'inclusion à l'école pour améliorer la prise en charge des élèves handicapés (physiques ou moteurs), y inclut aussi d'autres élèves posés comme «handicapés » ou « en difficulté » en raison d'autres facteurs tout à fait discutables ${ }^{3}$ :

Face aux exigences de la scolarité, qui mobilise obligatoirement la langue française, la situation de nouvel arrivant non francophone qui handicape l'élève lui crée un droit auquel l'institution répond en lui accordant des moyens de compensation. (Inspection générale de l'Éducation Nationale/Inspection générale de l'administration de l'Éducation Nationale et de la Recherche, 2009 : 128)

L'EN française regroupe ainsi l'ensemble de ces enfants sous la catégorie plus large d'« élèves à besoins éducatifs particuliers » ou «spécifiques »:

La notion de «scolarisation des élèves à besoins éducatifs spécifique » est récente. Elle recouvre une population d'élèves très diversifiée : handicaps physiques, sensoriels, mentaux; grandes difficultés d'apprentissage ou d'adaptation; enfants intellectuellement précoces ;

3. Italiques de l'auteur. 
enfants malades ; enfants en situation familiale ou sociale difficile ; mineurs en milieu carcéral ; élèves nouvellement arrivés en France ; enfants du voyage. (Ministère de l'Éducation nationale $)^{4}$

Le fait de ne pas être francophone mais locuteur d'autres langues y est considéré comme un handicap (éducatif). L'argument censément positif de ne plus définir ces élèves de façon négative («non francophones ») en choisissant le terme " allophones » est superficiel. D'une part parce que le fait de parler d'autres langues n'implique pas qu'on ne soit pas en même temps francophone (mais on voit bien que c'est le monolinguisme qui sert de repère), d'autre part parce que ces élèves restent définis de façon négative par la notion de handicap. Comme nous y invitent Maxime Alais, Silvia Lucchini, Salima El Karouni, Aphrodite Maravelaki et Élodie Oger, il convient donc « d'interroger les catégories » et « de dissocier allophonie et vulnérabilité » (p. ABC). Car ce qui conduit à mettre ces élèves en situation de vulnérabilité, c'est-à-dire d'exclusion potentielle, ce n'est pas la ou les langues qu'ils et elles parlent déjà (leur allophonie), c'est le fait d'imposer l'usage exclusif du français comme langue d'enseignement et d'apprentissage en Belgique francophone et en France. C'est ce monolinguisme hégémonique, posé comme une évidence, qui fait obstacle à leur scolarisation «normale » et qui crée le « handicap » et les rend vulnérables. Et pourtant la France et la Belgique ont ratifié la Convention internationale des droits de l'enfant qui interdit tout traitement différencié à prétexte linguistique (entre autre) et un droit à l'éducation qu'aucun prétexte linguistique ne peut restreindre. Et pourtant d'autres pays et de nombreuses recherches ont trouvé des solutions pour inclure ces enfants dans une éducation plurilingue...

\section{Conclusion : responsabilité des (psycho)linguistes, cumuls des discriminations et nécessité de transformation}

Dans les paragraphes précédents, j'ai insisté, de façon convergente avec les auteurs et autrices de ce volume, sur le rôle des institutions et des collectifs dans la mise en place de cette vulnérabilité linguistique, conséquence des stigmatisations et des discriminations que subissent des personnes et des populations dans des sociétés inégalitaires. Il me semble important de souligner la part qui revient aux chercheur.e.s qui ont trop souvent alimenté, par leurs travaux, ces représentations discriminantes des langues et de leurs usagers. Car si l'idée qu'il y ait des langues supérieures à d'autres, ou des locuteurs/locutrices en position de supériorité par rapport à d'autres, est attestée depuis des millénaires dans de nombreuses sociétés ${ }^{5}$, des spécialistes y ont leur part de responsabilité. Je ne reviendrai pas sur les grammairiens anciens qui, de l'antiquité à nos jours, ont maintenu en gros un même discours aussi prescriptif qu'erroné sur les langues en surévaluant un modèle antique unicitaire, notamment celui du latin renforcé par la puissance de l'Église catholique ou de l'arabe appuyé

4. http://www.esen.education.fr/fr/ressources-par-type/outils-pour-agir/le-film-annuel-des-personnels-de-direction/detail-d-une-fiche/?a=101\&cHash=edacb88737

5. Les anciens Grecs considéraient déjà que ceux et celles qui ne parlaient pas grec étaient des barbares au langage inarticulé. 
sur le texte coranique. Je ne reviendrai pas sur les instrumentalisations nationalistes et élitistes de certaines langues et de certaines de leurs variétés pour poser des dominations politiques, coloniales et sociales. Tout ceci est bien connu et même une synthèse en reste trop longue pour être exposée ici ${ }^{6}$. Je rappellerai juste en passant qu'il y a également des sociolinguistes et didacticien.ne.s des langues qui, tout en observant la diversité linguistique et les mécanismes de domination qui y sont à l'œuvre, préfèrent rester en deçà des transformations que cela peut conduire à proposer et ne pas remettre en question voire continuer à maintenir l'ordre sociolinguistique dominant (exemples dans Blanchet, Clerc et Rispail, 201477).

J'insisterai sur le rôle de linguistes dont certaines théories ont contribué à renforcer ces croyances au lieu de les déconstruire. Claudia Rincón Restrepo nous rappelle ici fort à propos que «la notion de locuteur "natif" est introduite par la linguistique traditionnelle structurale (....) et chomskyenne » (p. ABC). Elle vient reformuler la notion traditionnelle de langue « maternelle » sans y changer quoi que ce soit, notamment les croyances erronées qu'elle transporte, faute d'ailleurs d'investiguer leurs fondements, en continuant à croire qu'un enfant grandit de façon « normale » ou « naturelle » avec une seule langue dans une famille monolingue et que le plurilinguisme est exception. Plus profondément, ces théories linguistiques, dont on a facilement montré qu'elles ne s'appuient pas sur la réalité des pratiques linguistiques diversifiées des personnes et des sociétés ${ }^{8}$, élaborées par des intellectuels vivant dans les «élites » de sociétés dominantes à dominantes monolingues ${ }^{9}$, ont renforcé l'idée qu'il y aurait dans chaque langue une variété « correcte » qui suivrait un ensemble de règles définitoires de la langue, en confondant variété normative prescrite et variétés effectivement usitées (Blanchet, Calvet et Robillard, 2007). Plus apprécié par des psychologues que par des linguistes, la macro-théorie de Chomsky sert encore aujourd'hui de base principale et quasi unique aux études psycholinguistiques sur l'acquisition de « la » langue par les bébés. Les travaux de référence postulent souvent l'acquisition initiale d'une seule langue qui aurait une structure homogène. Les neurosciences et les approches cognitivistes étant aujourd'hui en position dominante, ce type de discours maintient dans les sociétés où ils circulent aujourd'hui largement, notamment dans les institutions éducatives telles que le ministère de l'Éducation nationale en France, l'idée d'une hiérarchisation des capacités linguistiques et, sur ce plan, des locuteurs/locutrices en fonction de leur rapport acquisitionnel à «la»langue.

6. Pour une synthèse voir Blanchet, Calvet et Robillard, 2007 ; ou Blanchet, 2019 [2016] et 2018.

7. Il est d'ailleurs significatif que la proposition très documentée et étayée de transformation sociodidactique sur laquelle se conclut cet article ait fait l'objet d'une réticence explicite de la part d'un didacticien de renom (Puren, 2018), sur la base d'un désaccord implicite profond quant aux objectifs même d'une didactique des langues et, partant, d'un malentendu général y compris sur les termes employés.

8. Chomsky dit d'ailleurs qu'il étudie la langue idéale d'un locuteur monolingue idéal dans un environnement idéalement monolingue.

9. Chomsky, fils d'enseignants, né à Philadelphie (États-Unis) et ayant aussi appris l'hébreu; Saussure, fils de savants, né à Genève (Suisse francophone) ayant étudié et travaillé Leipzig et à Paris. 
Un autre point qui me semble très important et qui est bien souligné par l'ensemble des recherches présentées ici, c'est le cumul des discriminations à l'intersection de plusieurs variables sociales et donc sociolinguistiques. Les personnes auprès desquelles a été identifiée une vulnérabilité linguistique sont toutes en situations de minoration : « indigènes » ou « autochtones » ou « immigrés » par rapport à une société coloniale et/ou à une langue internationale dominante (espagnol, français). Ce cumul des discriminations est très visible à propos des « indigènes » à Guanajuato (texte de Sophia Schnuchel et David Charles Wright). Il est explicitement énoncé par Claudia Torres Castilllo : « Parler une langue indigène au Mexique est signe de pauvreté » ( $p . A B C$ ). Ainsi une personne se retrouve stigmatisée et discriminée pour au moins trois prétextes cumulés : elle est pauvre, elle parle une autre langue que l'espagnol, elle est probablement indigène; ce qui conduit à d'autre prétextes comme probablement son apparence physique (ses traits phénotypiques, ses vêtements, ses ornements éventuels...), ses pratiques culturelles, etc.

La vulnérabilité, on l'a dit en ouverture, est une des formes que prend le processus d'exclusion, et ceci de façon circulaire : la stigmatisation conduit à la discrimination qui conduit à la vulnérabilité qui facilite et renforce les effets de la stigmatisation et ainsi de suite... Cercle vicieux, en tout cas du point de vue d'une certaine éthique et d'un certain projet de société. Les travaux ici réunis contribuent de façon stimulante à « documenter " (comme on dit aujourd'hui) et à éclairer l'analyse de ce processus, à la compléter voire à la renouveler en ajoutant le concept de vulnérabilité, à ma connaissance peu ou pas usité en sociolinguistique, à l'appareil scientifique existant. Pour tout ceci, je remercie très sincèrement les auteurs et les autrices de ce volume. 


\section{Références}

Alén Garabato, Carmen et Romain Colonna (dir.) (2016), Auto-odi : la « haine de soi » en sociolinguistique, Paris, L'Harmattan.

Blanchet, Philippe (2018), Éléments de sociolinguistique générale, Limoges, Lambert-Lucas.

Blanchet, Philippe (2019), Discriminations : combattre la glottophobie, $2^{e}$ éd., Limoges, LambertLucas. [1 ${ }^{\text {re }}$ éd., 2016.]

Blanchet, Philippe, Louis-Jean Calvet et Didier de Robillard (2007), Un siècle après le Cours de Saussure, la Linguistique en question, Paris, L'Harmattan.

Blanchet, Philippe, Stéphanie Clerc et Marielle Rispail (2014), « Réduire l'insécurité linguistique des élèves par une transposition didactique de la pluralité sociolinguistique : pour de nouvelles perspectives sociodidactiques avec l'exemple du Maghreb », ELA-Revue de didactologie des langues-cultures, n 175 (Insécurité linguistique en éducation: approches sociologiques comparées des élèves issus du Maghreb, sous la dir. de Bruno Garnier), p. 283-302.

Bourdieu, Pierre (2001), Langage et pouvoir symbolique, Paris, Seuil.

Bourdieu, Pierre (1982), Ce que parler veut dire : l'économie des échanges linguistiques, Paris, Fayard.

Castel, Robert (2013), «Cadrer l'exclusion », dans Saül Karsz (dir.), L'exclusion, définir pour en finir,

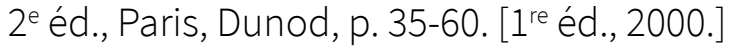

Colonna, Romain (2013), Les paradoxes de la domination linguistique : la diglossie en questions, Paris, L'Harmattan.

Derivry-Plard, Martine (2006), «Les enseignants "natifs" et "non-natifs" de langue(s) : catégorisation linguistique ou construction sociale? », Travaux de didactique du FLE, n55, p. 100-108.

Dervin, Fred et Vasumathi Badrinathan (dir.) (2011), L'enseignant non natif : identités et légitimité dans l'enseignement-apprentissage des langues étrangères, Fernelmont, Éditions Modulaires Européennes.

Dinvaut, Annemarie et Luc Biichlé (dir.) (2020), Mieux vivre en langues, ou comment passer de l'insécurité à la bienveillance, la bientraitance, la coopération, Paris, L’Harmattan.

Escudé, Pierre, Marc Arabyan et Jean-Paul Bronckart (dir.) (2020), Les langues dans la vie : hommage à Tullio De Mauro, Limoges, Lambert-Lucas.

Francard, Michel (avec la collab. de Geneviève Geron et Régine Wilmet) (dir.) (1993), L'insécuritélinguistique dans les communautés francophones périphériques : actes du colloque de Louvain-la-Neuve du 10-12 novembre 1993, Louvain-la-Neuve, Cahiers de l'Institut de Linguistique de Louvain, 2 vol.

Goffman, Erving (1975), Stigmates : les usages sociaux des handicaps, Paris, Minuit.

Guespin, Louis et Jean-Baptiste Marcellesi (1986), « Pour la glottopolitique », Langages, n 83, p. 5-34. 
Haber, Stéphane (2007), L'aliénation : vie sociale et expérience de la dépossession dans la théorie sociale, Paris, Presses universitaires de France.

Inspection générale de l'Éducation Nationale/Inspection générale de l'administration de l'Éducation Nationale et de la Recherche (2009), Rapport annuel des Inspections générales 2009, disponible sur https://www.vie-publique.fr/rapport/31337-rapport-annuel-des-inspections-generales-2009.

Lafont, Robert (1965-1967), « Sobre l'alienacion », Viure, n 1, p.6-13; n 3, p. 15-19; n 8, p. 1-9.

Lafont, Robert (1984), « Pour retrousser la diglossie », Lengas, n 15, p. 5-36.

Lessing, Theodor (2011), La haine de soi ou le refus d'être juif, Paris, Pocket. [Titre original : Der jüdische Selbsthass, Berlin, Jüdischer Verlag, 1930. Traduit de l'allemand.]

Marcellesi, Jean-Baptiste (aveclacollab. de ThierryBulotetPhilippeBlanchet) (2003), Sociolinguistique (épistémologie, langues régionales, polynomie) : textes choisis de Jean-Baptiste Marcellesi précédés d'un entretien, Paris, L'Harmattan.

De Mauro, Tullio (2018), L'educazione linguistica democratica, Roma-Bari, Laterza.

Ninyoles, Rafael Lluís (1969), Conflicte Lingüístic Valencià, Barcelona, Edicions 62.

Puren, Christian (2018), Perspective actionnelle et plurilinguisme: pour un traitement didactique et non sociolinguistique du plurilinguisme scolaire au Maghreb, disponible sur www.christianpuren. com/mes-travaux/2018g/.

Skutnabb-Kangas, Tove et Robert Phillipson (dir.) (1995), Linguistic Human Rights, Berlin/New-York, De Gruyter Mouton. 


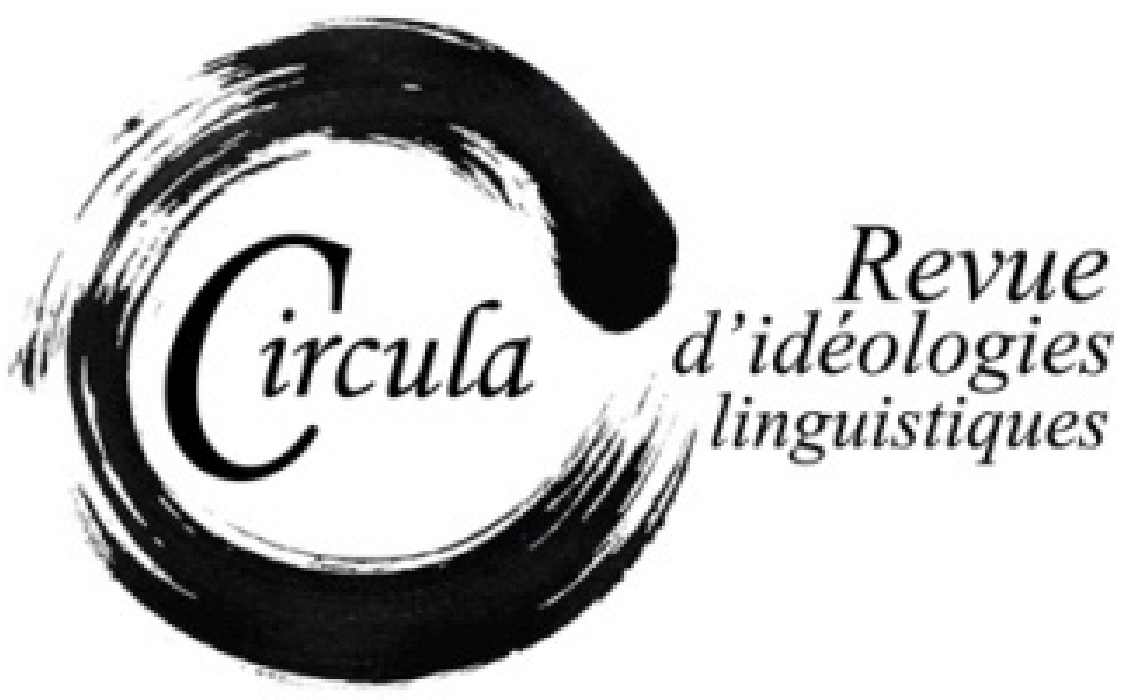

TITRE: VULNERABILIDAD DE LA LENGUA EN HABLANTES INDíGENAS, EL CASO DE MÉXICO

Auteur: Leonor Guadalupe Delgadillo GuZmán, Universidad Autónoma del ESTAdo de MÉXico, José ArCe Valdez, Universidad Autónoma del Estado de MÉxico et Susana Angélica Pastrana CORRAL, UNIVERSIDAD DE SONORA

Revue: CIRCULA, NumÉro 12 : LA VULNÉRABILITÉ LINGUISTIQUE

DiRECTRICE: CLAUdiA TORRES CASTILLO

PAGES: $18-40$

ISSN: 2369-6761

URI: HTTP://HDL.HANDLE.NET/11143/18441

DOI: HTTPS://DOI.ORG/10.17118/11143/18441 


\title{
Vulnerabilidad de la lengua en hablantes indígenas, el caso de México
}

\author{
Leonor Guadalupe Delgadillo Guzmán, Universidad Autónoma del Estado de México \\ Igdelgadillog@ uaemex.mx
}

José Arce Valdez, Universidad Autónoma del Estado de México

jarcev@uaemex.mx

Susana Angélica Pastrana Corral, Universidad de Sonora

pastrana@caborca.uson.mx

\begin{abstract}
Resumen: El actual contexto muestra una disminución de hablantes de las culturas étnicas primigenias. Se parte de datos estadísticos, marcos normativos internacionales y nacionales junto con la experiencia de hablantes indígenas, visto esto desde el modelo teórico ecológico que permite insertar el enfoque de los derechos humanos y el aprendizaje social. Se recolectó información a través de la revisión documental de escritos y audiovisuales dispuestos en Internet. El análisis se realizó de forma cualitativa a partir de la categoría de la vulnerabilidad de la lengua entendida como la fragilidad del ejercicio de la expresión verbal de la lengua materna de grupos originarios producida por el dominio circulante de otra lengua dado el reconocimiento que tiene. Se concluye la vulnerabilidad a la que es sometida la lengua.
\end{abstract}

Palabras clave: vulnerabilidad; lengua; indígenas

\begin{abstract}
The current context shows a decrease in speakers of the original ethnic cultures. This study is based on statistical data, international and national normative frameworks, together with the experience of indigenous speakers, seen from an ecological theoretical perspective that allows inserting the approach of human rights and social learning. The information gathering is done through documental and audiovisual material found on the Internet. The analysis is carried out qualitatively from the category of language vulnerability, understood as the fragility of the exercise of verbal expression of the mother language of native groups produced by the circulating dominance of another language given the recognition it has. It is conluded that language is vulnerable.
\end{abstract}

Key words: vulnerability; language; indigenous 


\section{Introducción}

Un tema relevante para la Organización de las Naciones Unidas para la Educación, la Ciencia y la Cultura (UNESCO, 2003) en su agenda de trabajo es la preservación de las lenguas y dialectos que se encuentran bajo amenaza. Ambos son sistemas de comunicación verbal, sin embargo, la distinción entre ellos estriba en los mecanismos de legitimación, es decir, de reconocimiento por parte de los gobiernos para el caso de las lenguas y, para los dialectos, su habla delimitada en cierta zona geográfica (Real Academia Española, citada por la UNESCO, 2003), que puede o no tener un sistema de escritura y cuya data es ancestral, originaria (National Geographic España, 2020). La Academia Mexicana de la Lengua (2017) indica que una lengua o idioma lo es siempre y cuando se trate de un sistema de comunicación verbal o escrita establecido de forma convenida. Mientras que un dialecto es una manera particular o local o regional de uso de la lengua. Para quienes esto escriben, los sistemas lingüísticos nativos, más allá de su categorización, encierran la forma en cómo toda una colectividad se conecta con su entorno y desarrolla un conocimiento profundo de las características y ciclos de vida del lugar en el que vive, de sus ecosistemas y demás saberes que resultan ajenos a los extranjeros.

Se dice que cada quince días una lengua muere, y a finales de este siglo XXI habrán desaparecido cerca de la mitad de las aproximadas 7,000 lenguas que actualmente existen en el mundo (UNESCO, 2003; Embriz y Zamora, 2012; National Geographic España, 2020;). Idiazabal y Pérez-Caurel (2019), mencionan que el fenómeno descrito tiene correlatos con condiciones de vulnerabilidad humana en el marco de las sociedades contemporáneas globales, pues la adopción de una segunda o tercera lengua con amplia presencia y mayor dominancia que la de origen representa un incremento significativo respecto del acceso a oportunidades de desarrollo social como la educación, las experiencias culturales internacionales, el trabajo e incluso la justicia.

En México, del total de la población durante 2018, 9.6\% (12 millones de personas) eran indígenas, ya sea por pertenecer a un hogar indígena, por autoidentificarse como uno de ellos o por hablar alguna lengua según la clasificación realizada por el Instituto Nacional de los Pueblos Indígenas [INPI $]^{1}$. De 7 millones que eran hablantes, 9.2\% no lo hacía en español, siendo mayor el porcentaje de mujeres monolingües (11.8\%) que hombres (6.5\%). En materia geográfica, 49.8\% de la población indígena y $59.1 \%$ de los hablantes residían en localidades rurales, ubicándose principalmente en las zonas de Oaxaca, Chiapas, México, Puebla y Veracruz (Robles, 2019).

Particularmente, en cuestión de educación y durante 2015, la población indígena presentaba un rezago significativo toda vez que $21.8 \%$ de ellos contaban con la primaria completa, y únicamente 9.7\% logró concluir la educación media superior, siendo mayor la diferencia con la media nacional en las regiones de Yucatán y Nayarit. En el mismo año, se registró que el 17.8\% de indígenas y 23\% de

1. Ver los Informes del Instituto Nacional de los Pueblos Indígenas https://www.gob.mx/inpi/documentos/informes-del-instituto-nacional-de-los-pueblos-indigena [Consultado el 4 de noviembre de 2020] 
hablantes de 15 años y más eran analfabetas, principalmente en el Estado de Guerrero. Respecto de la población docente, se observó que, durante el ciclo escolar 2015-2016, 9.2\% no hablaba ninguna de las lenguas maternas de la comunidad en las que enseñaban, mientras que, en primaria, el porcentaje era de 7.8\% (Robles y Pérez, 2017).

Este rezago educativo tiene un impacto significativo en materia de empleabilidad, pues aunado a la baja escolaridad, la incorporación a mercados laborales con sueldos competitivos se ve atravesado por la lengua de origen. Gallardo Gutiérrez (2020) afirma que el sistema educativo en México opera bajo un principio racista, en el que la educación indígena funge como un apéndice al currículum oficial, buscando con ello la superación de la lengua y la cultura en aras de lograr la consolidación de la identidad nacional y hacer frente a los desafíos globales.

Consistentemente con lo anterior, se observa que las personas indígenas (particularmente los padres) no transmiten su lengua materna a las nuevas generaciones con el fin de otorgarles mejores posibilidades de trabajo a futuro, aun cuando se reduzcan a empleos informales, con un carácter flexible (temporal) o a actividades como la agricultura, la venta de artesanías, la mendicidad y el servicio doméstico (Horbath, 2008) y pese a la posible desaparición de la lengua misma. Esto guarda una estrecha relación con la discriminación por origen étnico y su sostenimiento en el orden estructural, al colocar en desventaja a los hablantes de alguna lengua originaria frente a los hispanoparlantes.

En contraste con lo anterior, el Fondo para el Desarrollo de los Pueblos Indígenas de América Latina y el Caribe [FILAC] ${ }^{2}$ indica que existe interés para mantener la vigencia, difusión y uso de las lenguas indígenas a nivel internacional y garantizar a sus hablantes el acceso a los derechos de la población. En México, dentro del Plan de Desarrollo Nacional 2019-2024 (Poder Ejecutivo Nacional, 2019), se estipula la búsqueda de la igualdad en todos los sectores de la población. En materia de lenguas indígenas, han existido antecedentes por su preservación tal como la ampliación del programa Editorial con 675 títulos en 58 lenguas indígenas que beneficiaron durante 2018 a un millón 200 mil estudiantes indígenas y 66 mil docentes quienes recibieron material educativo en sus lenguas originarias y considerando al español como una segunda lengua (Secretaría de Educación Pública [SEP], 2018).

Actualmente, y como parte de las estrategias para reducir la infección por COVID-19 y mantener vigente el principio de igualdad de acceso a la educación, la SEP ha incorporado materiales de lectura y escritura, salud, ciudadanía, medio ambiente, entre otras, a la Estrategia Radiofónica para Comunidades y Pueblos Indígenas en 15 lenguas diferentes, lo que constata el interés manifiesto por la conservación de las lenguas en un país tan diverso como México³.

2. Ver https://www.filac.org/wp/comunicacion/actualidad-indigena/unesco-desarrolla-plan-de-accion-para-preservar-las-lenguas-indigenas-en-panama/. [Sitio consultado el 26 de octubre de 2020].

3. Ver https://www.eluniversal.com.mx/nacion/difunde-sep-clases-en-15-lenguas [Sitio consultado el 26 de octubre de 2020]. 
Las evidencias enlistadas anteriormente muestran un carácter bifacético, en el que, por una parte, existe una preocupación por mantener las lenguas indígenas vivas y preservar su legado cultural a través de la puesta en marcha de acciones institucionalizadas, en las que se romantiza su imagen, se exalta el folklore de sus costumbres, lo autóctono y el respeto a la identidad, con las que se cubre la cuota de acciones que, desde la mirada internacional, buscan resarcir el daño histórico a los grupos, pero por otra parte, no se establecen los mecanismos de vigilancia de cumplimiento de los marcos normativos vigentes en la materia a nivel macroestructural, por ende, no impactan en la cotidianidad del grueso de la población.

De esta forma, no se promueve integralmente su preservación bajo la legítima diversidad existente pues no se garantiza a los hablantes indígenas el efectivo ejercicio de sus derechos humanos, lo que les deja en condiciones de exclusión y vulnerabilidad social (Reyes y Bayona, 2011). De manera particular, este escenario tiene repercusiones para ellos en múltiples esferas sociales: trabajo, salud, educación, que en su conjunto impacta en su calidad de vida, a tal punto que su longevidad es inferior por aproximadamente 30 años en comparación con las personas no indígenas ${ }^{4}$.

Una de las líneas de pensamiento que permite la explicación de dicha desaparición tiene que ver con la colonialidad del lenguaje (Veronelli, 2015) y la globalización, ambos fenómenos que muestran cómo, en lo discursivo, conviven representaciones simbólicas acerca de la inferioridad/superioridad racial, del éxito y el bienestar, que son diseminadas por los diversos agentes de socialización partícipes de las industrias culturales o en el contexto más inmediato como la escuela o el trabajo, y que enfatizan cómo la apropiación de un idioma, particularmente el inglés, se asocia con la integración del sujeto a un mercado de oportunidades más amplio, que le remunera en mayores posibilidades de desarrollo (La Paz, 2012).

En este sentido, la lengua, como herramienta transmisora de la cultura, se ve invadida por una serie de premisas ideológicas macrosistémicas que se cuelan en las prácticas discursivas y que se sostienen por la repetición constante en los medios de comunicación, así como en los programas educativos o el dominio de los recursos tecnológicos, interviniendo en la forma de estructurar la realidad.

La supremacía de una lengua pone en desventaja a aquellas que se encuentran subordinadas por lo que, dentro del imaginario popular, se recrean las ideas en las que los hablantes de la lengua mayoritaria se encuentran simbólicamente por encima de aquellos que carecen de una fuerte representatividad, tanto en número, como de presencia en los medios tal como lo señala La Paz (2012).

La lengua, como ente vivo, se encuentra inserta en un ciclo infinito en el que existe en conjunto con los seres humanos, quienes la generan y reproducen, pero a quienes estructura en su identidad (Arrieta Meza, 2011). Así, el poder de la lengua conlleva al entendimiento del sujeto sobre sí mismo,

4. Ver la nota "Discriminados por hablar en su idioma natal" https://elpais.com/internacional/2014/04/16/actualidad/1397683862_926411.html [Sitio consultado el 26 de octubre de 2020]. 
sobre su capacidad de comprender la realidad a través de las palabras y representarlas a nivel de conciencia, sin embargo, cuando es transmitida de generación en generación, adquiere un carácter de independencia de la experiencia subjetiva y es aprehendida como un ente autónomo (Berger y Luckmann, 2011). Este proceso de internalización tiene lugar desde el núcleo familiar, donde la realidad signada por la lengua se aparece de forma objetiva, alejada de cualquier posibilidad interpretativa del infante, adquiriéndose la noción de las cosas, lo que son y cómo son nombradas (Arrieta Meza, 2011) y en las que se crea la propia identidad, la afiliación/rechazo a los grupos étnicos y el estigma que sobre ellos y su lengua recae.

La legitimación de tal orden lingüístico se produce desde el nivel de lo pre teórico y lo teórico colectivo o también nombrado teórico rudimentario. En dicho proceso de internalización y réplica, y obediente al contexto y el momento histórico, el individuo está sometido a las premisas ideológicas sostenidas a partir de los modelos a los que puede acceder y que están vinculadas a significados comunes y colectivos, que son asumidas como verdaderas por el individuo y ante las que, de manera individual, poco o nada puede hacer. De esta forma, el sujeto aprende y significa la realidad a través de las palabras que desde su microsistema y mesosistema le son dadas (Yáñez, 2010).

La existencia de la lengua indígena está sujeta a un estado explícito de vulnerabilidad no solo por el número reducido de hablantes sino porque en los niveles exo y macrosistémicos, en los que supuestamente podrían incidir por legítimo goce y ejercicio de sus derechos humanos, su capacidad de impacto es escasa en tanto sus hablantes no tienen poder sobre las políticas públicas y programas, o como lo indica el Instituto Nacional de las Lenguas Indígenas [INALI] (2009), resulta insuficiente la pertinencia cultural desde un enfoque intercultural que tenga efectos en toda la población del país. Así, la existencia de las lenguas se sostiene bajo un esquema necesario en términos políticos, pero carece de representación ante una estructura macrosistémica en la que el castellano se muestra en toda su hegemonía en lo económico, lo valorativo, los medios de comunicación y los marcos normativos jurídicos (Bronfenbrenner, 1979b), en otras palabras, la involuntariedad y coerción que el sistema social ejerce sobre el hablante, lo coloca como un mero receptor de todo aquello que se asume como necesario para el ejercicio básico de la vida cotidiana (y en lo que la figura de los indígenas no aparece con marcado énfasis), a la vez de convertirse en un reproductor del sistema social.

El microsistema (Bronfenbrenner, 1979b) es el nivel más próximo en el que se desenvuelven las personas, y en el que se aprende lo esencial para saber sortear las vicisitudes diarias. En este marco existe una línea franca de vulnerabilidad para la lengua, y es que, si el sujeto, hablante indígena, se encuentra en un marco en el que no accede de manera plena a los satisfactores básicos, o hay un contraste significativo entre su mundo inmediato y otros mundos de vida, ocurrirán actos en los que existe la necesidad de apropiarse de una lengua que sea más competitiva que la suya. 
Así, en el nivel del mesosistema (Bronfenbrenner, 1979a), las cosas no son lineales ni planas, se produce una relación de quiebre, pues la dinámica entre dos o más ambientes pone en disyuntiva al sujeto, quien introyectará el conocimiento de la lengua materna, pero también el castellano por resultar un medio de gestión de recursos. Situación que abre su mirada a espacios ajenos, a nuevas realidades que ponen en tensión la identidad que - hasta ese punto- tenía asumida, en las que se observa la predominancia de la lengua hegemónica y la reducida posibilidad de comunicación bajo la propia.

Al asumir que la vida está preconfigurada en castellano, las personas indígenas tienen una tarea pendiente: aprender la lengua hegemónica, misma que servirá para lograr una inserción social efectiva, esto es, en la que se goce de plenitud de derechos que no han sido producidos por su comunidad, sino que se han elaborado por personas con una visión distinta, basada en principios culturales que distan -en poca o gran medida- de los propios (Gallardo Gutierrez, 2020). Así, la idea de la identidad (que incluye y excluye elementos para afirmar la existencia de uno mismo) puede ponerse en discusión, sobre todo cuando la madurez cognitiva permite la elaboración de escenarios y acciones hipotéticas para resolver los conflictos del día a día (Castellanos, 2000). El sujeto, en este panorama, puede colocar a su lengua materna en situación de vulnerabilidad siempre que tenga la posibilidad de renunciar a ella como un elemento identitario y asumirse como un hablante de la lengua hegemónica.

De manera contraria, el reconocimiento de la lengua materna como parte de la identidad se asumirá como elemento fundamental y definitorio en el mismo punto: cuando las personas desarrollan la capacidad de autoeficacia, es decir, de ser congruentes en su hacer, pensar y sentir respecto de un objeto y realizan una valoración positiva de sus actos (Bandura, 1987).

La lengua podrá mantenerse viva siempre y cuando exista una suerte de resistencia por parte del sujeto, que se oponga a la dominación de su espacio simbólico y de sus prácticas, llevándolo a un papel que se configura como defensor de todo aquello que su lengua encierra. De acuerdo con Bandura (1987), lo aprendido está directamente relacionado con las experiencias directas e indirectas, puesto que pueden introyectarse a partir del modelo o figura que muestra cierta conducta, de las consecuencias de los actos ajenos o propios y del interés que ello despierte en el sujeto. Conforme la persona crece, su capacidad de autorregulación servirá como eje para conseguir aquellas metas que se ha propuesto (Bandura, 1987; Fuentes Vilugrón, 2020).

Este proceso, en el plano de la lengua y de los hablantes indígenas, sobre la importancia de los modelos, cercanos o ideales, es fundamental puesto que refuerzan o castigan la expresión de la lengua materna y, en virtud de ello, habilitan o frenan el sostenimiento de la lengua. Y pese a que desde los niveles exosistémico y macrosistémico se empujen políticas públicas y programas sociales de revitalización por parte de las instancias gubernamentales, éstas a su vez tienen que competir con el o los modelos que otros sistemas colocan, como las campañas publicitarias, las tecnologías, el trabajo, el transporte, la educación y la comunicación, por mencionar algunos. 
Dichos escenarios permiten develar una noción que puede denominarse "vulnerabilidad de la lengua", categoría que evidencia la fragilidad del ejercicio de la expresión verbal de la lengua materna de grupos originarios producida por el dominio circulante de otra lengua dado el reconocimiento que tiene y cuya fuerza relega a la lengua materna, al punto de someterla a un confinamiento expresivo por no ser valorada socialmente de la misma manera que la lengua dominante. Desde esta perspectiva, la condición de vulnerabilidad es otorgada por la dominancia de prácticas y discursos que sostienen a las lenguas hegemónicas en su postura de privilegio, pero, finalmente, la supervivencia de la lengua originaria se encuentra en el sujeto y el análisis que hace de su utilidad en la vida cotidiana. Ante lo propuesto cabe preguntarse ¿Cómo se manifiesta la vulnerabilidad de la lengua en los discursos de personas indígenas en México?

\section{Aspectos metodológicos}

Para dar solución a la pregunta de investigación, se realizó un estudio de corte cualitativo (Ruíz, 1996) bajo el enfoque documental. Se hizo uso de la categoría de análisis referente a la vulnerabilidad de la lengua.

Este tipo de estudios arrojan una visión panorámica actualizada sobre lo que se analiza en función de una interpretación acerca de los discursos presentes en los documentos analizados (Camarillo, 2015). La propuesta anterior se fundamentó en el análisis de documentos oficiales, así como de testimonios escritos o presentes en registros audiovisuales dispuestos en redes socio digitales sobre experiencias desfavorables de hablantes indígenas. El análisis de la información se realizó en función de un proceder interpretativo mixto (Ruíz, 1996).

\section{Resultados}

Se muestran los hallazgos de los documentos rastreados en el orden de los niveles sociales propuestos por el modelo ecológico de Bronfenbrenner (1979b): macrosistema, exosistema, mesosistema y microsistema en los que fueron encontradas evidencias del tratamiento a las lenguas indígenas.

\subsection{Macro sistema: marcos normativos nacionales}

En materia de los marcos normativos nacionales e internacionales como expresiones macrosistémicas se observó que el esfuerzo jurídico que ha realizado México para proteger tanto a los hablantes indígenas como la preservación de sus lenguas ha sido convencional con la Declaración Universal de los Derechos Lingüísticos (Comité de Seguimiento de la Declaración Universal de los Derechos Lingüísticos, 1998), pues a nivel nacional, se promulgó en el año 2003 la Ley Federal para Preveniry Eliminar la Discriminación (Cámara de Diputados del H. Congreso de la Unión, 2018), enfocada a pre- 
cisar todas las prácticas discriminatorias con base en lo señalado en el artículo $1^{\circ}$ de la Constitución Políticas de los Estados Unidos Mexicanos (Cámara de Diputados del H. Congreso de la Unión, 2019).

De acuerdo con la Comisión Nacional de Derechos Humanos (2018) a partir del año en que fue promulgada esta Ley, las 32 entidades federativas del país fueron creando sus propias leyes locales contra la discriminación, misma que se entiende como:

[...] toda distinción, exclusión, restricción o preferencia que, por acción u omisión, con intención o sin ella, no sea objetiva, racional ni proporcional y tenga por objeto o resultado obstaculizar, restringir, impedir, menoscabar o anular el reconocimiento, goce o ejercicio de los derechos humanos y libertades, cuando se base en uno o más de los siguientes motivos: el origen étnico o nacional, el color de piel, la cultura, el sexo, el género, la edad, las discapacidades, la condición social, económica, de salud o jurídica, la religión, la apariencia física, las características genéticas, la situación migratoria, el embarazo, la lengua, las opiniones, las preferencias sexuales, la identidad o filiación política, el estado civil, la situación familiar, las responsabilidades familiares, el idioma los antecedentes penales o cualquier otro motivo. [...] la homofobia, misoginia, cualquier manifestación de xenofobia, segregación racial, antisemitismo, así como la discriminación racial y otras formas conexas de intolerancia. (Comisión Nacional de Derechos Humanos, 2018: 5-6)

Junto con esta ley se promulgó en el mismo año de 2003, la Ley General de Derechos Lingüísticos de los Pueblos Indígenas, que en su artículo $2^{\circ}$ define a las lenguas indígenas como aquellas que provienen de pueblos preexistentes a la constitución del Estado mexicano, reconocidas por su capital cultural, oral y simbólico y en su tercer artículo establece que "son parte integrante del patrimonio cultural y lingüístico nacional [...] es una de las principales expresiones de la composición pluricultural de la Nación Mexicana" (Cámara de Diputados del H. Consejo de la Unión, 2018: 1).

Por su parte, el Instituto Nacional de Lenguas Indígenas, instrumentó su propio Programa institucional, conocido como PINALI (INALI, 2009). A decir de este instituto la población indígena en el país se concentra en el 6.5\% de la población, en el rango etario de 3 años y más (Embriz y Zamora, 2012). A pesar de que en el año 2003 se reconocieron como lenguas nacionales a las lenguas indígenas, sin diferenciación de validez para con el español, la disminución de hablantes originarios muestra que México carece de la capacidad como país de vivirse multilingüe. A ello se suma la exclusión de la enseñanza-aprendizaje de la lengua materna, la cultura e identidad en el nivel escolar básico (Gallardo, 2020). Según el Comité Consultivo para la Atención de las Lenguas Indígenas en riesgo de Desaparición existen cuatro diferentes grados de riesgo para que una lengua se extinga: muy alto riesgo de desaparición; alto riesgo; riesgo mediano; riesgo no inmediato (Embriz y Zamora, 2012)5.

5. Todo este marco normativo nacional mexicano revela su alineación con el objetivo mundial número 16 que forma parte de los 17 objetivos de desarrollo sostenible, trabajados de manera coordinada con el Programa de Naciones Unidas para el Desarrollo. Ver https://www.undp.org/content/undp/es/home/sustainable-development-goals.html [Sitio consultado el 22 de julio, 2020.] 
Destacan también, las acciones estructurales emprendidas a nivel internacional, ejemplo de ello es la Declaración Universal de Derechos Humanos, que desde sus artículos $1^{\circ}$ y $2^{\circ}$ enuncia la libertad e igualdad en dignidad y derechos de todas las personas sin distinción alguna de sus características personales, como el idioma (Naciones Unidas, 2015). Un segundo dispositivo internacional es la Declaración sobre los derechos de las personas pertenecientes a minorías nacionales o étnicas, religiosas y lingüísticas aprobada en 1992 (Naciones Unidas, 1992). Una tercera disposición es la Declaración Universal sobre la diversidad cultural, dictada por la Organización de las Naciones Unidas para la Educación, la Ciencia y la Cultura [UNESCO] (2001), en la que se hace hincapié que la diversidad cultural enriquece al género humano, particularmente la de los pueblos indígenas.

A lo anterior, se suma la aprobación para celebrar cada 21 de febrero el Día Internacional de la Lengua Materna, comenzándose a conmemorar en 2002. Finalmente, es importante resaltar que 2019 fue declarado "Año Internacional de las Lenguas Indígenas" con el objetivo de revitalizarlas de forma inmediata (Hetch, 2019).

\subsection{Exosistema: la vulnerabilidad de las lenguas, un problema global}

Pese a estos esfuerzos de alcance global, la discriminación en contra de las personas hablantes indígenas es un problema social que se produce igualmente a nivel global. El estudio realizado por las Naciones Unidas (2012) que abarcó a Costa Rica, Honduras, Nicaragua, El Salvador y Panamá ilustra las vivencias de cinco líderes mujeres indígenas.

Justa, originaria de Costa Rica, manifestó que su abuela materna le decía que en las escuelas les imponían muchas cosas. Ella recuerda que su maestro les decía "No hablen Bribrí, parecen animales, chanchos de monte hablando esas cosas" (Naciones Unidas, 2012: 10). Un día que la escuchó hablar en su lengua materna la exhibió enfrente de sus compañeros, poniéndola de pie y exclamando "chiquitos véanla... ¿verdad que parece salvaje?" (Naciones Unidas, 2012: 10). En el caso de Isabel, también de Costa Rica, expresa que "nosotras las mujeres somos las que más vivimos situaciones que nos afectan."

María José de Nicaragua, descendiente del pueblo indígena chorotega, narra:

Cuando egresé de la carrera de enfermería empecé a participar en las actividades de las organizaciones indígenas a nivel regional. Mi comunidad me delegó para participar en el Movimiento Indígena. Era la escribana, la persona que está detrás escuchando y escribiendo todo. No se me permitía hablar porque las asambleas eran un espacio de los hombres [...] La discriminación la percibimos en todas partes, en el trabajo, en la escuela. En los centros de salud que no nos atienden bien por ser indígenas o porque no estamos bien arreglados. La discriminación está en todas partes. (Naciones Unidas, 2012: 62). 
José Cavero de Perú, originario andino de Apurimac, residente en Lima, expone:

Desde niño aprendí que el quechua no era algo bueno. Mi mamá decía que no lo iba a usar en Lima y mi papá no me enseñó por vanidad, ya que él no aceptaba por ningún motivo que le digan serrano, y en aquel tiempo la discriminación era muy dura para los migrantes. (Casma, 2014: s.p.)

Como se observa no sólo al exterior de la misma comunidad indígena la lengua es colocada en situación de vulnerabilidad, sino también al interior con motivo de las pautas culturales y de organización social que privan, que tal y como se advierte se trata de estructuras y dinámicas patriarcales. De ello se puede confirmar lo que señala Gugenberger (1997) con base en la lengua dominante como instrumento de poder sobre la lengua o las lenguas no dominantes, que sin dificultad derivan en prácticas discriminatorias.

\subsection{Mesosistema: la vulnerabilidad de la lengua en México}

En México, los estudios realizados ligados a la violencia discriminatoria ilustran que existe todo un conjunto de prácticas desfavorables en la interacción social con motivo del "tono de piel, manera de hablar, peso, estatura, forma de vestir o arreglo personal, clase social, creencias religiosas, sexo, edad, y orientación sexual" (Instituto Nacional de Estadística y Geografía, 2018: 1). Es claro que varios de estos elementos están asociados a la caracterización general de los pueblos indígenas en el país, ser morenos, de baja estatura, con complexión robusta, con vestimenta que combina elementos propios de su pueblo originario, económicamente desaventajados. Esta combinación asoma una discriminación múltiple, a la que se suma su bilingüismo, el dominio de su lengua materna y del español.

El INALI (2009) indica que a pesar de la existencia de la Ley General de Derechos Lingüísticos de los Pueblos Indígenas prevalece la vulnerabilidad de la lengua macrosistémica y exosistémica por la falta de cumplimiento de este mandato jurídico, en el que los diferentes niveles de administración ejecutiva incurren en omisión. Lo que produce una afectación de acceso en ambientes mesosistémicos de los hablantes de lenguas, como lo son servicios de salud, educativos y de justicia.

Esto se confirma al cruzar los datos arrojados por el estudio realizado por el Consejo Nacional para la Prevención de la Discriminación, mismo en el que se señala que el 20.3\% de la población encuestada manifestó haber vivido durante los últimos doce meses, algún evento discriminatorio en su contra. Los principales ambientes en los que se perpetraron estas prácticas fueron los servicios de salud, el espacio público, el transporte y al interior de la familia. Su valor como personas es inferior al de las personas no indígenas. Situación que se agrava si sufren pobreza, o alguna discapacidad, o si su piel es oscura, en otras palabras, no es poco común que las personas indígenas hablantes vivan discriminaciones múltiples (CONAPRED, 2017). Esto estalla en la desigualdad de trato, de oportuni- 
dades y de condiciones de vida (Fuentes-Nieva, 2020). En casos extremos de violencia se pierde la libertad o hasta la vida, tal y como queda ilustrado en los siguientes cinco casos ocurridos en México ${ }^{6}$ :

- El 8 de abril del 2014, Feliciano Díaz, un niño tzotzil de 10 años vendía dulces para comprar sus útiles escolares, un funcionario del municipio de Tabasco lo humilló y lo obligó a tirar su mercancía.

- Tres tzeltales de Chiapas fueron detenidos durante nueve días [...] agentes de migración dijeron que no eran mexicanos, sino que eran originarios de Guatemala. Con dificultad hablaban y entendían el español, y aun cuando enseñaron copias de sus documentos de identidad mexicana, los agentes dijeron que eran falsos y los llevaron a un centro de retención.

- Dos mujeres otomíes de Querétaro, Alberta Alcántara y Teresa González, en 2006 fueron acusadas de manera injusta e ilógica por presuntamente haber secuestrado a seis elementos de la ahora extinta Agencia Federal de Investigación [AFI] en el estado de Querétaro. Después de tres años fueron puestas en libertad. Durante 2013 se les dictaminó reparación de daños.

- José Sánchez Carrasco, originario de la población tarahumara de Guachochi, Chihuahua, falleció en Guaymas, Sonora porque se le negó la atención médica que requería al no contar con los recursos económicos para cubrirla.

- El 16 de abril del 2014 en Guerrero, una mujer indígena embarazada falleció, al igual que su bebé de ocho meses de gestación, en la sala de espera del Hospital Básico Comunitario de Copala, Guerrero, debido a que se le negó atención ginecológica.

Los datos sociodemográficos de los pueblos indígenas en México no son nada alentadores, se reporta un rezago importante en ambos géneros, tanto en varones como en mujeres. El 36\% de mujeres hablantes indígenas con edad de 15 años o más, carecen de instrucción, y una proporción menor a la cuarta parte apenas logra finalizar la primaria. Los varones en comparación de las mujeres cuentan con mayor habilitación escolar, un 1.2 años más, sin embargo, esta ventaja sigue siendo insuficiente. No sólo se advierte esta brecha entre los sexos de los hablantes indígenas, también se encuentra la relacionada con el monolingüismo: son más las mujeres que se mantienen así en comparación con los varones.

Los datos muestran que conforme los varones van creciendo adquieren el conocimiento y dominio del español, por ser el sexo favorecido para socializar en ámbitos diversos al de su comunidad, mientras que las mujeres se encuentran sujetas a mantenerse en la vida doméstica y en consecuencia al interior de la comunidad. Esta condición favorece que la lengua se feminice (Instituto Nacional de las Mujeres, 2005).

6. Ver la nota del portal Redacción Wipy del 9 de agosto de 2015 "5 casos de discriminación a indígenas en México" https://wipy.tv/5-casos-discriminacion-indigenas-mexico/ [Sitio consultado el 17 de julio, 2020.] 
Las lenguas indígenas en México se encuentran en franco riesgo debido a la discriminación a pesar de que sea uno de los países a nivel mundial con una de las mayores riquezas culturales y lingüísticas. Esta acción de repudio favorece que el hablante indígena opte por no expresarse en su propio idioma y hacerlo a través del castellano (Horbath, 2008).

Hernández-Rosete y Maya (2015) coinciden que la discriminación sobre una lengua es una muestra más del poder opresivo, el uso malicioso de las relaciones sociales desiguales reforzadas por el peso de la historia y la tolerancia social.

\subsection{Microsistema: la renuncia individual a la lengua}

Los ocho casos que a continuación se presentan revelan las prácticas discriminatorias de las que son víctimas las personas indígenas en México. La tabla siguiente da cuenta de los casos recuperados, su sexo, origen, los derechos transgredidos y los sistemas ambientales activados (ver tabla 1).

\begin{tabular}{|c|c|c|c|c|}
\hline Caso & Sexo & Origen & $\begin{array}{l}\text { Derechos } \\
\text { transgredidos }\end{array}$ & $\begin{array}{c}\text { Sistemas } \\
\text { involucrados }\end{array}$ \\
\hline Yásnaya Aguilar & Femenino & Mixe & $\begin{array}{c}\text { Dignidad } \\
\text { Patrimonial } \\
\text { Vida }\end{array}$ & $\begin{array}{c}\text { Mesosistema } \\
\text { Exosistema }\end{array}$ \\
\hline $\begin{array}{c}\text { Mardonio Carba- } \\
\text { llo }\end{array}$ & Masculino & Nahuatlaco & $\begin{array}{c}\text { Dignidad } \\
\text { Integridad }\end{array}$ & Mesosistema \\
\hline Oaxaqueña & Femenino & Zapoteca & $\begin{array}{c}\text { Dignidad } \\
\text { Patrimonio }\end{array}$ & Mesosistema \\
\hline Juanita & Femenino & Raramuri & $\begin{array}{l}\text { Dignidad } \\
\text { Integridad }\end{array}$ & $\begin{array}{c}\text { Mesosistema } \\
\text { Exosistema }\end{array}$ \\
\hline Víctor & Masculino & $\begin{array}{c}\text { Tzotzil } \\
\text { chamula }\end{array}$ & Dignidad & Mesosistema \\
\hline Santos & Masculino & Tzotzil & Dignidad & Mesosistema \\
\hline Manuel & Masculino & Tzotzil & Dignidad & $\begin{array}{c}\text { Mesosistema } \\
\text { Exosistema }\end{array}$ \\
\hline
\end{tabular}




\begin{tabular}{|c|c|c|c|c|}
\hline Caso & Sexo & Origen & $\begin{array}{c}\text { Derechos } \\
\text { transgredidos }\end{array}$ & $\begin{array}{c}\text { Sistemas } \\
\text { involucrados }\end{array}$ \\
\hline Andrea & Femenino & No se precisa & Integridad & Mesosistema \\
& & & Patrimonio & Exosistema \\
\hline
\end{tabular}

Tabla 1. Análisis de la transgresión a derechos humanos en personas indígenas por efecto de la lengua

Yásnaya Aguilar, lingüista y activista mixe de la región de Oaxaca, México expone:

¿[... de pronto decidimos abandonar nuestras lenguas? No fue así. Se trató de un proceso impulsado desde las políticas del gobierno y se les quitó el valor en favor de una lengua única: el español. Para lograr la desaparición de nuestras lenguas, nuestros antepasados recibieron golpes, regaños y discriminación por el hecho de hablar sus lenguas maternas. 'Tu lengua no vale, 'Para ser ciudadano mexicano necesitas hablar la lengua nacional, el español. Deja de usar tu lengua', Los esfuerzos realizados desde el Estado fueron arduos para establecer una castellanización forzada con el fin de erradicar nuestras lenguas, sobre todo desde el sistema escolar. [...] Aun cuando han cambiado las leyes [...] continúan siendo discriminadas dentro de los sistemas educativo, de salud y del judicial. Nuestras lenguas no se mueren, las matan. A nuestras lenguas también las matan cuando no se respetan nuestros territorios, cuando las venden y concesionan, cuando asesinan a quienes las defienden. ¿Cómo vamos a florecer nuestras lenguas cuando matan a quienes las hablan, los silencias o desaparecen? [... . Nuestras lenguas no mueren, las matan. El Estado mexicano las ha borrado. El pensamiento único, la cultura única, el Estado único, con el agua de su nombre, las borra.

En este mismo sentido, Almaraz ${ }^{8}$ comenta:

A mí todavía me dieron golpes en la mano por hablar mi lengua en la escuela, pero cuando trabajé en el Instituto Nacional de Lenguas Indígenas seguí varios casos: en 2006 se reportó a una niña que fue colgada boca abajo como castigo por hablar náhuatl en clase; en 2005 una niña otomí no pudo ser registrada con su nombre en su propia lengua porque no lo permitió el registro civil; en 2015 alumnos de una secundaria eran castigados con ir a lavar los baños sí hablaban chantino en la escuela.

7. Ver "El agua y la palabra. México y sus muchos nombres ocultos" en la Revista de la Universidad de México. Disponible en https://www.revistadelauniversidad.mx/articles/a52f9f39-15d2-4993-a8a6-d8689d7995e1/el-agua-y-la-palabra [Consultado el 4 de noviembre de 2020]

8. Ver el artículo Así desaparecen las lenguas indígenas en México: "Me daban golpes en la mano por no hablar castellano en la escuela" https://www.eldiario.es/desalambre/indignacion-Mexico-Racismo-aprender-indigena_0_802120482. html [Sitio consultado el 11 de julio 2020]. 
Por su parte, Mardonio Carballo, indígena nahuatlaco de 44 años, cuenta: "Mi choque no fue tanto, pero sí fue muy violento. Mi padre se empeñó con que en casa habláramos castellano pues venía de un proceso donde a base de palos lo despojaron de su lengua9." En este testimonio puede apreciarse como, en un ejercicio de repetición institucionalizada y haciendo gala de actos violentos, el sujeto hablante indígena es despojado de su lengua para someterse a la dominante, lo que da cuenta de una realidad por demás apremiante en el sentido de la supervivencia lingüística y que muestra la insuficiencia del aparato institucional para hacer valer el respeto a las lenguas indígenas en el plano de la igualdad sustantiva, obligando a los hablantes a extinguirla paulatinamente por efecto de un condicionamiento aversivo.

El testimonio de una mujer zapoteca residente en Oaxaca, entrevistada por una cadena televisiva mexicana, muestra como el mismo condicionamiento se realiza desde una perspectiva economicista10: "En cuarto año a mí me hacían pagar cinco centavos por cada palabra que yo dijese en zapoteco, con la idea de obligarme a aprender el español y olvidar el zapoteco [...] los prejuicios lingüísticos permanentemente han existido".

Este condicionamiento que pareciera ser meramente familiar encuentra resonancia en los actores representantes del Estado. El testimonio de una mujer tarahumara, quien vive en el asentamiento raramuri Oasis en Chihuahua desde hace 28 años y que se caracteriza por un alto nivel de marginación, da cuenta de cómo la falta de traductores pone en riesgo la vida de la comunidad tarahumara, pues los servicios de salud se encuentran exclusivamente en castellano, forzando a la comunidad a ajustarse a ello:

Porque por no saber hablar uno, no nos atienden o dicen "al cabo que las tarahumaras aguantan mucho", [...] a mi si me ha ido bien. Si se siente uno mal cuando nos trata mal. En relación con los derechos de libertad refiere: "pues lo detienen, cuando uno no sabe ni por qué lo meten a la cárcel”11

Víctor, miembro perteneciente al pueblo tzotzil, ubicado en San Juan Chamula, Chiapas y maestro activo de educación básica en los altos de esta región de extrema pobreza, expresa:

Fui también víctima de la discriminación por hablar una lengua, por el simple hecho de pertenecer a un pueblo originario. Yo creo que una de las partes más crudas es ser indígena [...] la situación más difícil quizá la tienen las mujeres, porque ser mujer indígena es un factor grave para la superación académica [...] cuando te ven entrar a una tienda supuestamente de pres-

9. Ver el artículo Lingüicidio: SOS por las lenguas indígenas en la edición digital de la revista Contenido https://issuu. com/guiatkm/docs/contenido_664 [Consultado el 4 de noviembre de 2020]

10. Ver la entrevista en el reportaje audiovisual "Lengua indígena" https://www.facebook.com/watch/?v=1179644625542675 [Sitio consultado el 4 de noviembre de 2020]

11. Ver el reportaje "Discriminación indígena" https://www.facebook.com/watch/?v=1057650141099549 [Sitio consultado el 4 de noviembre de 2020]. 
tigio, exclusiva, la gente te está observando, llegas con la intención de querer esparcirte, [...] la gente te observa ¿Qué observan? La manera en que vistes, la manera en que uno habla [...] cuando uno viste el traje regional, una cosa es que lo vista un funcionario de alto nivel y otra que lo vistamos nosotros que somos dueños, del cual nos sentimos muy dignos en vestir ${ }^{12}$

En el mismo sentido, Santos, indígena Tzotzil originario de los altos de Chiapas, con conocimiento básico del castellano señala, "A causa de no hablar español, ni entender el español, la gente mestiza a veces se molesta con nosotros y por no tener estudios vienen los regaños y vienen los maltratos ${ }^{13}$ " discurso que se refuerza con lo expresado por Manuel, indígena de la misma región de México que declara: "Si nos han maltratado, salimos a buscar trabajo y no nos entregan el trabajo fácilmente, porque algunos no sabemos hablar bien el español y no es igual que los mestizos que están ahí en la ciudad que les dan trabajo"14.

Lo anterior se compagina con lo expuesto por Andrea González, mujer joven indígena, oficial de formación del Instituto de liderazgo Simone de Beauvoir, quien expone que ${ }^{15}$.

En México, el hecho de hablar una lengua indígena aún te coloca en situación de exclusión y con menores oportunidades pues para acceder a ciertos recursos como son, la educación, la salud o incluso para acceder a la justicia ¿Por qué? Porque en México se sigue privilegiando la lengua hegemónica que en este caso es el español en detrimento de las muchas lenguas indígenas que estamos en el país. Y si se supone que México debería ser un país pluricultural o multicultural pues debería garantizar también que todos los derechos de todas las personas que habitamos en este país pueda ejercerse [...] con esa discriminación tú no solo estás tratando mal a esa persona sino le estás negando sus derechos para acceder en este caso a una vivienda, para acceder a la salud, para acceder a la justicia y que entonces no estamos construyendo un país ni democrático ni en donde todos podemos convivir en equidad y en igualdad de condiciones.

Estos testimonios muestran cómo se produce una dinámica de tensión entre las personas hablantes indígenas y las no indígenas en el ambiente mesosistémico (INEGI, 2018), a través de verbalizaciones de descalificación y menosprecio como "Tu lengua no vale". El uso de actos violentos como los golpes, como la oposición de registrar el nombre propio en la lengua originaria, tratos vejatorios de

12. Ver el reportaje audiovisual "\#yabasta con la discriminación indígena, todos somos iguales. https://www.facebook. com/watch/?v=865192123894122 [Sitio consultado el 4 de noviembre de 2020].

13. Ver el reportaje audiovisual "\#yabasta con la discriminación indígena, todos somos iguales. https://www.facebook. com/watch/?v=865192123894122 [Sitio consultado el 4 de noviembre de 2020].

14. Ver el reportaje audiovisual "\#yabasta con la discriminación indígena, todos somos iguales. https://www.facebook. com/watch/?v=865192123894122 [Sitio consultado el 4 de noviembre de 2020].

15. Ver el audiovisual del Consejo Nacional para Prevenir la Discriminación "¿Cómo viven la discriminación quienes hablan una lengua indigena?" https://www.facebook.com/watch/?v=249685465935761 [Sitio consultado el 4 de noviembre de 2020] 
amenaza por parte de maestros sobre alumnos hablantes indígenas, lavar los excusados si hablaban en su lengua (Gugenberger, 1997).

La sola expresión "a base de palos lo despojaron de su lengua” no solo muestra la vulnerabilidad del hablante indígena como persona sino también la vulnerabilidad de la lengua que progresivamente se aniquila al hacer lo mismo a su hablante: medio y agente de su existencia (Bandura, 1987; Fuentes Vilugrón, 2020). El sólo hecho de mantenerse monolingüe de su propia lengua, igualmente coloca tanto al indígena hablante como a la lengua en vulnerabilidad, como tal fue el caso de Juanita, mujer rarámuri, necesitada de servicios de salud. Todo esto acontece en el nivel mesosistémico (CONAPRED, 2017).

Pero la vulnerabilidad de la lengua no se queda ahí, acontece también en el ambiente exosistémico frente a situaciones de detención y por lo tanto pérdida de la libertad, sin claridad o señalamiento explícito de la comisión del supuesto delito cometido. Situaciones en las que figuras de autoridad desconocen la documentación oficial que respalda y acredita la identidad de pertenencia nacional del hablante indígena (CONAPRED, 2017; Fuentes-Nieva, 2020). Ni que decir del acceso a oportunidades de trabajo, que no son otorgadas a este sector de la población en México porque no hablan "bien” el español.

Con ello se advierte cómo persiste una forma de postcolonialidad de la lengua en el marco del siglo XXI (Veronelli, 2015), misma que coloca un estigma a los hablantes de a las lenguas indígenas, restándoles valor y colocándoles como inferiores a aquellos que se expresan oralmente en castellano, lo que alimenta una franca contradicción entre el nivel macrosistémico y el exosistémico (Bronfenbrenner, 1979a), aquel con la promulgación de marcos normativos nacionales e internacionales en favor de la preservación y reconocimiento de las lenguas como un recurso cultural invaluable (Naciones Unidas, 2015; Cámara de Diputados del H. Congreso de la Unión, 2018; 2019), y el segundo, con la ausencia de políticas públicas contundentes que operen tal marcos normativos y con servidores públicos que se mueven en ambientes medulares de acceso al ejercicio de derechos humanos, derecho al trabajo, derecho al respeto de la identidad, derecho a servicios de salud (INALI, 2009).

Es contra todos estos elementos adversos que se conjugan en distintos ambientes con los que los hablantes indígenas tienen que lidiar, y cuyo peso aumenta si se trata de mujeres indígenas, quienes no solamente ven vulnerada su integridad y su lengua fuera de su comunidad, en un plano paralelo y simultáneo también son vulneradas como personas al interior de su grupo, "ser mujer indígena es un factor grave para la superación". Es así como los modelos "ideales” de expresión de la lengua para los hablantes indígenas resultan ajenos, impersonales, extraños y en lo cotidiano son reproducidos por no indígenas con una fuerte dosis de desprecio combinada con una curiosidad morbosa de lo autóctono en proceso de extinción (Naciones Unidas, 2012). 


\section{Conclusiones}

Se concluye que no solo existe la vulnerabilidad del hablante indígena sino también la vulnerabilidad de la lengua. La relación entre el hablante indígena y su lengua es íntima e irremplazable, que encuentra respaldo en los marcos normativos convencionales y constitucionales de México, pero no lo mismo en las situaciones cotidianas en las que hablar de manera diferente al castellano supone una barrera para el acceso a los derechos fundamentales como la salud, el trabajo o la educación.

Lo mismo sucede cuando el hablante indígena se encuentra con un discurso dual entre las múltiples instituciones socializadoras, puesto que en el plano de la convivencia cotidiana le minimiza, le oprime y le discrimina por múltiples factores entre los que se encuentra su propia forma de hablar, pero en aquellas en las que se ensalza la diversidad cultural, se muestra socialmente valorado, principalmente en los discursos de orden político y académico, que lo mantienen en la vitrina, como un objeto al que hay que respetar y admirar.

Lo que contrasta con el hecho de que a pesar de la existencia de la Ley General de Derechos Lingüísticos de los Pueblos Indígenas prevalece la vulnerabilidad de la lengua macrosistémica y exosistémica por la falta de cumplimiento de este mandato jurídico, en el que los diferentes niveles de administración ejecutiva incurren en omisión. Lo que produce una afectación de acceso en ambientes mesosistémicos de los hablantes de lenguas, como lo son servicios de salud, educativos y de justicia.

Así, en el plano de lo individual, los hablantes de las lenguas indígenas se encuentran ante una disyuntiva franca, en la que sostener sus formas de expresión lingüística conlleva un estigma que pone en riesgo la supervivencia de la lengua puesto que, al no encontrar un marco amplio de oportunidades en el que su desarrollo social y su lengua converjan, las probabilidades de mantenerla son menores.

El reto es capitalizar y fortalecer la capacidad de los indígenas hablantes en sus diferentes generaciones para lograr una participación lingüística activa desde su lengua materna, lo que bien se puede imbuir con la propagación de modelos a imitar que muestren las ventajas del bilingüismo, como la pertenencia a la comunidad a la que se pertenece, la auto-reflexión de los elementos identitarios adicionales a la lengua, como la comida, la vestimenta, las ceremonias, el conocimiento ecológico del ambiente natural de la comunidad.

Resulta urgente establecer como meta la preservación de la lengua como una evidencia plena e irrefutable de efectos cognitivos y emocionales positivos, y del reconocimiento por parte del Estado de su obligación instrumental, es decir, operativa de proteger patrimonios inmateriales característicos del país que redundan en su riqueza cultural. 
A nivel microsistémico es necesario enfatizar que el mantenimiento y sostenimiento de la lengua puede inducirse desde los mismos miembros de la comunidad, al consensuar decisiones colectivas sobre la preservación y enseñanza de la lengua original, como, por ejemplo, integrar un diccionario. Acción que requiere del respaldo objetivo, esto es, material y operativo para que institucionalmente el Estado financie y divulgue este tipo de elementos educativos articulándose con las instituciones de educación a nivel federal y estatal según región y lengua.

Un niño o adolescente tomará como referentes de la lengua a los adultos que están a su alrededor, en su microsistema y mesosistema, al ver que la comunicación está mediada por su lengua materna, estará motivado para aprenderla y producirla, lo que repercutirá en su autorregulación. Una persona que conoce y domina su capacidad autorregulatoria, planea las acciones que debe de llevar a cabo para lograr sus metas, toma las decisiones necesarias al respecto, con tal de conseguir ese objetivo que lo fortalece como ser humano y miembro de una comunidad, en este caso, una comunidad originaria ¡Hasta que la dignidad se haga costumbre! 


\section{Referencias bibliográficas}

Academia Mexicana de la Lengua (2017), Idioma y dialecto ¿Cuál es la diferencia entre un idioma y un dialecto? ¿El náhuatl es un dialecto?, disponible en http://www.academia.org.mx/espin/ respuestas/item/idioma-y-dialecto\#: :text=\%C2\%BFEl\%20n\%C3\%A1huatl\%20es\%20un\%20 dialecto,se\%20llaman\%20idiomas\%20o\%20lenguas.\&text=Con\%20respecto\%20al\%20n\%C3\%A1huatl\%2C\%20lengua,hay\%20quienes\%20lo\%20llaman\%20dialecto. [Sitio consultado el 26 de octubre de 2020.]

Arrieta Meza, Luis (2011), "Papel del lenguaje, y en particular de la lengua, en el desarrollo del género humano y en el desarrollo de la ciencia: reflexión e investigación”, Revista editorial del Congreso por una Educación de Calidad, n4, p. 236-241.

Bandura, Albert (1987), "Mecanismos autorreguladores", en Albert Bandura, Pensamiento y acción: Fundamentos sociales, Barcelona, Ediciones Martínez Roca.

Berger, Peter y Thomas Luckmann (2011), La construcción social de la realidad, Buenos Aires, Amorrortu.

Bronfenbrenner, Urie (1979a), The ecology of human development: experiments by nature and design. Estados Unidos, Harvard University Press.

Bronfenbrenner, Urie (1979b), "Contexts of Child Rearing Problems and Prospects", American Psychologist, vol. 34, n 10, p. 844-850.

Cámara de Diputados del H. Congreso de la Unión (2018), Ley Federal para Prevenir y Eliminar la Discriminación, disponible en http://www.diputados.gob.mx/LeyesBiblio/pdf/262_210618. pdf. [Sitio consultado el 14 de julio, 2020.]

Cámara de Diputados del H. Congreso de la Unión (2018), Ley General de Derechos Lingüísticos de los Pueblos Indígenas, disponible en http://www.diputados.gob.mx/LeyesBiblio/pdf/257_200618. pdf. [Sitio consultado el 12 de julio, 2020.]

Cámara de Diputados del H. Congreso de la Unión (2019), Constitución Política de los Estados Unidos Mexicanos, disponible en http://www.diputados.gob.mx/LeyesBiblio/pdf_mov/Constitucion_ Politica.pdf. [Sitio consultado el 13 de julio, 2020.]

Camarillo, María (2015), "La investigación hemerográfica”, Nueva Época, vol. XX, nos 1-2, p. 333-357, disponible en http://publicaciones.iib.unam.mx/publicaciones/index.php/boletin/article/ view/789/760. [Sitio consultado el 14 de julio, 2020.]

Casma, Julio César (2014), "Discriminados por hablar su idioma natal”, El País, disponible en https:// elpais.com/internacional/2014/04/16/actualidad/1397683862_926411.html. [Sitio consultado el 26 de octubre de 2020].

Castellanos, Alicia (2000), “Antropología y racismo en México”, Desacatos, n. 4, p.53-79. 
Comisión Nacional de Derechos Humanos [CNDH] (2018), El derecho a la no discriminación, disponible en https://www.cndh.org.mx/sites/all/doc/cartillas/2015-2016/21-Discriminacion-DH.pdf. [Sitio consultado el 4 de noviembre de 2020.]

Comité de Seguimiento de la Declaración Universal de los Derechos Lingüísticos (1998), Declaración Universal de Derechos Lingüísticos, disponible en https://www.pencatala.cat/wp-content/ uploads/2016/02/dlr_espanyol.pdf. [Sitio consultado el 26 de octubre de 2020.]

Consejo Nacional para la Prevención de la Discriminación [CONAPRED] (2017), Encuesta Nacional sobre Discriminación, disponible en http://internet.contenidos.inegi.org.mx/contenidos/ Productos/prod_serv/contenidos/espanol/bvinegi/productos/nueva_estruc/702825100711. pdf\#: :text=La\%20Encuesta\%20Nacional\%20sobre\%20Discriminaci\%C3\%B3n\%20 \%28ENADIS\%29\%202017\%20es, demogr\%C3\%A1ficos\%2C\%20econ\%C3\%B3micos\%20 y\%20socioculturales\%20que\%20se\%20le\%20relacionan. [Sitio consultado el 15 de julio, 2020.]

Embriz, Arnulfo y Óscar Zamora (2012), Lenguas Indígenas nacionales en riesgo de desaparición, México, Instituto Nacional de Lenguas Indígenas.

Fuentes Vilugrón, Gerardo Andrés (2020), "El espacio como elemento clave para la regulación emocional en la escuela: análisis en contextos de diversidad social y cultural", Revista Educación, n 44, vol. 22, disponible en https://doi.org/10.15517/REVEDU.V44I2.39365. [Consultado el 4 de noviembre de 2020.]

Fuentes-Nieva, Ricardo (2020), "México y el coronavirus: pasividad gubernamental en una sociedad desigual.", Análisis Carolina, disponible en https://doi.org/10.33960/AC_16.2020. [Sitio consultado el 14 de julio, 2020.]

Gallardo Gutiérrez, Ana Laura. (2020), "Educación indígena en tiempos de COVID-19:viejos problemas, nuevos problemas", en Hugo Casanova Cardiel (ed.), Educación y pandemia: una visión académica, (p. 164-169), Ciudad de México, Universidad Autónoma de México, Instituto de Investigaciones sobre la Universidad y la Educación.

Gugenberger, Eva (1997), “Incomunicación' y discriminación lingüística en el contexto intercultural (Perú)", en Christine Bierbach y Klaus Zimmermann (eds.), Lenguaje y comunicación intercultural en el mundo hispánico, Vervuert, Iberoamericana, p. 131-146.

Hernández-Rosete, Daniel y Olivia Maya (2015), "Discriminación lingüística y contracultura escolar indígena en la Cd. De México". Revista Latinoamericana de Ciencias Sociales, Niñez y Juventud, vol. 14, n², p. 1161-1176.

Hetch, Ana Carolina (2019), "Reflexiones sobre la diversidad lingüística en el marco del año internacional de las lenguas indígenas." Cuadernos del Instituto Nacional de Antropología y Pensamiento Latinoamericano, vol. 28, no 2, p. 138-140.

Horbath, Jorge Enrique (2008), "La discriminación laboral de los indígenas en los mercados urbanos de México: revisión y balance de un fenómeno persistente", en María del Carmen Zabala Argüelles (ed.), Pobreza, exclusión social y discriminación étnico-racial en América Latina y el Caribe, Bogotá, Siglo del hombre editores-CLACSO, p.25-52. 
Idiazabal, Itziar y Manel Pérez-Caurel, (2019), Linguistic diversity, minority languages and sustainable development. España, Servicio Editorial de la Universidad del País Vasco.

Instituto Nacional de Estadística y Geografía. (2018). Una de cada 5 personas de 18 años y más declaró haber sido discriminada en el último año: encuesta nacional sobre discriminación (ENADIS) 2017, disponible en https://www.inegi.org.mx/contenidos/saladeprensa/boletines/2018/estsociodemo/enadis2017_08.pdf. [Sitio consultado el 19 de julio de 2020.]

Instituto Nacional de las Mujeres (2005), La población indígena mexicana, disponible en http://cedoc. inmujeres.gob.mx/documentos_download/100782.pdf. [Sitio consultado el 16 de julio, 2020.]

Instituto Nacional de Lenguas Indígenas [INALI] (2009), Programa de revitalización, fortalecimiento y desarrollo de las lenguas indígenas nacionales 2008-2012, disponible en www.inali.gob.mx>pdf>PINALI-2008-2012. [Sitio consultado el 13 de julio, 2020.]

La Paz, Esteban (2012), "Imperialismo lingüístico: el caso del inglés en la educación secundaria uruguaya (1941-2003)", Lingüística, vol. 27, p. 168-196.

Naciones Unidas (1992), Declaración sobre los derechos de las personas pertenecientes a minorías nacionales o étnicas, religiosas y lingüísticas, disponible en https://www.ohchr.org/SP/ Professionallnterest/Pages/Minorities.aspx. [Sitio consultado el 14 de julio, 2020.]

Naciones Unidas (2012), Mujeres indígenas en defensa de los derechos humanos. Testimonios disponible en http://oacnudh.org/wp-content/uploads/2013/10/mujeres_indigenas_derechoshumanos.pdf. [Sitio consultado el 20 de julio, 2020.]

Naciones Unidas (2015), Declaración Universal de Derechos Humanos, disponible en https://www. un.org/es/documents/udhr/UDHR_booklet_SP_web.pdf. [Sitio consultado el 12 de julio, 2020.]

National Geographic España (2020), Lenguas en peligro de extinción, disponible en https://www.nationalgeographic.com.es/mundo-ng/grandes-reportajes/lenguas-peligro-extincion_6174/4. [Sitio consultado el 26 de octubre de 2020.]

Organización de las Naciones Unidas para la Educación, la Ciencia y la Cultura. (2001). Declaración universal de la UNESCO sobre la diversidad cultural, disponible en http://portal.unesco.org/es/ ev.php-URL_ID=13179\&URL_DO=DO_TOPIC\&URL_SECTION=201.html. [Sitio consultado el 12 de julio, 2020.]

Organización de las Naciones Unidas para la Educación, la Ciencia y la Cultura [UNESCO] (2003), Vitalidad y peligro de desaparición de las lenguas, disponible en http://www.unesco.org/new/ fileadmin/MULTIMEDIA/HQ/CLT/pdf/LVE_Spanish_EDITED\%20FOR\%20PUBLICATION.pdf. [Sitio consultado el 26 de octubre de 2020].

Poder Ejecutivo Nacional (2019), Plan de Desarrollo Nacional 2019-2024, disponible en https://www. dof.gob.mx/nota_detalle.php?codigo=5565599\&fecha=12/07/2019. [Sitio consultado el 26 de octubre de 2020.] 
Reyes, María Emma y Claudia Bayona, (2011), "Desarrollo del lenguaje y vulnerabilidad: ¿Prejuicio o fundamento científico?", Revista colombiana de medicina física y rehabilitación, vol. 10, p. 6-15, disponible en https://revistas.ecr.edu.co/index.php/RCR/article/view/72. [Sitio consultado el 26 de octubre de 2020.]

Robles Vásquez, Héctor V. y Mónica Gladis Pérez Miranda (eds.) (2017), Breve panorama educativo de la población indígena: día Internacional de los Pueblos Indígenas, México, Instituto Nacional para la Evaluación de la Educación, disponible en https://historico.mejoredu.gob.mx/wp-content/ uploads/2018/12/P3B107.pdf. [Consultado el 4 de noviembre de 2020.]

Robles Vásquez, Héctor V. (2019), Panorama educativo estatal de la población indígena 2018, México, Instituto Nacional para la Evaluación de la Educación-UNICEF, disponible en https://www.inee. edu.mx/wp-content/uploads/2019/08/P3B112.pdf.

Ruíz, José (1996), Metodología de la investigación cualitativa, Universidad de Deusto, España.

Secretaría de Educación Pública [SEP]. (2018). Comunicado 272 : amplía SEP programa editorial en 58 lenguas indigenas, disponible en https://www.gob.mx/sep/prensa/comunicado-272-amplia-sep-programa-editorial-en-58-lenguas-indigenas [Sitio consultado el 26 de octubre de 2020.]

Veronelli, Gabriela Alejandra (2015), "Sobre la colonialidad del lenguaje”, Universitas humanística, n० 81 , p. 33-58.

Yáñez Henríquez, Raúl (2010), "La construcción social de la realidad”, Ars Boni et Aequi, vol. 6, nº 2, p. 280-304. 


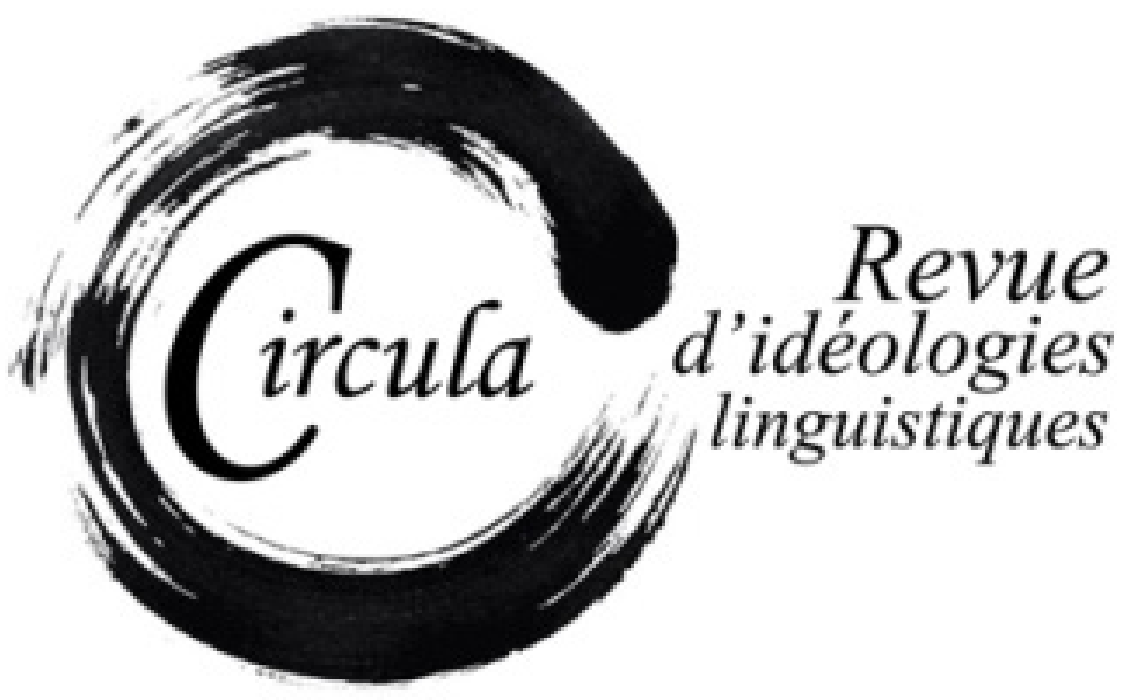

Titre: HiÉRARCHIES IMAgINÉES DES LOCUTEURS ET DES LANGUES-CULTURES AU MEXIQUE Auteur: Claudia Torres Castillo, Universidad Autónoma del Estado de México

ReVue: Circula, NUMÉRO 12 : LA VULNÉRABILITÉ LINGUISTIQUE

DiRECTRICE: Claudia TORRES CASTILLO

PAGES: $41-64$

ISSN: 2369-6761

URI: HTTP://HDL.HANDLE.NET/11143/18442

DOI: HTTPS://DOI.ORG/10.17118/11143/18442 


\title{
Hiérarchies imaginées des locuteurs et des langues-cultures au Mexique
}

\author{
Claudia Torres Castillo, Universidad Autónoma del Estado de México
}

claudiatorresc@yahoo.com.mx

\begin{abstract}
Résumé : L'image ou les images que l'on peut se faire d'une langue et de ses locuteurs sont le résultat d'un processus de construction à travers différentes circonstances historiques, sociales et culturelles. En effet, les images des langues se construisent tout d'abord chez l'individu; le discours informel personnel est une manière de s'approprier la langue. Ces images des langues, constituées en imaginaires, ont été intériorisées par les membres de la société à travers le temps et par divers moyens ; elles circulent et influencent le positionnement et le comportement des individus avec leurs interlocuteurs. Nous nous intéressons ici aux images des langues dans la société mexicaine et à la façon dont elles sont perçues, représentées ou valorisées par des enseignants de français langue étrangère (FLE). Divers exemples sélectionnés seront utilisés pour montrer l'importance des images au Mexique. Les notions d'image-histoire, de hiérarchies imaginées de langues-cultures et des locuteurs seront commentées. II s'agit d'une étude qualitative réalisée dans le cadre de deux thèses de doctorat, à travers les entretiens d'un groupe d'enseignants mexicains qui explicitent leurs parcours linguistiques et laissent entrevoir leurs opinions.
\end{abstract}

Mots clés : images ; imaginaires linguistiques; FLE ; héritage ; Mexique

\begin{abstract}
Images thatwe can make aboutlanguages, cultures, and speakers result from a construction process between different historical, social, and cultural circumstances. The images are constructed individually, and then they become part of social imagination. These ideas are circulated in society in many different ways; they influence individuals' behaviors when they communicate with other speakers. In this paper, we are interested in language, culture, and speakers' images: historical images and imaginary hierarchies are exposed. This is part of a qualitative investigation done within two Ph.D. theses. We conducted interviews with young Mexican teachers to talk about their experiences in learning foreign languages and their thoughts about the languages they speak.
\end{abstract}

Key words: imaginary; foreign languages; hierarchies; legacy; Mexico 


\section{Introduction}

Le Mexique est le pays hispanophone plus grand de l'Amérique latine, il comporte environ 120 millions d'habitants. II y a, d'une part, des contacts entre l'espagnol et les langues indigènes et, d'autre part, des contacts avec les langues étrangères. Les contacts de langues peuvent avoir des conséquences sociolinguistiques parmi certains groupes, par exemple la diglossie, le déplacement des groupes linguistiques minoritaires vulnérables vers d'autres territoires, ou la mort d'une langue quelconque. Au niveau individuel, il s'agit plutôt de situations de bilinguisme ou de plurilinguisme. Il y a aussi des conséquences psychosociales, qui touchent à des questions identitaires ou bien à la conscience linguistique des individus. Il peut aussi y avoir des conséquences communicatives, telles que l'incommunication ou bien la création de stratégies pour résoudre des problèmes de communication. Enfin, il existe des conséquences politico-linguistiques, qui touchent surtout à la législation, l'éducation, les médias et la culture (Zimmerman, 2004). Partout au Mexique, on peut trouver des communautés fragilisées. La vulnérabilité linguistique se manifeste de plusieurs manières et à différents degrés; elle est en relation avec les croyances et les idéologies individuelles. Au Mexique, il existe un mépris pour l'Indigène, mais aussi pour les Espagnols en tant que conquérants. Paradoxalement, les Mexicains constituent un peuple métis né de la colonisation espagnole au XVI siècle. À l'heure actuelle, le métissage ne correspond pas uniquement aux métis biologiques, mais relève plutôt du métissage culturel (Zimmerman, 2004). Ainsi, la plupart des Mexicains sont métis. Les Indigènes voient chez les métis des traitres qui ont du sang espagnol ; et ceux qui se « sentent » Espagnols et qui déclarent ne pas être indigènes voient le métis comme inférieur à cause de son sang indigène, mais renient aussi la langue espagnole (Zunzunegui, 2013). Je me suis donc demandé si le «mépris » pouvait se rapporter aux langues, aux locuteurs. Est-ce que cela crée une survalorisation des langues étrangères?

D'une part, l'Institut national des langues indigènes (INALI, Mexique) dit qu'un locuteur sur 5 se reconnait comme faisant partie d'une communauté indigène mexicaine. Ce qui correspond à 21,5\% de la population totale du pays (INEG| ${ }^{1}$ ). D’après cette information, 6 Mexicains sur 100 parlent une des 68 langues indigènes ou bien une variante linguistique nationale; ce qui représente 7382785 personnes qui parlent une autre langue que l'espagnol. 51,3\% sont des femmes et 48,7 \% sont des hommes ${ }^{2}$.

D’autre part, il y a au Mexique des contacts avec des langues étrangères. D’après une enquête mexicaine ${ }^{3}$, les six dernières années, le pourcentage d'adultes parlant une langue étrangère au Mexique a augmenté de $9 \%$ à $13 \%$. Les Mexicains de moins de trente ans parlent plus de langues étrangères que ceux âgés de plus de cinquante ans. Ce qui veut dire que les nouvelles générations de Mexicains

1. Information disponible sur www.inegi.org.mx/. [Page consultée le 20 octobre 2020.]

2. Information disponible sur www.inali.gob.mx/. [Page consultée le 20 octobre 2020.]

3. Information en ligne sur www.totaluni.com/archivos_articulos/Mexicanos_y_los_Idiomas_59.pdf. [Page consultée le 10 novembre 2020.] Traduction personnelle. 
ont davantage la connaissance d'une langue étrangère. La formation et le niveau socioéconomique sont en relation avec l'apprentissage d'une langue étrangère. $38 \%$ des participants de l'enquête qui étudient en licence (c'est-à-dire font des études à l'université) ont déclaré la pratique d'une autre langue, tandis que $33 \%$ des participants de haut niveau social ont dit parler une autre langue. L'apprentissage d'une autre langue est plus probable en ville (16\%) qu'à la campagne (4 \%). La population de la région nord déclare plus souvent parler une autre langue (23\%) que celle de la région centre du pays (15\%).

L'objectif de cet article est de présenter un panorama de la diversité linguistique et culturelle mexicaine, ainsi que d'exposer la problématique actuelle de la situation mexicaine. À travers quelques images « héritées » et quelques extraits faisant partie des témoignages, j'essaierai de mettre en évidence certaines « hiérarchies imagées » sur les langues-cultures et les locuteurs. Les hiérarchies imagées (Torres-Castillo, 2018: 125) sont des représentations individuelles. Elles sont inscrites dans des jugements de valeur, des croyances et dans les manières de faire et de dire (Amossy et Herschberg, 1997).

Il existe plusieurs idées reçues à travers le temps sur les langues au Mexique, ainsi que quelques stéréotypes autour des Indigènes, des Européens ou des Mexicains. Une communauté a un ensemble de formules stéréotypées adaptées à différentes circonstances préétablies. La culture de la communauté est inscrite dans la langue, c'est elle qui émet des valeurs symboliques de cette culture (Gschwind-Holtzer, 1981). Le stéréotype est une idée restant ancrée dans la mémoire collective, il est aussi générateur d'une idée sur la communauté linguistique d'appartenance du locuteur. Au Mexique, on peut constater que le regard sur l'étranger a changé à travers le temps, mais que le regard péjoratif envers les Indigènes est resté ; d'ailleurs ces locuteurs deviennent vulnérables, car aux yeux de nombreux Mexicains, les Indigènes continuent d'être des sauvages, des païens, des gens sans culture ni éducation, en bref des gens non civilisés. Saenger et Yurén (2006) présentent des cas d'enseignants mexicains qui, d'après elles, adoptent une attitude de confiscation de l'altérité. Cette façon de comprendre l'autre, de l'infantiliser, est une preuve de condescendance et correspond à une fausse compréhension de l'autre. Saenger et Yurén (2006) concluent qu'il prévaut, parmi les enseignants mexicains participant à leur enquête, une attitude d'ouverture acculturante qui désavoue leur propre identité.

Les hiérarchies imaginées au Mexique vont donc opérer dans au moins deux sens. D’une part, les Indigènes ou ceux qui s'y identifient de par leurs racines indigènes, se sentiraient inférieurs devant ceux qui n'ont pas les mêmes identifications ou les mêmes caractéristiques ethniques. D’autre part, ceux qui s'identifient en tant qu'occidentaux regarderaient de travers les Indigènes et tous ceux qui auraient des traits indigènes. D'ailleurs, Villoro (2005) considère que pour un individu métis comme le Mexicain moyen, il y a toujours un « je » indigène qui, parfois, n'arrive pas à s'exprimer car l'être indigène est indissoluble de son être métis. 


\section{Méthodologie : les images des langues et des locuteurs}

Après plusieurs lectures concernant l'histoire du Mexique, j'ai décidé de réfléchir au contact des langues dans une approche historique. Pour ce faire, j'ai travaillé en tant qu'« insider », c'est-à-dire comme chercheuse partageant plusieurs références culturelles et sociales du contexte mexicain avec les autres enseignants mexicains. Je suis consciente que, parfois, les « étrangers ne puissent pas comprendre nos fatigues et nos poussières ${ }^{4}$ », non pas par défaut du regard, mais plutôt à cause d'une vision différente des choses. Certes les images sont des représentations, mais j'ai choisi la notion d'image car je la considère comme plus appropriée dans le contexte mexicain. Le Mexique est un pays de mythes et de légendes où, depuis les temps anciens, « les images pouvaient se lire, et de ce fait réduire des mots, il y avait un discours pictural qui fonctionnait en parallèle au discours oral, on pensait aussi à travers des images » (Johansson, $2014: 61)^{5}$. L'image ou les images que l'on peut se faire d'une langue et de ses locuteurs sont le résultat d'un processus de construction à travers différentes circonstances historiques, sociales et culturelles (Torres-Castillo, 2018). Ces images des langues sont constituées d'imaginaires « fruit[s] du discours ambiant » (Dabène, 1997: 19). Les images des langues correspondent souvent à des images collectives qui « sont transmises par tradition » (Gülich, 1997 : 36). On peut considérer que les images des langues sont un ensemble de croyances, c'est-à-dire ce que l'on croit savoir des langues et des locuteurs.

Le choix d'explorer les images sur les langues à travers la rencontre entre Malinche et Cortés au XVI siècle m'a permis de considérer certains des aspects historiques qui constituent l'héritage imaginaire des enseignants mexicains. D'ailleurs, les images sont vues ici comme des « émergences d'une expérience antérieure » (Wunenburger, 2003: 57), une sorte d'expérience revécue par les individus.

\subsection{Les statuts formel et informel des langues au Mexique}

D’après Dabène (1997), les langues existent dans deux espaces. Le premier espace est représenté par l'usage concret qu'une société fait des langues. Il est constitué par les dispositions officielles à caractère juridique qui régissent l'emploi et l'enseignement des langues. Cet espace est aussi constitué par les politiques linguistiques qui encouragent l'utilisation d'une langue ou qui permettent aux locuteurs l'utilisation d'une langue particulière dans un contexte précis. Le deuxième espace est celui qui se réfère au discours des membres du « corps social » (étudiants, institutions, familles et enseignants).

Le statut informel des langues se rapporte à la subjectivité de l'individu, qui s'approprie différemment la ou les image(s) de langues et des locuteurs. Pour comprendre cette « réalité » imaginaire, je me suis basée notamment sur trois aspects de l'épistémologie multiculturelle de Semprini (1997).

4. Adaptation personnelle du texte de Garro (2003: 350).

5. Traduction personnelle. 
- «La réalité est une construction » (p. 59). C'est-à-dire que pour comprendre les statuts informels, il faut tenir compte des histoires personnelles des Mexicains.

- «Les interprétations sont subjectives » (p. 59). Plusieurs valeurs transmises par héritage culturel sont des constructions intersubjectives. Les apprenants de français possèdent diverses hiérarchies imaginaires. Ce sont des interprétations subjectives qui, parfois, ne reflètent pas la réalité partagée par tout le monde.

- «Les valeurs sont relatives (p. 59). Pendant plusieurs années, dans les formations de FLE, le public mexicain s'est vu attribuer une problématique commune et homogène. Dans les ouvrages de didactique du français, on a tendance à classer les apprenants de FLE comme «non-natifs », avec des caractéristiques communes à tous ceux qui appartiennent à cette catégorie absolue. Cependant, plusieurs valeurs accordées aux langues, aux locuteurs, à l'autre, sont enracinées dans leurs histoires personnelles (Torres-Castillo, 2018).

À travers certaines catégories présentées lors de cette étude, j'ai essayé de découper cette « réalité » et j'ai constaté qu'elle était parfois construite sur des édifices imaginaires.

\subsection{L'étude}

Cette étude de terrain a été en partie réalisée en 2011 et 2012 auprès de jeunes enseignants de français langue étrangère au Mexique. II s'agit d'enseignants formés dans un programme mexicain de licence ou de master en didactique du français dans des universités publiques mexicaines (l'Université de l'État de Mexico et l'Université de Veracruz à Xalapa). Il s'agit d'une étude qualitative dans le cadre de deux thèses de doctorat. Une partie du corpus est constituée d'entretiens semi-dirigés transcrits. Des questionnaires ont été remplis par les étudiants du master en didactique du français de Xalapa en 2012. À ces données s'ajoute l'observation des enregistrements vidéo de diverses classes de français langue étrangère. Parfois, les participants ont répondu en espagnol : dans ce cas, les entretiens ont été traduits en français.

Comme les données ont été recueillies en 2011 et 2012, on peut penser qu'une mise à jour des résultats devrait être envisagée. Cependant, comme il s'agit d'imaginaires collectifs, nous pensons que les idéologies perdurent, qu'elles ne changent pas du jour au lendemain. Les chiffres concernant les enseignants de français ont probablement évolué ces huit dernières années. Ajoutons que plusieurs images sont inscrites dans l'histoire du Mexique. La Conquête du Mexique est, encore aujourd'hui, un thème d'actualité au Mexique. Un événement autour de cette thématique a eu lieu en 2019 lors du 500 anniversaire de l'arrivée de Cortés au Yucatan. Le président du Mexique a demandé au roi d'Espagne et au pape de s'excuser ${ }^{6}$. La prise en compte de cet évènement historique a permis une approche innovatrice. L'étude documentaire s'est faite à travers ce que j'ai nommé « les images-

6. Information en ligne sur https://cubasi.cu/es/noticia/mexico-presidente-pide-disculpa-de-la-iglesia-por-conquista. [Page consultée le 10 novembre 2020.] 
histoire » (Torres-Castillo, 2018: 146). Au fil du temps, ces images ont acquis un caractère de plus en plus important, elles ont été plusieurs fois associées à la magie et à la religion.

\subsubsection{Les images-histoire au Mexique}

Dans l'Antiquité, au Mexique, l'image servait de moyen de communication. Le monde était exprimé à travers des images, car on voyait au travers d'un schéma visuel les personnes ou les objets du monde réel. Cependant, « les Indiens ne partageaient pas la conception espagnole de l'image (...) », « la peinture indigène n'est pas une image à proprement parler. C'est pour une part, un mode de communication graphique soumis à une logique de l'expression et non pas au critère d'une imitation réaliste jouant un redoublement » (Gruzinski, 1990 : 85). De plus, « les codex ont été conçus pour être lus, les codex nahuas maintenaient la mémoire structurée (...) La configuration de l'image ne se soumettait pas à la "tyrannie" des mots mais elle présentait du sens avec une sémiologie propre » (Johansson, $2004: 189)^{7}$.

Les codex mésoaméricains sont élaborés dans une espèce de papier fait à base de liber de ficus nommé amatl (en nahuatl). Les codex mexicains ont été pour la plupart détruits, ou se trouvent dans des collections privées inaccessibles au grand public. D’autres codex ont été produits après l'arrivée des Espagnols au Mexique. Ces codex ont été principalement utilisés pour l'évangélisation. Cependant, dans certains cas, les codex ont servi à des moines comme moyen de préserver cette tradition expressive. Les codex ont été peints par les Indigènes car il existait une tradition de scribes qui racontaient des histoires, des légendes à travers des images. Ces documents ont été faits par des Indigènes sous la « direction » des moines espagnols. Il faut notamment mentionner l'Histoire générale des choses de la Nouvelle-Espagne (Sahagun et Jourdanet, 1880).

Les images qui nous intéressent particulièrement ici montrent les contacts de langues du Mexique. Ce sont celles dont Malinche et Cortés sont les personnages centraux. Il est important de considérer que dans les images-histoire mentionnées ci-dessous, les couleurs ont de l'importance parce qu'une même couleur pouvait signifier des symboles collectifs différents. Les couleurs sont d'ailleurs vues d'une façon différente selon le contexte : Wunenburger (2003 : 64) signale que «les couleurs, par exemple, se voient dotées, dans toutes les cultures, de valeurs affectives et intellectuelles analogues (...)».

\subsubsection{La langue étrangère, un instrument de pouvoir}

El lienzo de Tlaxcala a des caractéristiques hybrides. Ce document présente le récit en détail de la Conquête du Mexique, il montre la vision des Indigènes. Les images contenues dans ce document forment un inestimable livret visuel. C'était aussi un outil pour ceux qui avaient participé à la Conquête du Mexique. Ils pouvaient réclamer de se faire payer pour leurs services à la couronne espagnole (Bueno-Bravo, 2010). Ce document peint par des Indigènes au XVIe siècle a été fait en trois originaux

7. Traduction personnelle. 
qui se sont perdus. Une copie en a été faite par l'association Mesolore ${ }^{8}$. Cette reproduction utilise la lithographie imprimée en 1892 par Chavero.

Les images-histoire présentées ici sont extraites du codex El lienzo de Tlaxcala9. Sur ce document figure le soutien du peuple tlaxcalteca à Cortés pendant la Conquête du Mexique (Nuñez-Rodriguez, 2012). Malinche apparait dans plusieurs scènes; elle est toujours placée comme médiatrice. Alors que les femmes étaient habituellement représentées assises, « elle est peinte debout, l'équivalent de la liberté d'une prostituée » (Thomas et Villeneuve, 2011: 368). On la voit à côté de Cortés; dans d'autres images on la voit transformée (Fig. 1), car elle porte des chaussures espagnoles qui contrastent avec les habits traditionnels des femmes indigènes. Cette image voulait probablement exprimer le fait que Malinche parlait espagnol (Torres-Castillo, 2018).

Figure 1 : El lienzo de Tlaxcala (Image 19/86) ${ }^{10}$

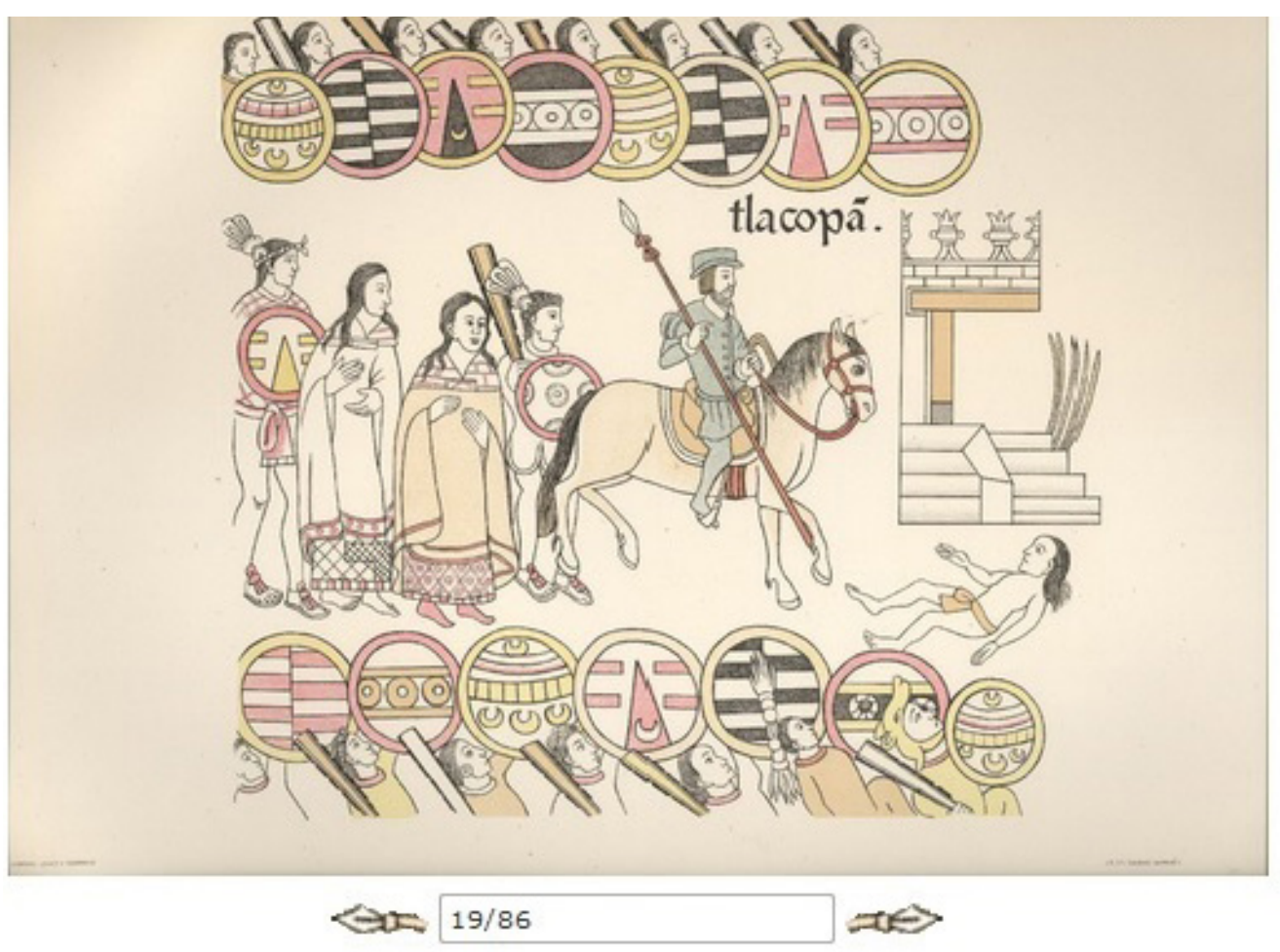

8. Un aperçu de cette copie accompagné d'explications est disponible sur www.mesolore.org/tutorials/learn/19/ Introduction-to-the-Lienzo-de-Tlaxcala. [Page consultée le 10 novembre 2020.]

9. El lienzo de Tlaxcala, explicación de Alfredo Chavero 1892. Information disponible sur http://marcofabr.blogspot. fr/2011/05/explicacion-del-lienzo-de-tlaxcala-por.html. [Page consultée en novembre 2016.]

10. Source : www.mesolore.org/tutorials/learn/19/Introduction-to-the-Lienzo-de-Tlaxcala. 
Dans ce document, Malinche est "présentée d'une taille plus grande » (Fig. 2), parfois les seuls qui détiennent le glyphe représentant la " parole » sont Cortés et Malinche. "Dans une autre image, Cortés est tourné vers Malinche, presque en tant qu’observateur »(Brotherston, 2013 : 11).

Figure 2: El lienzo de Tlaxcala (Image 6/86) ${ }^{11}$

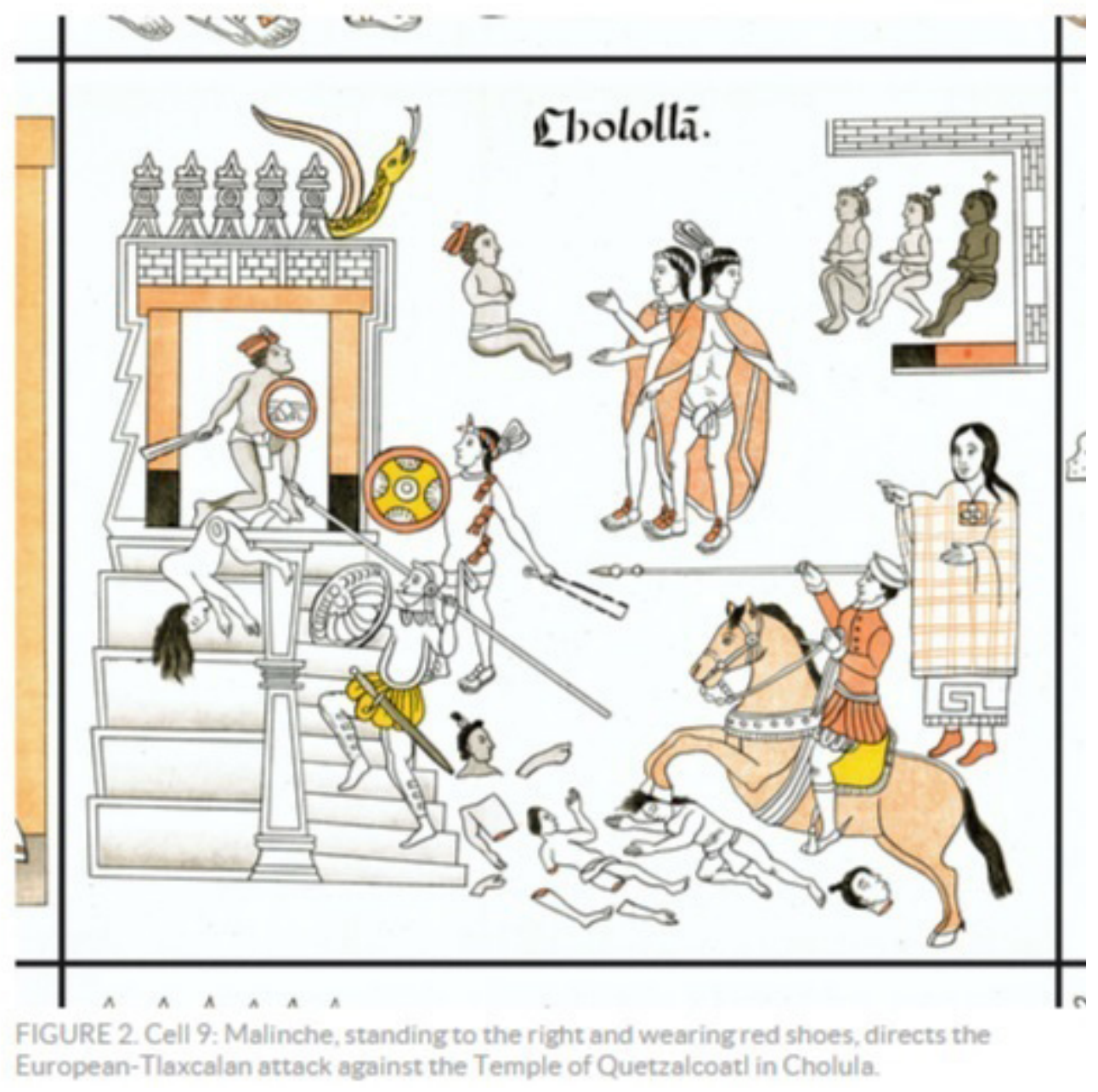

Dans cette image, on peut aussi apprécier la participation de Malinche en tant que médiatrice culturelle. Elle se situe entre plusieurs locuteurs de langues distinctes (nahuatl-maya-castillan).

Même si le mythe autour de Malinche et Cortés est toujours vivant au Mexique, ces deux personnages provoquent encore des controverses. Pendant de nombreuses années, cette femme a été présentée dans les livres d'histoire comme la maitresse de Cortés et la complice de la victoire sur les Mexicains (Bartra, 2013). D’après Zuñiga (2003), les « mémoires historiques » héritées via des écrivains, des chercheurs, conscients ou pas, continuent d'alimenter la domination à travers des mythes.

11. Source: www.mesolore.org/tutorials/learn/19/Introduction-to-the-Lienzo-de-Tlaxcala. 


\section{Diversité linguistique et contacts de langues au Mexique}

Divers contacts entre les langues européennes et les langues indigènes se sont déroulés depuis les premières rencontres, lors de la conquête du Mexique au XVI e siècle. Actuellement, il existe des communautés diglossiques partout dans le pays, par exemple dans la zone du Yucatan ou celle du centre du pays (Zimmerman, 2015). L'espagnol parlé actuellement est le résultat de plusieurs contacts de langues ayant eu lieu au fil du temps au Mexique en tant que colonie espagnole. L'espagnol a intégré plusieurs mots du nahuatl et d'autres langues indigènes (Zimmerman, 2004). Actuellement, l'enseignement de l'espagnol au Mexique suit trois grandes orientations : l'espagnol enseigné aux enfants et adultes scolarisés au Mexique ; l'espagnol enseigné en tant que langue étrangère, destiné aux étrangers non hispanophones; et l'espagnol enseigné aux Indigènes (Torres-Castillo, 2018).

L'anglais en tant que langue étrangère a une grande influence au Mexique, c'est la langue du voisin du nord, les États-Unis. On peut comparer le nombre de locuteurs de langues indigènes, qui s'élève à peine à 7 millions de personnes (connues), aux « 9,5 millions de Mexicains de plus de 18 ans qui déclarent parler anglais. Dans le nord du pays, 1 adulte sur 5 dit parler cette langue, tandis que dans le sud du pays, 1 sur 25 seulement la parle ${ }^{12}{ }^{2}$.

En comparant le nombre de locuteurs de langues indigènes actuel avec celui des locuteurs de l'anglais, on pourrait dire qu'il est normal de trouver un grand écart, puisque l'anglais est une langue internationale avec beaucoup d'influence dans le monde. Mais il faut prendre en considération que le nahuatl a été un temps la langue « officielle » de l'ancien Mexique : elle a été pendant environ 200 ans, entre 1521 et 1771, une langue privilégiée maintenant le fonctionnement de la société coloniale. L’espagnol était plutôt considéré comme la langue étrangère, celle du colonisateur.

Puis le nahuatl a cessé d'être admis dans la Nouvelle-Espagne par un décret royal. Entre 1771 et 1821, l'espagnol est devenu la langue principale de cette colonie (Barriga et Butragueño, 2015). C'est seulement en 2003, avec la création de la Loi des droits linguistiques des communautés indigènes au Mexique, qu'une partie de la population mexicaine parlant une autre langue que l'espagnol a été reconnue. Cependant, la reconnaissance est établie selon un caractère formel et non pas sur le statut informel. Au Mexique, on risque de continuer à croire à cette « vérité historique » présente dans l'imaginaire collectif, selon laquelle l'infériorité du Mexicain devant l'étranger serait réelle. Et par conséquent, on continuera à entretenir le mépris pour les langues et les locuteurs indigènes et l'exaltation de la langue étrangère et de l'étranger.

12. Information disponible en ligne sur : www.totaluni.com/archivos_articulos/Mexicanos_y_los_Idiomas_59.pdf. [Page consultée le 10 novembre 2020.] Traduction personnelle. 
Actuellement, l'offre de cours de langues indigènes dans les centres de langues ou universités est moins importante que celle de cours d'anglais, par exemple. On pourrait même penser que les langues indigènes sont des langues étrangères et lointaines pour certains Mexicains. On peut souvent entendre dire, de façon populaire, « que ces langues ne servent à rien ».

L'enseignement de langues étrangères au Mexique est notamment composé de celui de la langue anglaise, suivi par celui du français. Ces deux langues sont arrivées formellement au XIXe siècle, notamment après l'indépendance de l'Espagne.

Le français a été introduit au Mexique grâce aux contacts commerciaux et à la présence française dans ce pays. Les communautés françaises au Mexique étaient notamment formées d'instituteurs français qui se sont installés au nord du pays. Ces enseignants sont venus pour satisfaire la demande de «bons enseignants » provenant de la classe aisée mexicaine du XIXe siècle (Hernandez, 2005). La bonne qualité de l'enseignement en général, et pas exclusivement celui des langues, était associée et réservée aux étrangers. Le français a été introduit au Mexique comme langue « savante ». On croyait que les connaissances étaient mieux transmises par ces instituteurs étrangers, et que la formation des enfants était ainsi entre de meilleures mains (Torres-Castillo, 2018).

Au Mexique, la diffusion du français langue étrangère a été fortement caractérisée par l'offre de cours de l'Alliance française, ouverte en 1886, et celle de l'IFAL (Institut français d'Amérique latine),

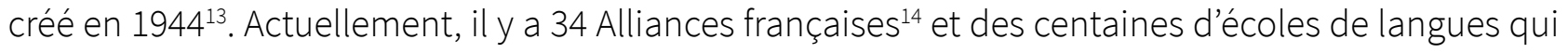
enseignent le français parmi d'autres langues. En 2017, l'ambassade de France au Mexique estime à 250000 le nombre d'étudiants de français sur le territoire mexicain. «L'essor de la langue française au Mexique » est évoqué par Riba (2011) à travers le nombre de candidats aux diverses certifications construites sur le Cadre européen commun de référence pour les langues (CECRL) : en 2010, " 10600 candidats se sont présentés aux épreuves et ont fait du Mexique le $5^{\text {e }}$ pays du monde en termes de nombre de candidats » (p. 48). Les apprenants de français mexicains vivent au moins entre deux cultures et deux langues qui s'entremêlent (Torres-Castillo, 2015). D'ailleurs, la prise en compte d'autres facteurs que la seule compétence linguistique en français, par exemple la diversité des parcours professionnels des apprenants (Goi et Torres-Castillo, 2013), apporte de l'information sur les statuts informels des langues.

\section{Les hiérarchies imaginées}

Les hiérarchies peuvent exister dans des contextes différents et prendre différentes formes. TorresCastillo (2018) mentionne les hiérarchies sociales : le prestige et la légitimité. Dans le cas du Mexique, ces hiérarchies peuvent très bien se présenter à travers le prestige de la langue parlée. Les locuteurs

13. Information disponible sur https://ifal.mx/quienes-somos/qui\%C3\%A9nes-somos. [Page consultée le 25 septembre 2020.]

14. Information disponible sur https://alianzafrancesa.org.mx/ [Page consultée le 25 septembre 2020.] 
d'une langue indigène sont souvent considérés comme des gens sans culture et sans éducation. Parler une langue indigène au Mexique est signe de pauvreté. A contrario, les locuteurs d'une langue étrangère peuvent être considérés comme des individus qui ont plus de prestige social ou appartenant à une élite.

Les hiérarchies culturelles sont établies sur les traditions d'une culture donnée, la culture française est souvent considérée comme dominante ou «meilleure»; ces constructions hiérarchisées peuvent surgir à travers l'origine culturelle ou religieuse.

Les hiérarchies géopolitiques, en relation avec l'origine géographique de naissance, forment une croyance politique; il en va ainsi de la supériorité du locuteur natif d'une langue. Pour les locuteurs du français, on peut utiliser l'exemple du français de la Touraine, qui est réputée parmi les Français comme « la région où l'on parle le meilleur français en France ». À ce titre, Castellotti (2006 et 2011) mentionne les idées connotées par l'accent de Tours : les témoins disent qu'il s'agit d'une « prononciation impeccable », d'une «prononciation parfaite », de la « meilleure prononciation », de l'endroit où les étudiants peuvent apprendre « sans accent », où il y a «l'accent pur », le « vrai accent », ou «l'accent authentique».

Extrait de l'entretien de Minerva, lorsqu'elle parle de l'accent de son enseignante française, qui parle en espagnol ibérique (Torres-Castillo, 2018 : 360) :

8-B Comment a été ton expérience d’apprentissage (du français) ?

9-M ç’a été intéressant et en même temps difficile car la professeure (française) que j’ai eue pendant le premier semestre parlait très peu espagnol et en plus c'était de l'espagnol ibérique, quand elle devait nous donner des exemples ou nous expliquer quelque chose, c'était difficile pour elle de le faire en espagnol, alors elle commençait à parler en français, elle se servait des gestes et des images pour nous expliquer.

Cette même participante a répondu ainsi à la question qui demandait pourquoi elle n'enseignait pas l'espagnol ${ }^{15}$ :

Pour moi, devenir enseignante de français est une très belle expérience, mais en même temps est un défi car il ne s'agit pas seulement d'enseigner l'usage de la langue mais aussi de la culture de France et des pays francophones. J'ai eu l'occasion d'enseigner l'espagnol dans le centre de langue et même s'il s'agit de ma langue maternelle, je ne me suis pas sentie à l'aise, en tout cas pas avec la même aisance que j'ai quand je fais des cours de français.

L'enseignante qui déclare ceci dit aussi avoir été conquise par la musicalité de la langue française (Torres-Castillo, 2018 : 355). Pour Minerva, les sons d'une langue exprimés à travers les accents sont des traits importants pour établir ses propres hiérarchies de valeurs.

15. Traduction personnelle. 
Les hiérarchies sont nées entre colonisés et conquérants; par exemple des hiérarchies naturelles ou biologiques, fondées sur l'apparence physique et « la race ». Il existe également d'autres hiérarchies construites sur l'ethnie ou sur la capacité cognitive; et aussi des hiérarchies de genre, organisées autour des différents rôles entre les hommes et les femmes. Plusieurs de ces hiérarchies peuvent être clairement mises en évidence à travers les exemples présentés ci-dessus (les images-histoire).

Pendant la colonie espagnole, le pape a autorisé les mariages des Espagnols et des Indigènes, avec l'idée que ce métissage permettrait la substitution d'une ancienne âme par une nouvelle (Torres-

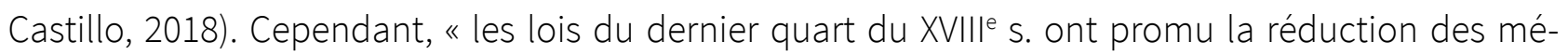
langes raciaux et surtout, ont essayé de garder les éléments de distinction des groupes dominant ${ }^{16}{ }^{\text {}}$ (Gonzalbo, 2012: 125).

Au Mexique, «la plupart des métis» sont nés avec le stigmate de l'abandon paternel (Ramírez, 2005: 239), avec l'image d'une mère indigène soumise qui a juste satisfait le besoin sexuel d'un homme. L'image du père est associée à la force et à l'infidélité acceptée par la société. Les enfants des métis ont donc grandi avec des images stéréotypées des femmes et des hommes. Ramos, cité par Uranga (2005), dit que c'est au moment de la Conquête que le complexe d'infériorité (présupposé chez les Mexicains) est né.

Les hiérarchies professionnelles et d'apprentissage sont tantôt jugées en termes de performance dans le métier, tantôt construites autour du prestige de la formation professionnelle ou de l'expérience en enseignement et la transmission d'un certain savoir. Elles peuvent être présentées à travers les différentes images qu'on se fait du bon enseignant du français. On pense souvent que le locuteur natif est plus compétent en tant qu'enseignant et que le locuteur non natif est un mauvais enseignant, car il a un accent et ne maitrise pas parfaitement le français.

En ce qui concerne les hiérarchies en relation avec les capacités ou les aptitudes dans l'enseignement des langues autour des compétences à enseigner, on privilégie parfois uniquement la compétence orale et on évalue très facilement les compétences d'une personne à travers la présence ou pas d'un accent étranger.

\subsection{Comprendre l'autre à travers des hiérarchies imaginées}

Charaudeau (1995) explique comment une « force souterraine » nous entraîne à essayer de comprendre l'autre. D'une part, on se découvre différent devant l'autre et on se découvre « incomplet, imparfait, inachevé » (p. 16). D’autre part, on se construit des hiérarchies imaginées avec lesquelles on cherche à savoir si l'autre est supérieur ou s'il représente une menace pour soi. Le rejet de l'autre est plutôt exprimé lorsqu'on le voit comme une menace.

16. Traduction personnelle. 
Les préjugés, les clichés ou les stéréotypes concernant l'autre sont plutôt vus comme une nécessité pour se protéger de l'autre, « ils constituent d'abord une protection, une arme de défense contre la menace que représente la différence » (Charaudeau, 1995 : 17). La rencontre avec l'autre, vue par Charaudeau, fait surgir un paradoxe chez l'individu (Torres-Castillo, 2018). L'autre serait jugé négativement (trop froid, rationnel ou agressif), car on se considère comme « meilleur », car on est persuadé que l'on est exactement son contraire. Nous serions convaincus que « nos normes sont les seules possibles » (Charadeau, 1995 : 17), l'autre serait alors en défaut car il ne suit pas nos normes. À titre d'exemple, imaginons qu'un individu juge qu'il parle correctement le français : c'est à la fois en fonction de ceux qu'il voit comme un modèle idéal (tel celui du locuteur natif) auquel il cherche à s'assimiler, et de l'autre qui devient alors celui qui ne maitrise pas suffisamment bien la langue française.

Il apparait évident que les diverses hiérarchies empêchent les bonnes relations avec les autres, " parce qu'il s'agit plutôt de constructions négatives qui changent la réalité et dès lors la façon d'agir. Peut-être qu'elles empêcheront de se surpasser ou de s'améliorer » (Torres-Castillo, 2018 : 129). Les hiérarchies imaginées deviendraient des obstacles à la découverte de l'autre ainsi que des chaines de domination plus ou moins acceptées. Les hiérarchies imaginées sont à la base des raisons par lesquelles s'expliqueraient plusieurs comportements glottophobes et situations de vulnérabilité linguistique, qui atteignent tous les locuteurs à un moment donné. S'agissant des images individuelles, les hiérarchies imaginées sont des façons de voir la réalité et surtout des conditionnements dans l'approche de l'autre.

\subsection{Hiérarchies imaginées de locuteurs}

Charaudeau (1995) et d'autres chercheurs ont remarqué que la valeur positive ou négative varie selon la situation du groupe qui répond. Ils ont donc utilisé la psychologie sociale, notamment pour expliquer les catégories sociales et les rapports de domination. Il y a une certaine tendance à l'assimilation (des « inférieurs » aux « supérieurs »). Les individus s’approprient des qualités positives de l'autre groupe prestigieux (« inférieurs » vers les « supérieurs»), c'est ce que Charaudeau appelle « assimilation positive ». Mais il existe aussi une « assimilation négative », lorsque les « supérieurs » perdent des qualités positives (p. 26). "Un autre phénomène appelé "substitution" serait celui de garder ses valeurs et s’attribuer les qualités des autres, qui sont censés les perdre » (p. 27).

Prenons comme exemple ce que Gadet et Ludwig (2015) décrivent comme une « longue tradition de condescendance » (p. 47) : «La condescendance se voit dans des moqueries et des jugements négatifs sur la façon dont ces autres locuteurs s'expriment en français » (p. 45). Ceux qui se pensent supérieurs disqualifient les locuteurs à travers une série de termes « péjoratifs ». Plusieurs fois, la suprématie imaginée repose sur une supposée légitimité, sur un modèle de locuteur imaginaire. Quelques exemples sont présentés par Gadet et Ludwig (2015 : 45-46) : « Le terme baragouin (dérivés baragouiner, baragouinage) qui désigne à l'origine les Bretons parlant mal le français ». Un autre 
exemple serait celui de "petit-nègre », «terme qui désigne des variantes du français jugées dégradées ou inférieures, supposant des représentations caricaturales, dévalorisantes, racistes ». À travers ces termes, la langue parlée par ces individus est intégrée en même temps à une échelle de valeurs. Parler un « petit français », "français cassé », « français-façon » (p. 47), ou un français comme celui des Français du sud s'exprimant dans une langue française régionale et avec un accent particulier, entraîne une qualification de "campagnards ». Ces locuteurs sont en même temps caractérisés comme inférieurs car ces individus ne correspondent pas aux modèles imaginaires du locuteur. Ces situations peuvent se reproduire aussi chez les apprenants du français, ou bien chez les allophones, à qui on collera l'étiquette de «non-natif » et donc ayant des soucis de compréhension en français: peu importe leur niveau de maitrise de la langue étrangère aux yeux de différentes personnes, ils auront une image dégradée et inférieure.

Pendant la colonisation espagnole au Mexique, ceux qui se sentaient « supérieurs » ont agi avec la même condescendance envers les Indigènes. Leur façon de traiter les Indigènes se justifiait sur la base de la foi chrétienne, la manière de s'adresser aux autres était donc particulière car il fallait respecter certains protocoles. La condescendance envers les Indigènes s'est en quelque sorte muée en domination extrême, justifiée par le vasallaje et l'encomienda : par le prétexte d'aider les Indigènes à se corriger et à devenir des « personnes » civilisées.

Le Mexicain «modèle » est décrit à partir de plusieurs critères. II s'agit de traits caractéristiques qui n'échappent pas à l'œil des Mexicains. Physiquement, il a la peau brune, bronzée, les cheveux très raides. Quelquefois, les traits physiques sont plus indigènes ou bien plus européens. Dans ce cas-là, on peut trouver des distinctions et même de la discrimination envers ceux qui ont des traits indigènes. L'apparence physique correspond aussi à la façon de s'habiller et même de se tenir. Souvent, on associe un comportement à la « race».

Alors que tous les habitants du pays sont actuellement des métis, il existe du mépris et de la distinction. Il y a encore aujourd'hui des expressions qui décrivent des comportements autrefois associés aux Indigènes. Se faire appeler « indio » ou «naco » est une insulte qui implique le manque de goût et d'éducation. L'image des indios est particulièrement distincte dans quelques secteurs de la population mexicaine.

L'indio n'a pas de culture générale, il vit encore attaché à des habitudes et des traditions, et par des liens avec des superstitions. L'indio a de l'amour pour la terre, il n'a pas beaucoup d'émotions, il a seulement des émotions pour ce qui pendant longtemps a été sa passion [...] $\left(\right.$ Chavez, 2002:32) ${ }^{17}$.

En Nouvelle-Espagne, les femmes étrangères (peninsulares) étaient valorisées, elles s'occupaient des activités religieuses et civiques, mais pas de leurs enfants, «criollos». Pour s'occuper des enfants, il y avait les « Marias de a peso », des femmes indigènes. La « nana », femme indigène, était dévalorisée

17. Traduction personnelle. 
par sa condition indigène (Ramirez, 2005). Les premières années dans la Nouvelle-Espagne étaient sous la coupe des criollos (Espagnols nés en Amérique). Les Indiens « ont été exploités par tout le monde, par le propriétaire criollo et par le fonctionnaire métis» (Villoro, 1996 : 212).

Le modèle du locuteur fantasmé est celui du locuteur « natif », habituellement défini comme « sujet parlant sa langue maternelle et considéré à ce titre comme l'ayant parfaitement intériorisée, donc capable de jouer le rôle d'informateur » (Galisson et Coste, 1976 : 366). Il y a plusieurs choses à dire autour de ce modèle : diverses hiérarchies imaginaires peuvent surgir à travers cette notion. En fait, la promotion du monolinguisme à travers un modèle fantasmé serait plus populaire que celle du plurilinguisme.

Extrait de la réponse de Lorena à la question n 3 du questionnaire de Xalapa, lorsqu'on l'interroge sur sa posture en tant qu'enseignante «non native»:

Il y a des personnes qui pensent que l'enseignement de la L2 est meilleur quand on apprend avec un natif. Moi, je dis que les natifs ne sont pas toujours la meilleure option. Bien qu'ils aient la maitrise de la langue, dans plusieurs cas ils ne sont pas formés à l'enseignement et ils n'arrivent pas à expliquer quelques aspects de grammaire/afin que les apprenants comprennent clairement les règles d'usage de la langue/++D'autre part connaitre la langue des apprenants donne des avantages/parce que tu sais quelle est la raison principale à l'origine de leurs fautes/le fait d'être passé par la même expérience te donne des outils pour pouvoir les aider. (Torres-Castillo, 2018 : 349)

Contrairement à ce que plusieurs enseignants mentionnent pendant l'enquête, l'enseignant « natif » peut lui aussi expérimenter des moments d'insécurité linguistique, car le «natif» n'est pas toujours sûr de lui, cela dépend de la situation d'enseignement, du niveau et de l'âge des apprenants.

Cependant, au Mexique, le locuteur « natif » du français est survalorisé, ceci grâce au statut informel des langues ancrées dans les imaginaires collectifs. Le « natif » occupe une place privilégiée dans plusieurs écoles et parmi le grand public ; la qualité de l'enseignement est plusieurs fois associé à l'accent de l'enseignant, la plupart du temps la prononciation gallo-française ou celle de « Paris » est la mieux acceptée. Les capacités et compétences pour le travail se voient réduites à des questions phonologiques, un accent imaginé est accepté en tant que norme ou comme standard linguistique. Il ne s'agit pas uniquement d'une discussion sur les pratiques de recrutement au Mexique, mais plutôt des favoritismes existants et du manque de justice sociale, celle-ci comprise comme la possibilité d'accéder à un emploi. 


\subsection{Hiérarchies imaginées de cultures}

Parmi les témoins, on a pu distinguer une certaine valorisation de la culture française, même si les témoins n'ont pas dévalorisé/méprisé leur culture d'origine. Ceci prouve l'existence des hiérarchies individuelles. Elle peut s'expliquer à travers un texte qui servait de source pour l'enseignement au Mexique. Voici la traduction personnelle de quelques extraits de l'œuvre de De la Torre (1893 : 219221)

C'est le pays des excès mais celui-ci est couvert d'une voile délicate et amusante : les femmes ont la douceur du langage qui au début dérange, mais au bout de quelque temps attire et séduit d'une façon irrésistible, elles ont une élégance et aisance extraordinaires pour porter ces habits, même s'ils sont laids et conservateurs, chacune d'elles porte ce qui est à la mode (...) En un mot, la femme française sait gagner de l'argent et elle sait aussi économiser et épargner.

Mais pour une raison étrange et malgré ces compétences, à Paris il n'y a pas de foyers, il n'y a pas de famille (...) Quand il y a des enfants ils vont habiter loin des parents ${ }^{18}$.

De la Torre (1893) présentait probablement une introduction au « savoir-vivre » d'autres pays à travers son document utilisé comme livre de textes des écoles primaires pour des filles mexicaines. Ces filles devaient apprendre ces préceptes pour pouvoir appartenir à la haute société. Il faut mentionner que les filles qui avaient accès à une formation formaient une minorité privilégiée. On peut supposer que les modèles imaginaires de l'époque étaient inspirés des Européens, car en 1893, on vivait au Mexique sous la dictature de Porfirio Diaz, qui adorait la France. De plus, le ministre des Finances était alors un Franco-Mexicain nommé José Yves Limantour. Lui et sa famille représentaient à l'époque le raffinement de la classe haute mexicaine. La culture française était alors exaltée parmi les autres nations. Il n'est donc pas très étonnant de lire que la « France est le pays civilisé par excellence, et où on peut trouver des gens élégants et qui montrent une grande aisance extraordinaire, c'est aussi le pays qui fait très attention aux bonnes manières » (De la Torre, 1893 : 219).

Par exemple, l'auteur donne aux femmes françaises l'incroyable pouvoir de séduire à travers leur façon de parler. Les femmes mexicaines et françaises sont comparées. Une certaine hiérarchie s'établit puisque la femme française a ce pouvoir parce qu'elle parle en français, tandis que la femme mexicaine n’a pas cette capacité. Le texte a été écrit par un homme, et il semble appartenir à un imaginaire masculin de l'époque. Dans le même texte, vers la fin de son récit, De la Torre fait une comparaison et une sorte de remise en question de l'importance de la famille mexicaine. Il affirme « qu'à Paris il n'y a pas de famille (p. 221) [une autre image fantasmée, car les Françaises sont-elles originaires uniquement de Paris?].

18. Extrait du texte espagnol. Traduction personnelle. 
Chez Charaudeau (1995), on peut aussi voir comment les imaginaires autour des femmes mexicaines coïncident encore avec ceux qu'on avait à l'époque coloniale, où la femme indigène était soumise par tradition. Les imaginaires concernant les femmes françaises coïncident également avec ceux utilisés dans l'éducation basique au Mexique, présentés par De La Torre.

Dans l'étude de Charaudeau (1995), les Mexicains pensent que les Français sont ou se croient supérieurs. Les femmes mexicaines pour leur part pensent que les femmes françaises sont supérieures (p. 40).

Les enseignants de français interviewés ont aussi leurs idées sur la culture française, ces idées sont inscrites dans leurs propres expériences quotidiennes avec les locuteurs de cette langue, ou bien à travers leurs vécus et aussi via les imaginaires collectifs.

(...) « La culture française est celle qui a plus apporté à l'humanité, à travers des manifestations artistiques, sociales, politiques, sportives, etc. Je considère qu'il est vraiment nécessaire que les étudiants puissent connaitre la culture française. » Exemple 5 : Extrait de la réponse à la question 2 de la participante Fany, de Xalapa, (traduction personnelle) lorsqu'elle parle de la «culture française ». (Torres Castillo, 2018 : 282)

"Le français est une langue officielle et d'une certaine manière, la langue de la culture. » Exemple 13 a : Extrait de la réponse de Viridiana à la question 1 du questionnaire de Xalapa (traduction personnelle), lorsqu'elle parle de la culture. (Torres Castillo, 2018 :293)

Ces déclarations mettent en valeur la «culture française ». Elles nous inciteraient à penser que le pays où se « produit » la « culture » « véritable » est la France, ou bien que c'est le pays où l'on s'intéresse par tradition à la «culture cultivée ». On peut faire l'hypothèse que la participante ne place pas sa culture d'origine au premier rang (Torres Castillo, 2018). Ces exemples nous permettent de voir que ces enseignants ont placé la culture française en accord avec des hiérarchies imaginées individuelles.

Parmi les participants de Xalapa, Jaime, María et Fany sont d’accord, ils pensent « qu'à travers la langue on découvre un nouveau monde ». Dans le même sens, les participants Illse, Sandra, et Viridiana pensent qu'avec « une langue on peut apprendre une culture, voyager et acquérir de nouvelles connaissances». (Torres Castillo, 2018:300)

Dans cet exemple, la langue est liée à la «culture française ». Les participants expriment leur admiration et leur enchantement pour la langue française, peut-être au détriment de leur propre langue et culture. Peut-être que la découverte n'existe pour eux qu'à travers le dépaysement. Leurs hiérarchies imaginées sont évidentes, car ils cherchent à découvrir d’autres mondes à travers les langues les plus prestigieuses uniquement. À aucun moment ces participants n'ont signalé leur conscience d'appartenir à une société pluriculturelle : il suffirait de se déplacer à l'intérieur du pays pour trouver d'autres langues et découvrir d'autres mondes. 


\subsection{Hiérarchies imaginées de langues}

L'apprentissage de certaines langues est considéré comme une montée dans la hiérarchie sociale. Les langues deviennent des échelons vers le haut ou vers le bas : «Apprendre une langue prestigieuse socialement est la clé de l'ascension » (Torres-Castillo, 2018).

Dans le cas du Mexique, le statut formel et informel de l'anglais est plus favorable. L'anglais s'affiche dans les cours obligatoires de plusieurs établissements scolaires. L'anglais est présenté au Mexique « comme plus prestigieux », comme langue qui assure « la fierté d'être dans le vent de la mondialisation » (Montenay, 2005 : 67). Selon Hernandez (2005), on ne peut pas discuter au Mexique de la situation du français langue étrangère (FLE) sans mentionner la présence de l'anglais, langue aussi enseignée partout dans le pays.

Depuis les années 2000, l'offre de formation en français langue étrangère dans des institutions publiques et privées s'est davantage développée. Le français fait actuellement partie des curriculums dans certains lycées comme dans l'enseignement universitaire. Depuis plusieurs années, la balance est en faveur de l'anglais et de son enseignement en tant que langue étrangère. L'ambassade de France au Mexique estime à 250000 le nombre d'étudiants du français (2013) sur le territoire mexicain.

Les enseignants ont manifesté leur intérêt pour le français car ils pensent cette langue comme un moyen de distinction parmi les Mexicains.

La participante Lorena déclare : "Parler français est avoir du prestige et appartenir à une élite». Exemple 11. (Torres Castillo, 2018 : 292)

Jaime répond «On sait, même si cela parait arrogant, le français n'est pas parlé par tous, c'est une raison élitiste et égocentrique, ma maitrise du français a été très importante et il m'a distingué parmi mes collègues et les autres enseignants de français ». Exemple 12. (Torres Castillo, 2018: 292)

Quand on leur a demandé pourquoi ils ont choisi d'enseigner le français et pas leur langue première, les réponses ont été variables.

« Je ne m'étais jamais posé cette question, pourquoi j'enseigne le français et pas l'espagnol; peut-être est-ce à cause d'un intérêt inné, qui, à travers les années et à l'aide de la licence en français, est devenu ma vocation. L'espagnol me semble aussi une belle langue et encore plus compliquée (...) On est beaucoup à parler l'espagnol, mais peu sont ceux qui ont le courage de parler une autre langue et surtout une langue qu'on n'a pas apprise depuis l'enfance ». Exemple 17 : Extrait de la réponse de Sandra à la question 2 du questionnaire de Xalapa (traduction personnelle). (Torres Castillo, 2018 : 297) 
Le français est mis en valeur, les imaginaires collectifs sont plus valorisants pour la langue française. Elle confirme que plusieurs déjà parlent espagnol.

Reyna répond: " Je n'enseigne pas l'espagnol parce que je ne me suis pas formée dans cette langue, et quand j’ai décidé de choisir ma formation, je n’appréciais pas beaucoup ma langue, mais à ce moment, si je pouvais enseigner aussi l'espagnol, je le ferais et je pense que je le ferais avec le même plaisir que celui avec lequel j'enseigne le français » Exemple 15b. (Torres Castillo, 2018 : 295)

Les remarques par rapport à sa langue première laissent voir qu'elle considère nécessaire de suivre une formation spéciale dédiée à l'enseignement de l'espagnol pour ensuite devenir enseignante. Elle ne considère pas avoir la légitimité en tant que « native » pour l'enseigner.

En ce qui concerne les langues indigènes au Mexique, il est devenu depuis 2003 le droit de tout Mexicain de s'exprimer dans sa langue première, même si celle-ci est différente de l'espagnol. Dans l'article 3 de la Loi des droits linguistiques des communautés indigènes (Ley de Derechos Lingüísticos de los Pueblos Indígenas), l'État mexicain reconnait les langues comme partie du patrimoine culturel et linguistique national. La pluralité de langues indigènes est ainsi établie comme une des principales expressions de la composition pluriculturelle de la nation mexicaine. Puis, dans l'article 4, les langues indigènes sont reconnues dans cette loi en tant que langues nationales grâce à leur origine historique, les langues indigènes ont alors la même validité dans le territoire mexicain. Dans l'article 9, on exprime le droit de tout Mexicain à communiquer dans sa langue sans aucune restriction dans des contextes publics, privés, ou à l'écrit ou oral, et dans toutes ses activités sociales, économiques, politiques ou culturelles (Gouvernement du Mexique, 2003). Malheureusement, comme nous l'avons exposé auparavant, cette belle réalité n'existe que dans le statut formel des langues indigènes. Dans le statut informel, c'est-à-dire le discours des Mexicains, on est loin de considérer les langues indigènes comme moyen d'expression dans la société mexicaine.

\section{Conclusions}

Je me suis aperçue que les images des apprenants sur le Français et sur les autres locuteurs « étrangers » relevaient d'imaginaires nourris d'une sorte d'admiration pour l'étranger. La langue espagnole et les langues indigènes étaient, à l'évidence, moins importantes à leurs yeux. De plus, l'étranger était reconnu comme « autre », mais le plus souvent comme un autre meilleur (Torres-Castillo, 2018). Les images-histoire présentées sont des éléments pour comprendre la réalité passée et actuelle mexicaine. Les locuteurs de langues indigènes sont parfois méprisés ; pas uniquement par les autres, car cette dévalorisation correspond plutôt à une autoévaluation négative. Les Mexicains préfèrent choisir les langues étrangères prestigieuses car elles sont plus valorisées dans la société mexicaine. Cependant, la vulnérabilité linguistique ne se présente pas uniquement pour les locuteurs de langues indigènes. La langue étrangère peut être à double tranchant, c'est-à-dire servir à se mettre en 
valeur et se sentir appartenir à une élite lorsqu'on se trouve entre Mexicains ; ou bien servir comme moyen pour s'auto-dévaloriser devant l'autre-étranger. 


\section{Références}

Amossy, Ruth et Pierrot Herschberg (1997), Stéréotypes et clichés, Paris, Nathan.

Barriga, Rebeca et Pedro Martín Butragueño (2015), Historia sociolingüística de México : México Prehispanico y colonial, Mexico, El Colegio de México.

Bartra, Roger (2013), «Los hijos de la Malinche », dans Margo Glantz (dir.), La Malinche sus padres y sus hijos, México, Taurus, p. 161-164.

Brotherston, Gordon (2013), « La Malitzin de los códices », dans Margo Glantz (dir.), La Malinche sus padres y sus hijos, México, Taurus, p. 7-18.

Bueno-Bravo, Isabel (2010), «El lienzo de tlaxcala y su lenguaje interno », Anales del Museo de América, $n^{\circ} 18$, p. $56-77$.

Castellotti, Véronique (2006), «Accents francophones et appropriation du français : quelles représentations? », dans Actes du colloque Français fondamental, corpus oraux, contenus d'enseignement, Lyon, ENS Éditions, p. 8-10.

Castellotti, Véronique (2011), « Natif, non natif ou plurilingue : dénativiser l'enseignement des langues? », dans Fred Dervin et Vasumathi Badrinathan (dir.), L'enseignant non natif: identités et légitimité dans l'enseignement-apprentissage des langues étrangère, Bruxelles, Éditions modulaires européennes, p. 29-50.

Charaudeau, Patrick (1995), Regards croisés : perceptions interculturelles France-Mexique, Paris, Didier.

Chavez, Ezequiel (2005), "La sensibilidad del Mexicano », dans Roger Bartra (dir.), Anatomia del Mexicano, México, Plaza Janéz, p. 22-45.

Dabène, Louise (1997), « L'image des langues et leur apprentissage », dans Marinette Matthey (dir.), Les langues et leur image, Neuchâtel, IRDP, p. 19-24.

De la Torre, Juan (1893), El amigo de las niñas mexicanas : libro de lectura corriente especial para las escuelas de las niñas, México, Libreria Nueva.

Galisson, Robert et Daniel Coste (1976), Dictionnaire de didactique des langues, Paris, Hachette.

Garro, Elena (2003), La maitresse d'Ixtepec, Paris, L'Herne.

Goi, Cécile et Torres-Castillo, Claudia (2013), « Réflexivité altéritaire et rencontre interculturelle dans un projet de recherche international », dans Véronique Castellotti (dir.), Le français dans la mondialisation, Bruxelles, Éditions modulaires européennes, p. 379-404.

Gonzalbo, Pilar (2012), "Afectos e intereses en el matrimonio en la ciudad de México », Aleph Ciencias Sociales Colégio de México, disponible sur http://aleph.academica.mx/jspui/bitstre am/56789/29225/1/56-224-2007-1117.pdf. [Page consultée en mai 2020.] 
Gouvernement du Mexique (2003), Ley de Derechos Lingüísticos de los Pueblos Indígenas, disponible sur www.gob.mx/cms/uploads/attachment/file/30119/DerechosLinguisticos.pdf. [Page consultée le 11 novembre 2020.]

Gruzinski, Serge (1990), La guerre des images, Paris, Fayard.

Gschwind-Holtzer, Giséle (1981), Analyse sociolinguistique de la communication et didactique, Paris, Didier.

Gülich, Elisabeth (1997), « Les stéréotypes nationaux, ethniques et culturels : une recherche pluridisciplinaire », dans Marinette Matthey (dir.), Les langues et leurs images, Neuchâtel, IRDP, p. 35-57.

Hernández, Magdalena (2005), La politique linguistique et l'avenir du français au Mexique : étude du cas de l'Université de Veracruz, thèse de doctorat, Birmingham, Aston University.

Johansson, Patrick (2004), « Diégesis iconográfica : la gestación semiológica del fuego uráneo en el texto pictórico del Códice Boturini », dans Patrick Lesbre et Marie-José Vabre (dir.), Le Mexique préhispanique et colonial, Paris, L'Harmattan, p. 189-204.

Johansson, Patrick (2014), « Lenguas y literaturas indígenas mexicanas », dans Diego Valades (dir.), Lengua oficial y lenguas nacionales en México, México, AML, p. 50-99.

Montenay, Yves (2005), La langue française, Paris, Les Belles Lettres.

Ramírez, Santiago (2005), "Psicoanálisis del mestizaje », dans Roger Bartra (dir.), Anatomia del Mexicano, México, Del Bolsillo, p. 237-242.

Riba, Patrick (2011), «L'enseignement du français langue étrangère au Mexique : un regard à la lumière des statistiques du DELF et du DALF, certifications officielles du ministère français de l'Éducation nationale », Synergies Mexique, nº 1, p. 47-53.

Saenger, Cony et Yurén, Teresa (2006), "La Mirada del Otro en los Dispositivos de Formación de Lenguas Extranjeras: Isomorfismos de la Política Lingüística y la Mediación », Education Policy Analysis Archives/Archivos Analíticos de Políticas Educativas, vol. 14, n²5, disponible sur www.redalyc.org/articulo.oa?id=275020543025. [Page consultée le 8 avril 2021.]

Sahagun, Fray Bernardino de (1880), Histoire Générale des choses de la Nouvelle Espagne, Paris, G. Masson. [Titre original : Historia general de las cosas de nueva España, 1575-1577. Traduit de l'espagnol.]

Semprini, Andrea (1997), Le multiculturalisme, Paris, Presses universitaires de France.

Thomas, Hugh et Guillaume Villeneuve (2011), La conquête du Mexique, Paris, Robert Laffont.

Torres-Castillo, Claudia (2015), «Enseignants mexicains de FLE "non-natifs": esquisse d'un métier entrecroisé, entremêlé, entrechoqué de plusieurs "cultures linguistiques" ? », dans Emmanuelle Huver et David Bel (dir.), Prendre la diversité au sérieux en didactique/didactologie des langues. Altériser, instabiliser: quels enjeux pour la recherche et l'intervention?, Paris, L'Harmattan, p. 195212. 
Torres-Castillo, Claudia (2017), Las culturas lingüísticas habitus en un mundo plurilingüe, thèse de doctorat, México, Universidad Autónoma del Estado de México.

Torres-Castillo, Claudia (2018), Enseignement du français, altérités et contacts de langues: imaginaires de professeurs mexicains, thèse de doctorat, Tours, France, Université de Tours.

Uranga, Emilio (2005), « Ontología del mexicano », dans Roger Bartra (dir.), Anatomia del Mexicano, México, Plaza Janéz, p. 145-158.

Villoro, Luis (2005), «El yo indígena », dans Robert Bartra (dir.), Anatomia del Mexicano, México, Random House, p. 203-213.

Wunenburger, Jean-Jacques (2016), L'imaginaire, 3e éd., Paris, Presses universitaires de France. [1'éd., 2003].

Zimmerman, Klaus (2004), "El Contacto de las Lenguas Amerindias con el español en México », Revista internacional de Lingüística Iberoamericana, vol. 2, n 4 (El español en contacto con otras lenguas), p.19-39.

Zimmerman, Klaus (2015), «Diglosia y otros usos diferenciados de las lenguas y variedades en el México del siglo XX: entre el desplazamiento y la revitalización de las lenguas indomexicanas », dans Historia sociolingüística de México. Vol. 2 : México contemporaneo, México, El Colegio de México.

Zuñiga, Rosa María (2003), Malinche esa ausente siempre presente, Madrid, Plaza y Valdés.

Zunzunegui, Juan-Miguel (2013), Los mitos que nos dieron trauma. México en el diván : cinco sesiones para superar el pasado, México, Grijalbo. 


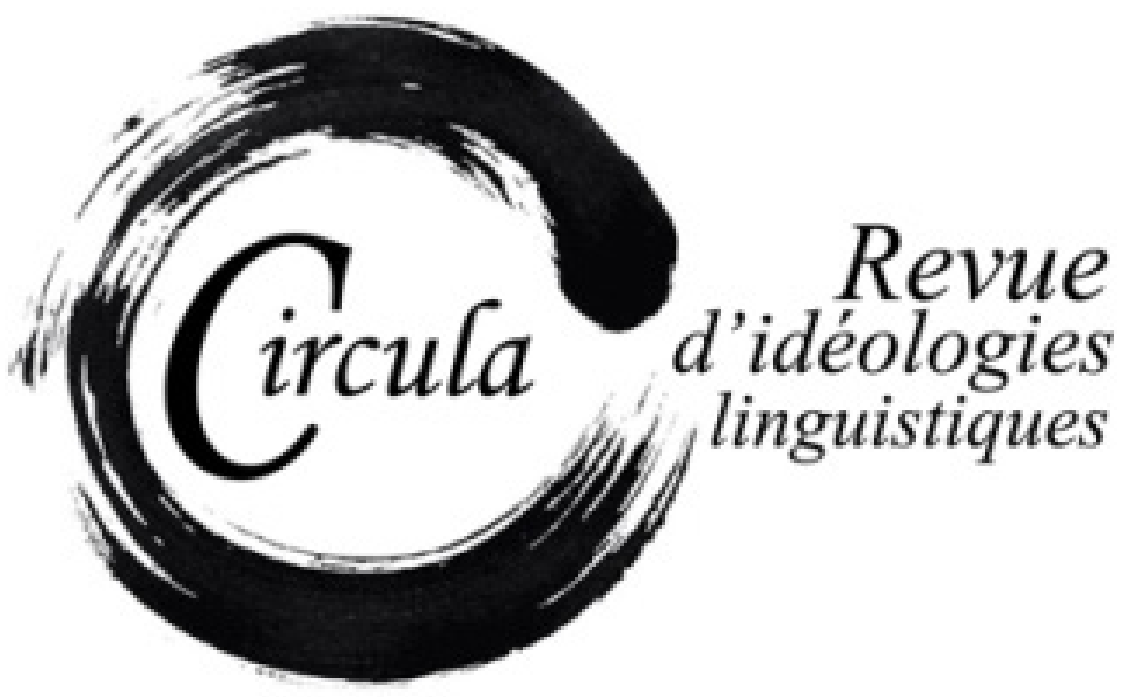

TITRE: "LA GENTE SE RÍE DE NOSOTROS PORQUE HABLAMOS OTRA LENGUA": DISCRIMINACIÓN ÉTNICA Y LINGÜÍSTICA EN LEÓN, GUANAJUATO, MÉXICO

AUteUR: SOPHIA SCHNUCHEL, INVESTIGADORA INDEPENDIENTE ET DAVID CHARLES WRIGHT-CARR, UnIVERSIDAD DE GUANAJUATO

Revue: CIRCULA, NumÉro 12 : LA VULNÉRABILITÉ LINGUISTIQUE

DiRECTRICE: CLAUDIA TORRES CASTILLO

PAGES: $65-86$

ISSN: 2369-6761

URI: HTTP://HDL.HANDLE.NET/11143/18443

DOI: HTTPS://DOI.ORG/10.17118/11143/18443 


\section{"La gente se ríe de nosotros porque hablamos otra lengua": discriminación étnica y lingưística en León, Guanajuato, México}

Sophia Schnuchel, investigadora independiente sophia.schnuchel@gmail.com

David Charles Wright-Carr, Universidad de Guanajuato dcwright@ugto.mx

Resumen: En este artículo los autores presentan los resultados de un estudio de campo llevado a cabo en 2014 en León, Guanajuato, una ciudad industrial del centro norte de México, a partir de las experiencias de 27 individuos pertenecientes a seis pueblos indígenas. Estas comunidades se trasladaron a León, en busca de oportunidades de empleo, durante las últimas cuatro décadas. Se muestran aspectos de las vidas de los migrantes en su nuevo entorno: su vulnerabilidad, el desprecio que experimentan y la discriminación que sufren. Estas personas se encuentran en un espacio intermedio, social y psicológicamente complejo, entre sus culturas tradicionales y el modo de vida de la población mestiza mayoritaria. Aunque hay esfuerzos institucionales para apoyar a los indígenas para que no abandonen su identidad étnica, el menosprecio que experimentan desmotiva el uso de sus lenguas ancestrales.

Palabras clave: idiomas indígenas; multilingüismo; contacto de idiomas; cambio de idioma; discriminación

Summary: In this article the authors present the results of field work carried out in 2014 in León, Guanajuato, an industrial city of north central Mexico, based on the experiences of 27 individuals pertaining to six indigenous peoples. These communities migrated to León, seeking employment opportunities, during the last four decades. Aspects of the lives of these migrants in their new environment are shown: their vulnerability, the contempt they experience, and the discrimination they suffer. These people find themselves in a middle ground, socially and psychologically complex, between their traditional cultures and the lifestyle of the mestizo majority. In spite of institutional efforts to support these communities in the conservation of their ethnic identity, the disparagement they experience demotivates the use of their ancestral tongues.

Key words: indigenous languages; multilingualism; language contact; language shift; discrimination 


\section{Introducción}

En la conformación de la identidad étnica de los pueblos, son esenciales el idioma, el lugar de origen y las tradiciones. Aunque el pasado prehispánico ha sido motivo del orgullo para los mexicanos, los indígenas de hoy se enfrentan a problemas como la pobreza, el aislamiento, la exclusión y la vulnerabilidad. Cuando se alejan de sus lugares de origen, el uso de las lenguas indígenas puede causar el menosprecio y la discriminación hacia sus hablantes. Padecer estas experiencias puede motivar el deseo de asimilarse a la sociedad mayoritaria, para ya no sentirse diferentes. Esto puede motivar el cambio lingüístico y eventualmente el abandono de sus lenguas maternas.

El objetivo de este estudio es determinar si la discriminación étnica sea un factor que cause la pérdida de las lenguas maternas entre los indígenas migrantes en un contexto urbano específico: la ciudad de León, Guanajuato. El análisis se realiza fundamentalmente a través de la recolección y el análisis de las percepciones de los mismos indígenas sobre las experiencias que han vivido, considerando también los contextos histórico, cultural y social, y tomando en cuenta los estudios previos que han abordado problemas similares en esta región. ${ }^{1}$

\section{Estereotipos y discriminación hacia los indígenas en México}

En el México de hoy hay una coexistencia de dos civilizaciones, la mesoamericana y la europea. La relación entre los pueblos indígenas, el "México profundo", y la cultura dominante, el "México imaginario", ha sido conflictiva desde la llegada de los europeos al continente americano. De acuerdo con Guillermo Bonfil Batalla (1990: 11),

1. Este artículo se basa parcialmente en el apartado 5.4 de la tesis de la autora, publicada en alemán (Schnuchel, 2016b). Ha sido enriquecido por la autora con material inédito derivado del mismo proyecto. El autor ha agregado conceptos, ideas y datos adicionales, basados en sus investigaciones sobre la etnohistoria regional, las lenguas indígenas del centro de México y los derechos lingüísticos. Ambos autores agradecen a los evaluadores anónimos de este trabajo por sus atinadas sugerencias, mismas que fueron aprovechadas para mejorar la forma y el contenido del texto.

2. El México profundo, según Bonfil, "está formado por una gran diversidad de pueblos, comunidades y sectores sociales que constituyen la mayoría de la población del país. Lo que los une y los distingue del resto de la sociedad mexicana es que son grupos portadores de maneras de entender el mundo y organizar la vida que tienen su origen en la civilización mesoamericana" (Bonfil Batalla, 1990: 21).

3. El México imaginario se define como "un país minoritario que se organiza según normas, aspiraciones y propósitos de la civilización occidental que no son compartidos (o lo son desde otra perspectiva) por el resto de la población nacional", siendo un sector social "que encarna e impulsa el proyecto dominante" (Bonfil Batalla, 1990: 10). 
Una característica sustantiva de toda sociedad colonial es que el grupo invasor, que pertenece a una cultura distinta de la de los pueblos sobre los que ejerce dominio, afirma ideológicamente su superioridad inmanente en todos los órdenes de la vida y, en consecuencia, niega y excluye a la cultura del colonizado. La descolonización de México fue incompleta: se obtuvo la independencia frente a España, pero no se eliminó la estructura colonial interna, porque los grupos que han detentado el poder desde 1821 nunca han renunciado al proyecto civilizatorio de occidente ni han superado la visión distorsionada del país que es consustancial al punto de vista del colonizador.

En situaciones como esta, es común que se genere un espacio intermedio, en el cual cada uno de los pueblos en contacto busca congruencias culturales, reales o imaginadas, para justificar sus propias acciones. En un proceso creativo de encuentros y desencuentros, comprensiones y malentendidos mutuos, pueden surgir significados novedosos (White, 2011) ${ }^{4}$. Ejemplos de este proceso se pueden encontrar en las obras pictóricas y escultóricas de la época Novohispana (Wright-Carr, 2017), en tradiciones populares como las danzas de raíz indígena (Martínez de la Rosa, Jasso Martínez y Wright-Carr, 2016), y en el culto a la Virgen de Guadalupe (Wolf, 2001: 139-146). En todos estos casos llama la atención un espíritu tenaz de resistencia étnica e ideológica que refleja una resiliencia histórica de los pueblos subordinados frente a los grupos hegemónicos.

Según Bonfil Batalla, se produjo una "desindianización" de la sociedad mexicana porque amplios sectores de la población ya no se identifican como indígenas, aunque conserven tradiciones con raíces mesoamericanas. Bonfil define la desindianización como la "pérdida de la identidad colectiva original como resultado del proceso de dominación colonial” (1990: 13). Esta ruptura con el pasado indígena tuvo dos consecuencias: por un lado, el patrimonio indígena fue idealizado, mientras por el otro, paradójicamente, los indígenas vivos fueron discriminados, desvalorizados y marginados (1990: 23-43). Según Carlos Montemayor (2000: 63-64),

el indio que "renació" fue una idea, una invención ya no de europeos, sino de americanos. A partir de entonces nos creemos herederos de una gran cultura prehispánica y la aceptamos sin compromiso con los pueblos que descienden de esas viejas culturas. Como en una especie de esquizofrenia social, abrimos un gran abismo entre la población indígena actual y la prehispánica. Aplaudimos la figura abstracta del pasado y nos avergonzamos del presente. Exaltamos la memoria prehispánica como mestizaje, pero nuestro racismo se pone al descubierto frente al indio real. Celebramos el mundo prehispánico, pero discriminamos a los indios de carne y sangre.

4. White desarrolló el concepto del "espacio intermedio" ("middle ground") para hablar de las interacciones de franceses e indígenas en la región de los Grandes Lagos de los Estados Unidos de América durante los siglos XVII, XVIII y principios del XIX. El concepto adquirió una vida propia en las obras de otros historiadores, quienes lo han aplicado a una amplia gama de situaciones a través del tiempo y el espacio (White, 2011: 11-24). 
Esta discriminación hacia la población autóctona va acompañada de estereotipos. Los indígenas son percibidos como tales, según Bonfil, por marcadores como "las ropas que usan, el 'dialecto' que hablan, la forma de sus chozas, sus fiestas y costumbres". La población no indígena desconoce las realidades de los indígenas, reconociéndolos por medio de prejuicios simplistas: "el indio flojo, primitivo, ignorante, si acaso pintoresco, pero siempre el lastre que nos impide ser el país que debíamos ser" (Bonfil Batalla, 1990: 45).

Un estudio de Felipe Marañón Lazcano y Carlos Muñiz aborda los personajes indígenas ficticios que aparecen en programas televisivos del estado norteño de Nuevo León, revelando la discriminación hacia los pueblos originarios. En estos programas de entretenimiento se observa el estereotipo genérico del indio mexicano, con trajes tradicionales, jugando papeles secundarios y proporcionando momentos cómicos (Marañón Lazcano y Muñiz, 2012: 20-21). La sociedad en general acepta y se divierte con estas representaciones discriminatorias.

\section{Tipos de discriminación hacia los indígenas}

Ricardo Contreras Soto (2014:84-89) distingue tres "indicadores de alteridad" de los indígenas en el estado de Guanajuato: el color oscuro de su piel, asociado con una clase social baja; la forma de hablar, ya sea en una lengua indígena o en un castellano ${ }^{5}$ etnolectal, es decir, con errores gramaticales y acento; y la forma de vestir, que revela la identidad étnica y que a menudo es ridiculizada.

La discriminación se realiza de diversas maneras: mediante gestos y miradas, designaciones con connotación negativa y la violencia simbólica en el trato, llegando incluso al extremo de la violencia física. La discriminación se produce de forma simbólica, ignorando y negando su presencia y creando estereotipos. Según Contreras, algunas personas perciben a las minorías étnicas como objetos de burla, como pobres “con ropa de mala calidad, ropa de indio, de 'naco'”. Los indígenas se encuentran en desventaja en diversas esferas sociales porque están poco familiarizados con las prácticas culturales de la población mayoritaria. Mientras que algunos mestizos se solidarizan con los indígenas, otros participan activamente en la humillación o la toleran. Las autoridades también discriminan a los indígenas, por ejemplo, mediante la expulsión de un lugar de venta o la confiscación de los bienes de los vendedores ambulantes (Contreras Soto, 2014: 89-96).

\footnotetext{
5. En este artículo, los autores emplean la palabra "castellano" para referirse al idioma llamado hoy comúnmente "español”, para reconocer que hay varias lenguas españolas, cada una con el estatus legal de lengua cooficial (Ministerio de Educación y Formación Profesional, 2019). La Real Academia Española, por razones que desconocemos, no se ha adaptado a esta realidad jurídica, como pudo haberlo hecho mediante la restauración del título original de su principal lexicón, el Diccionario de la lengua castellana, que aparecía en la portada de cada edición hasta 1925, cuando fue cambiado a Diccionario de la lengua española (Real Academia Española, 1925: vii-viii).
} 


\section{Estudio empírico sobre la discriminación hacia los indígenas en León ${ }^{6}$}

\subsection{Procedimiento}

Para determinar los niveles de vulnerabilidad, marginación y discriminación de la población indígena en un contexto urbano, en 2014 la autora de este artículo, con el apoyo del autor ${ }^{7}$, realizó una investigación de campo de siete semanas en la ciudad de León, situada en el estado de Guanajuato, en el centro norte de México.

El campo del estudio se limitó al Centro de Desarrollo Indígena Loyola (CDIL), al Centro Educativo Intercultural Nenemi y al asentamiento conocido como la "comunidad mixteca". En el período del estudio, había diez empleados en estos proyectos: cuatro profesores y dos cocineras quienes trabajaban en la escuela Nenemi, así como cuatro personas quienes trabajaban en ambos lugares, ayudándoles con asuntos burocráticos, ofreciendo cursos de informática y organizando sesiones sobre temas culturales. En una entrevista con los responsables del CDIL, la autora explicó sus objetivos y las funcionarias permitieron a la autora entrevistar a un número limitado de indígenas, con la condición de que ella apoyara a la comunidad indígena como voluntaria. El personal del CDIL ayudó a la autora a establecer los primeros contactos; ella preguntó a varias de las personas que vivían en el CDIL si estaban dispuestos a responder a sus preguntas y así obtuvo a sus informantes. La autora también trabajó en la escuela Nenemi y colaboró con los profesores. Los maestros eran quienes elegían a los alumnos para las entrevistas.

Durante la investigación de campo fueron entrevistados 27 indígenas, usando cuestionarios estandarizados. ${ }^{8}$ En la preparación de la investigación y en la elaboración de los cuestionarios, la autora aprovechó los estudios sociolingüísticos de Geiger (2010), Pfeiler (1988), Terborg y García Landa (2011), Weinel (2011), así como los métodos de investigación social empírica de Przyborski y Wohlrab-Sahr (2014). Los cuestionarios para los adultos incluían 55 preguntas sobre conocimientos lingüísticos, educación escolar, uso del idioma y actitudes hacia los indígenas y no indígenas. Los cuestionarios para los estudiantes eran menos complejos, con solo 45 preguntas.

Para realizar el estudio empírico, se aplicó un método mixto con elementos cualitativos y cuantitativos. La recolección de datos se realizó por medio de entrevistas y a través de la observación participativa. El cuestionario contenía preguntas cerradas y abiertas. Durante las entrevistas con los indígenas, los cuestionarios fueron llenados por la entrevistadora. Además, las conversaciones con 13 de los encuestados fueron grabadas. Fueron entrevistados 12 niños de 8 a 13 años, siete adoles-

6. Este inciso se basa parcialmente en publicaciones anteriores de la autora (Schnuchel, 2016a, 2016b, 2017, 2018).

7. Véase Wright, 2016.

8. Véase el apéndice del presente artículo. Para información más detallada, véase Schnuchel, 2016b: 121-140. 
centes y adultos jóvenes de 14 a 28 y ocho adultos de 30 a 53. Los encuestados pertenecían a seis pueblos indígenas: diez nahuas, nueve tarascos, cuatro otomíes, cuatro mixtecos, una mazahua y un tsotsil. Además se entrevistaron a dos profesores indígenas del Centro Educativo Intercultural Nenemi y al representante de los mixtecos en el Consejo Consultivo leonés, Daniel Méndez Cortés?.

En el siguiente inciso, y con base en el estudio de campo que se describe aquí, la marginación y vulnerabilidad de los indígenas que viven en León se trata brevemente. En seguida, se examinan las declaraciones de los entrevistados sobre la vulnerabilidad, la marginación y la discriminación, y se discute si las experiencias de los informantes corresponden con las situaciones descritas por los expertos que han publicado estudios sobre estos problemas sociales. También se aclara qué efectos tienen estos factores externos en las actitudes de los hablantes acerca de sus lenguas y su uso.

\subsection{Indígenas en la ciudad León}

La ciudad de León se encuentra en el noroeste del estado de Guanajuato, al poniente de la ciudad minera de Guanajuato. Fue fundada en 1576 como una villa española, en el territorio de los nómadas guamares y guachichiles, quienes estaban en una situación de resistencia armada contra los invasores españoles y sus aliados indígenas del sur. Catorce años después, la resistencia de los nómadas en esta región fue neutralizada por el gobierno de la Nueva España, con una política de sobornos en especie, presión militar y la fundación de misiones, lográndose la integración de algunos indígenas en la sociedad pluriétnica del naciente sistema colonial y la dispersión de otros hacia zonas de refugio (Gerhard, 1993: 166-168; Tutino, 2011: 79, 83; Wright-Carr, 2014: 18-23).

Debido a la crisis del campo y la pobreza que sufren muchas comunidades indígenas, desde la década de 1981-1990 se observan procesos de migración de indígenas en búsqueda de trabajo, en los cuales abandonan sus lugares de origen y se dirigen a los centros urbanos (Comisión Nacional para el Desarrollo de los Pueblos Indígenas, 2010b). Este desplazamiento convirtió al estado de Guanajuato, y en especial la ciudad de León, en una región receptora. León se distingue principalmente por su producción de artículos de piel y textiles, así como por las industrias papelera y alimentaria. Ha experimentado un fuerte crecimiento económico en las últimas décadas (Jasso Martínez, 2011: 1121; Sommerhoff y Weber, 1999: 354). La población indígena es atraída a León por la esperanza de obtener un empleo y para vender sus productos artesanales.

9. En este artículo se emplean los nombres en castellano de las lenguas indígenas, según están registrados en el Catálogo de las lenguas indígenas nacionales. Estos nombres representan, en realidad, agrupaciones de lenguas emparentadas, con distintos niveles de inteligibilidad interna. Las lenguas indígenas en México se clasifican oficialmente en 364 "variantes lingüísticas", pertenecientes a 68 "agrupaciones lingüísticas" que forman 11 "familias lingüísticas". En esta obra se pueden encontrar las autodenominaciones usadas por los hablantes de cada variante. Según este texto, "La categoría lengua -o idioma-, intermedia en términos de inclusión entre agrupación lingüística y variante lingüística, se define como un sistema de comunicación socializada mediante el cual dos o más individuos que se identifican como o con miembros de una comunidad lingüística pueden codificar y descodificar, en un plano de mutua inteligibilidad, los mensajes orales o escritos que llegasen a intercambiar. Para la aplicación de esta categoría en el presente Catálogo, las variantes lingüísticas deben ser tratadas como lenguas" (Instituto Nacional de Lenguas Indígenas: 2009: 9-15, 29). 
En 1990 se registraron los primeros asentamientos de indígenas en la historia reciente de León, en el barrio Río Escondido, en el extremo sur de la ciudad, cuando mixtecos de Oaxaca y otomíes de Querétaro se establecieron allí (Balam, 2003: 39). Otros siguieron, fundando la comunidad mixteca, que todavía subsiste. Con los años vinieron indígenas de otras regiones a León, entre ellos nahuas de Veracruz y Guerrero, mazahuas del estado de México, tarascos de Michoacán y tsotsiles de Chiapas. En 1998 fue fundado el CDIL y el año siguiente se construyó un conjunto de viviendas en la colonia La Luz, no lejos de la comunidad mixteca, por iniciativa de la Comunidad Loyola y representantes de las comunidades indígenas ${ }^{10}$. Fue el segundo gran conjunto de viviendas indígenas en León. Sin embargo, la mayoría de los indígenas viven dispersos en la ciudad y suelen relacionarse dentro de sus propias comunidades étnicas. Por lo general viven socialmente excluidos, en asentamientos marginados y pobres, a veces en construcciones de madera y cartón, sin drenaje ni agua potable, sin autorización del gobierno municipal. La población indígena cuenta con menor acceso a la educación y al sistema de salud que las personas no indígenas. A menudo padece malnutrición y enfermedades (Jasso Martínez, 2011: 1117-1127; 2015).

Hay información de diversas fuentes sobre la presencia indígena en León. Según los datos censales que eran vigentes cuando se realizó el trabajo de campo, 3191 habitantes de cinco años o más declararon hablar una lengua indígena (Instituto Nacional de Estadística y Geografía, 2010). Según la Comisión Nacional para el Desarrollo de los Pueblos Indígenas (2010a), 8168 indígenas vivían en León, de los cuales 1479 declararon ser bilingües y 1695 no especificaron su competencia lingüística. Huerta Arellano, Beltrán Soria y Aguilar Doncel (2012: 13) señalan que un total de 5844 indígenas en León pertenecían a los siguientes pueblos: otomí (3 239), nahua (1 264), mazahua (818), tarasco (348) y mixteca (175).

Un antecedente de los proyectos sociales en favor de los migrantes indígenas de León fue la Guardería Indígena, fundada en 1996 por la Comunidad Loyola para supervisar, educary alimentar a los niños indígenas mientras sus padres trabajaban. En 1998 esta iniciativa se convirtió en el Centro de Desarrollo Indígena Loyola (CDIL). Ahí viven 15 de los 27 indígenas migrantes que fueron entrevistados como parte del trabajo de campo que se describe en el presente artículo. El acceso es por la calle Río Verde, un camino sin salida que carece de pavimento. Su entorno inmediato, al lado de un refugio para perros y cerca de un basurero, revela la marginación urbana que padecen los indígenas que viven ahí. En este complejo de vivienda hay 20 unidades familiares, varios talleres, una oficina de asesoramiento, el Centro Educativo Intercultural Nenemi y una sala multifuncional que sirve como capilla, biblioteca escolar y sala de cómputo.

10. Hay información sobre la Comunidad Loyola en el sitio web https://comunidadloyola.org/. Esta institución no gubernamental desarrolla cuatro proyectos sociales: (1) Casa Hogar Loyola; (2) Nima: Centro de Promoción de los Derechos Humanos de Niñas, Niños y Adolescentes en Guanajuato; (3) Centro de Desarrollo Indígena Loyola; y (4) Centro Educativo Intercultural Nenemi. 
Las viviendas son casas de aproximadamente 15 metros cuadrados, divididas en tres pequeñas habitaciones. Estas se ponen a disposición de los indígenas de forma gratuita durante un tiempo limitado. Tienen electricidad pero no hay conexión de agua. Las instalaciones sanitarias son compartidas por todos los habitantes. El CDIL es un centro de acogida para los indígenas que migran a León, para que se orienten en la ciudad, se afiancen, establezcan redes sociales y logren alguna base financiera. Según un censo interno, en este lugar vivían 114 indígenas de entre cero y 64 años, 53 de ellos nahuas, 30 tarascos, 18 otomíes, cinco mixtecos, cinco mazahuas y tres sin indicación del pueblo indígena al cual pertenecían (Centro de Desarrollo Indígena Loyola, 2014). Esto hace un promedio de 5.7 personas por unidad de vivienda. Siete de estas albergan a más de ocho residentes.

\subsection{El multilingüismo y la adquisición de lenguas por los indígenas}

Por lo regular, cuando existe contacto entre dos idiomas se produce el bilingüismo (Appel y Muysken, 2005: 1). Se habla de bilingüismo o multilingüismo "si un individuo sabe dos o más idiomas o bien si se hablan dos o más idiomas en una sociedad", aunque puede variar el grado de competencia lingüística. En general se distingue entre el bilingüismo personal y el bilingüismo social (Bollée y Neumann-Holzschuh, 2009: 12).

En la adquisición de la lengua materna, se hace una distinción entre el bilingüismo simultáneo y el bilingüismo sucesivo. Si un niño tiene contacto con dos idiomas desde el nacimiento, esto se denomina "bilingüismo simultáneo" o "adquisición bilingüe de una primera lengua" (Abelló Contesse, 2010: 181). El bilingüismo sucesivo o secuencial se presenta si la segunda lengua se desarrolla solamente después de que la primera lengua sea establecida, es decir, a partir de la edad de cuatro años o más (Kocianová, 2005: 9). Se llama "bilingüismo desigual" al fenómeno en el cual un niño desarrolla mejores competencias lingüísticas en una de las dos lenguas. En niños bilingües se puede producir, además, una fase de resistencia, o sea el rechazo de comunicarse en una de las dos lenguas, lo cual normalmente afecta la lengua que hable con menor competencia o bien la lengua que tenga un menor prestigio social (Abelló Contesse, 2010: 182, 186).

En León, por lo general, la población indígena es bilingüe. Con la excepción de dos alumnos, los informantes declararon haber adquirido primero la lengua indígena y después el castellano, por lo que se trata de bilingüismo sucesivo. La adquisición del castellano se logró generalmente con anterioridad en los niños que en los adolescentes y adultos.

En el primer grupo, definido por la edad de los entrevistados (de ocho a 13 años), un niño y una niña (informantes I-1.11 y l-1.12) declararon haber aprendido el castellano en la guardería a los cuatro 
años ${ }^{11}$. Las informantes $1-1.3$ y $1-2.5$ adquirieron el castellano en la escuela primaria y el informante I-1.9 lo aprendió en el CDIL, a la edad de cinco años. Varios niños comentaron que se sentían incómodos y tuvieron -o seguían teniendo- dificultades para hablar el castellano: "No podía hablar, no podía pedir nada, me sentía avergonzado" (I-1.5); "No podía pronunciar algunas palabras" (I-1.12); "Hay veces no sabe bien lo que habla en español" (I-1.8).

Del segundo grupo (de 14 a 28 años), seis de los siete encuestados declararon haber aprendido el castellano en la escuela intercultural. Una informante lo describió así: "En la primaria, porque los maestros sí nos hablan puro español. [...] La meta que ellos tenían era enseñarnos a hablar español. Los maestros salían de la ciudad y no podían hablar nuestro idioma" (I-2.6). La adquisición se llevó a cabo por pasos y el español fue aprendido como segundo idioma.

En el tercer grupo (de 30 a 53 años), tres de los informantes entraron en contacto con el castellano en la escuela primaria. La informante I-3.8 no asistió a ninguna escuela pero adquirió algunos conocimientos básicos de manera independiente a los 20 años: "Antes no sabe nada. De vender sabemos poquito. No sabe bien bien pero ya más o menos".

En general, el proceso de adquisición del castellano se realizó poco a poco, empezando por la comprensión y la pronunciación de palabras y frases hasta la formación de oraciones completas. Mientras que algunos de los encuestados lograron adquirir conocimientos sólidos del castellano y lo dominaban de manera similar a su lengua materna, las competencias de comprensión y expresión lingüística de otros, sobre todo entre los informantes mayores, eran notablemente limitadas; no dominaban el castellano con la misma fluidez que la lengua materna. En la mayoría de las personas encuestadas, competencias como la pronunciación, el dominio léxico y la gramática eran marcadas de manera etnolectal.

Se observó, además, que los adultos tenían mejores conocimientos de los idiomas indígenas y más problemas con el castellano, mientras que la mayoría de los niños eran bilingües y dominaban el castellano mucho mejor que sus padres, mientras tenían problemas con las lenguas indígenas habladas por sus padres. Los idiomas autóctonos en los niños eran vulnerables y existía el riesgo de que eventualmente desaparecieran estas lenguas en las comunidades indígenas de León.

11. Las claves alfanuméricas usadas para designar a los informantes contienen la siguiente información: la letra 'l' significa 'indígena'; el primer número se refiere a uno de los tres grupos de edad, con los niños - de 8 a 13 años- en el primero, los adolescentes y adultos jóvenes - de 14 a 28 años- en el segundo, y los adultos mayores -de 30 a 53 años- en el tercero; el segundo número designa a los individuos de cada grupo de edad. (Véase el apéndice del presente artículo para una relación detallada de estos informantes.) Aparte de los 27 informantes mencionados en el apéndice, la autora entrevistó a dos profesores, ambos hablantes de lenguas indígenas, del Centro Educativo Intercultural Nenemi. Estas personas compartieron sus conocimientos y experiencias en entrevistas con una estructura distinta, por lo que no aparecen en la tabla con los demás informantes. 


\subsection{La vulnerabilidad de los indígenas en León}

Los migrantes indígenas en León están obligados por las circunstancias a integrarse en un entorno cultural que les es ajeno; tienen que aprender el castellano o mejorar sus conocimientos de esta lengua. Jasso Martínez (2011: 1127) examinó la vulnerabilidad de los indígenas en León e identificó como los problemas principales la discriminación, la cobertura de las necesidades básicas, la vivienda, la salud, la educación, el empleo y la violencia intrafamiliar. A continuación estas problemáticas se presentarán brevemente.

La discriminación es uno de los principales problemas que enfrentan las comunidades indígenas en León. Debido a su situación de migrantes y a la falta de una red amplia de apoyo y solidaridad, se reduce su capacidad de defenderse contra la injusticia, reclamar sus derechos y exigir el respeto que se debe a todos los seres humanos. Como resultado de la discriminación, algunos indígenas se asimilan a la población urbana y desechan las características externas que los identifican, como la vestimenta y el idioma:

Algunos otomíes y tarascos que habitan en León afirman que han tenido que abandonar sus trajes tradicionales y limitar el uso de su idioma en espacios públicos. Incluso algunos han decidido no enseñar a sus hijos el idioma indígena que hablan (Jasso Martínez, 2011: 1128-1129).

Como ya mencionamos, los indígenas de León viven en asentamientos predominantemente pobres y a veces en asentamientos no autorizados. Tienen menos acceso al sistema de educación y salud, y muchos sufren de malnutrición y enfermedades relacionadas con la pobreza y las viviendas precarias. Según las declaraciones de los informantes, la mayoría se dedica a actividades informales, por ejemplo a la venta de flores, figuras de yeso, helados, papas fritas y artesanías. Debido a su bajo nivel escolar, un alto índice de analfabetismo y la falta de formación profesional, se ganan la vida como vendedores ambulantes. Jasso Martínez (2014: 124) describe la situación de las mujeres indígenas en León:

Estas mujeres están más expuestas a la exclusión social y la discriminación, debido a su actividad laboral (la venta en las calles) y a que deben cubrir las necesidades de toda la familia (servicios de salud y educación, abastecimiento de comida y productos básicos). En este sentido se han convertido en sujetas de abuso y de violación de sus derechos humanos, incluso con más frecuencia que los varones. A pesar de que llegan en situaciones de mayor desventaja que los hombres (con menores niveles de educación formal y un conocimiento básico del idioma), con el tiempo van desarrollando habilidades y conocimientos que les permiten insertarse y permanecer en la ciudad (aprenden a hablar el castellano y se vuelven comerciantes). 
La vulnerabilidad de la población indígena en estas comunidades se debe, al menos en parte, a la discriminación étnica que la sociedad mexicana heredó de la época Novohispana.

\subsection{Vulnerabilidad y discriminación según los encuestados}

Ya hemos explicado la situación de vivienda de los indígenas encuestados. Además del alojamiento que se proporciona gratuitamente en el CDIL durante algunos años, varios migrantes son beneficiarios de los programas gubernamentales. Por ejemplo, once familias que viven en este lugar son apoyadas por el programa interinstitucional "Contigo vamos por más oportunidades" (Centro de Desarrollo Indígena Loyola, 2014), implementado para ayudar a las familias que viven pobreza extrema. Con el apoyo del estado de Guanajuato y una dependencia federal, el Secretario de Desarrollo Social y Humano, el programa persigue el objetivo de brindar "una mayor certeza jurídica, desarrollar incentivos a la participación ciudadana, incorporarlos a programas de vivienda y servicios; y de empleo y generación de ingresos" (Irala Burgos, 2008).

Muchos indígenas padecen malnutrición. Las entrevistas muestran que algunos padecen hambre $y$, por falta de recursos financieros, a menudo sólo comen tortillas con chile. Para asegurar que los alumnos del Centro Educativo Intercultural Nenemi reciban una dieta equilibrada, se sirven ahí dos comidas, desayuno y almuerzo, en los días escolares. En 2014 los alumnos del Centro Educativo Intercultural Nenemi fueron examinados por la nutrióloga Ivonne Téllez para determinar su estado de salud. Se encontró que el 11\% de los niños estaban en riesgo de desnutrición, 74\% tenían un peso normal y 15\% tenían sobrepeso. Esta última condición también se debe a la pobreza de los niños: por una parte, las personas que padecen desnutrición en su infancia son más propensas a la obesidad en años posteriores; por la otra, el sobrepeso suele ser el resultado de una dieta desequilibrada (Téllez, 2014: 14).

La autora tenía interés en saber si los indígenas percibían una marginación y una discriminación hacia ellos. Quería averiguar si los informantes cambiaban su comportamiento debido a la estigmatización, adoptando patrones culturales que los llevaban hacia la asimilación por la sociedad dominante. Se les preguntó qué actitudes tenían los no indígenas hacia los indígenas, si los indígenas estaban marginados en la sociedad y si existía discriminación hacia estos. ${ }^{12}$

Ocho informantes, la mayoría de ellos con 14 años o más, indicaron que los no indígenas tenían una actitud negativa hacia ellos ${ }^{13}$. Uno de los encuestados no expresó opinión alguna y tres informantes manifestaron que los no indígenas tenían actitudes en parte positivas y en parte negativas.

12. Las dos primeras preguntas se hicieron sólo a los grupos 2 y 3. Las preguntas se formularon de manera impersonal para que el encuestado no tuviera que hablar necesariamente de sí mismo y no tuviera que asumir el papel de víctima de la discriminación.

13. El informante I-2.2 explicó esta actitud así: "No nos habla". La informante I-2.5 dijo: "Por la forma como tratan a una persona, uno a veces no sabe hablar, y lo discriminan". 
Tres de los encuestados consideraban que los no indígenas tenían actitudes positivas hacia ellos. La informante I-3.2 mencionó que eran bien tratados y apoyados, refiriéndose al personal del CDIL, y la informante I-3.7 dijo que las actitudes en ese momento eran positivas, a diferencia de tiempos anteriores: "La situación ya está mejor, hay una convivencia con ambos lados, con mestizos e indígenas, y ambos lados se dan cuenta que son buenas personas".

La pregunta de si los indígenas estaban marginados en la sociedad fue respondida por once adultos. Nueve se consideraban marginados, mientras que dos indicaron que no lo eran, como explicó el informante I-2.4: "Ahora ya nos tratan igual". El informante I-2.2, quien consideró que los indígenas eran marginalizados, dijo: "Es más difícil para nosotros", y el informante I-3.1 mencionó las dificultades que encontraban en sus terruños: "En el pueblo nos hace falta agua potable, no hay trabajo [...]. Si hubiera más trabajo allá, no estuviera aquí".

Veintitrés informantes afirmaron que los indígenas eran discriminados, lo que representa el 92\% de los que hicieron comentarios. Por lo tanto, la discriminación contra los indígenas en León se puede considerar un problema grave. Las respuestas de los encuestados se dividieron en cinco tipos diferentes de discriminación, siguiendo la clasificación de Contreras Soto (2014: 89-94): la discriminación a través de expresiones faciales, los gestos, las palabras, la risa y la violencia física.

No sólo se les hacía más difícil encontrar un empleo, también sufrían de discriminación por parte de las autoridades, quienes practicaban el fichaje racial. El informante I-3.1, por ejemplo, mencionó que fue detenido en la calle por la policía como sospechoso de un robo, mientras que otros jóvenes ni siquiera fueron abordados o interrogados. La policía lo consideró un posible ladrón, ya que llevaba sandalias y por lo tanto sospechaban que era indígena. La informante I-2.5 dijo que se les prohibía vender en el centro de la ciudad y en los mercados locales: "Antes nos quitaban la mercancía, cuando íbamos a vender en los tianguis. Los reglamentos, Dirección de Comercio del Mercado. [...] También vendíamos en el centro, pero nos quitaron, porque [dicen que] vendemos cosas chinas".

Además, los indígenas están constantemente expuestos a las miradas críticas y despectivas por su forma de hablar. La informante I-2.1 mencionó: "Luego nos ven hablando así entre nosotros mismos. Mal piensan las personas". La informante I-2.6 dijo: "Cuando llegué [a León], los vecinos me veían así, raro, poca cosa, porque sabían que soy de un pueblo; y al principio me sentía feo, pero después valoré y presumí hablando en mi idioma". También la informante l-2.7 tuvo experiencias de este tipo: "Unas personas te ven de abajo por arriba, ven qué calzado traes, preguntan 'por qué traes esto', ‘Ponte unos zapatos!'”. Los indígenas perciben que no son parte de la sociedad, sino diferentes. La informante I-3.7 declaró: "Porque nos ven como extraterrestres, una cosa de otro mundo". El profesor Francisco, quien enseñaba en el Centro Educativo Intercultural Nenemi, supo que algunos niños ya no querían hablar sus idiomas indígenas fuera del CDIL. Estaban entre dos lenguas y debido a las experiencias negativas que tuvieron al hablar sus lenguas maternas fuera del área protegida y conocida, no se sentían libres de hablar su idioma: "Sí, así cuando yo he platicado con algunos de mis alumnos, a algunos pues sí se les hace difícil platicar, conversar fuera, allá en la calle. Porque 
precisamente sienten que, cuando la gente te empieza a mirar, es por algo. [...] Y los niños [...] se sienten mal".

La mayoría de los encuestados, entre ellos ocho niños, dijeron que habían sufrido abusos verbales. La población no indígena les decía "indígenas", "indios", "inditos" o "indias Marías"14. El informante I-1.7 ya no usaba su traje tradicional en la calle para evitar ser reconocida como indígena y así evitar la discriminación. El informante I-2.3 dijo: "[A] nuestras mamás, o señoras grandes que usaban antes vestidos, [les decían] 'las Marías, las Marías' [por] su forma de vestir”. La informante I-3.2 mencionó la intimidación por hablar en su idioma: "Hay personas que no nos conocen y nos insultan por lo que hablamos".

Algunos declararon que tenían que aguantar burlas, algo mencionado por los informantes I-1.6, I-2.3 y I-3.6, entre otros. En la declaración de la informante I-3.6 se puede observar el temor de sufrir un asalto violento debido al uso de su idioma materno fuera del hogar:

Hay gente, como que te burla. Mestizos, pues, en la calle. Ante vamos a ofrecer la mercancía a la gente [...] y dice la gente: "Mira, allá van las indias mayas, las marías", porque ya ves que los gentes mestizos dice. Y mi tía no tenía miedo y cuando nos decía la gente así, y dice mi tía: "Nosotros estamos trabajando, no robamos... somos lo que somos, pero somos hijos de dios". Así nos burlaba. [...] Mi mamá cuando encontraba la gente del pueblo y decía a nosotros: “'Ya vendieron?”, y le decía: "Ya no hay nada de venta. No más la gente, donde pasamos la gente, se ríe de nosotros porque hablamos otra lengua. Pero no le hacemos caso", y decía a mi mamá: "Ya no habla". Tenía miedo que nos iba a agarrar la gente.

Varios encuestados dijeron haber sido atacados. Al informante I-2.3 le habían agredido físicamente: "Hay unas personas que no tienen estudios aquí, que son drogadictos [...] pues empiezan a golpear a las personas y dicen 'pinche indio, regrésate a tu pueblo, de donde viniste', y todo esto, y sí golpean. [...] Y personas que sufren maltratos por la misma policía”. El informante I-1.2, un niño, dijo que había sido insultado y apedreado por hablar su lengua materna en las inmediaciones del CDIL:

[Unos] niños nos andaban diciendo que éramos unos indios y que... éramos indígenas... que éramos de antes... y que ya no éramos como ellos y ellos sí sabían bien hablar el español. Y luego nos aventaron piedras y nos venimos corriendo para la casa... y de allí le dijimos a mi mamá, y mi mamá le fue a reclamar a la señora, y la señora también le dijo que era una india.

14. Este nombre tiene su origen en el personaje ficticio de la India María, interpretada por la comediante María Elena Velasco en películas y televisión a partir del largometraje Tonta, tonta, pero no tanto (1972). Ella encarna los estereotipos negativos de los pueblos indígenas como el analfabetismo, el desconocimiento de la cultura dominante y el hablar un castellano etnolectal, es decir, con acento (Pineda-Dawe, 2012). 
Al parecer, los estereotipos y la discriminación hacia los indígenas en la sociedad mexicana surgen de las actitudes negativas que los no indígenas tienen hacia los pueblos originarios. Por esto se producen los actos de discriminación descritos aquí. La experiencia de esta hostilidad puede producir vergüenza en los indígenas y, como resultado, puede resultar en la represión de características externas de identidad, como hablar una lengua indígena. Puede motivar que estas minorías lingüísticas, quienes viven en el espacio intermedio que hemos estado analizando, decidan dejar sus lenguas para ser asimilados en la sociedad mayoritaria y así ser respetados.

\subsection{Cómo las experiencias influyen en las actitudes acerca de los idiomas y su uso}

Los entrevistados mencionaron que por un lado amaban a sus culturas y que en sus comunidades tenían actitudes positivas hacia sus lenguas maternas, porque eran parte de su cultura, de su forma de ser. Pero las actitudes negativas de los no indígenas provocaban sentimientos de vergüenza en diez de los entrevistados. Estas actitudes negativas externas pueden cambiar sus actitudes y provocar el abandono de sus lenguas maternas. A partir de los comentarios de varios encuestados, se puede observar que el desplazamiento de sus lenguas está motivado, de alguna manera, por la discriminación recibida por parte de los no indígenas al escucharlos hablar en su lengua materna.

El informante I-2.4 declaró: "Antes sí había mucha discriminación. Por eso mucha gente ya no habla alguna lengua aunque lo sepa". También el informante I-1.10 dijo que le daba vergüenza hablar el otomí en la calle y que por eso ya no lo hacía. La informante I-3.6, quien fue llamada 'India María' y fue burlada por hablar su lengua materna, ya quería dejar de hablarla y por eso dijo a su madre: "Ya no habla". También la informante I-1.11 consideraba dejar su idioma y compartió sus experiencias negativas:

Un día fui a vender rosas, de 12 años, y una niña se acercó a mí, y [...] me agarró y me jaló el cabello, y [...] ella me dijo "india". Y otro día pasé en la misma calle [...] y ya venía, y luego de allí, me agarró la mano, y me agarró del cabello, y me apretó, y luego me puso una bolita de chicle, y llegué a la casa, y [...] mi mamá me dijo: "Déjala, es loca". Yo dije a mi mamá, "Mejor ya no hablo el náhuatl”, pero mi mamá me dijo que mejor lo hablara, que no la hiciera caso; y lo sigo hablando.

En este caso, la madre animó a su hija a seguir hablando náhuatl. También una señora tarasca deseaba que su hijo no dejara de hablar su idioma: "Depende de los niños, si quiere seguir hablando. Hay personas, les da pena de hablar el idioma, y lo dejan de hablar. Yo sí le digo a mi niño que no deje de hablar en purépecha".15

15. 'Purépecha' es una de las autodenominaciones del idioma tarasco (Instituto Nacional de Lenguas Indígenas, 2009: 289, 324). 


\section{Conclusiones}

Se ha mostrado que en la ciudad mexicana de León los indígenas son vulnerables, marginalizados y padecen de discriminación. Viven no sólo entre dos culturas, sino también entre dos lenguas. El uso de un idioma indígena puede causar la estigmatización de sus hablantes y esto ha sido un factor que desmotiva el uso de sus lenguas maternas. Por lo tanto, el menosprecio hacia los indígenas por la población no indígena está incidiendo en el desplazamiento de las lenguas maternas de los pueblos originarios. En León la fundación del Centro de Desarrollo Indígena Loyola, así como el Centro Educativo Intercultural Nenemi, son esfuerzos notables de la sociedad civil para apoyar a los indígenas. Sin embargo, para contrarrestar la tendencia del abandono de sus rasgos culturales por los indígenas, no es suficiente el aprecio y el apoyo de estas organizaciones. Si la sociedad valorara más las culturas de los indígenas, incluyendo sus idiomas, considerándolos como capital cultural y simbólico, los indígenas sentirían menos vergüenza y más orgullo, condición necesaria para la reivindicación y la revitalización de estas lenguas en un contexto urbano como el de León. ${ }^{16}$ Queda mucho camino que andar para que los pueblos originarios alcancen la situación de respeto y dignidad que merecen, después de cinco siglos de resistencia ante las presiones colonizadoras.

16. La Declaración Universal de Derechos Lingüísticos, proclamada en Barcelona en 1996, expresa el consenso entre hablantes de lenguas minoritarias, escritores, organizaciones no gubernamentales, investigadores y activistas, con el apoyo moral y técnico de la UNESCO, sobre lo que deben ser los derechos lingüísticos. Fue un punto de partida relevante en este sentido e influyó en la legislación de varios países, incluyendo México (Comité de Seguimiento, 1998). 


\section{Referencias bibliográficas}

Abelló Contesse, Christián (2010), "El bilingüismo familiar no convencional y el papel dependiente del enfoque UPUL”, en Christián Abelló Contesse, Christoph Ehlers y Lucía Quintana Hernández (eds.), Escenarios bilingües: el contacto de lenguas en el individuo y la sociedad, Berna, Peter Lang, p. 171-209.

Appel, René y Pieter Muysken (2005), Language contact and bilingualism, Ámsterdam, Amsterdam University Press.

Balam, Benito (José Arturo Fuentes Creollo) (2003), La intervención social con indígenas migrantes: experiencias, cuestionamientos y propuestas, León, Universidad Iberoamericana León.

Bollée, Annegret e Ingrid Neumann-Holzschuh (2009), Spanische Sprachgeschichte - Uni-Wissen Spanisch, Stuttgart, Klett.

Bonfil Batalla, Guillermo (1990), México profundo: una civilización negada, Ciudad de México, Consejo Nacional para la Cultura y las Artes/Grijalbo.

Centro de Desarrollo Indígena Loyola (2014), Censo de la población que habita en el Centro de Desarrollo Indígena Loyola A. C. [Documento inédito.]

Comisión Nacional para el Desarrollo de los Pueblos Indígenas (2010a), Indicadores sociodemográficos de la población total y la población indígena, disponible en http://www.cdi.gob.mx/cedulas/2010/GUAN/11020-10.pdf. [Sitio consultado el 14 de enero de 2015.]

Comisión Nacional para el Desarrollo de los Pueblos Indígenas (2010b), Memoria de la consulta sobre migración de la población indígena, disponible en https://www.gob.mx/cms/uploads/attachment/file/37020/consulta_migracion_poblacion_indigena.pdf. [Sitio consultado el 14 de enero de 2015.]

Comité de Seguimiento (1998), Declaración Universal de Derechos Lingüísticos, disponible en https:// www.pencatala.cat/wp-content/uploads/2016/02/dlr_espanyol.pdf. [Sitio consultado el 14 de enero de 2015.]

Contreras Soto, Ricardo (2014), "Discriminación a los grupos indígenas: acercamiento al planteamiento general", en David Charles Wright-Carr y Daniel Vega Macías (eds.), Los pueblos originarios en el estado de Guanajuato, Ciudad de México/Guanajuato, Pearson Educación de México/ Universidad de Guanajuato, p. 77-102.

Geiger, Hanna Lene (2010): Maestra, ¿Qué es un indígena?: Sprechereinstellungen in einer mehrsprachigen Gemeinschaft in Oaxaca, Mexiko. [Documento inédito.]

Gerhard, Peter (1993), A guide to the historical geography of New Spain: revised edition, Norman / Londres, University of Oklahoma Press.

Huerta Arellano, Cathia, Ana Patricia Beltrán Soria y Mariana Aguilar Doncel (2012), Situación de los indígenas en León, Guanajuato. [Documento inédito.] 
Instituto Nacional de Estadística y Geografía (2010), Censo de población y vivienda 2010, disponible en <https://www.inegi.org.mx/programas/ccpv/2010/>. [Sitio consultado el 13 de enero de 2015.]

Instituto Nacional de Lenguas Indígenas (2009), Catálogo de las lenguas indígenas nacionales: variantes lingüísticas de México con sus autodenominaciones y referencias geoestadísticas, ed. digital, Ciudad de México, Instituto Nacional de Lenguas Indígenas, disponible en https://www.inali. gob.mx/clin-inali/. [Sitio consultado el 26 de septiembre de 2020.]

Irala Burgos, Fernando (2008), La SEDESOL y el gobierno de Guanajuato firmaron el convenio "Contigo vamos por más oportunidades", disponible en https://www.prospera.gob.mx/Portal/wb/Web/ la_sedesol_y_el_gobierno_de_guanajuato_firmaron_el. [Sitio consultado el 3 de marzo de 2015.]

Jasso Martínez, Ivy Jacaranda (2011), "Vulnerabilidad y población indígena en León, Guanajuato", Ide@s CONCYTEG, vol. 6, n 75, p. 1113-1136, disponible en http://convivejoven.semsys.itesi. edu.mx/cargas/Articulos/VULNERABILIDAD\%20Y\%20POBLACI\%C3\%93N\%20INDIGENA.pdf. [Sitio consultado el 28 de septiembre de 2020.]

Jasso Martínez, Ivy Jacaranda (2014), "Procesos de discriminación y exclusión entre mujeres indígenas migrantes en León", en David Charles Wright-Carr y Daniel Vega Macías (eds.), Los pueblos originarios en el estado de Guanajuato, Ciudad de México/Guanajuato, Pearson Educación de México/Universidad de Guanajuato, p. 103-129.

Jasso Martínez, Ivy Jacaranda (2015), "Retos y exigencias en los procesos de socialización: juventud, etnicidad y migración en León, Guanajuato", Ra Ximhai, vol. 11, n² 2, p. 93-116, disponible en https://www.redalyc.org/pdf/461/46143101006.pdf. [Sitio consultado el 28 de septiembre de 2020.]

Kocianová, Maria (2005), Metasprachliche Fähigkeiten zweisprachiger Kinder: Zum Zusammenhang von sprachlicher und metasprachlicher Leistungsfähigkeitund die damit einhergehenden Implikationen für eine adäquate Förderung der Russisch-Deutsch sprechenden Kinder im Grundschulalter, tesis de doctorado, Múnich, Universidad de Múnich.

Marañón Lazcano, Felipe de Jesús y Carlos Muñiz (2012), "Estereotipos mediáticos de los indígenas: análisis de las representaciones en programas de ficción y entretenimiento de televisoras en Nuevo León", Razón y Palabra, n 80, disponible en http://www.razonypalabra.org.mx/N/N80/ V80/20_MaranonMuniz_V80.pdf. [Sitio consultado el 4 de febrero de 2015.]

Martínez de la Rosa, Alejandro, Ivy Jasso Martínez y David Charles Wright Carr (2016), "Guerreros chichimecas: la reivindicación del indio salvaje en las danzas de Conquista”, Relaciones, Estudios de Historia y Sociedad, vol. 37, n 145, p. 251-278, disponible en http://www.revistarelaciones.com/ index.php/relaciones/article/view/30. [Sitio consultado el 3 de noviembre de 2020.]

Ministerio de Educación y Formación Profesional (2019), La enseñanza de lenguas cooficiales, regionales o minoritarias en las administraciones educativas, disponible en https://www.educacionyfp.gob.es/dam/jcr:e86dbc5b-8fc5-45c1-a5be-53e89662d77c/tabla-1.pdf . [Sitio consultado el 28 de septiembre de 2020.] 
Montemayor, Carlos (2000), Los pueblos indios de México hoy, Ciudad de México, Planeta.

Pfeiler, Barbara (1988), "El uso de dos lenguas en contacto", Estudios de Cultura Maya, nº 17, p. 423444.

Pineda-Dawe, Mariana (2012), Estereotipo, frontera y género: una lectura negociada de tres cintas de la India María, tesis de doctorado, Montreal, Université de Montréal, disponible en https://papyrus.bib.umontreal.ca/xmlui/bitstream/handle/1866/9189/Pineda_Dawe_Mariana_2012_memoire.pdf. [Sitio consultado el 28 de septiembre de 2020.]

Przyborski, Aglajay Monika Wohlrab-Sahr (2014), Qualitative Sozialforschung: ein Arbeitsbuch, Múnich, Oldenbourg Verlag.

Real Academia Española (1925), Diccionario de la lengua española, Madrid, Real Academia Española, disponible en https://www.rae.es/sites/default/files/Prologo_de_la_edicion_de_1925.pdf. [Sitio consultado el 28 de septiembre de 2020.]

Schnuchel, Sophia (2016a), "(Des-)conocimientos de indígenas acerca de sus derechos lingüísticos", Epikeia: Revista del Departamento de Ciencias Sociales y Humanidades, $n^{\circ} 30$, disponible en http://epikeia.leon.uia.mx/numeros/30/des-conocimientos-de-indigenas-acerca-de-sus-derechos-linguisticos.php. [Sitio consultado el 19 de septiembre de 2020.]

Schnuchel, Sophia (2016b), "Ya no quieren hablar sus lenguas". Der Kontakt des Spanischen mit indigenen Sprachen in León, Mexiko - Eine soziolinguistische Studie, Erlangen, Sektion Iberoamerika, Zentralinstitut für Regionenforschung, Universität-Nürnberg, disponible en https://opus.bibliothek.uni-augsburg.de/opus4/frontdoor/index/index/docld/. [Sitio consultado el 19 de septiembre de 2020.]

Schnuchel, Sophia (2017), "Circunstancias vitales de gente indígena en un contexto urbano - el caso de León de los Aldama”, Acta Universitaria: Multidisciplinary Scientific Journal, vol. 27, n 4, p. 6982, disponible en http://www.actauniversitaria.ugto.mx/index.php/acta/article/view/1288/0. [Sitio consultado el 19 de septiembre de 2020.]

Schnuchel, Sophia (2018), "Bilingüismo de indígenas migrantes y desplazamiento de idiomas autóctonos en León, Guanajuato", Relaciones: Estudios de Historia y Sociedad, vol. 39, n 155, p. $167-$ 207, disponible en http://www.revistarelaciones.com/index.php/relaciones/article/view/ REHS15507. [Sitio consultado el 19 de septiembre de 2020.]

Sommerhoff, Gerhard y Christian Weber (1999), Mexiko - Geographie, Geschichte, Wirtschaft, Politik, Darmstadt, Wissenschaftliche Buchgesellschaft.

Téllez, Ivonne (2014), Datos primavera 2014 completo. [Documento inédito.]

Terborg, Roland y Laura García Landa (eds.) (2011), Muerte y vitalidad de las lenguas indígenas y las presiones sobre sus hablantes, Ciudad de México, Centro de Enseñanza de Lenguas Extranjeras, Universidad Nacional Autónoma de México, disponible en https://ela.enallt.unam.mx/index. php/ela/article/view/493. [Sitio consultado el 3 de noviembre de 2020.] 
Tutino, John (2011), Making a New World: founding capitalism in the Bajio and Spanish North America, Durham/Londres, Duke University Press.

Weinel, Meike (2011): Bedrohte Sprachen zwischen Sprachtod und Spracherhalt am Beispiel des Palenquero: Sprechereinstellungen und Sprachverhalten der jungen Generation in Barranquilla. [Documento inédito.]

White, Richard (2011), The middle ground: Indians, empires, and republics in the Great Lakes region, 1650-1815, twentieth anniversary edition, Cambridge, Cambridge University Press. [1ª ed., 1991.]

Wolf, Eric R. (2001). Pathways of power: building an anthropology of the modern world, Berkeley/Los Ángeles/Londres, University of California Press.

Wright-Carr, David Charles (2014), "La prehistoria e historia temprana de los pueblos originarios del Baji”, en David Charles Wright-Carr y Daniel Vega Macías (eds.), Los pueblos originarios en el estado de Guanajuato, Ciudad de México/Guanajuato, Pearson Educación de México/Universidad de Guanajuato, p. 1-40.

Wright-Carr, David Charles (2016), "Prólogo", en Sophia Schnuchel, "Ya no quieren hablar sus lenguas", der Kontakt des Spanischen mitindigenen Sprachen in León, Mexiko - Eine soziolinguistische Studie, Erlangen, Sektion Iberoamerika, Zentralinstitut für Regionenforschung, Universität-Nürnberg, disponible en https://opus.bibliothek.uni-augsburg.de/opus4/frontdoor/index/index/docld/. [Sitio consultado el 19 de septiembre de 2020.]

Wright-Carr, David Charles (2017), "Signs of resistance: iconography and semasiography in Otomi architectural decoration and manuscripts of the early colonial period", Visible Language, vol. 51, $n^{\circ} 1$, p. 58-87, disponible en http://visiblelanguage.herokuapp.com/issue/242/article/1602. [Sitio consultado el 3 de noviembre de 2020.] 


\section{Apéndice: Relación de las personas entrevistadas}

\begin{tabular}{|c|c|c|c|c|c|}
\hline & Sexo & $\begin{array}{l}\text { Lugar de origen (población y } \\
\text { estado) }\end{array}$ & $\begin{array}{c}\text { Edad } \\
\text { en } \\
\text { años }\end{array}$ & $\begin{array}{l}\text { Último nivel } \\
\text { de estudios }\end{array}$ & $\begin{array}{l}\text { Agrupación } \\
\text { lingüística }\end{array}$ \\
\hline \multicolumn{6}{|c|}{ Grupo I-1 (8-13 años) } \\
\hline $\mid-1.1$ & femenino & Acultzingo, Veracruz & 8 & $3^{\circ}$ primaria & náhuatl \\
\hline $1-1.2$ & masculino & $\begin{array}{c}\text { Puebla, Puebla y Comonfort, } \\
\text { Guanajuato }\end{array}$ & 8 & $3^{\circ}$ primaria & $\begin{array}{c}\text { otomí y totona- } \\
\text { co }\end{array}$ \\
\hline $\mid-1.3$ & femenino & $\begin{array}{c}\text { Santa María Cahuañaña, Gue- } \\
\text { rrero }\end{array}$ & 11 & $3^{\circ}$ primaria & mixteco \\
\hline $\mid-1.4$ & femenino & $\begin{array}{c}\text { Santiago Mexquititlán, Queré- } \\
\text { taro }\end{array}$ & 9 & $4^{\circ}$ primaria & otomí \\
\hline $\mid-1.5$ & masculino & $\begin{array}{c}\text { León, Guanajuato (familia de } \\
\text { Loma Linda, Veracruz) }\end{array}$ & 10 & $4^{\circ}$ primaria & náhuatl \\
\hline ।-1.6 & masculino & $\begin{array}{c}\text { Guadalajara, Jalisco e Ichán, } \\
\text { Michoacán }\end{array}$ & 10 & $4^{\circ}$ primaria & tarasco \\
\hline $1-1.7$ & masculino & Loma Linda, Veracruz & 12 & $5^{\circ}$ primaria & náhuatl \\
\hline $1-1.8$ & masculino & Macvilhó, Chiapas & 11 & $5^{\circ}$ primaria & tsotsil \\
\hline $1-1.9$ & femenino & Orizaba, Veracruz & 13 & $5^{\circ}$ primaria & náhuatl \\
\hline $1-1.10$ & masculino & $\begin{array}{c}\text { Santiago Mexquititlán, Queré- } \\
\text { taro }\end{array}$ & 11 & $5^{\circ}$ primaria & otomí \\
\hline $\mid-1.11$ & femenino & Loma Linda, Veracruz & 12 & $6^{\circ}$ primaria & náhuatl \\
\hline $\mid-1.12$ & masculino & Ichán, Michoacán & 12 & $6^{\circ}$ primaria & tarasco \\
\hline \multicolumn{6}{|c|}{ Grupo I-2 (14-28 años) } \\
\hline $\mid-2.1$ & femenino & Loma Linda, Veracruz & 14 & $2^{\circ}$ secundaria & náhuatl \\
\hline $1-2.2$ & masculino & $\begin{array}{c}\text { Santa María Cahuañaña, } \\
\text { Guerrero }\end{array}$ & 18 & sin estudios & mixteco \\
\hline $1-2.3$ & masculino & Silacayoápam, Oaxaca & 21 & universidad & mixteco \\
\hline $1-2.4$ & masculino & Ichán, Michoacán & 25 & $6^{\circ}$ primaria & tarasco \\
\hline $1-2.5$ & femenino & Ichán, Michoacán & 26 & $3^{\circ}$ secundaria & tarasco \\
\hline $1-2.6$ & femenino & Comachuén, Michoacán & 27 & $3^{\circ}$ secundaria & tarasco \\
\hline $1-2.7$ & femenino & Loma Linda, Veracruz & 28 & $6^{\circ}$ primaria & náhuatl \\
\hline \multicolumn{6}{|c|}{ Grupo I-3 (30-53 años) } \\
\hline $1-3.1$ & masculino & Tepecuitlapa, Veracruz & 30 & $3^{\circ}$ secundaria & náhuatl \\
\hline $1-3.2$ & femenino & Ichán, Michoacán & 31 & $6^{\circ}$ primaria & tarasco \\
\hline $1-3.3$ & femenino & Loma Linda, Veracruz & $45-50$ & sin estudios & náhuatl \\
\hline |-3.4 & masculino & Silacayoápam, Oaxaca & 47 & $6^{\circ}$ primaria & mixteco \\
\hline
\end{tabular}




\begin{tabular}{|c|c|c|c|c|c|}
\hline I-3.5 & masculino & Loma Linda, Veracruz & 49 & $2^{\circ}$ primaria & náhuatl \\
\hline I-3.6 & femenino & Santana, México & 49 & $2^{\circ}$ primaria & mazahua \\
\hline I-3.7 & femenino & Ichán, Michoacán & 52 & $6^{\circ}$ primaria & tarasco \\
\hline I-3.8 & femenino & Ichán, Michoacán & 53 & sin estudios & tarasco \\
\hline
\end{tabular}




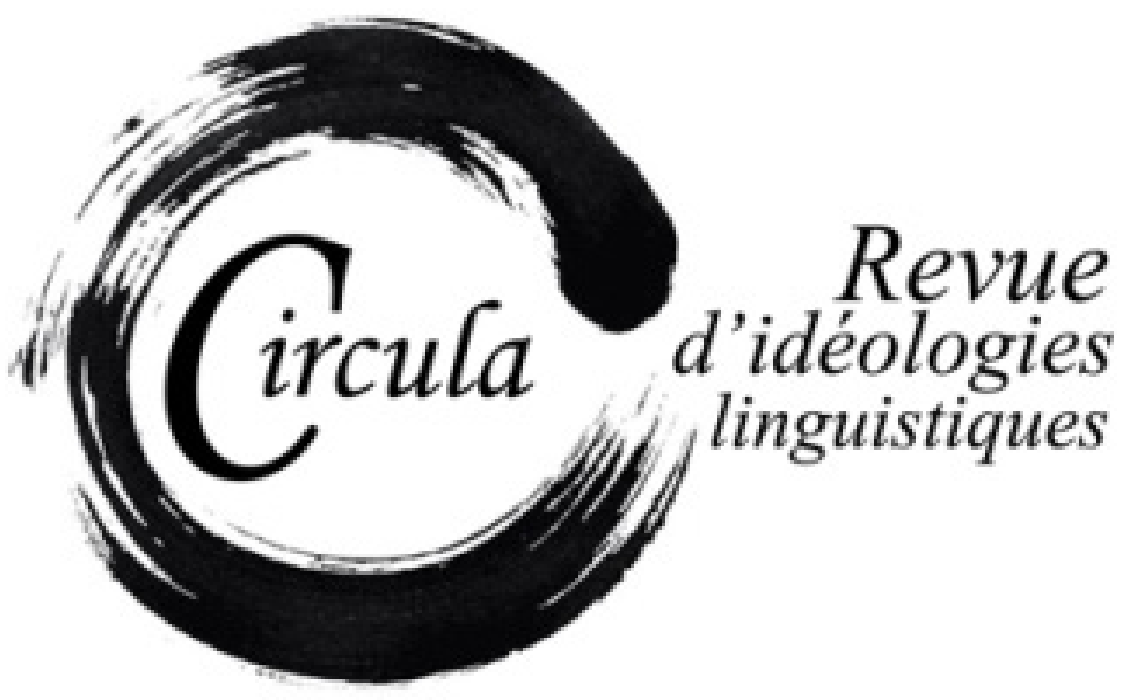

TITRE: DISSOCIER ALLOPHONIE ET VULNÉRABILITÉ : DE LA NÉCESSITÉ D'INTERROGER LES CATÉGORIES

Auteur: Maxime Alais, Université de Tours/UClouvain, Silvia LuCChini, UClouvain, Salima El

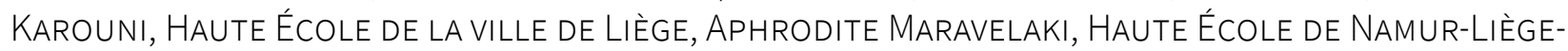
LUXEMBOURG, ÉLODIE OGER, UCLOUVAIN

Revue: Circula, NumÉro 12 : LA VULNÉRABILITÉ LINGUISTIQUE

DiRECTRICE: CLAUDIA TORRES CASTILLO

PAges: $87-106$

ISSN: 2369-6761

URI: HTTP://HDL.HANDLE.NET/11143/18444

DOI: HTTPS://DOI.ORG/10.17118/11143/18444 


\title{
Dissocier allophonie et vulnérabilité : de la nécessité d'interroger les catégories
}

\author{
Maxime Alais, Université de Tours/UCLouvain \\ maxime.alais@univ-tours.fr
}

Silvia Lucchini, UCLouvain silvia.lucchini@uclouvain.be

Salima El Karouni, Haute École de la ville de Liège salima.elkarouni @ hel.be

\begin{abstract}
Aphrodite Maravelaki, Haute École de Namur-Liège-Luxembourg afroditi.maravelaki@henallux.be
\end{abstract}

Élodie Oger, UCLouvain

elodie.oger@uclouvain.be

Résumé : Cet article s’interroge sur l'établissement des catégories d'élèves définies par les institutions éducatives belges francophones (Fédération Wallonie-Bruxelles, FWB) concernant la scolarisation des primoarrivants. Plus précisément, il s’agit de se demander si le lien établi par ces institutions ne crée pas un amalgame entre les élèves primoarrivants et ceux dits vulnérables, via un critère de non-maitrise supposée de la langue d'enseignement, ou du français langue d'apprentissage (FLA). Si les catégories définies par les autorités éducatives et les liens qu'elles établissent entre les élèves s'inscrivent dans un objectif global et louable d'aide à la scolarisation des élèves primoarrivants, nous considérons que ces catégories et liens qu'elles établissent viennent renforcer le regard négatif habituellement porté sur ce public scolaire et ne reconnaît pas suffisamment la diversité du public scolaire considéré dans son ensemble. Nous plaidons donc pour une dissociation entre l'allophonie et la vulnérabilité.

Mots-clés : Belgique francophone ; primoarrivants ; allophones ; regard déficitaire ; FLA 
Summary: This contribution questions the creation of categories of students as established by the French-speaking Belgian education authorities (Fédération Wallonie-Bruxelles, FWB) concerning the schooling of newcomers. More precisely, we are asking if the link established by these authorities doesn't create an amalgam between newcomer students and these students called vulnerable; they are supposedly linked together by the fact that they do not master the language of instruction, or French as the language of learning (français langue d'apprentissage, FLA). While these categories and the links they create between the students are based on a global and laudable objective of helping the schooling of newcomer students, we think that these categories and the links they create reinforce the negative perception people often have of this public and does not sufficiently recognise the diversity of the schooling public as a whole. We therefore argue for a dissociation between "allophones" and vulnerable students.

Keywords: French-speaking Belgium; newcomers; allophones; French as the language of learning 


\section{Introduction}

Depuis les années 1990, les pouvoirs publics belges francophones s'attachent à favoriser l'enseignement du français auprès des publics non francophones ou issus de l'immigration. Parmi les actions qui se sont multipliées en faveur de l'enseignement primaire ou secondaire, citons les cours de langues et de cultures d'origine (1996), présentés comme un tremplin pour une meilleure acquisition de la langue de scolarisation, les classes passerelles (2001) remplacées par les dispositifs d'accueil et de scolarisation des élèves primoarrivants (ou DASPA, 2012) ou, plus récents, les dispositifs de français langue d'apprentissage (FLA, 2019). Le développement de cours de FLE dans les hautes écoles (2001) et à l'université et de parcours d'intégration pour adultes primoarrivants non européens (2012) participent du même esprit. Pour une présentation de ces différentes mesures, on peut se référer à un article récemment publié par notre groupe (ALIA, 2018). Dans ce même article, nous défendions l'idée que si ces différentes mesures poursuivaient un objectif louable - favoriser l'intégration et la réussite scolaire/sociale des personnes immigrées ou issues de l'immigration -, elles avaient, dans certains cas, contribué à renforcer les processus de ségrégation à l'œuvre dans le système éducatif belge, qui, comme nous le savons depuis les enquêtes PISA, est l'un des plus inéquitables. Ces effets délétères ont du reste été cernés par de nombreux chercheurs en sciences de l'éducation qui ont porté leur attention plus généralement sur un public défavorisé et sur les mesures de discrimination positive (Merle, 2012). Le principal est probablement l'inégalité d'acquis, donnant un accès différencié aux diplômes et, par la suite, aux ressources socioéconomiques et sociétales.

\section{L’association hétéroattribuée entre allophonie et vulnérabilité}

On discerne presque toujours, à l'origine des mesures mentionnées, un espace de représentations au sein duquel les personnes issues de l'immigration sont perçues comme étrangères, non francophones ou parlant un français incorrect :

- Étranger. Même lorsqu'elles ont la nationalité belge, même après deux générations de permanence dans le pays de résidence. Nous avons montré dans une précédente étude qui portait sur la relation au français et aux autres langues de personnes issues de l'immigration de première, deuxième et troisième générations (Lucchini et al., 2012) que ce qui les qualifie d'étrangers, indépendamment de la nationalité, se rattache à des marques ethniques visibles. Un certain nombre d'informateurs interrogés dans la recherche en question estiment que l'apparence physique en particulier identifie la personne comme étrangère et est génératrice d'exclusion. À cause de la couleur d'abord, si celle-ci s'écarte d'un supposé phénotype prototypique local, caractérisé par « le blanc », «le blond » et « le bleu ». À ce propos, l'effet discriminant de la visibilité de la différence a depuis longtemps été mis en évidence (Beauchemin et al., 2010) et le « délit de sale gueule » est depuis longtemps dénoncé comme étant à l'origine de contrôles exagérés et abusifs. 
La caractéristique « étranger » est ensuite attribuée à partir du nom. En particulier du prénom. Une très intéressante étude de Prikhodkine et al. (2008) a montré que le prénom exolingue, parce qu'il traduit le choix explicite des parents, inscrit les individus dans la différence ethnique et est source de discrimination au travail en Suisse romande.

- Non francophone ou parlant un mauvais français, caractéristiques activées par la catégorie « étranger». Parfois l'attribution correspond à des faits. On peut en effet arriver dans le pays sans connaitre la langue qui y est parlée. Ou les enfants peuvent débuter leur scolarité en ayant entendu exclusivement une autre langue que le français dans leur milieu familial. Concernant la pratique d'un français non normé, on peut avoir appris le français en milieu scolaire exolingue, l'avoir peu pratiqué avant l'arrivée dans le pays d'accueil, sans l'avoir acquis de façon comparable à un natif. D'autant plus si le faible niveau de scolarité dans le pays d'origine, ou une absence de scolarité, n'a pas facilité l'analyse des différences et des ressemblances entre le français et la langue initialement parlée. Comme Klein (1989) le souligne, ce qui permet l'évolution d'une langue apprise en milieu naturel est la comparaison constante par le non-natif entre la langue qu'il produit et la production des natifs. L'acquisition s'arrête quand le non-natif n'est plus en mesure de percevoir des différences. Pour percevoir les différences, une analyse des deux langues est nécessaire. Et ce qui aide à le faire, ce sont les analyses explicites et parfois fastidieuses qu'on a effectuées au cours de la scolarité. Ces dernières ont en réalité contribué à former une sorte de grammaire intérieure de référence sur laquelle on se base pour comparer ${ }^{1}$.

Parfois l'association entre l'allophonie et la non-connaissance du français ou l'acquisition non normée du français se justifie lorsque les enfants ont grandi dans des contextes d'hybridation linguistique dans des quartiers populaires ethniquement homogènes, où la seule personne qui parle un français standard est finalement l'enseignant. Le phénomène est le même que celui qui a donné lieu à des pidgins ou à des créoles, où les deux langues en contact en ont produit une troisième possédant des caractéristiques des deux² (Lucchini, 2005). C'est donc cette langue partiellement composée de français et partiellement d'autres langues que les enfants ont entendue depuis leur plus jeune enfance et qu'ils ont apprise avant d'entrer à l'école, surtout si les contacts avec des natifs ont été réduits, ce qui est souvent le cas dans les quartiers ethniquement homogènes.

1. L'exemple de l'usage correct du participe présent italien est éclairant. Dans sa forme, le participe présent italien est différent du gérondif, contrairement au français, langue dans laquelle il est facile de les confondre. Les étudiants francophones utilisent en général le gérondif en italien à la place du participe présent car en français ils n’ont souvent pas appris à analyser correctement les deux et à les distinguer. Ils commettent donc l'erreur en italien à cause de cette défaillance d'analyse en français.

2. La différence entre les pidgins et la langue hybride parfois parlée dans les familles est que cette dernière est instable et fluctuante, sans doute à cause à la présence concomitante et constante des langues sources. 
Toutefois, la représentation des individus immigrés ou issus de l'immigration en tant qu'étrangers et des étrangers en tant que non francophones ou parlant un mauvais français peut être abusive et est le résultat d'hétéroattributions indûment généralisatrices. La confrontation avec une réalité contraire à la représentation ne la corrige pas. Le plus bel exemple est le dialogue entre l'enfant Girolamo Santocono, qui vit dans la Rue des Italiens, et Monsieur Léon, le marchand de fruit et légumes du coin :

De toute façon, comme il n'est resté presque exclusivement que les Italiens dans la rue, il a bien dû s'habituer. Et, dans un élan condescendant digne d'un touriste européen mettant la djellaba au cours de vacances en Tunisie, monsieur Léon a même appris notre langue. En fait, dans sa tête, il suffisait de mettre un « $i$ » ou un « a » au bout de chaque mot pour qu'à coup sûr tu parles le macaroni. (...)

Je me souviens qu'un jour, j'ai voulu tester sa bonne foi. J'ai rassemblé toutes les connaissances du bon parler français que j'avais acquises à l'école et je suis allé acheter des pommes de terre chez lui. Après son traditionnel « bon giorni » et mon très courtois « bonjour monsieur Léon », il m’a demandé:

- Tu volair queq'chosa, mon petito bambino?

- Oui, j'aimerais un sac de dix kilos de pommes de terre, répondis-je. Mais pas trop grosses car c'est pour cuire au four.

- Mucho bene! Mais pommas de terras novelas ou vieillas?

Dans son regard, je ne voyais aucune moquerie et je remarquais même qu'il faisait des efforts pour me comprendre. Tel un instituteur, il tendait l'oreille en avant à chacune de mes répliques. Je prends plaisir au jeu et je continue en exagérant.

- Oh, mon Dieu ! Mère ne m’a point donné de directives à ce sujet. Donnez m'en des plus récentes!

Son front s'est crispé brusquement. Manifestement, il ne comprenait plus.

- Quoi, toi volair novelas?

- Oui, exactement.

- Ah bon, parce que toi pas bene explicato, hein!

Il m’a servi ses meilleures pommes de terre et je suis reparti rassuré une bonne fois pour toutes. L'idée qu'un étranger puisse parler le français mieux que lui ne le traversait même pas et naturellement il a continué à me parler ainsi tout le temps. (Santocomo, 1987 : 126-127)

L'origine d'une telle surgénéralisation étranger-mauvais locuteur du français est à chercher, selon l'auteur de l'extrait, dans le sentiment éprouvé par la population locale de non-reconnaissance de la légitimité des immigrés italiens dans le quartier; un sentiment de perte de l'identité, de destruction de l'habitat et des relations sociales. Groupe importé contre personnes locales isolées, groupe qui impose sa loi et sa manière de vivre à des locaux qui n'ont finalement rien demandé et qui se sentent 
envahis. Identité collective contre identité individuelle, apparemment non conciliables. La puissance des hétéroattributions est à la mesure du sentiment d'expropriation vécue par les locaux, une façon de mettre à distance, d'empêcher ce qu'ils ont pu vivre comme une colonisation illégitime et définitive.

Ces catégories d'étranger, de non francophone ou de « parlant un mauvais français » ont été également hétéroattribuées par les institutions scolaires, qui sont en fin de compte tributaires des hommes qui les créent et de leurs représentations. Dans une publication de la Fédération WallonieBruxelles (l'instance qui administre l'enseignement francophone belge) parue en 2014 et destinée aux acteurs de l'éducation, la catégorie de non francophone disparait au profit de celle d' « allophone » (quelqu'un qui parle une langue autre que le français) et la catégorie de locuteur de français non standard en celle de «francophone vulnérable ». Le remplacement, en Belgique francophone, de la catégorie d'élève non francophone par celle d'élève allophone semble être un calque du même remplacement opéré en France en 2012 avec la parution d'une circulaire « relative à la scolarisation des élèves allophones nouvellement arrivés ${ }^{3} »$. Or, dans le contexte scolaire aussi, l'allophonie et la vulnérabilité peuvent être réelles, mais également imaginaires et abusives, et ces catégories peuvent générer des effets négatifs, que nous détaillons dans les pages qui suivent. Les quatre recherches que nous allons présenter s'inscrivent dans un programme plus vaste portant sur l'acquisition des langues en contexte migratoire ${ }^{4}$. Chaque recherche présentée possède une méthodologie propre, dont nous décrivons les grandes lignes, les détails étant spécifiés dans les publications citées.

\section{Un lien institutionnalisé entre élèves primoarrivants et élèves vulnérables}

La Fédération Wallonie-Bruxelles a promulgué trois décrets successifs relatifs à l'accueil des élèves primoarrivants. Le premier décret (2001) instituait les «classes-passerelles » qui deviendront dans le deuxième décret (2012) les « dispositifs d'accueil et de scolarisation des élèves primoarrivants » (DASPA). Très récemment (2019), un troisième décret reconduit le DASPA - moyennant des ajustements - et le complète aussi par la création d'un dispositif d'accompagnement FLA (Français langue d'apprentissage).

3. Information disponible sur_https://www.education.gouv.fr/bo/12/Hebdo37/MENE1234231C.htm_. [Page consultée le 20 août 2020.] À ce propos, Auger mentionnait en 2010 que la manière dont on nomme l'autre est signifiante, la dénomination «nouvellement arrivé » remplaçant primoarrivant à cause de l'association de cette dernière formulation avec primo-infectieux. Aussi, « nouvellement arrivé » est à préférer à « étranger » dans la mesure où la nouvelle dénomination indique que celui qui vient d'arriver est au début d'un processus d'intégration. La question qui toutefois s'ensuit est, comme le rappelle Auger, jusque quand est-on considéré comme «nouvellement arrivé ». Signalons que la même remarque peut être faite au sujet d'autres dénominations : jusque quand est-on qualifié d'allophone? Jusqu'à quelle génération est-on considéré comme « issu de l'immigration »?

4. Groupe de recherche ALIA, Acquisition des langues et immigration. 
Le décret DASPA-FLA (FWB, 2019) définit trois catégories d'apprenants :

(1) celle dite des «élèves primoarrivants ». Il s'agit d'élèves nouvellement arrivés sur le territoire belge, réfugiés ou candidats réfugiés, pour lesquels il y a une présomption de non-maitrise du français ${ }^{5}$, ou d'élèves en provenance de pays en voie de développement qui ne connaissent pas « suffisamment la langue de l'enseignement pour s'adapter avec succès aux activités de leur classe d'âge » (p. 2) ;

(2) celle des « assimilés aux élèves primoarrivants » qui désigne tout élève de nationalité étrangère, inscrit dans une école de la FWB depuis moins d'un an et dont la maitrise du français est jugée insuffisante suite à la passation d'une évaluation diagnostique ;

(3) celle dite des «élèves FLA-Français langue d'apprentissage » (pour les enseignements maternel et primaire). Il s'agit d'élèves francophones qui ne maitrisent pas suffisamment « la langue de l'enseignement pour s'adapter avec succès aux activités de sa (leur) classe d'âge » (p. 2). Leur maitrise insuffisante du français est, comme pour les élèves « assimilés aux primoarrivants », attestée par une évaluation diagnostique.

L'objectif de ce dernier décret est de " proposer un accompagnement scolaire et pédagogique adapté aux profils d'enseignement des élèves primo arrivants, assimilés et FLA et lié aux difficultés relatives à la maitrise de la langue de l'enseignement et de la culture scolaire » (p. 3). Dans ces trois cas de figure, il s'agit donc d'apprendre aux élèves la langue française et la culture scolaire.

Concernant les enfants primoarrivants, après des décennies d'invisibilité de la différence linguistique de départ, les décrets successifs à partir de 2001 ont introduit des dispositifs censés « voir la différence » pour l'assumer dans le contexte scolaire. En ce sens, on peut les considérer comme un progrès. On peut également considérer comme un progrès l'élaboration d'outils diagnostiques même si ceux qui accompagnaient le dispositif de la rentrée 2019 sont à améliorer, sans nul doute.

Dans l'article précédent (ALIA, 2018), nous mettions cependant l'accent sur le risque de ségrégation opéré par les catégorisations scolaires, qui isolent les élèves concernés par rapport aux autres, avec le risque de différencier leurs parcours par l'activation de représentations spécifiques. Dans ce cas, comme nous l'avons déjà évoqué par ailleurs, « voir la différence » reviendrait à « faire la différence ». En contexte éducatif français, Galligani (2012 : §4-5) souligne également la différenciation marquée entre le public primoarrivant et celui dit ordinaire, et l'homogénéisation réductrice que les catégorisations (liées au déplacement géographique, à la nationalité, à la maitrise de la langue notamment) opèrent sur la population scolaire concernée. Le public primoarrivant est diversifié, tant au niveau des origines ethniques et culturelles, des langues parlées que de la scolarisation (Maravelaki, 2007 ; Oger, 2019). Il n'est pas rare de voir placer par exemple des élèves arabophones dans des classes

5. Information disponible sur http://enseignement.be/index.php?page=28252\&navi=4593\&rank_page=28252. [Page consultée le 23 août 2020]. 
d'alphabétisation sous les prétextes parfois énoncés par les acteurs éducatifs qu'ils doivent " apprendre l'alphabet de notre langue » ou "se familiariser avec l'écriture alphabétique », souvent sans (se) demander si ces élèves ont été scolarisés, ont étudié ou parlent d’autres langues (ce qui est d'ailleurs souvent le cas).

De façon analogue, la reconnaissance du français en tant que langue d'apprentissage, le FLA, comme auparavant le français langue de scolarisation (le FLSCO), pourrait être considérée comme un progrès, dans le sens où l'on « verrait » enfin l'existence d'élèves pour lesquels le registre de la langue scolaire n'est pas absorbé dans le milieu familial. L'école se doit donc de l'enseigner sans le prendre pour acquis. Dans ce cas aussi cependant, « voir la différence » pourrait se transformer en «faire la différence », alors que tous les enfants doivent s'approprier cette langue d'apprentissage, chacun selon ses besoins, qui doivent être identifiés par les enseignants. Au lieu d'étendre à tous les élèves l'enseignement de la langue scolaire, fall ait-il créer une nouvelle catégorie (le FLA ne constitue pas une nouvelle langue), le FLA, abréviation devenue un acronyme qui désigne maintenant, décrétalement, des apprenants?

Le public scolaire est divers et, hormis le cas d'apprenants débutant en français, cette reconnaissance de la diversité doit se faire au sein d'une classe hétérogène pour qu'elle ne se transforme pas en ségrégation, en parcours scolaire de relégation en particulier, ce qui est par ailleurs visé par la recherche de la mixité sociale et ethnique qui active les décideurs politiques en Belgique depuis un certain temps. Si la reconnaissance de la diversité du public scolaire peut s'inscrire dans celui, plus vaste, de la problématique de la reconnaissance à l'échelle de la société (Honneth, 1992), nous estimons que les institutions éducatives doivent encore s'attacher à la construction d'un commun réfléchi par tous et pour tous. C'est à l'intérieur de ce commun que l'altérité linguistique et culturelle se doit d'être abordée, comme une normalité et non sur la base de catégories isolantes.

Enfin, l'association dans un même décret entre l'apprenant primoarrivant, supposé non francophone ou allophone, et les francophones supposés vulnérables pose question. La liaison retenue est que tous ont une « mauvaise ou la partielle compréhension de la langue dans laquelle les enseignements sont dispensés $»^{6}$, une «non-maitrise de la langue de l'enseignement», correspondant pour le décret au niveau B1 du Cadre européen commun de référence pour les langues (CECRL). Une mention particulière doit être faite pour le cas des élèves primoarrivants francophones. Les compétences de ces élèves sont souvent les plus dévalorisées dans le milieu scolaire. Leur « niveau de maitrise » pourrait être jugé insatisfaisant pour plusieurs raisons : accent déviant de la norme, système scolaire d'origine jugé inférieur au nôtre, difficultés à s'exprimer à l'oral ou à l'écrit dans des situations stressantes ou sous pression, par exemple, en situation de test de placement. Lors d'une recherche doctorale en cours (Maxime Alais), la direction d'une école qui scolarise des élèves primoarrivants francophones déclare : «ils sont francophones, mais il faut se méfier ». Si on y ajoute des facteurs

6. Propos extraits de l'allocution de la ministre de l'Éducation M.-M. Schyns lors de la journée d'information du 26 août 2019 concernant le décret DASPA-FLA : http://www.enseignement.be/index.php?page=28254\&navi=4594\&rank_ page=28254. [Page consultée le 2 août 2020.] 
indépendants de l'école, décrits ci-dessus, comme la couleur ou le nom, l'amalgame avec un public «vulnérable » et « allophone » s'opère sans difficulté et peut compromettre la présomption d'un parcours scolaire de réussite pour les primoarrivants.

\section{Une diminution des attentes scolaires}

Les conséquences des catégorisations qui procèdent par des distinctions qui ne sont pas toujours basées sur une analyse des besoins linguistiques réels, dans l'espace commun d'une classe socialement et ethniquement hétérogène, risquent de produire des effets délétères pour les populations concernées. En particulier, les exigences des formateurs à l'égard de ces publics sont nivelées par le bas, ce qui explique en partie la construction inégale des acquis scolaires que nous évoquions plus haut.

La recherche doctorale menée par Oger (2019) visait à identifier, à décrire et à analyser les pratiques pédagogiques des enseignants œuvrant dans les DASPA de l'enseignement secondaire belge francophone. En particulier, elle s'est attachée à comprendre les pratiques enseignantes susceptibles de favoriser l'intégration des élèves primoarrivants dans l'enseignement ordinaire.

Articulant approches quanti- et qualitative, le dispositif de recherche s'est déployé sur deux années scolaires et demie (de septembre 2015 à décembre 2018). Lors de la première phase, une quinzaine d'écoles organisant un DASPA ont été prélevées. Un test évaluant les compétences en lecture et en écriture a été soumis à trois reprises aux 600 élèves de l'échantillon, afin de mesurer l'évolution des élèves sur une année scolaire (2015-2016). C'est à l'appui des résultats émanant des tests que, lors d'une seconde phase, deux écoles ont été sélectionnées afin de servir de terrain à l'analyse qualitative. Les données qualitatives concernant ces deux DASPA ont été collectées au moyen des méthodes traditionnelles de l'ethnographie : afin d'accéder aux pratiques effectives, la chercheuse a été amenée à observer et à filmer pendant deux semaines des leçons de français, de mathématiques, d'histoire-géographie et de religion. Elle a également recueilli les documents distribués aux élèves lors de ces séances, ainsi que des documents plus généraux (grilles-horaires, document d'intentions pédagogiques, planification de matière). Les entretiens qui ont suivi avec les enseignants ont permis de recueillir leur point de vue sur les pratiques observées. D’autres interviews ont ensuite été réalisées avec les élèves de DASPA mais aussi avec les enseignants des classes ordinaires, la direction des deux écoles et les éducateurs. Les données recueillies sous la forme de vidéos et d'entretiens ont dans un premier temps été retranscrites, puis traitées à l'aide d'un synopsis (Schneuwly, Dolz et Ronveaux, 2006). Le repérage d'« épisodes critiques » de classe, c'est-à-dire des moments où surgissent des « obstacles » significatifs à l'enseignement et à l'apprentissage (Astolfi, 1992 ; Brousseau, 1983), a constitué le point de départ à l'analyse qualitative. Décrits puis analysés, ces obstacles ont, dans un dernier temps, été réinterprétés à la lumière des gestes professionnels (Bucheton et Soulé, 2009) que les enseignants, avec ou sans succès, mettent en œuvre pour les surmonter. 
L'analyse du matériau empirique a permis de mettre en évidence un écart de pratique important entre les deux écoles. Cette divergence de pratiques a pu être en partie expliquée par une série de facteurs, relevant notamment du champ des représentations sociales. Il est notamment apparu qu'un nombre important d'enseignants adhéraient à la représentation de l'étranger/allophone vulnérable. Ce rapprochement, en l'espèce, peut trouver à se justifier. Les enfants et les adolescents bénéficiant du dispositif sont de nationalité étrangère et répondent à un statut particulier (demandeurs d'asile, réfugiés, ressortissants d'un pays en voie de développement, ...). C'est d'ailleurs leur arrivée depuis moins d'un an en Belgique, parce qu'elle les trouve démunis face à la langue de l'enseignement, qui justifie leur présence dans le dispositif.

Cependant, par-delà les faits concrets qui la corroborent, cette représentation, lorsqu'elle est trop unanimement partagée par le corps enseignant, au point de la faire primer sur les objectifs d'enseignement et d'insertion scolaire, peut entrainer des répercussions dangereuses sur les plans pédagogique et didactique. Notamment parce que sont alors favorisées:

- l'adoption de postures professionnelles qui s'écartent de celle de professeur. Un certain nombre d'enseignants rencontrés se déclarent tantôt « psychologues », tantôt « assistants sociaux», «éducateurs », etc.

- la poursuite d'objectifs autres que scolaires. Certains de nos informateurs allouent au DASPA une visée purement « sociale », semblable à celles que poursuit l'enseignement de promotion sociale;

- une diminution des attentes scolaires à l'égard de ce public. Certains enseignants (autres que les professeurs de français) vont jusqu'à attribuer à leur cours une fonction strictement récréative.

Il n'est alors pas rare que cet amenuisement des exigences scolaires entraine l'adoption de pratiques enseignantes que nous avons appelées « simplificatrices » : des pratiques « réflexes » mais peu réflexives, et donc bien souvent stériles en ce qu'elles tendent à réduire la difficulté supposée des apprentissages sans que cette simplification n’aboutisse à un réel bénéfice didactique. Dans la gamme des pratiques simplificatrices les plus spontanément mises en œuvre, on retrouve: des interactions professeur-élèves restreintes et à la portée limitée, une utilisation d'expédients limitant l'usage du langage par l'élève (mimiques, réponses monosyllabiques ou asyntaxiques, « oui/non »), une éviction des opérations discursives complexes (telles que « définir », « justifier », « reformuler ») à la faveur d'opérations simples ou déictiques (nommer ou désigner, par exemple), une absence de progression marquée entre les activités proposées, une propension des enseignants à puiser dans les contenus les plus élémentaires de la discipline, une prédominance du format de l'exercice sur celui de la tâche, une propension à improviser la leçon d'un bout à l'autre, etc. En se systématisant, ces pratiques conduisent la plupart du temps à des situations tantôt adidactiques, tantôt très (trop) faiblement didactiques, qui rendent l'enseignement peu à même de faire progresser ces élèves. 
Ce sur-ajustement aux difficultés et caractéristiques des élèves est également l'un des résultats de l'enquête menée dans le cadre de la recherche doctorale de El Karouni $(2010,2012)$ au sein de deux classes de $5^{e}$ générale de l'enseignement secondaire. La chercheuse formulait ainsi l'hypothèse que les pratiques des enseignants sont influencées par leurs représentations des compétences et des pratiques langagières des élèves catégorisés comme issus de l'immigration, représentations qui sont notamment fondées sur leurs perceptions de leurs caractéristiques sociolinguistiques et ethniques. Elle a ainsi examiné comment les objectifs linguistiques sont définis et par quels moyens les enseignants vont tenter de les faire acquérir par leurs élèves. Cette dimension a été explorée à partir du dispositif méthodologique suivant (voir El Karouni, Collès et Lucchini, 2008 pour un descriptif détaillé). Dans la première phase de l'enquête de terrain, l'approche a été essentiellement quantitative et a concerné quatre classes de $5^{e}$ générale de l'enseignement secondaire (la moyenne d'âge des élèves est de 16 ans et demi), contrastées du point de vue socioethnique. Le champ d'observation a été réduit à deux classes fréquentées exclusivement par des élèves issus de l'immigration ${ }^{7}$ où les résultats obtenus par les élèves à une évaluation en langue ${ }^{8}$ sont les plus faibles et où les exigences linguistiques manifestées par les professeurs de français lors de cette passation sont contrastées ; l'une «surévalue » et l'autre «sous-évalue » les performances objectives de ses élèves. Ces postures de «tolérance » et de « sévérité » ont suscité des questionnements qui ont fait l'objet d'une analyse qualitative. L'exploration des questions suscitées par la première phase de l'enquête de terrain a été qualitative et examinée à travers des études de cas menées en parallèle dans les deux contextes. Plus précisément, les modalités d'ajustement aux caractéristiques ethniques et sociolinguistiques des élèves sont examinées à travers l'étude combinée des représentations et des pratiques enseignantes. Les données ont été recueillies dans les contextes naturels et émanent des questionnaires, des observations directes des pratiques d'enseignement et des entretiens semi-directifs conduits avec le professeur et les élèves.

El Karouni a ainsi montré comment la représentation des élèves comme «non francophones » avait des répercussions négatives dans les pratiques d'enseignement du français. L'information déduite de l'origine migratoire et nationale de l'élève est corrélée à une pratique linguistique donnée. Dit autrement, à l'origine nationale des élèves est associée une série de caractéristiques concernant les pratiques linguistiques.

L'hétérocatégorisation des élèves enclenche un mécanisme que l'on peut décrire comme suit. À partir de cette information (erronée en grande part), l'enseignante développe l'attente que ses élèves ne progresseront pas dans leur connaissance du code linguistique : cette prophétie se manifeste de manière directe à travers la quantité de travail exigée et produite (les pratiques d'évaluation sont

7. Ces deux classes appartiennent à des établissements inscrits en « discrimination positive », lesquels se caractérisent par l'appartenance des élèves à des quartiers défavorisés qui présentent un indice socioéconomique faible.

8. Une épreuve sur des savoirs linguistiques décontextualisés et la rédaction d'une lettre de demande argumentée adressée au bourgmestre ont été utilisées comme outils d'évaluation. Les lettres de demande argumentées ont été évaluées par trois binômes de correcteurs extérieurs sur la base d'une même grille d'évaluation et par les enseignantes de français de chacune des quatre classes selon leurs propres critères. 
axées sur les paliers primaires dans l'échelle des difficultés ; les démarches intellectuelles d'apprentissage sollicitées sont élémentaires ; les savoirs disciplinaires sont peu investis) mais aussi indirecte (l'analyse des interactions révèle des logiques d'accommodation contre-productives dans la construction de la norme) qui donnent peu d'occasions, pour les élèves, d'apprendre et d'améliorer leur performance en langue.

Un autre travers de cette fusion des données ethniques et sociolinguistiques dans les représentations réside dans la réification d'une catégorie ethnolinguistique qui n'est pas pertinente dans la triangulation didactique des apprentissages, et qui est justement celle de francophones vulnérables, apparaissant dans la brochure de la Fédération Wallonie-Bruxelles publiée en 2014 et déjà mentionnée. Comme nous l'avons développé par ailleurs (El Karouni, 2017), ces derniers, peut-on y lire, «se perçoivent souvent comme Belges francophones. Certains d'entre eux ont été primo arrivants et scolarisés en classe passerelle ou en DASPA, d'autres appartiennent aux $2^{\mathrm{e}}$ ou $3^{\mathrm{e}}$, voire $4^{\mathrm{e}}$ ou $5^{\mathrm{e}}$ génération de l'immigration, d'autres encore sont d'origine belge » (p. 7). Les élèves sont caractérisés dans un registre plus subjectif qu'objectif: on ne dit pas de ces élèves qu'ils sont «Belges francophones » mais qu'ils «se perçoivent » comme tels. Le curseur n'est pas placé sur le statut administratif - être de nationalité belge est un fait objectivable - mais sur l'adéquation - moins objectivable car placée sur le plan de la perception - aux caractéristiques du « Belge francophone » qui fonctionne à cet endroit comme une catégorie ethnolinguistique.

Ces observations plaident, nous semble-t-il, pour que dans les discours la nature des besoins linguistiques en termes d'apprentissage soient dissociées de l'origine nationale des élèves. Ce changement de discours peut être soutenu par la pratique systématique d'une évaluation de type diagnostic à propos des ressources linguistiques disponibles et des besoins en langue.

\section{L'affaiblissement du sentiment d'appartenance à la langue com- mune et plus largement au pays de résidence}

Les catégorisations n'ont pas seulement un effet sur le parcours scolaire et l'acquisition linguistique. Elles ont aussi un effet sur la cohésion sociale des composantes ethniques de la société. Le sentiment d'appartenance à la langue commune et plus largement au pays de résidence (Lucchini et al., 2008) ressort affaibli par l'attribution abusive d'une incompétence linguistique sur la base de marqueurs ethniques tels que la couleur ou le nom, même chez les personnes nées et scolarisées en Belgique francophone.

Dans la recherche menée à la fin des années 2000 et mentionnée supra, nous interrogions le rapport au français de la population issue de l'immigration de la première, deuxième et troisième génération. Ont été interrogées 65 personnes originaires de 19 pays (39 à Bruxelles et 24 dans la partie francophone du pays) ; 22 de première, 43 de deuxième et 5 de troisième génération ; 33 hommes et 32 femmes ; 41 personnes âgées entre 30 et 49 ans, 19 de moins de 30 ans et 5 de plus de $50 ; 46$ 
diplômés de l'enseignement secondaire au moins. La recherche visait à décrire les pratiques linguistiques et à comprendre le système des représentations qui portent sur ces pratiques (statuts, valeurs, caractéristiques attribués aux langues, sentiment d'appartenance aux communautés, affects reliés à la pratique des langues, etc.). Les entretiens semi-dirigés ont été menés à partir d'un guide qui comprenait sept volets visant à décrire a) les parcours migratoires, b) les pratiques linguistiques, les statuts des langues parlées, les valeurs qui y sont rattachées et les motivations sous-jacentes, c) les usages et les souhaits concernant les « langues d'origine » (nous avons choisi d'utiliser cette dénomination dans les entretiens), d) la perception du plurilinguisme, e) le sentiment d'appropriation de la langue française, f) la question de la norme linguistique, g) les rapports entre les langues et la réussite scolaire et professionnelle.

L'association hétéroattribuée entre minorité visible, étranger et mauvaise connaissance du français a été soulignée par plusieurs informateurs comme étant à l'origine de pratiques de discrimination indirectes, générées par ces représentations négatives d'une non-correspondance au prototype «blanc-bleu-blond ». Ces pratiques de discrimination ont par ailleurs été corroborées par une série d'études, en Belgique ou non (v. le rapport de l'OCDE de 2008, qui conclut à une inégalité importante entre la population immigrée ou d'origine immigrée en ce qui concerne l'accès à l'emploi, en particulier pour les femmes, et également pour les générations scolarisées en Belgique ; v. également la recherche déjà citée menée en Suisse romande [Prikhodkine et al., 2008]).

Ce que la recherche mentionnée a laissé émerger, c'est que les personnes qui subissent ces pratiques de discrimination vont chercher à se former une image d'eux-mêmes cohérente et positive en récupérant d'autres marques ethniques. Parmi celles-ci, les langues associées à l'origine. Elles vont être réactivées ou apprises, qu'elles aient été ou non parlées par des ancêtres parce qu'elles vont permettre la reconnaissance réciproque à un groupe humain, accueillant cette fois, et vaste. Pour cette raison les langues standard, pouvant être transnationales, vont être privilégiées. Ce sont ces langues qui vont faire l'objet d'un investissement affectif.

A contrario, il apparait clairement que ce processus a comme conséquence un affaiblissement du sentiment d'appartenance à la société de résidence et à sa langue. Cela produit un désinvestissement affectif par rapport à la langue française : cette population, immigrée ou issue de l'immigration, se voit en quelque sorte expropriée du français. Au point que des personnes peuvent hésiter à se définir francophones, même quand le français est la seule langue qu'ils parlent. La valence affective attribuée aux langues d'une origine proche ou lointaine est niée au français, qui devient une langue nécessaire et utilitaire, comme langue de l'espace public, et qui en quelque sorte s'affaiblit. Dans tous ces cas, le regard qui caractérise l'autre comme un « allophone » et « vulnérable » finit par produire la vulnérabilité attribuée, en termes d'acquisition de la langue et de désinvestissement affectif. 


\section{Dissocier allophonie et vulnérabilité}

L'association entre allophonie et vulnérabilité replace le focus sur les manquements, les déficits ou les « handicaps » des élèves primoarrivants au lieu de se concentrer sur le renforcement des compétences ou des aptitudes déjà existantes.

En cela, il est à regretter que les outils de travail très concrets fournis par le Conseil de l'Europe, à travers sa politique linguistique et la publication du CECRL, soient très peu exploités par les écoles. Nous retiendrons ici deux concepts particulièrement intéressants : la définition positive de la compétence (CECRL, 2001) ainsi que le plurilinguisme et l'éducation plurilingue?.

En effet, dans le CECRL, la définition des compétences est toujours positive. Les niveaux sont définis par rapport à ce que l'apprenant est capable de faire et non pas à ce qui est attendu (norme) ou à ce qu'il n'est pas encore en mesure de faire (maitrise/non maitrise). Il accepte que les compétences puissent être partielles et il laisse la place au développement d'interlangues, ce qui nous permet de travailler différemment en classe et de mieux évaluer les acquis. Une application mécanique et artificielle des niveaux ou des compétences attendues pour, par exemple, avoir la nationalité (A2) ou pour intégrer une classe « ordinaire » (B1) trahit cet esprit et ne donne pas les résultats souvent souhaités par les enseignants, c'est-à-dire des classes homogènes sur le plan de la « maitrise de la langue ».

Même si, sur le plan didactique, le CECRL (2001) peut s'avérer très intéressant, le concept du plurilinguisme et de l'éducation plurilingue offre un changement de vision et de paradigme, d'une école unilingue et uniculturelle qui peine à enseigner même une deuxième langue, à une école où toutes les langues sont possibles. La compétence plurilingue comprend toutes les langues et les variétés linguistiques du répertoire d'un individu. Ces compétences sont de nature et de niveau différents seIon les langues au sein de son répertoire allant du balbutiement de quelques mots dans une langue à un contrôle quasi total d'une autre. La compétence plurilingue dépend des contextes d'utilisation et des besoins langagiers des usagers. Dans ce sens, un individu pourrait faire des études en ingénierie dans une langue sans nécessairement pouvoir tenir une conversation « sociale» dans cette langue.

L'éducation plurilingue, selon Beacco et Byram (2007), encourage la prise de conscience du pourquoi et du comment on apprend les langues cibles; la prise de conscience de compétences transposables et de la capacité à les réutiliser dans l'apprentissage des langues ; le respect du plurilinguisme d'autrui et de la reconnaissance des langues et de leurs variétés, quelle que soit l'image qu'elles ont dans la société ; le respect des cultures inhérentes aux langues et de l'identité culturelle d'autrui ; la capacité à percevoir et à assurer le lien entre les langues et les cultures; une approche intégrée de l'enseignement des langues dans les curricula.

9. Information disponible sur www.coe.int/lang/fr. [Page consultée le 30 mars 2021.] 
Il nous parait évident que ce changement de paradigme dans les écoles ne sera pas possible sans une rupture dans la formation, initiale et continue, des enseignants. Afin d'y arriver, une approche intégrée est nécessaire non seulement pour l'enseignement des langues mais aussi dans les curricula des formations universitaires et supérieures.

Le plurilinguisme et l'éducation plurilingue sont des concepts qui peuvent certainement soutenir un regard positif sur la diversité linguistique et culturelle. Mais pour dissocier davantage le public primoarrivant et d'autres publics en difficulté scolaire, et l'allophonie de la vulnérabilité, une recherche en cours déjà mentionnée (Maxime Alais) développe de nouvelles mises en liens entre le public primoarrivant scolarisé en FWB et d'autres publics qui doivent apprendre une nouvelle langue de l'école, notamment celui du réseau AEFE (Agence pour l'enseignement français à l'étranger), en mettant l'accent sur le concept d'altérité linguistique et culturelle, et non plus sur la notion d'allophonie seule, puisqu'elle est employée pour séparer, dans les discours et dans les pratiques, certains élèves du public scolaire considéré dans son ensemble. Pour Auger (2019) également, d'ailleurs, l'emploi de la notion d'allophonie témoigne d'un refus de reconnaissance du plurilinguisme et de l'expertise des élèves primoarrivants. La recherche décrite ci-après tente de penser autrement l'altérité linguistique et culturelle des élèves que sous le prisme seul de la notion d'allophonie.

Le regard usuel porté sur la scolarisation des apprenants primoarrivants en FWB est largement problématique : il insiste sur des caractères déficitaires et (très) spécifiques, a minima non ordinaires de ce public. Or, ce regard déficitaire assigne et homogénéise ce public scolaire, pourtant fondamentalement pluriel. Pour renouveler ce regard qui assigne l'autre, le choix de cette recherche en cours (et son originalité) a été de parier sur la comparaison entre la situation de DASPA en FWB et celles d'écoles appartenant aux réseaux AEFE ou CIS (Council of International Schools). Ces deux dernières situations sont habituellement considérées disjointes ou très différentes de celle qui concerne la scolarisation d'apprenants primoarrivants. Pourtant, elles ont un point commun majeur avec celle-ci : la langue de l'école y est, pour une majorité de leurs apprenants, une langue étrangère/seconde. Or, cette scolarisation dans une autre langue semble, dans ces deux situations de comparaison, présentée et/ou vécue de manière beaucoup plus positive par les acteurs éducatifs et par les apprenants.

Nous avons donc dans un premier temps comparé des discours officiels issus des trois situations retenues, puis effectué des entretiens et des observations dans quatre écoles : deux écoles belges francophones bénéficiant d'un DASPA, une école du réseau AEFE située en Asie de l'Est et une école du réseau CIS située en Belgique. Concernant l'interprétation des discours, des extraits de ceux-ci ont été sélectionnés après un relevé d'occurrences de termes-clés, puis comparés. Des entretiens compréhensifs (Kaufmann, 2014) ont ensuite été menés dans les quatre écoles retenues avec les directions des écoles, des enseignants et des apprenants, ainsi que des observations en situation (Martineau, 2005). Pendant les entretiens, des extraits de discours sélectionnés après relevés d'occurrence ont notamment été utilisés comme déclencheurs de discussion. Pour l'interprétation des discours, la passation des entretiens et les observations effectuées, nous avons mis à profit notre expérience professionnelle personnelle d'enseignant de français dans un des deux DASPA retenu, 
en « Assum[ant] la recherche comme teintée dans la masse par la présence et l'expérience des chercheurs » (Huver, 2014:23; voir aussi Castellotti, 2017 : 10). Par ailleurs, notre démarche comparatiste s'inscrit dans la perspective herméneutique telle que défendue par Detienne (2000) et par Jucquois (2000). Pour celui-ci,

Le comparatisme instaure l'obligation d'une multiplicité de regards sur un objet supposé identique. Il débouche sur une prise de conscience de la complexité, propriété qui, lorsqu'elle concerne l'homme, entraîne une perception plus aigüe de la diversité et du nécessaire pluralisme qui en découle. (Jucquois, $2000: 18-19)$

La comparaison/le croisement entre des terrains et des publics scolaires usuellement considérés disjoints (les primoarrivants «migrants » associés aux « vulnérables » d'un côté, les riches « expatriés » et «natifs » privilégiés de l'autre) renouvelle les discours déficitaires habituellement tenus à propos des apprenants primoarrivants et éclaire une problématique de reconnaissance de l'altérité linguistique et culturelle. Par ailleurs, confrontés à des discours institutionnels bien davantage positivants face à la diversité/l'altérité linguistique et culturelle, les acteurs de terrain développent un regard beaucoup moins négatif/déficitaire sur la scolarisation d'apprenants dits allophones.

Mais les observations effectuées sur un terrain scolaire AEFE montrent qu'au-delà des discours communicants/promotionnels tenus par les institutions, les apprenants qui doivent apprendre une nouvelle langue de l'école échappent rarement à une mise en altérité, quel que soit le contexte scolaire. Ces différentes situations/institutions scolaires gagneraient donc à entrer en relation, à échanger, à développer des partenariats, etc., pour vivifier les discours qu'elles tiennent à propos des élèves qui doivent apprendre une nouvelle langue de l'école, pour diversifier leurs pratiques didactiques; pour interroger cette mise en altérité souvent observée.

Enfin, cette recherche met en évidence un partage de la problématique de la reconnaissance de l'altérité qui dépasse largement le seul critère de la langue « autre » comme la notion d'allophonie peut le laisser entendre. La notion d'allophonie n'est-elle pas une réduction de la notion d'altérité ? L'altérité linguistique et culturelle n'est-elle pas unanimement partagée entre nous tous, même lorsque nous parlons la même langue ? Plutôt que de la réserver aux " autres », ne faudrait-il pas mettre en avant son partage entre tous? En ce sens, l'école n'est-elle pas elle aussi allophone face aux apprenants visés par les dispositifs mentionnés? Autres problématiques que nous approfondirons dans un autre article. 


\section{Références}

Acquisition des langues et immigration (ALIA) (2018), "Politiques linguistiques dans les contextes multilingues de la Belgique francophone : aide ou ségrégation ? », Synergies Pays germanophones, n 11, p. 185-197.

Astolfi, Jean-Pierre (1992), «Apprendre parfranchissement d’obstacles ? », dans Hélène Romian (dir.), Repères, recherches en didactique du français langue maternelle, n5, p. 103-116.

Auger, Nathalie (2010), Élèves nouvellement arrivés en France, Paris, Éditions des archives contemporaines.

Auger, Nathalie (2019), "Allophone” : trajectoire d'une catégorie aux prises avec la notion d'expertise », TRANEL : Travaux neuchâtelois de linguistique, vol. 70 (L'expertise dans le champ des sciences du langage sur des terrains sensibles: un point de vue épistémologique, sous la dir. de Nathalie Auger et Maud Verdier), p. 23-41.

Beauchemin, Cris, Christelle Hamel, Maud Lesné, Patrick Simon et al. (2010), « Les discriminations : une question de minorités visibles », Populations et Sociétés, n 466.

Beacco, Jean-Claude et Michael Byram (2007), De la diversité linguistique à l'éducation plurilingue: guide pour l'élaboration des politiques linguistiques éducatives en Europe, disponible sur www.coe.int/fr/web/platform-plurilingual-intercultural-language-education/three-fundamental-and-complementary-tools-for-the-implementation-of-plurilingual-education. [Page consultée le 20 août 2020.]

Brousseau, Guy (1983), "Les obstacles épistémologiques et les problèmes en mathématiques », Recherche en didactique des mathématiques, vol. 4, n² 2, p. 164-198.

Bucheton, Dominique et Yves Soulé (2009), «Les gestes professionnels et le jeu des postures de l'enseignant dans la classe : un multi-agenda de préoccupations enchâssées », Éducation et didactique, vol. 3, n³, p. 29-48.

Castellotti, Véronique (2017), Pour une didactique de l'appropriation : diversité, compréhension, relation, Paris, Didier.

Conseil de l'Europe (2001), Cadre européen commun de référence pour les langues : apprendre, enseigner, évaluer, disponible sur www.coe.int/fr/web/common-european-framework-reference-languages. [Page consultée le 20 août 2020.]

Detienne, Marcel (2000), Comparerl'incomparable: oser expérimenter et construire, Paris, Seuil.

El Karouni, Salima (2012), «L'enseignement du français en contextes scolaires hétérogènes : pour un renouvellement des repères didactiques traditionnels », Recherches et Éducations, nº 7, p. 149165.

El Karouni, Salima (2017), «La place de l'ethnicité au sein du champ de l'enseignement/apprentissage du français », Études en didactique des langues, n²8, p. 59-80. 
Fédération Wallonie-Bruxelles (2014), Enseigner aux élèves qui ne maitrisent pas la langue d'enseignement, disponible sur http://www.enseignement.be/index.php?page=0\&navi=3627. [Page consultée le 20 août 2020.]

Fédération Wallonie-Bruxelles (2019), Décret visant à l'accueil, la scolarisation et l'accompagnement des élèves qui ne maitrisent pas la langue dans l'enseignement organisé ou subventionné par la Communauté française, disponible sur https://www.gallilex.cfwb.be/document/ pdf/46275_000.pdf. [Page consultée le 20 août 2020.]

Galligani, Stéphanie (2012), « Regards croisés sur les enfants venus d'ailleurs et scolarisés en France », Les Cahiers du GEPE, $n^{\circ} 4$ (Les langues des enfants «issus de l'immigration» dans le champ éducatif français), disponible sur http://cahiersdugepe.fr/index.php?id=2314\#texte. [Page consultée le 25 octobre 2020.]

Honneth, Axel (1992), La lutte pour la reconnaissance, Paris, Gallimard.

Huver, Emmanuelle (2015), « Prendre la diversité au sérieux en didactique/didactologie des langues. Altériser, instabiliser: quels enjeux pour la recherche et l'intervention? 》, dans Emmanuelle Huver et David Bel (dir.), Prendre la diversité au sérieux en didactique/didactologie des langues. Altériser, instabiliser: quels enjeux pour la recherche et l'intervention? Paris, L'Harmattan, p. 11-24.

Jucquois, Guy (2000), "Le comparatisme, éléments pour une théorie », dans Guy Jucquois et Christophe Vielle (dir.), Le comparatisme dans les sciences de l'homme : approches pluridisciplinaires, Bruxelles, De Boeck, p. 17-46.

Kaufmann, Jean-Claude (2014), L'entretien compréhensif, Paris, Armand Colin.

Klein, Wolfgang (1989), L'acquisition de langue étrangère, Paris, Armand Colin.

Lucchini, Silvia (2005), «L'enfant entre plusieurs langues : à la recherche d'une langue de référence », Enfance, vol. 57, n 4, p. 299-317.

Lucchini, Silvia (2012), « De la langue à la cohésion sociale ou de la cohésion sociale aux langues?», dans Virginie Conti, Jean-François De Pietro et Marinette Matthey (dir.), Langue et cohésion sociale : enjeux politiques et réponses de terrain, Neuchâtel, Délégation à la langue française, p. 87110.

Lucchini, Silvia, Philippe Hambye, Gilles Forlot et Isabelle Delcourt (2008), «Francophones et plurilingues: le rapport au français et au plurilinguisme des Belges issus de l'immigration », Français et société, no 19, p. 1-90.

Maravelaki, Aphrodite (2007), «Les classes-passerelles en Belgique francophone : vers une transformation de l'école uni-culturelle? », dans Silvia Lucchini et Aphrodite Maravelaki (dir.), Langue scolaire, diversité linguistique et interculturalité, Cortil-Wodon, Éditions modulaires européennes, p. 195-212.

Martineau, Stéphane (2005), «L'observation en situation : enjeux, possibilités et limites », Recherches qualitatives, hors-série $n^{\circ} 2$ (Actes du colloque "L'instrumentation dans la collecte des données 》 tenu à l'Université du Québec à Trois-Rivières le 26 novembre 2004), p.5-17. 
Merle, Pierre (2012), La ségrégation scolaire, Paris, La Découverte.

OCDE (2008), Les migrants et l'emploi, vol. 2 : l'intégration sur le marché du travail en Belgique, en France, aux Pays-Bas et au Portugal, disponible sur www.oecd.org/fr/belgique/lesmigrantsetlemploivol2lintegrationsurlemarchedutravailenbelgiqueenfranceauxpays-basetauportugal. htm. [Page consultée le 25 août 2020.]

Oger, Élodie (2019), Faire-passerelle : analyse systémique des pratiques enseignantes dans les dispositifs d'accueil et de scolarisation des élèves primoarrivants de Belgique francophone, thèse de doctorat, Louvain-la-Neuve, Université catholique de Louvain.

Prikhodkine, Alexei, Pascal Singy, Noémie Charton et Alessandro lannelli (dir.) (2008), « Tout nom n'est pas bon à dire : discriminations à l'embauche en Suisse romande », numéro thématique du Bulletin de linguistique et des sciences du langage, nº 24.

Santocono, Girolamo (1987), Rue des Italiens, Mons, Éditions du Cérisier.

Schneuwly, Bernard, Joaquim Dolz et Christophe Ronveaux (2006), « Le synopsis : un outil pour analyser les objets enseignés », dans Marie-Jeanne Perrin-Glorian et Yves Reuter (dir.), Les méthodes de recherche en didactiques : actes du premier séminaire international sur les méthodes de recherches en didactiques de juin 2005, Villeneuve d'Asca, Presses universitaires du Septentrion, p. 175-189. 


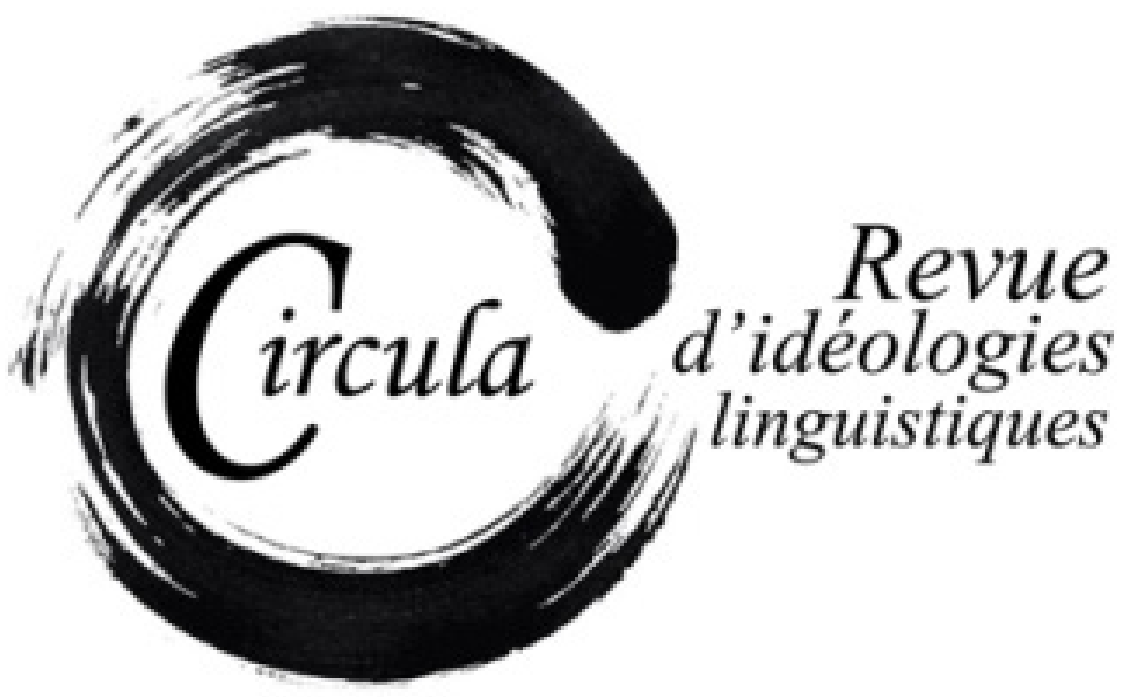

TITRE: LES EXPÉRIENCES ARTISTIQUES EN CONTEXTE PLURILINGUE : UNE MISE À L'ÉPREUVE DE LA VULNÉRABILITÉ LINGUISTIQUE?

Auteur: Maud SÉrusclat-Natale, Université Paul Valéry Montpellier III

Revue: CIRCula, NumÉro 12 : LA VULNÉRABILITÉ LINGUISTIQUE

Directrice: Claudia Torres CAStillo

PAGES: $107-131$

ISSN: 2369-6761

URI: HTTP://HDL.HANDLE.NET/11143/18445

DOI: HTTPS://DOI.ORG/10.17118/11143/18445 


\title{
Les expériences artistiques en contexte plurilingue : une mise à l'épreuve de la vulnérabilité linguistique?
}

Maud Sérusclat-Natale, Université Paul Valéry Montpellier III m.natale@mascene.eu

Résumé : «Parlemonde », festival artistique, a réuni 15 artistes et 250 participants plurilingues, majoritairement récemment arrivés en France. Né d'une collaboration entre une scène nationale et l'école, «Parlemonde » fait de la diversité linguistique et culturelle d'un territoire un catalyseur de création. Si les pratiques artistiques sont encouragées pour l'appropriation d'une nouvelle langue, elles demeurent à l'école considérées comme une épreuve risquée, une mise en danger ou une (sur) exposition de ces élèves a priori vulnérables du fait de leur qualité d'apprenants de français et de leur expérience de l'exil. Nous montrerons qu'elles révèlent les processus de construction des situations de vulnérabilité (linguistique, culturelle, scolaire) et qu'elles contribuent, à certaines conditions, à les désamorcer en légitimant les répertoires langagiers et en créant, par l'exposition publique, les conditions de dépassement du sentiment d'insécurité linguistique et culturelle, transcendant la vulnérabilité en une reconnaissance commune des forces de chacun (adolescents, enseignants, artistes et publics).

Mots-clés : Élève allophone arrivant ; représentations ; expériences artistiques ; vulnérabilité ; appropriation

\begin{abstract}
Parlemonde", an artistic festival, brought together 15 artists and 250 multilingual participants, most of whom had recently arrived in France. Born from a collaboration between a national theater and public school, "Parlemonde" makes the linguistic and cultural diversity of a territory a catalyst for creation. Although artistic practices for the appropriation of a new language are encouraged, in school they are still considered a risky ordeal and an endangerment or (over)exposure of these seemingly vulnerable students, due to their status as learners and their experience of exile. We will demonstrate, on the one hand, that they expose the processes of construction of situations of vulnerability (linguistic, cultural, academic) and that they contribute, under certain conditions, to their deconstruction by legitimizing the languages and by creating, through public exposure, the conditions to overcome the feeling of linguistic and cultural insecurity; transcending vulnerability into a common recognition of each person's strengths (teenagers, teachers, artists and audiences).
\end{abstract}

Key words: newly arrived pupil; representation; artistic experiences; vulnerability; appropriation 


\section{Introduction}

Cette contribution repose sur notre recherche doctorale en cours, portant sur le festival Parlemonde se déroulant en biennale depuis 2017 à Montbéliard (France). Organisé par une scène nationale publique nommée «MA scène nationale ${ }^{1}$ » (désormais MA) en collaboration avec le Rectorat de l'académie de Besançon, particulièrement avec les services dédiés à l'action culturelle DAAC ${ }^{2}$ et le CASNAV ${ }^{3}$, Parlemonde est un programme de résidences d'artistes qui ont lieu majoritairement en milieu scolaire, et qui entend favoriser l'appropriation de la langue française et l'inclusion des élèves allophones nouvellement arrivés (désormais EANA). Il se fonde sur l'idée que les plurilinguismes de tous (artistes, participants, enseignants, etc.) peuvent être un moteur de création artistique, de rencontre et de reconnaissance sociale.

Nous présenterons d'abord en quoi les EANA rencontrés lors de notre recherche et principaux participants de Parlemonde, sont parfois considérés comme « vulnérables » ou en situation de vulnérabilité linguistique, scolaire et plus largement sociale, par les adultes collaborant avec eux. En effet, «barrière de la langue », fragilité psychologique ou traumatismes liés à l'exil (Mestre 2015 ; OCDE 2008) semblent enfermer les EANA dans un continuum de l'empêchement que l'urgence de la diplomation et la nécessité de rentabilité scolaire visant l'insertion professionnelle viennent renforcer. Nous analyserons ces obstacles systémiques souvent avancés pour justifier le fait que leur participation à un projet artistique public constitue un risque qui les surexpose, et qui pourrait accroitre leur vulnérabilité. Nous montrerons au contraire que les expériences artistiques conçues en partenariat sont vectrices d'empowerment (Sen, 2009) et transforment tant ceux qui les exercent que ceux qui les reçoivent. Notre approche, ethnographique, reposera principalement sur les outils de l'analyse du discours. Nous utiliserons un corpus d'entretiens semi-directifs, nos notes de terrain et des exemples de créations artistiques de Parlemonde pour expliquer comment ce type d'actions peut contribuer, à certaines conditions, à favoriser l'entrée dans la langue de scolarisation pour les EANA impliqués, mais également à valoriser les plurilinguismes devenant vecteurs de cohésion sociale, réveillant un patrimoine commun dans un territoire riche d'une histoire migratoire ancienne relativement ignorée.

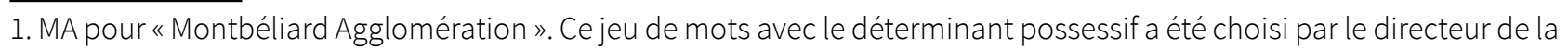
structure pour renforcer l'idée centrale de son projet qui vise à ce que les habitants s'approprient ce lieu de culture dédié à la création artistique contemporaine. Sa labellisation par l'État remonte à plus de 20 ans.

2. DAAC pour Délégation académique à l'action culturelle.

3. CASNAV pour Centre académique pour la scolarisation des enfants nouvellement arrivés et/ou issus de familles itinérantes ou de voyageurs. 


\section{2. (Dé)composer les situations de vulnérabilité}

Étymologiquement, la vulnérabilité est inhérente à la condition humaine, et signifie être exposé au risque de blessure. La catégorie de la vulnérabilité est de plus en plus utilisée en sciences sociales et humaines, ainsi qu'en droit. Le substantif « vulnérabilité » renvoie alors à un groupe humain hétéro-déterminé, repéré comme potentiellement plus fragile, en fonction d'un contexte ou d'un environnement donné. Le Conseil de l'Europe (COE), dans le but de construire des politiques communes de prévention, a désigné 3 groupes sociaux comme spécifiquement exposés à la vulnérabilité : les personnes en situation de handicap, les migrants et les enfants. Dans les travaux des Nations Unies, ces 3 groupes sont rejoints par les femmes et les populations autochtones ${ }^{4}$ tandis que dans les représentations sociales communément partagées, en particulier en temps de crise, sont vulnérables les jeunes (inexpérimentés et/ou imprudents), les plus anciens (fragilisés par l'âge) et les personnes économiquement précaires. Dans cette approche, l'adjectif « linguistique » adossé à vulnérabilité, renverrait au fait qu'un sujet ne soit pas en pleine capacité de communiquer et de s'exprimer avec le groupe dans lequel il est intégré. Sa vulnérabilité ne serait pas l'une de ses qualités propres, mais bien relative à un état lié à une situation vécue, ce qu'Ennuyer précise ainsi : «La vulnérabilitë d'un individu [est] occasionnée par l'interaction entre une personne et son environnement au sens large » (Ennuyer, 2017 : 371). On pourrait aller plus loin en ajoutant que la vulnérabilité linguistique d'un individu pourrait être occasionnée également par l'impossibilité (vécue, ressentie ou imaginée) de l'interaction avec cet environnement. Dans une société ou la « maitrise de la langue » est à la fois un moyen d'intégration et une condition de sélection pour l'octroi d'un titre de séjour par exemple, les populations allophones et déplacées sont désignées par les institutions comme vulnérables et le facteur linguistique est central : à la fois symptôme et cause de leur vulnérabilité sociale. En France comme ailleurs, nous nous situons dans un contexte où les institutions s'emparent de ce concept de vulnérabilité au titre de l'inclusion. Le projet de création artistique que nous analysons en fait dialoguer 2 : l'école et une structure culturelle publique. Elles partagent une mission de service public: l'éducation artistique et culturelle. Elles portent une attention particulière aux nouveaux arrivants, qu'elles désignent cependant de manière différente (nous y reviendrons) et elles se mobilisent pour être accessibles. Ce paradigme de l'accessibilité, ou de l'accessibilisation (Ebersold et ArmagnagueRoucher, 2017), est le pendant d'une politique d'inclusion et revêt différents aspects au cœur desquels la question des représentations est cruciale.

4. Information disponible sur http://www.claiminghumanrights.org/special_groups.html?L=1. [Page consultée le 2 octobre 2020.] 


\subsection{Les EANA : des apprenants vulnérables?}

En France, la scolarisation est un droit pour tous les enfants de 3 à 18 ans et l'instruction est obligatoire jusqu'à la majorité ${ }^{5}$ quelle que soit la situation légale, sociale ou linguistique de l'élève (et/ou de sa famille) sur le territoire. Depuis le Bulletin officiel (B.O.) de 2012, les modalités de scolarisation des EANA se font dans une visée inclusive, au moyen de dispositifs d'enseignement spécifiques appelés UPE2A (Unités pédagogiques pour les élèves allophones arrivants) ouverts sur la classe dite " ordinaire » et pilotés dans chaque région académique par les CASNAV. Les textes prévoient que les élèves soient inscrits selon leur âge dans la classe qui correspond. Ils y suivent certains cours selon leurs compétences et la quittent ponctuellement pour rejoindre l'UPE2A afin de bénéficier de cours spécifiques de français langue de scolarisation, et/ou de remise à niveau en diverses matières comme les mathématiques, l'anglais, les sciences, etc. Chaque élève accueilli a été préalablement évalué avec l'aide des CASNAV et bénéficie d'un emploi du temps individualisé pour répondre à ses «besoins éducatifs particuliers».

Dans les différents textes régissant cette organisation systémique, le terme de "vulnérabilité » n'apparait pas, au profit d'une politique de prise en compte des « besoins éducatifs particuliers » et de compensation faisant par exemple échos aux travaux du comité interministériel du handicap et de l'OCDE. Cette dernière catégorise 3 types de situations de besoins éducatifs particuliers : la déficience pour « les élèves présentant des déficiences ou incapacités » liées à des troubles médicaux; la difficulté pour les élèves présentant des « troubles du comportement ou des troubles affectifs et des apprentissages » desquels résultent des « problèmes d'interaction avec l'environnement éducatif » et les « désavantages », pour les élèves défavorisés en raison de «facteurs socio-économiques, culturels et/ou linguistiques, pour lesquels le besoin éducatif consiste à compenser les désavantages liés à ces facteurs » (OCDE, 2008 : 22). Cette dénomination a connu des évolutions mais demeure vivement critiquée par les chercheurs car elle associe de facto la question de l'allophonie à la non-maitrise de la langue de scolarisation et donc à un handicap conduisant à un « risque scolaire ». Cette conception viendrait renforcer la représentation sociale de l'EANA comme apprenant « vulnérable » car l'altérité demeure pensée par les institutions comme une situation de déficit (Métraux, 2011). Cela va à l'encontre de près de 30 ans de recherche sur le bi-plurilinguisme qui ont démontré que parler plusieurs langues, à des degrés divers, n'était pas une situation conduisant systématiquement à un handicap social mais plutôt un avantage cognitif, social et culturel considérable, s'il est correctement accompagné (Billiez, 1985 ; Lüdi et Py 1986 ; Cummins, 2001 ; Auger, 2010). Si le paradigme du « besoin éducatif particulier » reflète une volonté politique d'inclusion des EANA, il suppose une nécessaire adaptation du système scolaire devant s'appuyer sur - et produire - de nouvelles ressources pour scolariser ces publics «spécifiques ». Or, l'élève allophone arrivant en contexte scolaire est très souvent davantage pensé comme en situation de risque d'échec, et ce constat supplante la valorisation de son plurilinguisme et/ou de son bi-plurilinguisme émergeant (García, 2009) en tant que force

5. Information disponible sur https://www.service-public.fr/particuliers/vosdroits/F1898. [Page consultée le 2 octobre 2020.] 
potentielle d'apprentissage de la langue cible. De plus, la prise en compte des besoins particuliers et la mise en place de compensations adaptées ne vont pas de soi. Les difficultés présentées par les enseignants sont très souvent liées à l'évaluation ou à la certification, au motif d'une représentation de l'idéal républicain qui se réclamerait d'une équité de traitement confondue avec un principe d'égalité absolu rendant toute compensation comme une rupture de celle-ci. C'est la trace, consciente ou non, du modèle du creuset républicain et témoigne de la complexité de sa nécessaire transformation (Renaut, 2009). Cela construit des situations de vulnérabilité, pour les élèves, EANA inclus. Pour illustrer cela au niveau institutionnel, citons le rapport intitulé « Les aménagements d'épreuves d'examens pour les élèves et étudiants en situation de handicap » rédigé par l'Inspection générale en 2018. Sont mentionnées dès la page 3 les « difficultés éthiques pour faire respecter le droit, garantir l'équité de traitement et accompagner les jurys sans rompre avec les principes d'indépendance et d'égalité qui contribuent à la valeur du diplôme délivré » (Gavini-Chevet, Delécluse et Bigaut, 2018 : 3). Dans le cas des EANA, aucun aménagement d'épreuve n'est prévu, sauf, depuis 2017, pour l'une des épreuves écrites de la discipline «français » (la rédaction) du diplôme de fin de collège, pour laquelle les textes prévoient l'autorisation de l'usage d'un dictionnaire «éventuellement bilingue »'. Le rapport précise d'ailleurs qu'il y a des cas problématiques:

La même contribution fait état de prescriptions qui manquent de précision ou qui paraissent en dehors du champ règlementaire des attributions des médecins (l'exemple donné est la mise à disposition d'un dictionnaire pour un élève allophone qui n'était pas, par ailleurs, en situation de handicap avéré. (Gavini-Chevet, Delécluse et Bigaut, 2018: 14)

Cet exemple, parmi tant d'autres (Adam-Maillet et Sérusclat-Natale, 2019), témoigne du fait que l'école réactive l'idéologie d'un État monolingue, voire glottophobe et glottophage (Calvet, 1974 ; Blanchet, 2016; Escudé, 2018). Le système scolaire demeure pour les chercheurs « l'un des terrains privilégiés de l'analyse des discriminations linguistiques » selon les termes de Veret (2018: §3) dans son compte-rendu de Langues et discriminations. Il y souligne d'ailleurs que « la plupart des auteurs du numéro décrivent en effet l'école française comme le lieu d'une discrimination linguistique systématisée, qui perpétue "la matrice d'idéologie monolingue" (Escudé, 2018) autour de laquelle s'est forgé l'État-nation » (Veret, 2018 : § 3).

\subsection{Vulnérabilité, risque scolaire ou difficulté?}

Les travaux de recherche qui s'intéressent à la scolarité des EANA montrent que ces derniers sont très souvent associés au paradigme de la "difficulté scolaire », attribuée le plus souvent soit à un

6. Cette situation vient d'évoluer grâce à une note de la Direction Générale de l'enseignement scolaire, envoyée aux recteurs le 2 avril 2021. 
manque de scolarisation antérieure 7 , soit à un déficit de «maitrise de la langue », et parfois aux 2 facteurs. II a été démontré que ces discours reposaient souvent sur un manque de formation des enseignants (Auger, 2010, 2013 ; Goï, 2015), ce que relèvent également Armagnague et al. dans le rapport EVASCOL 2018 commandé par le Défenseur des droits. Ces représentations ont, pour Auger, « un impact majeur sur l'ensemble des partenaires du système scolaire : enseignants, enfants, parents, acteurs sociaux, etc. Elles sont souvent en décalage par rapport aux recherches en (socio)linguistique et en didactique des langues» (Auger, 2008 : 187). Elle ajoute que les discours « soulignent aussi davantage les difficultés que les éléments positifs de leur scolarisation» (Auger, 2010: 17).

Les principes de l'inclusion, inscrits en France dans la loi ${ }^{8}$ définissent un cadre juridique ambitieux, mais la « souplesse » - selon le terme du B.O. de 2012 - nécessaire à leur mise en place, manque sur le terrain. Cela place les EANA ne maitrisant pas encore la langue de scolarisation, en situation de vulnérabilité (linguistique, scolaire, et donc sociale). Ils sont de facto menacés d'exclusion, d'échec, alors même que leurs compétences linguistiques, scolaires, sociales, seraient invisibilisées par le système lui-même. Les sociologues Ebersold et Armagnague-Roucher décrivent ce phénomène ainsi :

La persistance des inégalités rappelle qu'un élève jugé à problème est celui qui pose problème aux acteurs du système éducatif du point de vue social. Les manières de faire et d'être de ceux dérogeant aux normes scolaires et aux exigences habituelles du métier d'élève bousculent les règles, les connaissances, les repères et les usages et situent les possibles scolaires dans le développement de stratégies éducatives complexes. Elles empêchent l'identification de routines permettant de conjuguer les exigences inhérentes à la personnalisation des pratiques aux enjeux identitaires et professionnels que demande la reconfiguration des rapports entre enseignant et élève. Elles remettent en cause l'effet miroir qui traverse toute relation sociale et interrogent les membres de la communauté scolaire dans leurs certitudes, questionnent l'image qu'ils se forgent de leur fonction, de leur rôle et d'eux-mêmes et engendrent des tâtonnements pédagogiques et des attitudes compassionnelles fondées sur les subjectivités individuelles. (Ebersold et Armagnague-Roucher, 2017 : 138)

Cet état des lieux est important à poser pour comprendre les enjeux de Parlemonde. Pour les 7 établissements scolaires impliqués, les objectifs qui figurent dans les « fiches actions ${ }^{9}$ » des projets Parlemonde (documents institutionnels à présenter lors des commissions validant non seulement la pertinence éducative du projet mais aussi les modalités de son financement) on trouve :

7. Voir Eduscol : "L'importance des dynamiques territoriales et d'un travail partenarial afin d'inscrire les élèves les plus vulnérables, notamment ceux qui ont été peu scolarisés antérieurement, dans une logique de parcours de formation, d'inclusion et d'insertion professionnelle », disponible sur https://eduscol.education.fr/cid73170/inclusion-scolaire-des-eleves-allophones-et-des-enfants-de-familles-itinerantes.html) [Page consultée le 2 octobre.]

8. Loi n² 2005-102 du 11 février 2005 pour l'égalité des droits et des chances

9. Ces textes sont co-écrits par les enseignants impliqués dans les projets et la structure culturelle puis visés par les chefs d'établissement. 
- Déségrégation des dispositifs UPE2A du lycée grâce et par le contact des élèves avec l'artiste dans une démarche de création au centre de laquelle on interrogera la question des langues.

- Favoriser l'inclusion et valoriser le plurilinguisme

- $\quad$ Circulation des élèves, dans les apprentissages (FLS et Pédagogie de projet).

- Travail artistique et interdisciplinaire sur la question de la trace, du territoire, et de l'orientation en valorisant le répertoire linguistique des participants et de l'établissement (élèves et équipes), notamment le patrimoine linguistique propre aux ateliers professionnels.

- Favoriser l'engagement dans les activités culturelles et dans le Français Langue de Scolarisation.

On note que les termes de « désegrégation » et de « circulation » font écho au concept d' « inclusion » et que le projet artistique apparait comme un potentiel facteur facilitant la reconnaissance d'un patrimoine linguistique existant, qu'il s'agit de positionner comme central et de patrimonialiser.

\subsection{Les EANA : un public cible mais « empêché »?}

Pour la structure, ces enjeux de patrimonialisation et de dialogue avec le territoire sont également importants. MA fait partie des institutions culturelles publiques décentralisées, plus précisément des 74 « scènes nationales ${ }^{10}$ » de France. Ces scènes possèdent 3 missions : soutenir la création contemporaine en finançant des résidences et en mettant à disposition leurs lieux et leurs équipes, diffuser des spectacles pluridisciplinaires issus des arts vivants et participer activement à l'éducation artistique du territoire dans lequel elles sont implantées, dans une visée de « démocratisation » et de faciliter l'accès à la culture pour les populations. Depuis 2015, la France et plus largement l'Europe, multiplient les initiatives visant à mobiliser ces instances dans l'accueil des populations migrantes. Les très nombreuses propositions doivent aussi faire évoluer les représentations sociales sur les migrations, comme le souligne Gruson, chargé d'examiner «la politique d'accueil des réfugiés mise en place par le gouvernement à partir de septembre 2015, dans sa dimension culturelle mais aussi linguistique, et d'élaborer des préconisations pratiques. » Il engage les acteurs à mener un « travail réciproque [...] vers la société dans son ensemble pour contribuer à changer les regards et combattre les stéréotypes et la xénophobie »(Gruson, 2017 : 173).

Parmi les appels à projets visant à favoriser l'intégration des « migrants » ou des « réfugiés » et à faciliter leur « apprentissage de la langue française », citons l'action lancée en 2015 par le ministère de la Culture et de la Communication qui a largement inspiré Parlemonde :

10. Information disponible sur https://www.scenes-nationales.fr/label/. [Page consultée le 2 octobre 2020.] 
Langue maternelle pour les uns, langue du pays d'accueil pour d'autres, la langue française permet la communication entre tous, sa maitrise étant une composante essentielle du vivre ensemble. [...] Il est donc nécessaire, dans une perspective de réduction des inégalités face à la langue française, d'en faciliter l'accès pour toutes les personnes chez qui les mécanismes de relégation comme de non-participation à la vie sociale produisent une insécurité linguistique, qui est souvent culturelle. Qu'il s'agisse de développer les compétences à l'écrit ou à l'oral, de personnes scolarisées en France ou de permettre à des migrants allophones de communiquer en français, à l'oral et à l'écrit, les pratiques culturelles et artistiques, de même que le contact avec les œuvres et les documents originaux constituent un levier d'action efficace pour l'appropriation du français. En retour, celle-ci peut favoriser l'accès à l'offre culturelle. (Ministère de la Culture et de la Communication, $2015: 1$ )

Kneubühler souligne dans son rapport d'évaluation de cette action que « les actions les plus pertinentes [...] sont précisément des actions où, à l'évidence, la coopération entre professionnels de la culture et formateurs linguistiques a été particulièrement bien pensée » (Kneubühler, 2018). On note que 2 discours sont présents : l'un plaçant les pratiques artistiques comme un «levier » d'accès à la langue - à la fois langage, culture et patrimoine -, cela dans un mouvement à sens unique : l'étranger (dont l'accès à la culture serait empêché) apprend notre langue avec ou par les arts, avec des artistes mais accompagnés de «formateurs linguistiques », puis accède à notre culture ; et l'autre discours, rappelé par Gruson, s'appuyant sur les recommandations du Conseil de l'Europe qui pose «la question du rôle de la culture et des arts dans les processus d'accueil et d'intégration des migrants et dans le changement des représentations sur l'immigration » (Gruson, 2017 : 173). Il s'agit ici d'un mouvement de déplacement réciproque dans une visée de dialogue interculturel, inscrit parmi les priorités du COE. Or, selon lui :

Cette approche est rarement revendiquée en France. En particulier, s'agissant des migrants, tout se passe comme si ces populations n'avaient pas de culture et devaient, par l'apprentissage du français notamment, acquérir nos valeurs culturelles. (Gruson, 2017 :173)

Gruson souligne que les institutions culturelles, ayant une mission d'éducation, sont dans une situation complexe reposant sur des traditions d'exclusion de l'altérité. La conception dominante de la langue est celle d'outil, de moyen de communication, mais c'est également un patrimoine culturel à défendre permettant l'accès aux savoirs et aux œuvres produites et exposées par ces institutions culturelles. Ces dernières font l'objet de nombreux débats quant à la nécessité de, selon les termes de Bonniel : «sortir de [leur] auto-centration et d'entrer (plus exactement de revenir) en dialogue avec d'autres mondes sociaux, d'autres réalités institutionnelles, d'autres pratiques professionnelles, des usagers non définis par leur seule adhésion à la foi culturelle » (Bonniel, 2007 : 24). Cela induit des politiques fortes de « démocratisation » et d'accessibilité de l'art et de la culture. Dans le contexte migratoire actuel, elles témoignent d'une volonté de respecter les « droits culturels » des publics, selon cette définition de l'UNESCO « le droit des peuples à leur propre patrimoine culturel, de même que celui de participer au patrimoine culturel de l'humanité» (UNESCO, 2010 : 247). Des initiatives se 
multiplient en direction des «migrants » : offre tarifaire intéressante voire gratuité, propositions de médiations variées, livrets en « français facile » etc. Ces initiatives assimilent les personnes nouvellement arrivées à la fois à un public potentiel à séduire et au groupe des publics « empêchés », expression euphémisante consacrée pour désigner les milieux populaires ou défavorisés. Cette formule de «public empêché » est régulièrement remise en cause par certains chercheurs et professionnels du secteur, comme le souligne Bordeaux:

Dire qu'il n'y a pas de public spécifique, c'est d'abord soutenir qu'il n'y a pas un type d'œuvre d'art ou un type d'artiste prédisposéà s'adresser ä des publics particuliers, quand bien même ceux qui interviennent à l'hôpital, dans une maison d'arrêt, peuvent éventuellement être amenés à concevoir des propositions en fonction du contexte ou des personnes rencontrées. C'est donc refuser tout rapport d'assignation entre œuvres et publics. C'est aussi défendre, préserver, faciliter la possibilité pour chacun de construire une relation autonome, souveraine, à l'œuvre d'art, quelles que soient les spécificités de sa condition. (Bordeaux, 2007: 19)

Pourtant, la représentation sociale du «public empêché » perdure. Le récent rapport commandé par le ministre la Culture à Benjamin Stora intitulé «Culture et migrants », remis en février 2019, en témoigne. On lit notamment que : « l'orientation [des politiques] doit rester liée à l'affirmation d'un accès d'une population empêchée à ses droits culturels » (p. 9); ; il convient de limiter les dispositifs spécifiques et de privilégier une aide à l'accès aux dispositifs de droit commun ou une intégration de ces publics dans les politiques visant les publics éloignés de la culture» (p. 9) ; enfin, au sujet de la qualité artistique des dispositifs mis en place, l'auteur recommande que : « la qualité artistique des interventions peut être un critère mais non la recherche d'une excellence artistique, [...] afin d'éviter une confusion entre des dispositifs à objectifs culturels et des dispositifs à objectif sociaux » (p. 8). Il y aurait donc une partition des arts et de la « qualité artistique » en fonction de la cible. Si la phrase « Les migrants doivent être à la fois considérés comme destinataires de produits culturels mais également comme potentiels créateurs d'une offre culturelle » vient à la fin de cette même page, l'adjectif « potentiel » et le déterminant indéfini « une » relativisent ce propos. Par ailleurs, l'auteur du rapport mentionne que "l'accès le plus rapide possible à l'apprentissage du français ", est la « principale revendication des associations ${ }^{11}$ ", et que cela doit " être pris en compte » par les autorités (p. 8). Il semble que, dans les représentations des acteurs du terrain (« les associations »), la langue précèderait et conditionnerait l'accès à la culture.

C'est dans ce continuum global de l'obstacle et de l'empêchement: barrière de la langue, difficulté et vulnérabilité (linguistique, scolaire ou sociale) des « migrants » reposant sur une vision ethnocentrée et monolingue des institutions, mais également dans un contexte de volonté interinstitutionnelle d'inclusion des populations allophones déplacées qu'intervient Parlemonde. II a été ainsi baptisé pour faire écho d'une part au multilinguisme du territoire (le Pays de Montbéliard, et par

11. Les associations que l'auteur évoque ici sont celles qui font partie des institutions culturelles ou qui sont leurs partenaires privilégiés. 
extension la France, le monde), et d'autre part à une vision plurielle des langues comme vecteur de rencontre interculturelle créatrice. Par paronomase, son nom se réfère aussi à " parlement » selon la proposition de Charlotte Lagrange ${ }^{12}$, membre de l'équipe artistique qui a créé la pièce Sédiments avec des lycéens allophones lors de la première édition du festival.

\section{Créer c'est relier}

Programme de résidences artistiques monté en coopération, Parlemonde fait le pari de la création comme moteur de reliance au sens « activant » que Morin définit ainsi :

La notion de reliance, inventée par le sociologue Marcel Bolle de Bal, comble un vide conceptuel en donnant une nature substantive à ce qui n'était conçu qu'adjectivement et en donnant un caractère actif à ce substantif. «Relié » est passif, « reliant » est participant, « reliance » est activant. (Morin, $2004:$ 239)

En effet, pour ces concepteurs, partager l'acte de création peut permettre à la fois de relier les EANA à une nouvelle langue entrant dans leur vie, à un nouvel environnement social mais plus largement à un nouveau territoire dont l'exploration dépend de l'opportunité et de la richesse des liens sociaux (Pesqueux, 2009) et qu'il s'agit d'habiter ensemble. Déségrégation et accessibilité sont donc au cœur de Parlemonde, inscrivant les partenaires dans une volonté proactive d'inclusion de tous dans la vie culturelle, reprenant les principes du dialogue interculturels tel que décrits en 2008 dans le Livre blanc sur le dialogue interculturel de la COE, ou les Repères pour un dialogue interculturel de Billerey et Hatzfeld, ouvrage publié en 2010 par le ministère de la Culture et de la Communication.

\subsection{Principes de montage du projet partenarial}

Initié lors de l'année scolaire 2016/2017 en coopération entre MA et le Rectorat de l'académie de Besançon, en particulier avec l'équipe du CASNAV, Parlemonde se déroule principalement (mais pas exclusivement) dans des établissements scolaires (écoles, collèges et lycées) et sur le temps scolaire. Les résidences sont comprises entre 60 et 100 heures d'intervention avec les artistes dans les classes. La présence des artistes à l'école n'est pas un fait nouveau et l'équipe de MA dispose même d'un service dédié à ces actions. Cependant, la collaboration école/artiste s'effectue le plus souvent lors de rencontres ponctuelles ou de projets spécifiques qui s'inscrivent dans le cadre d'un cours disciplinaire de la classe ordinaire (arts plastiques, musique ou lettres), d'un atelier artistique ou d'une option (théâtre, danse, musique). Si les collaborations favorisant les pratiques artistiques sont nombreuses et les invitations institutionnelles pour leur développement de plus en plus fréquentes,

12. Nous ne rendons pas anonymes dans cet article les références aux artistes et à leurs œuvres conçues dans le cadre de Parlemonde, ces œurres étant publiques et produites au même titre que le reste de leur travail. Nous rendons anonymes les données que nous avons suscitées, principalement les discours tenus lors des entretiens semi-directifs ou les travaux d'élèves, préparatoires aux œuvres (extraits de copies, textes ou commentaires divers, messages, etc.). 
notamment suite à l'instauration des parcours d'éducation artistique et culturelle (PEAC) $)^{13}$ devant être documenté par chaque élève tout au long de sa scolarité, elles ne sont pas systématiques sur le terrain et n'entrent pas dans les curriculums en tant que telles. Elles sont mêmes parfois vécues comme des injonctions.

Dans le cas qui nous occupe, chaque enseignant s'est porté volontaire pour engager ses élèves dans Parlemonde. La majorité a répondu à l'invitation du CASNAV. Moins de la moitié connaissait les activités de créations participatives de la structure culturelle. Une suite de contacts s'établit dans lesquels sont explorées les envies de chacun, notamment en ce qui concerne la discipline artistique. C'est enfin la structure qui propose les artistes qu'elle engage et produit, après des échanges évoquant le contexte de création, les modalités d'accueil, de contrat et la rédaction d'une « note d'intention » traçant les grandes lignes du projet de chaque artiste. Cette note d'intention est ensuite présentée aux enseignants et éventuellement ajustée en fonction des informations échangées.

\subsection{Enjeux didactiques pour l'école}

La volonté commune des concepteurs de Parlemonde s'appuie sur l'hypothèse de compétences interculturelles et plurilingues importantes (Abdallah-Pretceille, 1999) développées dans un cadre de mobilité ou migration (Moro, 2002, 2010, 2015) et sur l'hypothèse du projet artistique public comme booster de l'apprentissage de la langue française cible (Pierra 2006 ; Schmidt 2006 ; Auger, 2007 ; Clerc et al 2007 ; Aden, 2009, 2013, 2016 ; Eschenauer, 2018). Les équipes du CASNAV, incluant les enseignants des dispositifs UPE2A dans lesquels est scolarisé l'essentiel des participants de Parlemonde, parient sur le fait que les processus et dynamiques de co-création engagés lors des périodes de résidence des artistes aux côtés des EANA transformeraient leurs pratiques (quel que soit leur champ professionnel) et valoriseraient simultanément les compétences des EANA aux yeux de tous (élèves, parents, équipes éducatives et artistiques, public).

Cette conception est également nourrie par les recherches en sciences de l'éducation qui ont montré que la pédagogie de projet accélérait, voire révélait, les compétences des élèves alors même qu'elles étaient invisibles ou invisibilisées par des tâches scolaires ordinaires. Pourtant, monter un tel projet sur le temps de l'école et malgré le soutien du Rectorat ne va pas de soi. Chrifi-Alaoui écrivait en 2009 que « le projet artistique appartient à une catégorie particulière. L'art est un terme et même un domaine qui fait encore peur. On veut l'éloigner de l'école par crainte du désordre ou pire par craindre de perdre son temps » (p.27). En classe ordinaire en effet, les pratiques artistiques restent à la marge, le sont de plus en plus à mesure que les examens s'approchent et où les exigences scolaires s'accroissent. S'il persiste des activités dites artistiques, elles sont le plus souvent ponctuelles et leur finalité est d'accomplir une tâche mobilisant la « créativité » des élèves (conception d'une exposition, présentation aux parents ou à des pairs de textes écrits en cours de français puis joués, restitution de

13. Information disponible sur http://eduscol.education.fr/cid74945/le-parcours-education-artistique-culturelle. html. [Page consultée le 2 octobre 2020.] 
la chorale, etc.). Elles sont le plus souvent conduites sans artiste et prétextes à une co-intervention entre enseignants de disciplines différentes. Plus fréquentes dans le champ de l'enseignement adapté ou spécialisé (dispositifs de lutte contre le décrochage scolaire ou d'accueil des élèves en difficulté ou en situation de handicap), elles s'inscrivent alors dans une démarche de «pédagogie du détour » et sont envisagées comme un vecteur de remobilisation des élèves en difficulté d'apprentissage, ce que Henri-Panabière et al. nomment la pédagogie du « détour pour un retour ». De plus, un pouvoir de « réparation symbolique » agissant sur la personnalité des élèves concernés (Bonnéry et Renard, 2013 : 144) leur est souvent attribué.

\subsection{Enjeux pour la structure artistique}

Pour la scène nationale, Parlemonde est une première dans un tel contexte sociétal et avec de tels participants. Bien que familière des projets de création participative en milieu scolaire, MA connait avec Parlemonde sa première expérience de coopération d'ampleur qui dépassera la durée d'une saison et deviendra un festival en biennale qui connaitra en 2021 sa $3^{\text {ème }}$ édition. C'est donc un investissement important tant dans l'implication des équipes artistiques et techniques que financièrement puisque la structure culturelle supporte l'essentiel des coûts. Voici un tableau récapitulatif qui présente les œuvres créées ${ }^{14}$ en 2017 et 2019 avec 250 participants et une quinzaine d'artistes européens:

Tableau 1 : Récapitulatif des créations Parlemonde

\begin{tabular}{|c|c|c|c|c|}
\hline \multirow{4}{*}{ Parlemonde 1 } & Artistes & Titres & Disciplines & Participants \\
\cline { 2 - 5 } & Sébastien Fayard & (Dé)formations & Photographie & CM2 \\
\cline { 2 - 5 } Mai 2017 & Thomas Boichard & Déviation & Musique & $\begin{array}{c}\text { MNA (hors temps } \\
\text { scolaire) }\end{array}$ \\
\cline { 2 - 5 } & David Subal & $\begin{array}{c}\text { Orientations (Un par- } \\
\text { tage des chemins) }\end{array}$ & $\begin{array}{c}\text { Installation, exposi- } \\
\text { tion et performance }\end{array}$ & UPE2A lycée \\
\cline { 2 - 5 } & Wil Mathijs & Becoming & Vidéo & UPE2A collège \\
\cline { 2 - 5 } & Frédéric Dumond & $\begin{array}{c}\text { Le Bruissement des } \\
\text { langues }\end{array}$ & $\begin{array}{c}\text { Poésie et perfor- } \\
\text { mance }\end{array}$ & UPE2A lycée \\
\cline { 2 - 5 } & Charlotte Lagrange & Sédiments & Théâtre & UPE2A lycée \\
\hline
\end{tabular}

14. D’autres éléments ainsi que des images et/ou vidéos sont disponibles sur le site dédié en cours d'actualisation: http://parlemonde.mascenenationale-creative.com 


\begin{tabular}{|c|c|c|c|c|}
\hline \multirow{4}{*}{ Parlemonde 2 } & $\begin{array}{c}\text { Cédric Charron \& } \\
\text { Annabelle Chambon }\end{array}$ & $\begin{array}{c}\text { Service d'Action Cho- } \\
\text { régraphique }\end{array}$ & Danse/ Performance & $\begin{array}{c}\text { Tout public (hors } \\
\text { temps scolaire) }\end{array}$ \\
\cline { 2 - 5 } Mai 2019 & Thomas Boitage & $\begin{array}{c}\text { Musique et arts plas- } \\
\text { tiques }\end{array}$ & $\begin{array}{c}\text { CM2 + CM2 et CE2 } \\
\text { pour le volet illustra- } \\
\text { tion graphique }\end{array}$ \\
\cline { 2 - 5 } & $\begin{array}{c}\text { David Subal \& Clara } \\
\text { Cornil }\end{array}$ & $\begin{array}{c}\text { D'ailleurs \& Carte } \\
\text { d'identités }\end{array}$ & $\begin{array}{c}\text { Installation, exposi- } \\
\text { tion et performance }\end{array}$ & $\begin{array}{c}\text { UPE2A Cité scolaire } \\
\text { (collège et lycée) }\end{array}$ \\
\cline { 2 - 5 } & Jorge Picó & Sauver les mots & Théâtre & UPE2A collège \\
\cline { 2 - 5 } & $\begin{array}{c}\text { Renaud Diligent \& } \\
\text { Maya Boquet }\end{array}$ & $\begin{array}{c}\text { Un Banquet } \\
\text { Cédric Orain }\end{array}$ & $\begin{array}{c}\text { Bibliothèque Hu- } \\
\text { maine Internationale } \\
\text { (BHI) }\end{array}$ & $\begin{array}{c}\text { UPE2A lycée + } \\
\text { classe de CAP }\end{array}$ \\
\cline { 2 - 5 } & $\begin{array}{c}\text { Écriture/ } \\
\text { performance }\end{array}$ & $\begin{array}{c}\text { Tout public (hors } \\
\text { temps scolaire) }\end{array}$ \\
\hline
\end{tabular}

D’un point de vue méthodologique, les artistes ont été formés par le CASNAV en amont des résidences sur la question des plurilinguismes et de l'allophonie. Ils sont libres d'engager leur résidence dans la (ou les) langue(s) qu'ils souhaitaient, tous ne sont pas des francophones natifs mais tous sont plurilingues comme le montre le tableau suivant:

Tableau 2 : Répertoires linguistiques des artistes ayant travaillé en milieu scolaire

\begin{tabular}{|c|c|c|}
\hline & Langues premières & $\begin{array}{c}\text { Autres langues (par ordre de com- } \\
\text { pétence estimée) }\end{array}$ \\
\hline Artiste 1 & Français & Allemand, anglais \\
\hline Artiste 2 & Français & Anglais, espagnol \\
\hline Artiste 3 & Néerlandais flamand & Anglais (LS), français \\
\hline Artiste 4 & Français & Anglais, espagnol \\
\hline Artiste 5 & Français & $\begin{array}{l}\text { Latin, grec ancien, anglais, grec } \\
\text { moderne }\end{array}$ \\
\hline Artiste 6 & Allemand (Autriche) & Anglais (LS), français, latin \\
\hline Artiste 7 & Catalan, espagnol & Anglais, français \\
\hline Artiste 8 & Français & Allemand, anglais \\
\hline
\end{tabular}

Le choix des langues de travail s'est fait le plus souvent spontanément et principalement en fonction des interactions avec les participants. Les artistes de langue maternelle française les ont conduites en français exclusivement, les autres ont utilisé toutes les langues de leur répertoire, souvent pour traduire ou développer une consigne.

Le projet artistique est resté un espace de création donc peu contraint. Deux points de vigilance ont cependant été abordés dès les premiers échanges entre les différents partenaires : permettre aux participants de s'exprimer dans les langues de leur choix, et cela sans assignation particulière et avec la possibilité d'en changer à tout moment ; évacuer les entrées artistiques reposant purement sur des considérations biographiques de type «témoignages » ou documentaire visant à faire partager 
les parcours de migrations et autres traumatismes vécus au cours de celle-ci au public, écueil parfois présent dans la création théâtrale contemporaine (Carré, 2017).

\subsection{Place et profils linguistiques des élèves participants}

Les participants ont co-écrit, monté, joué les œuvres dont le processus créatif avait été imaginé par les artistes dans leur note d'intention. En cela, les élèves ont été co-auteurs, co-créateurs à des degrés divers de participation selon les sensibilités de chacun, mais ils n’ont pas mis en scène ou chanté ou exposé de textes pré-écrits ou pré-imaginés pour eux. Ils portent leur parole, négociée, travaillée collectivement et individuellement, dont les fonctions esthétiques et symboliques sont à la fois conscientisées et valorisées. Cette parole s'exprime dans de nombreuses langues. Pour en avoir une idée, voici les langues familiales déclarées par participants des résidences scolaires suivies ${ }^{15}$, sachant qu'elles composent mais ne reflètent pas de manière exhaustive les répertoires linguistiques des élèves. Sur les 167 élèves documentés : 90 déclarent au moins deux langues familiales. On dénombre 37 langues déclarées (mais 35 distinctes) et 1 participant dont la langue première n'est pas renseignée ; 10 participants pour lesquels le français est cité en premier dans les langues familiales, 26 pour lesquels le français est cité comme seconde langue. Aucun des 167 élèves interrogés ne se définit comme monolingue (y compris les locuteurs natifs de France).

Tableau 3 : Langues familiales déclarées par les élèves

\begin{tabular}{|c|c|c|c|}
\hline Langues familiales & Nombre de locuteurs & Langues familiales & Nombre de locuteurs \\
\hline Arabe & 68 & Ourdou & 3 \\
\hline Français & 36 & Malinké & 2 \\
\hline Italien & 21 & Dioula & 2 \\
\hline Albanais & 14 & Swahili & 2 \\
\hline Espagnol & 12 & Somali & 2 \\
\hline Portugais & 10 & Sylheti & 1 \\
\hline Bambara & 8 & Wolof & 1 \\
\hline Lingala & 7 & Amharique & 1 \\
\hline Turc & 7 & Baoulé & 1 \\
\hline Serbo-croate & 7 & Kimbundu & 1 \\
\hline Peul & 5 & Kirghize & 1 \\
\hline Shimaoré & 5 & Mandarin & 1 \\
\hline Bengali & 4 & Soussou & 1 \\
\hline Soninké & 4 & Tchèque & 1 \\
\hline Persan & 3 & Tzigane & \\
\hline Kabyle & 3 & Diakhanké & \\
\hline
\end{tabular}

15. Sur les 251 participants au total, les chiffres présentés ici concernent les élèves du secondaire (collège et lycée) soit 167 élèves ayant participé à 7 projets. 


\begin{tabular}{|c|c|c|c|}
\hline Langues familiales & Nombre de locuteurs & Langues familiales & Nombre de locuteurs \\
\hline Russe & 3 & Non référencé & 1 \\
\hline Rif & 3 & & \\
\hline
\end{tabular}

\section{Les pratiques artistiques : du détour au passe-murailles}

Le recours aux pratiques artistiques pour remédier à une situation particulière ou pour maintenir la « cohésion sociale » renvoie à l'idée que l'art, selon la formule de Pignot (2007) serait « passe-muraille » et permettrait de renverser, voire de soigner les fractures sociales de nos sociétés. Dans notre cas, les arts ont surtout permis de faire œuvre et de faire entendre la voix des élèves au sens propre comme au figuré, favorisant leur inclusion sociale.

\section{1. Évolution des représentations à propos des langues des participants}

La variété des langues et des contextes de leur utilisation a suscité nombre d'échanges et d'interrogations, tant du côté des artistes, peu habitués à co-créer avec des jeunes allophones (2 en avaient l'expérience), que du côté des participants, peu habitués à travailler avec des artistes, encore moins dans un cadre scolaire, et surpris d'être invités à dévoiler, et à utiliser, leurs langue(s) première(s). Nous ne développerons pas ici les étapes du processus de création ni celles qui ont conduit, le cas échéant, au changement de point de vue quant aux langues des participants. Cependant nous pouvons évoquer les moments de médiation que nous avons conduits afin de répondre à certaines questions des artistes ou d'en faire germer d'autres. Cette fonction de médiation, initialement imprévue (Sérusclat-Natale et Marzin, 2020) a été très importante pour conscientiser, auprès des acteurs concernés par l'expérience esthétique (participants au sens large et publics), les représentations qu'ils avaient des langues et des cultures, puis d'explorer leurs propres origines.

\subsubsection{Pour les artistes}

Aucun artiste n'a demandé comment communiquer avec les élèves, mais tous se sont questionnés sur les possibilités d'accès à l'écrit. On a constaté dans les entretiens de fin de projet menés avec eux que le niveau de compétences en français des élèves était généralement oublié, comme en témoigne artiste 8 ici :

D : Avais-tu déjà travaillé avec les élèves allophones?

Artiste 8 : J'ai déjà travaillé dans des conditions compliquées avec des jeunes... heu... tu vois, de... issus de... de tu vois... en galère sociale et en rupture scolaire et enfin voilà dans des conditions compliquées heu... voilà... mais ce type de public avec heu différentes langues... mais en même temps ils parlaient beaucoup, tous français, hein quand même pour la plupart, il... il y avait quand même peu de vraiment de non... enfin allophones à $100 \%$ et heu... 
Or, dans cette résidence effectuée avec 15 élèves de l'UPE2A, 7 élèves ne s'exprimaient pas du tout en français au démarrage du projet. L'artiste n'en garde pas le souvenir mais assimile les EANA aux publics « empêchés » avec lesquels il a déjà travaillé auparavant. Pour certains artistes, le plurilinguisme des participants les attirait parce que le sujet était actuel (le plurilinguisme étant associé au thème des migrants à leurs yeux, l'un a d'ailleurs employé l'expression « me frotter à ça ») pour d'autres c'était un défi à relever dans le sens d'un critère supplémentaire d'« excellence » du projet. Au fil des résidences, les questions se sont portées plus largement sur comment ils pouvaient accéder à l'imaginaire des jeunes, à leurs regards sur le monde et à ce qu'ils avaient envie d'exprimer. Il ne s'agissait plus de communiquer mais plutôt de s'exprimer. Les langues employées n'étaient pas un obstacle, le cadre scolaire l'a été, comme on le voit dans les extraits d'entretien suivants :

\section{Entretien semi-directif avec Artiste 5 post Parlemonde 1}

D: Vois-tu une/ des différences entre les publics scolaires avec lesquels tu as déjà travaillé et les élèves allophones de cette année?

Artiste 5 : Je ne vois pas tant de différence. À part... non non vraiment j'en ai pas vu. À part cette histoire d'efficacité plutôt que dans la création, la question de la rentabilité [...] Une grosse exigence scolaire chez certains, chez d'autres moins. Plus largement, plus d'exigence scolaire qu'avec les autres publics avec qui j'ai travaillé. [...]

D : Tu pourrais décrire les participants?

Artiste 5 : Volontaires, prêts à expérimenter tout sans comprendre forcément - quels étaient les enjeux je veux dire - mais dans le désir, le plaisir de voir où ça menait. Des doutes, forcément mais émis ou gardés avec discrétion, mais qui se font sentir, c'est normal. Une vraie volonté, enfin l'histoire c'est qu'on y va quoi! Quel que soit ce qu'on leur propose, ils y vont quoi ! Il y aurait pratiquement plus de volonté chez eux que dans les groupes ordinaires.

\section{Entretien semi-directif avec Artiste 7 post Parlemonde 2}

Artiste 7 : Au débutj'ai pensé « ils ne peuvent pas » parce qu'il y avait certains conflits entre eux qui disaient qu'ils n'étaient pas dans des conditions de faire du théâtre, ils ne formaient pas un groupe tu vois, et ils étaient petits. À mon avis le processus ils ont grandi pendant les répétitions et ils ont compris que... il faut dépasser ça tu vois. Au-delà de ça, c'est des gens qui ont un vécu personnel, le fait de devoir s'exprimer dans une langue et puis d'un coup dans une autre heu, ça les rend plus forts tu vois. Mais au début effectivement je me suis dit « ça va être trop quoi », parce que... ils ont déjà heu... déjà la situation un peu spéciale mis de côté un peu ghetto quand même, ça fait que entre eux, ils ont des problèmes pour s'accepter comme un groupe, pour s'aimer comme un groupe, et mais une fois que j'ai compris qu'ils pouvaient dépasser cela... ils comprennent quoi. Ils comprennent. Ils comprennent bien quoi [...] Mais heu... au début on a tous peur hein, même le metteur en scène hein... On va y pas arriver, on 
ne sait pas trop où on va aller. [...] C'est vrai qu'humainement le groupe avec les allophones c'est le plus fort quoi.

D : Humainement? Pourquoi tu dis ça?

Artiste 7 : au niveau des réactions, des palpitations, des demandes, [...] ça palpitait plus que dans les autres groupes, et ça c'est très beau. C'est très important. Parce que si ça palpite ça veut dire c'est vivant derrière, y a des vrais conflits des vraies passions, le théâtre il est fait de ça tu vois. Dans les autres groupes il y a eu moins ça; il y a eu beaucoup moins ça.

Ces extraits montrent que l'allophonie et le plurilinguisme, initialement considérés comme un sujet auquel il faudra «se frotter », ont fait écran. La difficulté ressentie venait plutôt à la position de vulnérabilité de l'artiste lui-même au démarrage d'une création. Cette vulnérabilité s'efface dans et par le travail artistique et ce processus révèle d'autres obstacles plus systémiques comme la peur du désordre du côté des enseignants et le souci de rentabilité du temps scolaire.

À ce sujet, Artiste 7 déclare :

Même si après on a besoin des règles de cadrer tout ça oui, mais quand même, certains profs n’ont pas profité du désordre. Le théâtre est lié au désordre. Le désordre c'est très important, le chaos s'il évolue, s'il est aimé, il est très important dans un processus d'éducation, c'est une métaphore de la vie, la vie on ne la contrôle pas tout le temps. Il faut décontroler ${ }^{16}$ les groupes.

\subsubsection{Pour les élèves}

Nous appuyons notre propos sur les mots utilisés par les participants dans les questionnaires de fin de projet, ou dans les entretiens semi-directifs ou dans nos notes de terrain. Pour l'écrasante majorité des élèves participants à Parlemonde, ce festival était la première occasion de collaborer avec un artiste. Certains ont éprouvé quelques difficultés à s'adapter à ces activités artistiques dans la mesure où elles ne faisaient pas partie de leurs représentations de l'école « sérieuse », et du travail scolaire « pour progresser ». Cela a particulièrement été le cas des lycéens, soumis à une forte pression scolaire. On peut d'ailleurs se demander si ces représentations des pratiques artistiques comme « loisirs » sont les leurs, ou si elles témoignent d'une intériorisation des représentations de certains enseignants ou adultes considérant les arts comme une récompense divertissante. Armagnague et al., en 2019, parlent d'une «scolarité loisirs » en abordant ces pédagogies, ce qui montre que cette représentation des pratiques artistiques demeure. Il a donc fallu effectuer ponctuellement des médiations pour encourager les jeunes à poursuivre le projet et les rassurer sur les modalités de rattrapage ou les dispenses des cours manqués. Ces médiations, parfois devant désamorcer des tensions, ont été saluées en fin de projet par les lycéens qui déclarent s'être sentis « entre adultes » (faisant référence à l'horizontalité des relations qui s'était installée par les pratiques artistiques et à la liberté

16. Artiste 7 est hispanophone. En espagnol « descontrolar » est un verbe qui veut dire « perdre le contrôle». 
de s'exprimer). Ils ont également très souvent relevé qu'ils avaient fortement apprécié « être encouragés » par les enseignants, les artistes et les équipes de production, ce qui les a rendus « forts ». Enfin, concernant l'appropriation de la langue française, les EANA disent qu'ils ont progressé, notamment à l'oral : « J'ai appris le français, j'ai appris que j'étais capable. » Pour quelques-uns, Parlemonde a été l'occasion de s'exprimer en français pour la première fois, profitant d'un effet de groupe et d'un effort particulier fait « pour l'artiste » ou « pour notre troupe ». Ils déclarent par exemple : « Oui ça m’a aidée car tout le monde a parlé français donc pour parler avec eux on parle aussi français »; " Avant je ne savais pas m'exprimer mais après le spectacle je m'exprime et je reste plus dans mon coin toute seule. Maintenant je parle plus avec les gens et je suis moins timide. » L'envie de «se lancer » vient aussi de l'approche poétique de la langue comme moyen d'expressivité subjective et non plus comme instrument de communication scolaire, comme l'exprime cet élève : "Dans le projet on a mélangé les langues de tous et je trouve que tout se complète. Un peu. J'ai aimé parler bengali et anglais. Mais maintenant j'aime beaucoup le français et je ne parle plus anglais alors que c'est la langue que tout le monde veut me parler. Aujourd'hui, je me lance dans le français. Je travaille mon accent, le français c'est une langue comme de la musique, ça ressemble à la musique des oiseaux, c'est rapide et dans tous les sens. »

Certains relient leur implication dans Parlemonde et leurs bons résultats au DELF. Pourtant, la majorité des participants déclare que faire partie du projet ne les a pas particulièrement aidés dans leur «métier d'élève ». Si la « rentabilité scolaire » est une inquiétude pendant le temps de résidence et parce que la création s'inscrit dans un temps long, non séquencé comme le temps scolaire et plus opaque (parfois on ne sait pas où l'on va), après le projet, les élèves n'expriment pas d'insatisfaction particulière ni n'affirment avoir perdu leur temps. Au contraire, tous (sauf 2 élèves) voudraient recommencer. Alors que j'interrogeais un groupe de lycéens sur ce que je percevais comme un paradoxe (vouloir recommencer alors que cela ne leur a pas « servi » dans leur métier d'élève), l'une d'entre eux, après quelques secondes de silence, m’a répondu : «Aujourd'hui, maintenant, quand je marche : j’ai la tête haute. » Cette élève n'est pas un cas isolé. Les propos relatifs à la hausse de l'estime de soi et au sentiment d'être ou de se sentir (enfin) capable, ont été nombreux et les élèves associent, dans leurs réponses, ce sentiment de mieux-être à leur réussite scolaire.

\subsection{L'importance de l'exposition publique}

Nous n'aborderons pas ici les traces de réception par le public mais plutôt les propos des élèves participants au sujet du temps des représentations publiques. Aboutissement valorisant du travail engagé, le temps du festival est un temps fort pour tous. C'est aussi le point culminant du stress ressenti et de l'envie de bien faire, et le dernier déclencheur du passage en français pour de nombreux participants. L'un d'eux, âgé de 12 ans, l'exprime ainsi : « Finalement j'ai pas utilisé mon arabe. Mme $X$ m'a laissé le choix mais je n'avais pas envie de passer mon temps à traduire, j'ai préféré qu'on m'entende en français. » Une autre, lycéenne, a choisi de traduire en français ses propos enregistrés en arabe lors de la résidence et de proposer les deux langues au public avançant: « Je pense que le 
public il va parler français alors je veux être sure qu'il m'écoute, j'ai des choses à lui dire. » Il s'agit pour eux d'adresser une parole, non pas seulement de parler ou d'être visibles dans un travail créatif dont ils n'auraient pas élaboré le message et le sens. Ainsi, pour que les expériences artistiques soient profitables, la co-création et l'exposition publique incluant des interactions sont deux aspects majeurs. Dans ce travail commun s'est installée une relation et de la qualité de celle-ci, de son intensité, dépend l'œuvre finale et sa présentation publique. Cela rappelle les termes de Moro :

C'est le processus social, culturel, identitaire, dans lequel sont [les EANA] qui est vulnérabilité mais il peut devenir créativité au sens où on peut faire quelque chose avec ces différences à partir du moment où on les reconnait, on les inscrit, on les valorise, où elles ne restent pas hors cadre, hors école. (Moro, 2017)

On peut aller plus loin et dire qu'elles ne doivent pas rester hors cité, et que l'aspiration existentielle selon les mots du poète Francis Ponge, le « je parle et tu m'entends donc nous sommes » est très importante pour les EANA. Selon Artiste 7, ce sentiment procède du travail artistique : "Qui je suis?" demande le théâtre, le théâtre te fait dire "je suis par rapport à quelqu'un". On a pas mal creusé le "qui je suis" avec ce groupe, mon essence, l'essence, mais ton essence c'est le... le rapport à l'autre, face au visage de l'autre et aux mots de l'autre. »

Parmi les performances réalisées par les élèves, 2 ont été faites en interaction directe avec le public ${ }^{17}$, soit sous la forme de "face à face ", soit de performances chez l'habitant. Summum de l'exposition au sens où les participants sont, d'une part, amenés à suivre un protocole artistique abolissant les distances, tant physiques que psychologiques, avec un public d'inconnus, et d'autre part à improviser en fonction des réactions de l'auditoire. Ces projets sont ceux qui ont été préférés par les participants, décrits par de nombreuses expressions superlatives comme une " aventure fantastique », « forte », « incroyable ». Un lycéen explique : « C'est vraiment la prise de risque qui m’a plu, c'est pour ça que je l'ai préféré. »

\section{Conclusion}

Tenter l'expérience de la co-création artistique avec les EANA ne va pas de soi et ne constitue pas un remède miracle aux situations de vulnérabilité rencontrées par les élèves et les enseignants, souvent érigées par le système scolaire lui-même. En revanche, lorsqu'elles sont authentiquement participatives, elles permettent d'inscrire les apprenants dans une relation fondée sur l'intersubjectivité qui autorise à la fois les recours à toutes les langues des répertoires, mais également les erreurs, les essais, en un mot : le risque, comme le synthétise Coïaniz:

17. Il s'agissait du travail mené avec l'artiste David Subal en 2017 et en 2019. Voir http://parlemonde.mascenenationale-creative.com 
Un apprentissage quel qu'il soit (et à bien des égards, plus encore le langage, en cela qu'il vient profondément reconstruire nos repères et notre vision du monde) apparait comme une conquête, un risque (pour l'image de soi, la place qu'on occupe la cohésion parfois difficilement tenue entre nos pratiques, ce qu'on en ressent, ce qu'on en dit et ce que l'on se représente), conquêtes et risques toujours interactionnels donc toujours sociaux. (Coïaniz, 2001 : 120)

Parler et se dire dans une autre langue ne requiert pas qu'enseignement de la grammaire ou du lexique d'usage. Cela requiert avant tout audace, imagination, flexibilité et désir, qualités très présentes chez les EANA que nous avons suivis, et que rend visibles la pratique artistique. Souvent perçues comme inutiles ou peu rentables scolairement, ou au contraire thérapeutiques et vectrices de « réparation symbolique », les expériences de collaboration artistiques ne peuvent s'improviser et se passer de médiations expertes. Elles s'inventent en équipe. Enfin, elles renforcent, par un mouvement de « décentration des habitus » (Pierra, 2006) dont chaque participant (enfants comme adultes, acteurs comme spectateurs) fait l'expérience, et rendent, à la fois possibles et publiques, une reconnaissance sensible et partagée des langues et des cultures qui font l'identité d'un territoire. 


\section{Références}

Abdallah-Pretceille, Martine (1999), L'éducation interculturelle, Paris, Presses Universitaires de France.

Adam-Maillet, Maryse et Maud Sérusclat-Natale (2019), "L'hospitalité, l'école et le sacré, la question des mineurs non accompagnés », Diversité, nº 196 (L’hospitalité n², sous la dir. de Régis Guyon), p. 126-132.

Aden, Joëlle (dir.) (2008), Apprentissage des langues et pratiques artistiques : créativité, expérience esthétique et imaginaire, Paris, Le Manuscrit Universitê.

Aden, Joëlle (2013), « Apprendre les langues par corps », dans Pour un Théâtre-Monde: plurilinguisme, interculturalitéet transmission, Bordeaux, Presses Universitaires de Bordeaux.

Aden, Joëlle (2016), « Créer, innover par le théâtre : pour une pédagogie énactive des langues », dans Isabelle Capron Puozzo (dir.), La créativité en éducation et en formation : perspectives théoriques et pratiques, Louvain-la-Neuve, De Boeck, p. 107-118.

Armagnague, Maïténa, Alexandra Clavé-Mercier, Marion Lièvre et Anne-Claudine Oller (2019), "Les dispositifs de scolarisation des enfants et jeunes migrants en France: des producteurs de "scolarités contraintes" », Cahiers de la recherche sur l'éducation et les savoirs, nº 18, 147-172.

Armagnague, Maïténa, Isabelle Rigoni, Claire Cossée, Catherine Mendonça Dias et Simona Tersigni (2018), Étude sur la scolarisation des élèves allophones nouvellement arrivés (EANA) et des enfants issus de familles itinérantes et de voyageurs (EFIV), Rapport de recherche EVASCOL, disponible sur https://www.defenseurdesdroits.fr/fr/etudes-et-recherches/2018/12/etude-sur-la-scolarisation-des-eleves-allophones-nouvellement-arrives. [Page consultée le 17 août 2020.]

Auger, Nathalie, Olivier Terrades et Richard Talagrand (2007), «Ateliers littérature et arts pour entrer dans le français langue seconde », ELA: Études de linguistique appliquée, vol. 147, n³ 3, p. 339-348.

Auger, Nathalie (2008), "Le rôle des représentations dans l'intégration scolaire des enfants allophones » dans Jean-Louis Chiss (dir.), Immigration, École et didactique du français, Paris, Didier, p.187-230.

Auger, Nathalie (2010), Élèves nouvellement arrivés en classe : réalités et perspectives, Paris, Éditions des archives contemporaines.

Auger, Nathalie (2013), «Continuum/clivage de représentations dans les définitions de la difficulté scolaire en matière de langue(s) », dans Stéphanie Galligani, Sandrine Wachs et Corinne Weber (dir.), École et langues: des difficultés en contextes, Paris, Riveneuve, p. 119-138.

Blanchet, Philippe (2016), Discriminations : combattre la glottophobie, Paris, Textuel.

Billiez, Jacqueline (1985), «La langue comme marqueur d’identité », Revue européenne des migrations internationales, vol. 1, n², p. 95-105.

Bonnéry, Stéphane et Fanny Renard (2013), « Des pratiques culturelles contre l'échec et le décrochage scolaires : sociologie d'un détour », Lien social et Politiques, n 70, p. 135-150. 
Bonniel, Jacques (2007), «Élargir le cercle des connaisseurs », L'Observatoire : la revue des politiques culturelles, n 32, p. 23-27.

Bordeaux, Marie-Christine (2007), "Il n’y a pas de public spécifique », L'Observatoire : la revue des politiques culturelles, n³2, p.19.

Calvès, Anne-Emmanuèle (2009), « "Empowerment” : généalogie d’un concept clë du contemporain sur le développement », Tiers Monde, vol. 200, nº 4, p. 735-749.

Calvet, Louis-Jean (1974), Linguistique et colonialisme: petittraité de glottophagie, Paris, Payot. Carré, Alice (2017), « Récits d'exil en scène », E-Migrinter, n 16, disponible sur http://journals.openedition.org/e-migrinter/941. [Page consultée le 16 août 2020.]

Chrifi-Alaoui, Dalie (2009), «Apprendre le français par l'oblique », Cahiers pédagogiques, n 473, p. 2628.

Clerc, Stéphanie, Claude Cortier, Amandine Longeac et Gérard Oustric (2007), «Place et intérêt des pratiques artistiques, interculturelles dans les dispositifs d'accueil et d'enseignement/apprentissage pour les élèves allophones », ELA: Études de linguistique appliquée, vol. 147, n³, p. 317-328.

Coïaniz, Alain (2001), Apprentissage des langues et subjectivité, Paris, L'Harmattan.

Cummins, Jim (2001), Negociating identities : Education for empowerment in a diverse society, $2^{e}$ éd., Los Angeles, California association for bilingual education. [1e éd., 1996.]

Ebersold, Serge et Maïtena Armagnague-Roucher (2017), «Importunité scolaire, orchestration de l'accessibilité et inégalités », Éducation et sociétés, n³9, p.137-152.

Ennuyer, Bernard (2017), «La vulnérabilité en question », Ethics, Medicine and Public Health, n³, p. 365-373.

Eschenauer, Sandrine (2018), "Créativitê et empathie dans les apprentissages performatifs : vivre et encorporer ses langues », Recherches \& Éducation, hors-série, disponible sur : http://journals.

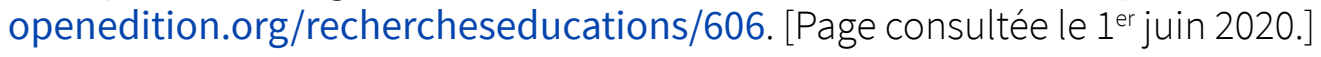

Escudé, Pierre (dir.) (2018), Langues et discriminations, numéro thématique des Cahiers de la LCD, $n^{\circ}$ 7, Paris, L'Harmattan.

García, Ofelia (2009), «Emergent bilingual and TESOL: What's in a name? », TESOL Quaterly, vol. 43, no 2, p. 322-326.

Gavini-Chevet, Christin, Yves Delécluse et Christian Bigaut (2018), Les aménagements d'épreuves d'examens pour les élèves et étudiants en situation de handicap, rapport de l'Inspection générale de l'administration de l'Éducation nationale et de la Recherche, disponible sur https://www. education.gouv.fr. [Page consultée le 2 octobre 2020.]

Goï, Cécile (2015), « Langues et rencontre interculturelle en éducation : loyautés, conflits, autorisations » dans Accueillir l'enfant et ses langues : rencontres pluridisciplinaires sur le terrain de l'école, Paris, Riveneuve. 
Gruson, Luc (2017), «Comment mettre la culture au service des migrants? » Hommes et migrations, vol. $1, n^{\circ} 1316$, p. 170-179.

Henri-Panabière Gaële, Fanny Renard et Daniel Thin (2013), « Des détours pour un retour? Pratiques pédagogiques et socialisatrices en ateliers relais », Revue française de pédagogie, n 183, p. 71-82.

Kneubühler, Michel (2018), Détours \& déclics : action culturelle et langue française, Paris, La passe du vent.

Lüdi, Georges et Bernard Py (1986), Être bilingue, Berne, Peter Lang.

Mestre, Claire (2015), "Parentalité, migration et exil, comment prendre soin des parents ? », Spirale, n०73, p. 206-216.

Métraux, Jean-Claude (2011), La migration comme métaphore, Paris, La Dispute.

Ministère de la Culture et de la Communication (2015), Appel à projets national : "L'action culturelle au service du français », disponible sur https://aaar.fr/wp-content/uploads/2015/06/Appel-aprojets.pdf. [Page consultée le 2 octobre 2020.]

Morin, Edgar (2004), La Méthode, vol. 6, Paris, Seuil.

Moro, Marie-Rose (2002), Enfants d'ici venus d'ailleurs, Paris, Hachette Littératures.

Moro, Marie-Rose (2010), Grandir en situation transculturelle, Paris, Fabert.

Moro, Marie-Rose (dir.) (2015), L'adolescent créatifface aux malaises de la société, Paris, Armand Colin.

Moro, Marie-Rose (2017), Pilotage des dispositifs d'accueil et de scolarisation des élèves allophones nouvellement arrivés: quels parcours d'inclusion pour des élèves en situation de vulnérabilité ?, discours prononcé dans le cadre du Plan national de formation d’Éduscol, disponible sur https://www.canal-u.tv/video/eduscol/intervention_de_marie_rose_pilotage_des_dispositifs_d_accueil_et_de_scolarisation_des_eleves_allophones.3679. [Page consultée le 2 octobre 2020.]

Organisation de coopération et de développement économiques (OCDE) (2008), Élèves présentant des déficiences, des difficultés et des désavantages sociaux, disponible sur http://www.oecd. org/fr/education/scolaire/41058722.pdf. [Page consultée le 2 octobre 2020.]

Organisation mondiale des Nations unies pour l'éducation, la science et la culture (UNESCO) (2010), Investir dans la diversitéc culturelle et le dialogue interculturel : rapport mondial de l'UNESCO, Paris, Éditions UNESCO.

Pesqueux, Yvon (2009), «La notion de territoire », communication présentée au colloque Observatoire économique des banlieues, Propedia, Paris, décembre 2009, disponible sur : https://hal.archives-ouvertes.fr/hal-00479794/document. [Page consultée le 7 novembre 2020.]

Pierra, Gisèle (2006), Le corps, la voix, le texte, arts du langage en langue étrangère, Paris, L'Harmattan. 
Pignot, Lisa (2007), «L'art comme passe-muraille », L'Observatoire : la revue des politiques culturelles, n०32, p. 20-23.

Puren, Christian (s.d.) La pédagogie de projet dans la mise en œuvre de la perspective actionnelle, disponible sur www.christianpuren.com. [Page consultée le 2 octobre 2020.]

Renaut, Alain (2009), Un humanisme de la diversité : essai sur la décolonisation des identités, Paris, Flammarion.

Schmidt, Prisca (2006), « Le théâtre comme art de l'apprentissage de la langue étrangère », Spirales, n०38, p. 93-109.

Sen, Amartya (2009), L'idée de justice, Paris, Flammarion.

Sérusclat-Natale, Maud et Yannick Marzin (2020), « Les jeux de la recherche collaborative : le cas du projet artistique », Recherches en didactique des langues et des cultures, vol. 17, $n^{\circ} 2$, disponible sur: http://journals.openedition.org/rdlc/7712. [Page consultée le 3 août 2020.]

Stora, Benjamin (2019), La culture et les migrants, rapport remis au ministre de la Culture, disponible sur https://benjaminstora.univ-paris13.fr/images/stories/actualites/STORA_Mission_ Rapport_vers_trav.pdf. [Page consultée le 2 octobre 2020.]

Veret, Thomas (2018), « Compte rendu de Pierre Escudé (dir.), "Langues et discriminations", Les cahiers de la $L C D, n^{\circ} 7$ », Lectures, disponible sur http://journals.openedition.org/lectures/32399. [Page consultée le 2 octobre 2020.] 


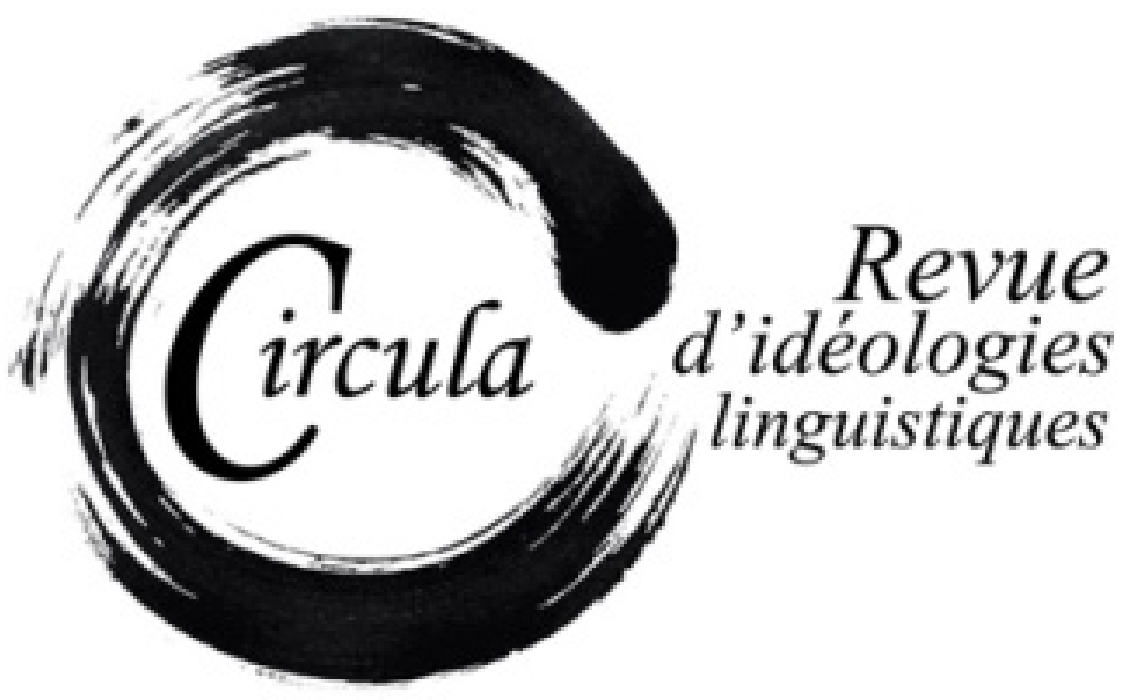

TITRE: JÓVENES DE TELEBACHILLERATO Y ALFABETISMOS DIGITALES. ENTRE EL ACCESO Y LA DESIGUALDAD

Auteur: María Guadalupe Velasco Giles, Instituto Superior de Ciencias de la Educación del

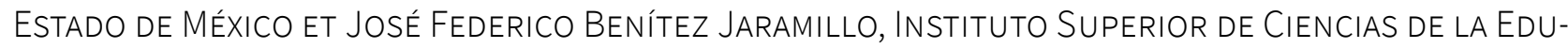
CACIÓN DEL ESTADO DE MÉXICO

Revue: Circula, NumÉro 12 : LA VULNÉRABILITÉ LINGUISTIQUE

DiRECTRICE: CLAUDIA TORRES CASTILLO

PAGES: $132-151$

ISSN: 2369-6761

URI: HTTP://HDL.HANDLE.NET/11143/18446

DOI: HTTPS://DOI.ORG/10.17118/11143/18446 


\title{
Jóvenes de Telebachillerato y alfabetismos digitales. Entre el acceso y la desigualdad
}

María Guadalupe Velasco Giles, Instituto Superior de Ciencias de la Educación del Estado de México maria.velasco@isceem.edu.mx

José Federico Benítez Jaramillo, Instituto Superior de Ciencias de la Educación del Estado de México jose.benitez@isceem.edu.mx

\begin{abstract}
Resumen: En el artículo, develamos prácticas de escritura realizadas por un grupo de jóvenes que asisten a un Telebachillerato Comunitario utilizando los dispositivos tecnológicos para comunicarse, relacionarse e interactuar a través de mensajes en los que alternan una variedad de lenguajes, símbolos, imágenes con textos. La perspectiva de alfabetismos digitales nos convoca a redefinir la cultura escrita, ya no solamente como la adquisición de la técnica del sistema de escritura, sino como actos que cuestionan las prácticas impuestas en la escuela. Las alfabetizaciones hoy implican prácticas y sentidos distintos a los que se evocan en la visión nostálgica de la escuela tradicional. La Investigación Tallerista fue una apuesta de trabajo horizontal, dialógico y colaborativo con agentes educativos. La narrativa oral y escrita, un recurso metodológico para escuchar las lecturas del mundo escolar. La categoría de interacción permitió articular aspectos como la comunicación, la relación intersubjetiva y los símbolos de carácter social.
\end{abstract}

Palabras clave: Telebachillerato; alfabetismos digitales; acceso y desigualdad

Abstract: In the article, we reveal writing practices carried out by a group of young people who attend a Community Telebachillerato using technological devices to communicate, relate and interact through messages in which a variety of languages, symbols, images and texts alternate. The perspective of digital literacy calls us to redefine written culture, no longer just as the acquisition of the technique of the writing system, but as acts that question the practices imposed in the school. Literacy today involves practices and meanings that are different from those evoked by the nostalgic vision of traditional schooling. The Workshop Research was a commitment to horizontal, dialogical and collaborative work with educational agents. Oral and written narrative, a methodological resource for listening to readings from the school world. The category of interaction made it possible to articulate aspects such as communication, inter-subjective relationships and social symbols.

Keywords: Telebachillerato; digital literacy; access and inequality 


\section{Introducción}

Los resultados aquí presentados se inscriben en la investigación colegiada del Cuerpo Académico Educación y poder. Acciones con grupos en condición de pobreza y contextos de vulnerabilidad que, desde una perspectiva sociocultural, problematiza las interacciones educativas en favor de escuelas participativas y equitativas. Epistémica y metodológicamente apuesta por la horizontalidad en la construcción del conocimiento, convocando al diálogo e interacción pedagógica a través de lo que denominamos investigación tallerista con jóvenes en diversas comunidades educativas.

Desarrollamos la investigación a través de la identificación de ejes de problematización que articulan Líneas de Generación y Aplicación de Conocimientos los cuales permitieron la construcción colectiva del problema de estudio. El primer Eje problemático se denominó Ejercicio del poder; el segundo Interacciones educativas y alfabetismos emergentes; (Eje que recuperamos para la construcción del presente artículo) y, el tercero, Comunidades educativas: participación, convivencia y nuevas territorialidades.

Compartimos la experiencia de investigación desarrollada en el 2018 con jóvenes de un Telebachillerato Comunitario del municipio de Tejupilco, situados en la Región Sur del Estado de México, a través de un taller nombrado: Narrativas de mi experiencia escolar. Emociones, relaciones y aprendizaje, cuyo taller potenció el encuentro dialógico con los y las jóvenes de un grupo de tercer grado de bachillerato, además de documentar sus voces, imágenes, escrituras; pero también sus problemáticas, gustos, tensiones y posibilidades de acceso a las tecnologías.

El objetivo que nos proponemos es develar otras expresiones lingüísticas utilizadas por jóvenes desde lo que hemos llamado alfabetismos digitales; además de conocer cuáles son las prácticas que tensan y ponen en conflicto su estar en la escuela, espacio que niega la posibilidad de coexistir junto con ellos. Es decir, los y las jóvenes cristalizan prácticas que refieren otros sentidos, otras formas de comunicación oral y escrita en el marco de sus necesidades, intereses y deseos que son soslayadas por la institución y por los tomadores de decisiones.

Entendemos los alfabetismos digitales no so lo como la lectura y la escritura con el uso de las tecnologías, sino además como "el dominio de los códigos, lenguajes y formas expresivas audiovisuales, hipertextuales, multimedia e interactiva” (Area, Gros y Marzal, 2014: 10). En la actualidad, los jóvenes suelen mezclar palabras con imágenes estáticas o en movimiento agregando música y sonido; no son solo consumidores pasivos sino también productores de sus propios textos, videos y ediciones, "para muchos jóvenes, escribir textos es sólo una forma de expresar ideas. Poco a poco están descubriendo que escribir con una mezcla de imágenes digitales, sonido y video es mucho más interesante y atractivo" (Knobel y Lankshear, 2013: 193). En nuestra visión se trata no sólo de mezclas de actividades, sino de alfabetismos en los que los diálogos tecnológicos con las letras virtuales, impresas, visuales se encuentran y desencuentran en la vida cotidiana de las personas. 
El escrito se estructura en cinco apartados: en primer término, se describe la situación problemática; un segundo momento presenta los planteamientos metodológicos y teóricos que dieron sustento a la investigación; el tercero alude a los alfabetismos digitales. Acceso y desigualdad; el siguiente describe el Telebachillerato Comunitario (en adelante TBC) como un espacio escolar en el Sistema Educativo Mexicano; enseguida recuperamos la experiencia de trabajo con los y las jóvenes estudiantes a través de sus alfabetismos digitales desde la investigación tallerista; para, finalmente cerrar con una serie de reflexiones que posibilitan seguir pensando en la emergencia de otros códigos de comunicación entre los jóvenes.

\section{Problematización}

El eje Interacciones educativas alfabetismos emergentes, implicó el reconocimiento de maneras distintas en el uso social del lenguaje, debido a que éstos se inscriben en un contexto de constantes transformaciones sociales y culturales, en el que el uso de las tecnologías como artefactos culturales da origen a otras formas de comunicación utilizando lenguajes que distan de los convencionales. El tema de la emergencia genera la posibilidad de reconocer estas prácticas situadas (Haraway, 1995), de tal suerte que proponemos el supuesto de que los alfabetismos digitales tienen un constitutivo central en la emergencia del sujeto educativo, social y colectivo en las formas en que se comunican a través de lenguajes virtuales. La emergencia hace también referencia no a un salir de la nada, sino a la visibilización situada, es una aportación no para dar la voz, sino para comprender en diálogo, desde una diversidad de lógicas que tensan, los procesos, prácticas, interacciones y narrativas que configuran a la juventud.

En este sentido, consideramos que surgen otros lenguajes a la hora de usar la palabra que permiten cuestionar no solamente las prácticas de comunicación que se pretende entablar, sino también las formas en cómo se comunican, con quiénes, para qué fines y con qué recursos (Giddens, 1998). De ahí que nos preguntamos ¿Cómo y con quiénes se comunican los jóvenes a través de alfabetismos digitales?, ¿Cuáles son los alfabetismos digitales presentes en su vida cotidiana?, ¿Con qué intención/es se comunican?, ¿Con qué sentidos?

Lo anterior, nos permite plantearnos que los cambios sociales y culturales que emergen desde la aparición de las tecnologías digitales, han derivado en transformaciones en las formas de comunicación, interacción y relación entre los sujetos; por lo que se requiere del análisis de estos fenómenos desde un enfoque sociocultural que recupere los diferentes niveles que los constituyen: social, cultural y comunicacional (García Canclini, 2004), es decir, recuperar las prácticas de comunicación situadas de las y los jóvenes. 
De ahí que, ante las transformaciones en el escenario sociocultural, político y económico no solo global sino también regional y local, algunos sujetos quedan excluidos debido a su condición geográfica, económica, de género y generacional, derivando en desigualdad, diferencia y desconexión (García Canclini, 2004). Así, hemos observado que varios de las y los jóvenes están desconectados no únicamente en términos del acceso a una red de comunicación sino además se quedan al margen de los beneficios que vulneran sus derechos. Hablamos del derecho en un sentido amplio, no únicamente como la posibilidad de acceso a los bienes y servicios más necesarios: la salud, la alimentación y la educación; sino, el derecho a un trabajo, a una identidad, a la participación, a un ambiente sano, a los medios digitales, ámbitos en los que lo educativo juega un papel significativo. Así, algunos sectores de la población si bien tienen acceso al sistema escolar, no tienen el poder adquisitivo para la compra de un dispositivo tecnológico, a sus programas y aplicaciones. Junto con el acceso a una computadora, resulta clave contar con acceso a internet, aunque este se ve limitado particularmente por su alto costo, sobre todo el internet de banda ancha (Sunkel, Trucco y Espejo, 2013).

En el período 2010 y 2015 se produjo un importante aumento en este indicador, con mayor fuerza en las áreas rurales, que mostraron un crecimiento del 414\%, frente a un 133\% en áreas urbanas. A pesar de este fuerte incremento en el área rural, su rezago con respecto de las áreas urbanas se mantuvo cercano a los 20 puntos porcentuales (Comisión Económica para América Latina y el Caribe, 2019: 48).

En el caso de la región latinoamericana, para los jóvenes, el acceso a las Tecnologías de la Información y de la Comunicación (TICS) es clave, tanto en los ámbitos de educación y empleo, como de conectividad en un mundo globalizado. No obstante, las diferencias socioeconómicas y geográficas dificultan su aprovechamiento.

Llama la atención la alta heterogeneidad entre países, el acceso a una computadora es inferior al $10 \%$ en áreas geográficas rurales y urbanas. Asimismo, el acceso a computadora en los hogares donde residen los jóvenes es mayor en áreas urbanas que en áreas rurales (Comisión Económica para América Latina y el Caribe, 2019: 47).

Exponemos la idea de que la juventud es contextualizada por el momento histórico en que "los jóvenes emergen como agentes sociales y, al hacerlo, plantean el necesario ejercicio de sus derechos sociales como miembros de un espacio" (Reguillo, 2000: 22), en este caso, los espacios educativos, sociales, culturales y comunitarios.

De acuerdo con datos del Centro Latinoamericano y Caribeño de Demografía aproximadamente 107 millones de personas en la región latinoamericana son jóvenes, lo que equivale al 17\% del total de la población. De estos, aproximadamente el 80\% vive en áreas urbanas (86 millones) y el $20 \%$ en áreas rurales (21 millones), diferencia que debería aumentar en los próximos años a favor de las áreas urbanas, debido en gran parte a los procesos migratorios de este grupo etario (Centro Latinoamericano y Caribeño de Demografía, 2017) 
El reconocimiento a la diversidad de prácticas de lectura y escritura de jóvenes dentro y fuera de la escuela permite sostener que la experiencia juega un papel crucial en sus procesos formativos como lectores y escritores y que está formada por un proceso complejo que involucra transformaciones dinámicas no sólo en cuanto al hacer, sino con relación en los conceptos de lectura, escritura y de sí mismos, a la intervención de los otros, a los usos que de ellos hacen y a la objetivación de estas perspectivas en la producción de textos escritos a través de los códigos escolares, a usos de tecnologías de la información y la comunicación, y las creaciones diversas que realizan con ellas.

En el contexto que habitan los y las jóvenes del TBC en el municipio de Tejupilco, ubicado en la Región Sur del Estado de México, se despliegan prácticas que visibilizan elementos de la cultura escrita; algunas de ellas no son nuevas y otras reformulan las posibilidades de encuentros en el marco de la escuela. Así, ante ciertas condiciones de desigualdad escolar, los jóvenes encuentran en los dispositivos digitales, principalmente, el teléfono celular, espacios para sus escrituras y lecturas que permiten la comunicación, la interacción y el acceso a la información que estos medios ofrecen a través de Internet.

En el taller desarrollado con los jóvenes señalaban haber aceptado ingresar el TBC debido a la gratuidad efectiva y la dinámica académica; evidencian que "aparece no sólo como una oferta que acerca la educación a aquellos lugares que no tienen otra opción, sino como la oferta que atiende a los que ya no pueden ser absorbidos por otros espacios" (Weiss, 2017: 13), aquellos que rebasan la edad establecida oficialmente para cursar la educación media superior, que han tenido trayectorias escolares "irregulares" y no cuentan con los créditos académicos para ser asignados en las escuelas céntricas, como expresaban los jóvenes. Cabe señalar que los jóvenes cuestionan las ideas de "tele" y "comunitario", con relación a lo primero advierten que no hacen uso de la televisión por ser propiedad de la telesecundaria -que tampoco hace uso de ella- que únicamente les prestan las instalaciones del edificio escolar para las clases y no los televisores o computadoras. Asimismo, cuestionan lo comunitario debido a que dicha categoría pierde sentido desde el momento en que no existe un proyecto que vincule realmente a la escuela con el espacio social.

En suma, si bien es cierto que muchas de las políticas sociales en México han soslayado los ámbitos rurales y urbano marginales; vemos que hoy día se está volteando la mirada hacia estos espacios, un caso particular es la aparición de los Telebachilleratos que surgen para comunidades consideradas dentro estos criterios. Estos espacios educativos juegan "en el imaginario juvenil un papel fundamental para considerarlo el factor más importante para conseguir un trabajo, más allá incluso de la experiencia laboral» (Instituto Mexicano de la Juventud, 2006: 16). En esta pertenencia o identidad por la escuela puede tener muchos significados y tienen que ver con los usos del lenguaje en el contexto social y cultural de una comunidad. 


\section{Planteamiento teórico y metodológico}

La metodología en la que nos apoyamos fue nombrada Investigación Tallerista, una apuesta de trabajo horizontal, plural, dialógico y colaborativo con agentes educativos (directivos, profesores y jóvenes estudiantes) que emerge de las inquietudes, problemáticas y necesidades de los propios sujetos que habitan la escuela, en la búsqueda de alternativas para la mejora educativa y la generación de conocimiento. La propuesta se adscribe a una perspectiva epistémica decolonial (De Sousa, 2010) que nos distancia de un trabajo extractivo de la investigación, para visibilizar o construir procesos político-pedagógicos de co-producción de conocimiento que potencien interacciones participativas y equitativas desde adentro y con las comunidades educativas, a partir del reconocimiento de sujetos epistémicos y de acción en la que colaborativamente con las y los jóvenes desarrollamos la investigación, haciendo hincapié de no construir conocimiento sobre los jóvenes, sino con los y las jóvenes.

La narrativa de y con los jóvenes estudiantes permitió una articulación compleja de la biografía/ acción/memoria susceptible de ser reflexionada a través de diversas mediaciones simbólico-narrativas (saberes, experiencias, lenguajes, normatividad, identidades, ideologías, valores, entre otros) en espacialidades y temporalidades diversas en las que se mueven los jóvenes.

El taller "Narrativas de mi experiencia escolar. Emociones, relaciones y aprendizajes" (2018) se desarrolló en las últimas dos horas de clase que reciben los jóvenes, donde en promedio asisten a clases que se desarrollan por las tardes un grupo de 20 jóvenes (13 mujeres y 7 varones cuyas edades oscilaban entre 17 y 21 años) con trayectorias sociales y escolares diversas; por ejemplo: algunos son padres o madres de familia, vivían en pareja o asistían con sus hijos a clases. Asimismo, varios llegaban al plantel expulsados de otras escuelas o se encontraban en condición de rezago educativo además de que la mayoría trabajaba por las mañanas.

Teóricamente ubicamos a la interacción como categoría que articula aspectos que tienen que ver con la comunicación, la relación intersubjetiva y los símbolos de carácter social. En este sentido, del Interaccionismo Simbólico retomamos las interacciones como construcción social y el lugar de la significación en la interpretación del mundo (Blumer y Mead, citados en Woods, 1998); De Bárcena y Mèlich (2014) se usa la idea de educación en su sentido ético y estético que supone que en la relación hay una presencia de otro frente al cual existe una responsabilidad. Finalmente, de Berlanga (2018) consideramos a la interacción como el encuentro intersubjetivo que se relaciona en encuentros y desencuentros con los otros desde la alteridad.

Así, el Interaccionismo Simbólico como lo plantea Blumer (1982), permitió considerar que, a partir de las interacciones, los jóvenes entablan la comunicación y la relación a través del lenguaje escrito alternando símbolos e imágenes en diversas superficies materiales y simbólicas. En razón de lo anterior, nos acercamos a la categoría de interacción más allá de una relación de dos personas, sino como una inter-acción, lo que supone relación con compromiso ético, habitada por nuestros proce- 
sos identitarios en co-presencia y en escenarios sociales que dan contenido al sentido común de la vida social, a su fragilidad, que implica al otro, que no está dada y que está siempre en posibilidad de configuración bidireccional, tanto de nuestros mundos simbólicos como de los contextos mismos.

La construcción de la mirada teórica hizo posible comprender las interacciones que los jóvenes del TBC entablan a través de los alfabetismos digitales para hacer lecturas de mundo a través de lenguajes y símbolos, en los que ponen de manifiesto sus preocupaciones e intereses. Así, nuestras interpretaciones van en el sentido de develar las diferentes interacciones que los jóvenes establecen con los lenguajes virtuales, con el entorno social que lo circunda y con los Otros con los que se comunica a través de mensajes orales y de texto, como son: comentarios, publicaciones, videos, memes, que se convierten en expresiones juveniles que expresan y comunican sus maneras de mirar y percibir sus mundos.

En torno a la comunicación suscribimos la tesis de que nos lleva a reconocer "nuevas expresiones políticas que inventan otros signos y desbordan las retículas de las grandes instituciones" (Reguillo, 2005: 11). No se trata pues de una perspectiva lingüística, en la que hay un emisor y un receptor en el acto de comunicarse e interactuar con el Otro; más bien, pretendemos recuperar una perspectiva sociocultural que reconoce la presencia del diálogo, la conversación y la interacción entre los sujetos que se comunican con ciertos propósitos e intenciones, lo que lleva a reconocer formas de comunicación dinámicas, cambiantes, pero que también retratan la desigualdad con relación al acceso a las tecnologías y a los múltiples lenguajes.

\section{Alfabetismos digitales. Acceso y desigualdad}

Consideramos relevante comprender las formas de vivir la juventud y el papel que juega la lengua escrita, por lo que la comprensión de sus códigos lingüísticos y de los motivos de la formación de una jerga particular puede dar un entendimiento de la naturaleza de la cultura juvenil, lo que a su vez "puede representar un factor, entre otros, en la programación de ayudas y en la superación de las barreras de comunicación”. (Zimmermann, 1996: 478), en este sentido, en la creación de variedades lingüísticas es necesario dar cuenta del lenguaje juvenil, ya que éste representa una de las variedades dentro de un sistema lingüístico en el que históricamente se construyen y re-producen cambios. Por tanto, damos cuenta de como el lenguaje juvenil se diferencia de este lenguaje estándar; en el uso de lenguajes digitales, ya que "los jóvenes luchan contra los tres aspectos: la norma escolar, el estilo culto y la cultura de los adultos" (Zimmermann, 1996: 483), es decir, sus prácticas digitales desbordan el alcance del pensamiento escolary adultocéntrico. Por ende, los "jóvenes" representan, sin duda, el grupo que ha creado la forma idiomática que más llama la atención. Para sí mismos "un símbolo de identidad sociolingüística" (Zimmermann, 1996: 484). 
Aludimos al acceso, como la posibilidad que tienen los jóvenes "para participar en eventos de lengua escrita en las cuales el sujeto se posiciona vis-a-vis con otros lectores y escritores y, a las oportunidades y modalidades que tienen para escribir" (Kalman, 2004: 8), en el caso expuesto, al hacer uso de los dispositivos tecnológicos como medios para plasmar sus lecturas y escrituras de mundo. De ahí que, no solo basta con tener un dispositivo sino además contar con el servicio de Internet que posibilite el acceso a las diferentes opciones que éste ofrece: sitios web, plataformas, aplicaciones, entre otras. Espacios virtuales que ofrecen diferentes opciones para comunicarse e informarse. Además de ofrecer una vasta variedad de textos que los jóvenes pueden leer.

Los jóvenes se mueven en infinidad de símbolos en los que construyen sus mundos y le dan sentido (Mélich, 1998), es decir, habitan espacios en los que vehiculizan y dan uso a los conocimientos que adquieren en la escuela, pero también hacen uso de otros códigos lingüísticos y símbolos, que emergen en el contexto sociocultural en el que se encuentran inmersos; ya que la cultura juvenil crea un espacio comunicativo (Zimmermann, 1996).

En este sentido, la escritura de los jóvenes no es "necesariamente lineal ni deductiva, tal como lo implica la inscripción de un texto sobre una página, sino que puede ser abierta, expandida y relacional gracias a la multiplicación de los vínculos hipertextuales" (Chartier, 2010: 36), en el que el texto electrónico reintroduce en la escritura un lenguaje simbólico y una lectura multimodal. De ahí que,

En el mundo contemporáneo en relación con la textualidad electrónica se esboza un nuevo idioma formal, inmediatamente descifrable por cada uno. Es el caso de la invención de los símbolos, los "emoticons", como se dice en inglés, que utilizan de una manera pictográfica algunos caracteres del teclado (paréntesis, comas, punto y comas, dos puntos) para indicar el significado de las palabras: alegría :-) tristeza :-( ironía ;-) ira :-) @ [...] llustran la búsqueda de un lenguaje no verbal que, por esta misma razón, pueda permitir la comunicación universal del registro del discurso (Chartier, 2010: 200-201).

Así, los y las jóvenes construyen sus propias simbologías para comunicarse haciendo uso de su imaginación, articulan diversos modos de comunicación en una red interactiva, en hipertextos que integran como parte de su historicidad en las modalidades escrita, oral y audiovisual (texto, imágenes y sonido), interactuando desde múltiples puntos, en un tiempo elegido (real o demorado) a lo largo de una red global, con acceso abierto y asequible, cambiando radicalmente el carácter de la comunicación humana (Castells, 2009). Por tanto, las tecnologías digitales de la información y la comunicación conforman entornos semióticos novedosos que son necesarios mirar desde los espacios escolares y sociales.

No se trata sólo de analizar a los sujetos en un espacio geográfico concreto, sino también analizar las relaciones lingüísticas que establecen con estos espacios, con las diversas formas de posicionarse de ellos y con el sentido de pertenencia y diferenciación que en muchas de las ocasiones los excluyen, ya que: 
La relativa unificación globalizada de los mercados no se siente perturbada por la existencia de diferentes y desiguales: una prueba es el debilitamiento de estos términos y su reemplazo por lo de inclusión o exclusión [...] los incluidos son quienes están conectados y sus otros son los excluidos [...] ahora el mundo se presenta dividido entre quienes tienen [...], acceso a la información y el dinero, y, por otro lado, los que carecen de tales conexiones (García Canclini, 2004: 73-74).

La construcción de la mirada de alfabetismos emergentes implica el reconocimiento de maneras distintas en el uso social del lenguaje, reconociendo las otras formas de comunicación y no excluirlas; debido a que emergen otras performatividades que se hacen en ciertas superficies materiales y simbólicas de escritura; que cuestionan no exclusivamente las formas de comunicación que se pretende entablar, sino con quiénes, para qué fines y con qué recursos; éstas dan cuenta de la agencialidad de los jóvenes para expresar su decir a través de la palabra escrita, ya no sólo impresa en la superficie de un papel o una pizarra sino a través de lenguajes que representan "nuevas formas de pensar el mundo y responderle" (Lankshear y Knobel, 2010: 43), dando origen a otras textualidades no reconocidas por la sociedad y las instituciones escolares, quienes parecen mantenerse estáticas frente a las transformaciones sociales y culturales que acontecen al exterior.

Derivado de lo anterior, surge una divergencia generacional y una incompatibilidad entre lo que la escuela ofrece a los jóvenes en torno a las prácticas de lectura y escritura y lo que ellos demandan y necesitan de acuerdo con intereses, inquietudes y necesidades. Por ello se afirma al respecto que:

La escuela está en crisis [...], esa institución, pensada como una tecnología —es decir, como un dispositivo, como una herramienta o un complejo artefacto destinado a producir algo-, es incompatible con los cuerpos y las subjetividades de los chicos de hoy en día (Sibilia, 2012: 135).

En tal sentido, el cuerpo y la subjetividad de los jóvenes se encuentran en diputa con la escuela, quien insiste en vestir y controlar los cuerpos, en uniformar los pensamientos, las acciones y el hacer de los jóvenes.

Al preguntar qué significa hoy ser joven, encontramos que la sociedad que se responde que su futuro es dudoso o que no sabe cómo construirlo está contestando a los jóvenes no sólo que hay poco lugar para ellos. Se está respondiendo a sí misma que tiene baja capacidad, por decir así, de rejuvenecerse, de escuchar a los que podrían cambiarla (García Canclini, 2004: 168).

Hoy día los usos diversos que innovan los jóvenes de lo virtual y por primera vez en la historia de la humanidad, vemos una nueva generación que está capacitada para utilizar la tecnología mejor que sus padres. En términos de Mead (2006), estamos frente a una cultura "prefigurativa", en la que son los jóvenes quienes enseñan a sus padres los usos que se les dan a diversos artefactos tecnológicos, 
como es el caso del celular, la computadora, entre otros, sin embargo, se considera que se está ocasionando una ruptura con la escuela, espacio donde no existe la apertura a otras expresiones que los jóvenes hacen visibles a través de las diversas prácticas sociales de alfabetismos emergentes. Por consiguiente, es importante comprender el mundo simbólico del tiempo y espacio de los jóvenes y su vínculo pedagógico con las redes electrónicas digitales y con las formas de escribir y de leer la realidad.

Por tanto, reconocemos que la presencia y el uso de las tecnologías digitales ha dado origen a otras formas de relación, comunicación e interacción entre las nuevas generaciones, y entre éstas con la letra escrita, quienes se encuentran ante una diversidad de lenguajes virtuales: textos, imágenes y sonidos frente a la superficie plana de una pantalla luminosa que, de alguna forma, rompen o adelgazan el antiguo lazo anudado entre los textos y los objetos, entre los discursos y su materialidad. "La revolución digital de nuestro presente modifica todo a la vez, los soportes de la escritura, la técnica de su reproducción y diseminación, y las maneras de leer" (Chartier, 2010: 34), ante ello, cabría preguntarnos cómo se está leyendo, qué y para qué se lee, qué posibilidades ofrece el TBC para potenciar los alfabetismos emergentes y cómo se da acceso o no a estos nuevos alfabetismos.

\section{El Telebachillerato Comunitario en el Sistema Educativo Mexica- no}

Ante lo aludido en párrafos anteriores, es importante aclarar cómo, por qué y para qué surgen los TBC, así encontramos que ante la consigna de ampliar la cobertura en el nivel medio superior responde a las modificaciones realizadas en el marco legal, particularmente en la Constitución Política de los Estados Unidos Mexicanos en la que se estableció la obligatoriedad de la Educación Media Superior en el 2012, servicio educativo destinado a la población de entre 15 y 17 años. Con la promesa de la igualdad de acceso, equidad, calidad e inclusión, surgen además una serie de documentos oficiales en los que se plantea una educación con tales características.

En tal sentido, pensar la educación como un derecho que los sujetos jóvenes situados en contextos rurales y urbanos marginados vulneran su condición, porque además de la carencia material enfrentan también la desigualdad académica; desde el perfil profesional de quienes atienden estos espacios educativos -en su mayoría con una formación técnica-; los tiempos, horarios y contenidos; las oportunidades de acceso al nivel superior, el asumir el estigma social que los discrimina "por ser los alumnos que otras escuelas no quieren", entre otras condiciones que los colocan ante una situación de desigualdad con respecto a aquellos jóvenes que asisten a un bachillerato general.

Las desigualdades de los estudiantes no se distribuyen aleatoriamente en el sistema educativo una vez que han sido "incluidos" diversos aspectos confluyen para que, a través de un proceso de autoasignación, los alumnos con mayores desventajas atiendan a un tipo de escuela y los alumnos con mayores ventajas otro (Saraví, 2015: 67). 
El origen de los Telebachillerato Comunitarios (TBC) en México se atribuye a la carencia de instituciones de educación media superior en localidades pequeñas donde no resulta factible establecer un plantel en cada una de ellas. Inicialmente este servicio educativo estuvo pensado para comunidades con características de ruralidad; sin embargo, hoy día se pueden encontrar también en zonas urbano marginales de algunas ciudades. Estas escuelas deben establecerse en comunidades con una población menor a 2500 habitantes, que no cuenten con oferta de Educación Media Superior (EMS) en por lo menos $5 \mathrm{~km}$ a la redonda y que posean instalaciones que puedan facilitar para su funcionamiento, por ello se consideraron a las telesecundarias como opciones ideales (Secretaria de Educación Pública, 2014).

Esta propuesta educativa surge en agosto de 2013, en una etapa de pilotaje con 253 centros; en 2014 se incrementó a 1497; en agosto de 2015 se abrieron 1168 teniendo un total de 2918 centros que atienden una matrícula de 104 mil estudiantes y donde participan 8859 docentes. Las cifras más recientes de noviembre de 2018 refieren la existencia de 3309 planteles en el país que atienden 139,193 estudiantes. Este subsistema es el que representa una mayor expansión en los últimos años debido a los bajos costos que implica su instauración, pues las clases se desarrollan, en su mayoría, en las instalaciones de escuelas telesecundarias cuya infraestructura y mobiliario es utilizado por los profesores y estudiantes de esta modalidad; los turnos son vespertinos por lo que, muchos de los profesores que los atienden trabajan por la mañana en otros niveles educativos.

La planta docente es reducida (únicamente tres docentes) y alguno de ellos también cumple las funciones de Director. Cada maestro se encarga de impartir una serie de materias divididas en tres áreas disciplinares: Matemáticas y Ciencias Experimentales; Ciencias Sociales y Humanidades; y Comunicación. La modalidad educativa es presencial, los alumnos realizarán 80\% de actividades de aprendizaje bajo la mediación del profesor y el 20\% bajo el esquema de estudio independiente.

Estas condiciones de desigualdad y exclusión se hacen presentes en los jóvenes que asisten al Telebachillerato de "El Molino", en una colonia urbano marginal del municipio de Tejupilco, en el Estado de México. Esta colonia se ha caracterizado por ser habitada por familias que han emigrado de los estados de Michoacán, Guerrero y Morelos, principalmente, lo que la convierte en un mosaico sociocultural. Sin embargo, las situaciones por las que llegan a este espacio es porque están huyendo de los grupos delincuenciales o para mejorar sus condiciones económicas y sociales, lo que acentúa la desigualdad y la exclusión; como lo puntualiza Saraví (2015), no tienen la oportunidad de elegir otra escuela, sino que la escuela los elige a ellos por su condición de ser joven vulnerado y vulnerable. Empero, esta diversidad cultural que se hace visible en las aulas y en el espacio escolar, enriquece la comunicación y el lenguaje mediante sus expresiones emergentes. De ahí que consideremos que el lenguaje "es un medio por el cual el hombre puede comunicarse con el otro, es un juego de codificación y decodificación de signos y símbolos que posibilitan la comprensión y transmisión de información” (Espejel, 2014: 79). 


\section{Los jóvenes del Telebachillerato y sus alfabetismos digitales}

Como hemos venido señalando, las formas de comunicación que comparten los jóvenes son diversas, ya que ellos, pese a su procedencia geográfica y social, convierten el espacio escolar en un encuentro dialógico diversificado culturalmente; lo que les coloca ante la necesidad de apropiarse de los códigos lingüísticos que no le son propios y que los coloca ante una condición de desigualdad lingüística. En este sentido, la palabra escrita juega un papel importante en la comunicación, pero también en el logro de los vínculos sociales, afectivos y pedagógicos.

Algunos ejemplos de ello son las siguientes expresiones TQM que es la abreviatura de las palabras "te quiero mucho"; NTP para expresar "no te preocupes"; ADV (Asco De Vida), pero también tiene su versión en inglés FML (Fuck My Life) y sirve para expresar que algo no ha salido como esperaban, sino bastante peor. Otras abreviaturas derivadas del idioma inglés son: ILY para abreviar las palabras "I love you"; BFF Best Friends Forever "mejores amigos para siempre"; además de algunas letras y números como los siguientes: "X2" que para los jóvenes significa compartir el punto de vista de otra persona que expresa una opinión en redes sociales; "+1" es una de las abreviaturas más famosas y se utiliza para expresar que algo que les gusta mucho y si quieren mostrar más énfasis en ello, pueden añadirle ceros "+10" a los cuales suelen agregar emoticones para expresar emociones o estados de ánimo, como por ejemplo: carita feliz, llanto, enojo, asombro, entre otras.

Esta serie de palabras, signos y emoticones son significativas en la comunicación de los jóvenes; sin embargo, muchos de los códigos no son siempre comprendidos por algunos de los jóvenes, quedando siempre resquicios en la comunicación. Los jóvenes usan estas expresiones porque les permite sentirse diferenciados, pertenecer a un grupo y fortalecer la empatía entre iguales; pese a que no son aceptadas por la mirada adultocéntrica, ellos potencian el establecimiento de códigos que lo diferencien de otras generaciones creando su propio mundo. De ahí que sea importante conocer el significado de estas expresiones para poder entender las relaciones en las que convergen.

Durante una de las sesiones del taller que se desarrolló con los jóvenes estudiantes del Telebachillerato Comunitario, se propuso trabajar el Eje: "Escrituras en red", las preguntas que posibilitaron el diálogo fueron: ¿Con quiénes me comunico e interactúo en las redes sociales?, ¿Qué escribo en la Internet?, ¿Qué leo en Internet?, ¿A qué páginas acceso con mayor frecuencia?, ¿Utilizo la Internet para investigar algún tema o tarea escolar?

De los 20 jóvenes participantes, solo 18 enviaron sus respuestas al número de WhatsApp que previamente les compartimos. En el diálogo que surgió a partir de sus respuestas, los jóvenes comentaron que la red social de mayor uso es Facebook 50\% (9 estudiantes), seguida de WhatsApp 22.2\% (4), Instagram 16.6\% (3), y Twitter 11.1\% (2), espacios virtuales en los que realizan escrituras y lecturas digitales. Cabe señalar que algunas de estas plataformas digitales son de carácter público, es decir cualquier persona puede tener acceso a sus datos, por lo que algunos jóvenes usan nombres anónimos o algún seudónimo. Mientras que en WhatsApp utilizan su número personal del celular, lo 
que le otorga un carácter más privado. Los jóvenes establecen diferencias entre una red social y una aplicación; señalan Facebook, Instagram y Twitter como redes sociales; mientras que a WhatsApp la ubican como una aplicación. Además, utilizan más de una red social, así que los datos ofrecidos responden a cuál de ellas pusieron como primera, segunda y/o tercera opción.

Entre las personas con las cuales se comunican colocan en primer término a sus amigos y/o amigas 94.4\% (17 jóvenes) y 5.5\% a la familia (1 joven). De igual manera, ponían una primera, segunda o tercera opción; por ejemplo, 2 de los jóvenes colocaron a sus parejas sentimentales como segunda opción, después de sus amigos. Así también, solo una joven comentó que usa WhatsApp para comunicarse con sus maestros.

Con respecto a las publicaciones que realizan, 9 (50\%) dijeron que "memes"; 8 (44.4\%) fotografías personales y solo un joven publica noticias (5.5\%). En su mayoría, los jóvenes utilizan las redes como diversión o recreación, para enterarse de lo que otros publican. Es en estos espacios virtuales donde las y los jóvenes realizan lecturas y escrituras digitales, 5 (27.7\%) expresan leer lo que otros publican; 4 leen noticias (22.2\%) aunque no especifican de qué tipo; 2 (11.1\%) leen memes y 7 (38.8\%) no escriben ni leen en redes. Entre sus segundas opciones, una joven comentó que escribe sus estados de ánimo o frases motivacionales que puedan ayudar a quienes las leen; otra dijo interesarse por leer recetas de cocina.

Los 18 jóvenes (100\%) que enviaron sus respuestas a través de WhatsApp aceptaron hacer consultas en la Internet para sus tareas escolares. Las plataformas y páginas de mayor acceso son Google Académico 13 (72.2\%); Wikipedia 2 (22.2\%); Redalyc 2 (11.1 \%) y Webassing 1 (5.5 \%); 11 (61.1\%) jóvenes han leído algún libro en Internet y 7 (38.8\%) no han realizado lectura de libros electrónicos. En cuanto a sus preferencias literarias 5 (27.7) muestran agrado por los textos de ficción; otros 4 (22.2\%) por las novelas y 2 (11.1\%) por textos científicos.

Los datos anteriores develan que los jóvenes realizan sus lecturas y escrituras digitales de acuerdo con sus intereses, necesidades, emociones y motivaciones. Los propósitos son diversos y van desde la diversión y esparcimiento hasta enterarse de noticias, comentar las publicaciones de otras personas o manifestar su opinión sobre los temas que ahí se exponen.

Otra de las actividades de escritura que se les propuso a los jóvenes participantes en el taller fue elaborar un "meme" haciendo uso de sus celulares; bajo las preguntas: ¿Qué me gusta de la escuela?, ¿Qué me disgusta? Si pudiera, ¿Qué cambiaría de la escuela?

Previo a que dieran inicio con la actividad, les preguntamos si sabían que era un meme. Después de escuchar algunas opiniones les compartimos la siguiente información: 
El meme más allá de imágenes cómicas y del señalamiento de algunas figuras reconocidas (como artistas, políticos, deportistas, etc.) se trata de enunciaciones que responden a una amplia variedad de satisfactores [...] puede tener muy diversas formas de expresión cultural. Puede ser desde una frase (los refranes populares serían un buen ejemplo), una imagen (la Mona Lisa reproducida en litografías, camisetas, entre otros), o bien, presentarse a partir de diversas formas simbólicas disponibles en internet, como videos virales o imágenes que son reproducidas a través de las redes sociales (Pérez Salazar, 2014: 113-115).

Cabe señalar que, en términos pedagógicos, la elaboración de un meme implica el desarrollo de habilidades como la imaginación, la creatividad, el uso y manejo de diversos recursos materiales y tecnológicos de los cuales los jóvenes echan mano para realizar sus creaciones. Así mismo, requiere el manejo de las tecnologías como artefactos culturales que demandan otras formas de leer y escribir el mundo. De esta manera el joven no es únicamente consumidor pasivo de las escrituras de otros sino también es productor de sentidos y significados a través de lo que escribe y expresa por medio del celular. De ahí que, las escrituras digitales muestran a los jóvenes de la escuela como sujetos que aprenden el manejo técnico de un aparato, pero también aprenden otras maneras para comunicarse, interactuar e informarse mediante la letra escrita. Para desarrollar la actividad se formaron equipos de cuatro integrantes, quienes tomaron acuerdos con respecto al tema del que hablarían en el meme. Se puntualizó el tiempo que dispondrían para desarrollar la actividad. Algunos jóvenes salieron del espacio (el laboratorio) que se nos había facilitado para desarrollar las sesiones del taller y hacer un recorrido, tomar fotos o simplemente para trabajar afuera. A su regreso compartimos nuestro número de WhatsApp para que nos enviaran sus elaboraciones, algunos intercalaron imágenes, fotografías y textos; otros, imágenes bajadas de la red con textos escritos por ellos mismos. Los memes se proyectaron utilizando un cañón para que pudieran ser observados por todo el grupo y los integrantes de los equipos explicaran su contenido. Enseguida mostramos las elaboraciones escritas de algunos equipos.

El primer equipo muestra un "meme" con la imagen de "Patricio" arrastrando a "Bob Esponja" (personajes de una caricatura infantil); la imagen es descargada de Internet, tiene como encabezado la frase "When te hacen bullying y la orientadora solo Te engaña diciendo QYa No Pasará"; en la parte inferior de la imagen hay otra frase que dice: "No te preocupes, estarás bien"; los jóvenes agregaron el texto: "Cuando Le Decimos A Los maestros algo \& no hacen nada".

Otro equipo refiere una fotografía de la cancha de futbol rápido, en la cual los jóvenes manifiestan su inconformidad por el descuido en que se encuentra por la falta de mantenimiento en esta área de esparcimiento y deporte. La foto va acompañada de un texto escrito por ellos mismos "Una De Las Principales Areas Q necesitan Un Mejoramiento Para Todo El Alumnado \& Docentes Para Poder Realizar Distintas Actividades Físicas O Juegos Deportivos". (La transcripción de los empíricos aparece tal cual fueron escritas por los jóvenes). 
Cabe destacar en estos empíricos el uso que hacen los jóvenes de algunos términos en inglés como "when" y "bullying", el símbolo "\&" (traducido como "and" en inglés y en español "y") de uso común entre ellos; además de usar una sola letra "Q" para enunciar la palabra "que" de manera abreviada. De ahí que, para entender el contexto en el que se mueven los jóvenes resulta fundamental entender la estructuración de los diferentes espacios y lenguajes en los que se mueven.

En este sentido, la creación de nuevas variedades lingüísticas es necesario dar cuenta del lenguaje juvenil, ya que éste representa una de las variedades dentro de un sistema lingüístico en el que históricamente se construyen y re-producen cambios lingüísticos. Por tanto, damos cuenta de cómo el lenguaje juvenil se diferencia de este lenguaje estándar. Para esto "Ios jóvenes luchan contra los tres aspectos: la norma escolar, el estilo culto y la cultura de los adultos". (Zimmermann, 1996: 483).

En suma, abordar las expresiones lingüísticas de los jóvenes a través de alfabetismos digitales aporta experiencias teórico empíricas que ayudan a mirar cómo las personas se convierten en usuarios de nuevos códigos para comunicarse, relacionarse e informarse. Esta posición tiene relación estrecha con el desarrollo teórico de los estudios socioculturales sobre las prácticas sociales de la lengua escrita (Kalman, 2004) y su apropiación por parte de los jóvenes. Los estudios que se muestran en la investigación sociocultural sugieren una especie de imbricación entre la práctica y los contextos en que éstos tienen lugar. Esto es, que las acciones desarrolladas por los jóvenes en la consecución de ciertas metas se configuran en interacción con las circunstancias que los rodean. Así, el acceso a las prácticas sociales, es un proceso contextualizado; un proceso imbricado en el desarrollo mismo de las prácticas, donde la interacción entre los aprendices constituye una mediación social.

\section{Reflexiones finales}

Hablar de alfabetismos digitales de los jóvenes nos convoca a reflexionar; en primer término, con otros lenguajes que se alejan de los convencionalismos pedagógicos y sociales establecidos históricamente; así desde el pensamiento adultocéntrico es importante abrirnos para poder remirar el mundo de escrituras y lecturas de las culturas juveniles que aportan conocimientos a los debates actuales en América Latina. De ahí que los aportes de los y las jóvenes tendrían que ser constitutivos tanto de los cambios culturales y tecnológicos como de las nuevas formas de comunicación social que se instituye como parte de la propia existencia.

Narrar las experiencias educativas de las y los jóvenes viabilizó el comprender los acontecimientos situados espacial y temporalmente en el uso de sus cuerpos donde también escriben y narran con imágenes, símbolos, códigos que se tatúan en el marco de sus historias que cristalizan en amores, desamores y utopías. Son lecturas y escrituras de poder e intersubjetividades que nos conmueven a ser escuchados. 
Asimismo, las experiencias vividas en los talleres nos permitieron comprender que existen diversos modos en el que las escrituras juveniles se hacen presentes a través de múltiples formas emergentes que se contraponen con las normas de escrituras y lecturas de la escuela y en muchas ocasiones de la sociedad en general. Por tanto, los cambios que la sociedad globalizada demanda en cuanto al uso de las tecnologías, escrituras y los mismos jóvenes que a través de sus acciones solicitan, se convierte en encuentros y desencuentros de comunicación juvenil. Sin embargo, existe la necesidad de atender su subjetividad y así aperturar la mirada hacia lo plural y diverso, dejando de lado el accionar adultocéntrico y hegemónico, con el propósito de reducir la brecha de la desigualdad social, educativa y cultural, ya que la exclusión y la segmentación desigual "son las dos principales consecuencias de la reestructuración" (García Canclini, 2004: 170).

En la multimodalidad textual observamos que los jóvenes estudiantes hacen usos diversificados de las tecnologías; apropiándose de ellos como espacios de recreación, expresión de sus sentires, deseos, emociones y experiencias de lo que viven cotidianamente. Así, la escuela como institución se ve rebasada, ya que la mayoría de los docentes que laboran en estos espacios desconocen las múltiples lecturas que los jóvenes hacen de sus recursos tecnológicos. Por lo que: "asumir posturas críticas y abiertas implica trabajar sobre el papel social de la educación y, en nuestro caso, sobre el papel social de los sujetos de la educación ante el cambio tecnológico" (De Alba, 2010: 86).

Finalmente consideramos que los y las jóvenes están ansiosos de ser escuchados, de interactuar, de develar sus experiencias de vida ligadas a su cultura, sociedad, en donde configuran saberes, subjetividades y formas particulares de escribir el mundo y de decir su palabra, sin embargo, el acceso y desigualdad se sigue acrecentando en gran parte de nuestro continente, y se hace visible en los rostros de los jóvenes, en sus interacciones y expresiones lingüísticas. 


\section{Referencias bibliográficas}

Area, Manuel, Begoña Gros y Miguel Marzal (2014), Alfabetizaciones y tecnologías de la información y la comunicación, Madrid, Editorial Síntesis.

Bárcena, Fernando y Joan-Carles Mèlich (2014), La educación como acontecimiento ético. Natalidad, narración y hospitalidad, Buenos Aires, Miño y Dávila.

Berlanga, Benjamín (2018), Educar con sujeto: experiencia, don y promesa (otro modo de relación con el otro que no sea el de intervención. Notas de una charla en el Seminario "Pedagogía de la indignación, pedagogía del sujeto", seminario coordinado por la UCIRED para el equipo del Centro de Derechos Humanos Victoria Diez, León.

Blumer, Herbert (1982), Interaccionismo simbólico: perspectiva y método, Bárcelona, Hora.

Castells, Manuel (2009), Comunicación y Poder, Madrid, Alianza Editorial.

Centro Latinoamericano y Caribeño de Demografía (2017), América Latina: estimaciones y proyecciones de población total, urbana y rural, y económicamente activa 1950-2050, disponible en https://www.cepal.org/es/temas/proyecciones-demograficas/estimaciones-proyecciones-poblacion-total-urbana-rural-economicamente-activa. [Sitio consultado el 6 de junio de 2020.]

Comisión Económica para América Latina y el Caribe (CEPAL) (2019), Panorama Social de América Latina, disponible en https://repositorio.cepal.org/bitstream/handle/11362/44969/5/ S1901133_es.pdf. [Sitio consultado el 29 de mayo de 2020.]

Chartier, Roger (2005), "Lenguas y lecturas en el mundo digital”, en El presente del pasado: escritura de la historia, historia de lo escrito, México, Universidad Iberoamericana, p. 195-220.

Chartier, Roger (2010), “Aprender a leer, leer para aprender”, en José Antonio Millán (ed.), La lectura en España: informe 2008, p. 23-42.

De Alba, Alicia (2010), "Internet: reto de inclusión social”, en José Carabajal Romero y Daniel Saur Moyano (eds.), El desafío tecnológico: transformaciones y fronteras educativas, México, Juan Pablos, Seminario de Análisis de Discurso Educativo, p. 79-92.

De Sousa, Boaventura, Santos (2010), Descolonizarel saber, reinventarel poder, Montevideo, Ediciones Trilce.

Espejel, Diana (2014), "La escritura del afecto: los vínculos entre los jóvenes en la cultura digital", en Sandra Flores (ed.), Redes sociales digitales: nuevas prácticas para la construcción cultural, México, Conaculta.

García Canclini, Néstor (2004), Diferentes, desiguales y desconectados: mapas de la interculturalidad, Barcelona, Gedisa.

Giddens, Anthony (1998), La constitución de la sociedad, Buenos Aires, Amorrortu. 
Haraway, Donna (1995), Ciencia, cyborgs y mujeres: la reinvención de la naturaleza, Madrid, Cátedra.

Instituto Mexicano de la Juventud (2006), Encuesta Nacional de la Juventud 2005, disponible en https://sic.cultura.gob.mx/ficha.php?table=centrodoc\&table_id=245 [Sitio consultado el 15 de octubre de 2020.]

Kalman, Judith (2004), Saber lo que es la letra: una experiencia de lectoescritura con mujeres de Mixquic, México, Siglo XXI.

Knobel, Michele. y Colin Lankshear (2013), "Remix: la nueva escritura popular", en Ana Sacristán (ed.), Sociedad del Conocimiento, Tecnología y Educación, Madrid, Morata.

Lankshear, Colin y Michele Knobel (2010), Nuevos alfabetismos: su práctica cotidiana y el aprendizaje en el aula, Madrid, Morata.

Mead, Margaret (2006), Cultura y compromiso: estudio sobre la ruptura generacional, Bercelona, Gedisa.

Mèlich, Joan-Carles (1998), Antropología simbólica y acción educativa, México, Paidós.

Mèlich, Joan-Carles (1998), Totalitarismo y fecundidad: la filosofía frente a Auschwitz, Barcelona, Anthropos.

Pérez Salazar, Gabriel (2014), "El meme en redes sociales: prácticas culturales de replicación en línea”, en Sandra G. Flores (ed,), Redes sociales digitales: nuevas prácticas para la construcción cultural, México, Consejo Nacional para la Cultura y las Artes, p. 113-132.

Reguillo, Rossana (2000), "La condición juvenil en el México contemporáneo: biografías, incertidumbres y lugares", en Rossana Reguillo (ed.), Los jóvenes en México, México, FCE-CNCA, p. 395-429.

Reguillo, Rossana (2005), Horizontes fragmentados: comunicación, cultura, pospolítica. El (des)orden global y sus figuras, Guadalajara, ITESO.

Saraví, Gonzalo (2015), Juventudes fragmentadas: socialización, clase y cultura en la construcción de la desigualdad, México, FLACSO-CIESAS.

Secretaria de Educación Pública (2014), Documento Base para el Servicio Educativo de Telebachillerato Comunitario, México, Secretaría de Educación Pública.

Sibilia, Paula (2012), "La escuela en un mundo hiperconectado: ¿redes en vez de muros?" Revista Educación y Pedagogía, vol. 24, n 62, disponible en https://revistas.udea.edu.co/index.php/revistaeyp/article/view/14199. [Sitio consultado 25 de mayo de 2020.]

Sunkel, Guillermo, Daniela Trucco y Andrés Espejo (2013), La integración de las tecnologías digitales en las escuelas de América Latina y el Caribe: una mirada multidimensional, Santiago de Chile, CEPAL, disponible en www.cepal.org/es/publicaciones/21681-la-integracion-tecnologias-digitales-escuelas-america-latina-caribe-mirada [Sitio consultado el 26 de octubre de 2020.] 
Vommaro, Pablo (2015), Juventudes y políticas en América Latina: tendencias, conflictos y desafíos, Ciudad Autónoma de Buenos Aires, Grupo Editor Universitario.

Weiss, Eduardo (2017), "El Telebachillerato Comunitario: una innovación curricular a discusión", Revista Latinoamericana de Estudios Educativos (México), vol. XLVII, n³ 3-4, p. 7-26.

Woods, Peter (1998), La etnografía en la investigación educativa, Barcelona, Ediciones Paidós.

Zimmermann, Klaus (1996). "Lenguaje juvenil, comunicación entre jóvenes y oralidad”. En Thomas Kotschi, Wulf Oesterreicher, Klaus Zimmermann (eds.), El español hablado y la cultura oral en España e Hispanoamérica, Madrid, Vervuert Iberoamérica. 


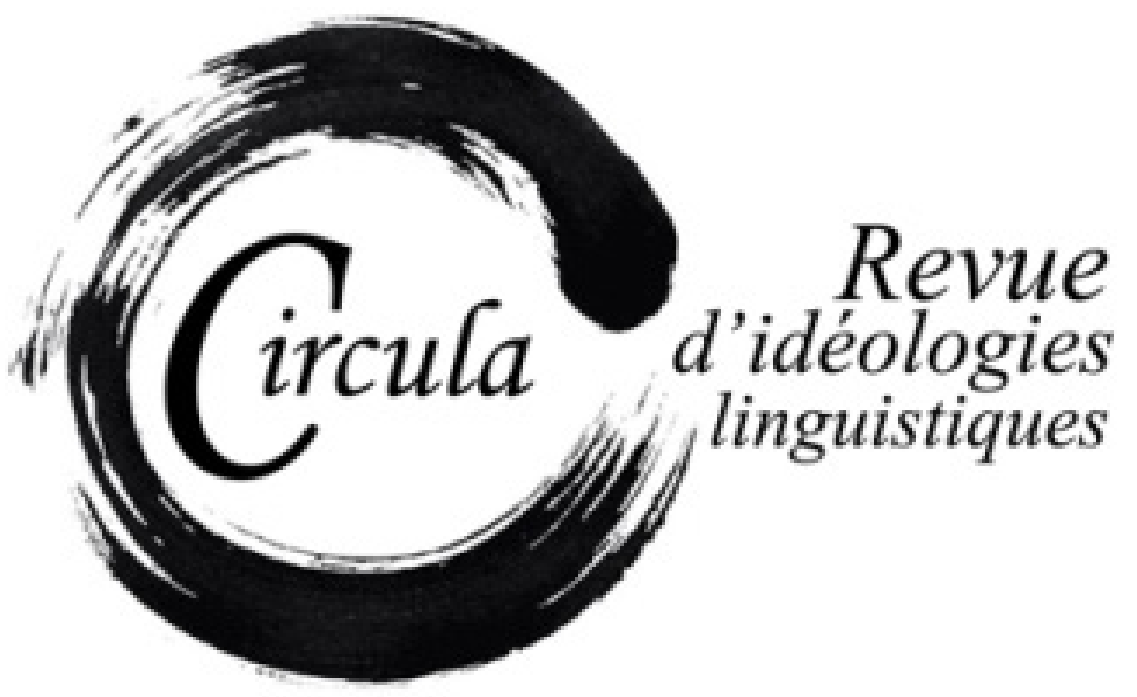

TITRE: L'ENSEIGNANT DE LANGUE ÉTRANGÈRE ET RAPPORT À LA LANGUE : COMMENT L'ENSEIGNANT INDIEN DU FLE NÉGOCIE L’AMBIVALENCE « SÉCURITÉ-INSÉCURITÉ LINGUISTIQUE »

AUTEUR: VASUMATHI BADRINATHAN, UnIVERSITÉ DE MUMBAI

Revue: CIRCULA, NumÉro 12 : LA VULNÉRABILITÉ LINGUISTIQUe

DiReCTRICE: CLAudia TORRES CASTILLO

PAGES: $152-175$

ISSN: 2369-6761

URI: HTTP://HDL.HANDLE.NET/11143/18447

DOI: HTTPS://DOI.ORG/10.17118/11143/18447 


\title{
L'enseignant de langue étrangère et rapport à la langue : comment l'enseignant indien de FLE négocie l'ambivalence « sécurité- insécurité linguistique »
}

\author{
Vasumathi Badrinathan, Université de Mumbai \\ vasumathi.badrinathan@mu.ac.in
}

Résumé : Cet article interroge le rapport avec la langue française qu'entretiennent les enseignants indiens de français langue étrangère (FLE). Seront analysées ici prioritairement les questions de la sécurité-insécurité linguistique selon les représentations des enseignants, leurs expériences affectives et les stratégies employées consciemment ou inconsciemment par les enseignants pour combler les insécurités. Une étude de type qualitative composée d'entretiens semi-dirigés avec des enseignants indiens de FLE démontre que les enseignants entretiennent un rapport complexe et ambivalent à la langue française qui contribue à leur identité d'enseignant. Ils se sentent à la fois « dedans » et « dehors », la langue enseignée étant parallèlement, source de confiance en soi et source d'impuissance, source de réconfort et d'angoisse selon les situations. Les causes de l'insécurité linguistique sont étudiées également, avec des préconisations contextualisées.

Mots-clés : rapport à la langue ; sécurité-insécurité linguistique ; affectif ; ambivalence ; enseignants de FLE; Inde

Abstract: This article looks at the relationship that Indian teachers of French share with the target foreign language. Questions relating to linguistic security and insecurity are addressed, based on teacher perceptions, their personal and emotional experiences and the strategies that they use consciously or unconsciously to overcome their insecurity. A qualitative study comprising of semi-directed interviews with Indian teachers of French reveals that teachers share an ambivalent and complex relationship with French language, which also contributes to their self-identity. They consider themselves as both "insiders" and "outsiders" at the same time, with French language posing simultaneously as a source of self-confidence and ineffectiveness, source of comfort and anxiety as per the situation. The article also studies the reasons for language insecurity and makes recommendations suited to the Indian context.

Keywords: relationship with language; linguistic security-insecurity; perceptions; ambivalence; French teachers; India 


\section{Introduction}

Comment l'enseignant se sent-il/elle devant une langue étrangère qu'il ou elle enseigne? Quel type de rapport a-t-il avec cette langue? Cette recherche étudie les représentations des enseignants sur le lien affectif qu'ils ont avec la langue française, notamment les sentiments de sécurité-anxiété et d'infériorité linguistique que ces enseignants pourraient ressentir vis-à-vis de la langue cible. Les sources de cette anxiété et les stratégies pour la combler employées par les enseignants sont également discutées. Si l'on évoque l'insécurité, les questions de « sécurité » et de confiance trouvent leur place naturellement dans cette étude. Le rapport avec la langue enseignée pour l'enseignant indien n'est pas aussi simple qu'il n'y parait. Que ressent l'enseignant sous la façade du métier? Quel rôle jouent ses représentations concernant ses compétences linguistiques en tant qu'enseignant de FLE ? Comment gère-t-il ses angoisses et ses insécurités ? Pourrait-il exister un rapport favorable de confiance avec la langue, malgré les insuffisances linguistiques? Ces questions se retrouvent au cour de cette étude, qui s'avère être parmi les premières à interroger ce lien affectif concernant les enseignants indiens de langue française. Les expériences affectives sont étudiées par le biais des représentations des enseignants, qui révèlent comment l'enseignant élabore et crée le sens qu'il confère à ses activités, à la personne qu'il est. Ces représentations ont donc une conséquence sur son identité et son activité professionnelle. Stevick (1980:4) met en valeur l'acte d'apprentissage des langues, qui porte plus sur « ce qui se passe » en classe, « dans et entre les personnes », valorisant ainsi l'affectif sur le cognitif, dont l'importance a été pleinement dévoilée par l'approche rogérienne (v. la vaste bibliographie de Rogers dont les publications de 1965 et 1967). Le Cadre européen commun de référence pour les langues (CECRL) a également pris en compte ces évolutions et mis en évidence la part de l'affectif. Évoquant l'activité de communication des apprenants, le CECRL explique qu'à part les connaissances, comptent à titre égal « les facteurs personnels liés à leur personnalité propre et caractérisées par les attitudes, les motivations, les valeurs, les croyances et les types de personnalité qui constituent leur identité » (2001 : 84). Ces facteurs comptent pour les enseignants également.

Puisque cette étude concerne l'enseignant indien de FLE, qu'on le veuille ou non, la question de l'enseignant « natif/non natif » se glisse dans le sujet de cette recherche et mérite ici un peu de discussion. L'enseignant indien de FLE est «traditionnellement » un enseignant « non natif ». Le sujet « natif/non natif » a fait l'objet des débats en recherche depuis quelques décennies. Le sujet n'a pas perdu de son éclat au vu des recherches qui témoignent de son actualité (Medgyes, 1992 ; Llurda, 2006 ; Dervin et Badrinathan, 2011 ; Dewaele, 2018 ; Houghton et Hashimoto, 2018 ; Zheng, 2018 ; Houghton et Bouchard, 2020) en remettant en cause la notion du clivage natif/non natif ${ }^{1}$. Castellotti (2011) évoque la pesanteur des « vraies langues » et les « vrais locuteurs ». Elle appelle à « dénativiser » l'enseignement des langues par une réflexion sur la compétence plurilingue et pluriculturelle. Concernant la nomenclature, Dewaele (2018) s'interroge avec raison sur l'utilité de qualifier une

1. Dans le discours anglo-saxon, la plupart des recherches sur cette question portent sur les enseignants d'anglais, d'où les acronymes fréquemment utilisés - NEST : Native English Speaking Teacher et NNEST : Non Native English Speaker Teacher. 
personne par ce qu'elle n'est pas (non natif) et par là avancer un « modèle déficient ». Il propose donc comme alternative « utilisateur LX» - LX incluant toutes langues que l'utilisateur connaît en dehors de sa L1, qu'il acquiert après l'âge de trois ans, y compris les langues étrangères, avec des degrés de maitrise variés. Ce qui nous rappelle la notion de «truncated competence » de Blommaert, Collis et Slembrouck (2005) où les chercheurs évoquent les ressources langagières possédées par le locuteur au sein des répertoires multilingues inégalement distribués. L'Inde multilingue en est un exemple, avec des ressources langagières variées et inégales utilisées par les locuteurs en fonction des situations et des milieux de communication. L'utilisateur LX de Dawaele se présente comme un terme neutre, sans valorisation ou sens d'infériorité, se rapprochant de "l'utilisateur multi compétent » de langues (Cook, 2012). Dawaele avance donc l'argument que la distinction « locuteur L1-LX» est préférable à la dichotomie «natif/non natif ». L'importance du plurilinguisme est ici sous-jacente. Ces arguments soulignent l'idée que l'identité de l'enseignant de langue étrangère, ici le FLE, est fortement façonnée par son métier ainsi que la langue avec laquelle il est intimement en contact, et non par la naissance dans une langue. Nous sommes donc soucieuse de ne pas vouloir « biologiser cette dichotomie » (Dervin et Badrinathan, 2011 : 11) et aussi de donner la juste place au social dans cette construction identitaire, ce que cette étude montre. La recherche ayant démontré à quel point cette terminologie est déficiente et faible, dans cet article nous avons consciemment évité d'utiliser le mot «non natif », qui nous parait réducteur, peu inclusif et chargé de connotations défavorables.

Qu'on le nomme «natif », " non natif » ou enseignant de langue, tout simplement, la question de la sécurité linguistique se pose surtout quand la langue étrangère enseignée est à la fois l'objet et l'outil d'enseignement et ne représente pas la langue première de l'enseignant. Aujourd'hui, grâce à l'évolution des technologies et à la mondialisation, l'accès à la langue et à ses composantes ont doublé de manière astronomique, et par là, «la proximité » avec la langue se trouve modifiée. Ce qui était auparavant « loin », tant sur le plan géographique que sur le plan culturel, est désormais à portée de main. Est-ce que cette évolution socioculturelle et technologique se révèle avoir un impact sur le rapport à la langue enseignée et réduit par conséquent l'idéal intériorisé du locuteur natif?

\section{L'insécurité linguistique}

Les premières études concernant l'insécurité linguistique ont fait leur apparition dans les années 1960, quand Labov (1966) a étudié les éléments stigmatisés et ceux de prestige dans l'anglais utilisé à New York. Les recherches ont avancé dans le domaine sociolinguistique avec des débats sur la norme, les variations langagières, la diglossie, la langue maternelle et les autres langues en question, la valorisation ainsi que l'infériorité et la supériorité des langues, provoquant l'insécurité linguistique (v. Gueunier, Genouvrier et Khomsi, 1978; Rubdy, 2001 ; Bretegnier et Ledegen, 2002 : Preston, 2013). 
Qu'en est-il de l'enseignant ou de l'apprenant de langue étrangère? Surtout, celui pour qui la langue enseignée n'est ni la langue première, ni la langue officielle du pays d'où il est originaire et où il vit? Horwitz, Horwitz et Cope (1986 : 28), dans leur étude importante sur l'anxiété des apprenants de langue étrangère, soulignent le rôle des croyances et des sentiments dans les représentations et les perceptions provenant de la singularité même de l'apprentissage des langues. Bucci et Baxter (1984) définissent l'insécurité linguistique comme la perception de soi négative d'un locuteur concernant son expression en une langue, ce qui se rapproche des propos de Meyerhoff (2006: 292) : le locuteur ressent que la langue qu'il utilise est « inférieure » ou « moins bien ». Les études démontrent que l'insécurité est souvent d'ordre linguistique - Mousavi (2007) en Angleterre, Roussi et Cherkaoui Messin (2011) sur les enseignants de français langue étrangère en Grèce, Daftari (2016), Lee, Schultz et Van Vlack (2017) sur les enseignants turcs et coréens d'anglais respectivement, Harada (2019) sur les enseignants de français au Japon à titre d'exemples.

Le français étant une langue étrangère, l'enseignant indien de FLE jouit en Inde d'une certaine sécurité liée à son statut de langue étrangère, qui lui confère un prestige, un privilège peu accordé aux enseignants de langues indiennes par exemple. Il va sans dire que la grande majorité des enseignants de français en Inde sont des Indiens. Il est donc important et indispensable de mieux connaître leur rapport à la langue, qui pourrait avoir une influence sur l'enseignement-apprentissage. II n'existe pas beaucoup de recherches qui interrogent la dimension affective et les représentations des enseignants de langue étrangère concernant leur maitrise linguistique, leurs expériences émotionnelles et l'influence sur la sécurité-insécurité linguistique. Et pourtant, il s'agit d'une dimension importante car elle influe sur les pratiques de classe et la posture enseignante (Schutz et Lee, 2014).

\section{Le français en Inde}

La situation concernant la langue française en Inde est moins compliquée que celle de l'anglais. Langue des colonisateurs, langue première pour une certaine partie de la population, langue d'instruction dans l'éducation supérieure, l'anglais est pourtant souvent remis en question, rejeté et parfois condamné. Le français est une langue étrangère en Inde et le pays ne figurant pas dans les cercles interne, externe ou grandissant de la langue française (pour emprunter au paradigme de Kachru²), le rapport à cette langue est dénué des tensions et des questions d'hégémonie linguistique qui caractérisent d'habitude l'anglais en Inde. Le français est une langue appréciée, la langue d'un pays de rêve, d'exotisme occidental représenté par le parfum, la gastronomie et l'art selon la perception communément acceptée.

2. Kachru (1985) avait avancé le paradigme des cercles par rapport à la présence de l'anglais. Le « cercle grandissant » se réfère à la présence de la langue dans les pays où l'anglais n'est ni la langue maternelle de la majorité (cercle interne) ni une langue officielle (cercle externe). Ce paradigme a lui-même été critiqué pour avoir créé des distinctions hiérarchiques concernant les langues. 
Cette réception du français accorde à l'enseignant une certaine sécurité provenant du prestige de la langue et confie au locuteur de cette langue et à l'enseignant, un statut privilégié, d'apanage, d'admiration. L'identité de l'enseignant indien de FLE s'articule autour de ce rapport particulier avec la langue française parmi d'autres éléments.

\section{Méthodologie}

L'étude cherche à valoriser les représentations des enseignants, leurs expériences et leurs émotions concernant la langue française. Une étude qualitative avec quinze enseignants indiens de FLE a été menée. Des entretiens semi-dirigés ont été effectués avec des enseignants de tranches d'âge différentes (entre 27 et 62 ans, dont trois hommes et douze femmes), d'établissements de travail différents, possédant des qualifications et expériences professionnelles variées. Trois enseignants étaient munis d'un doctorat en français, cinq d'un master et sept ne possédaient pas de diplôme universitaire en français mais avaient un niveau B1-B2. Les années d'expérience s'étalaient entre 39 années d'expérience (pour l'enseignante la plus expérimentée) et 3 ans (pour l'enseignante la moins expérimentée). Tous les enseignants enquêtés étaient plurilingues, connaissant au moins deux langues en dehors de leur langue première et le français. Les questions portaient notamment sur le rapport à la langue française, les insécurités vécues par les enseignants, les causes et les stratégies pour les vaincre, ainsi que sur la confiance et la sécurité ressenties par les enseignants. Le tableau récapitulatif ci-dessous présente la répartition des personnes interrogées selon le type d'établissement, leurs qualifications, leur âge, leur expérience professionnelle et les langues qu'elles connaissent. 
Schéma 1 : Détails du corpus

\begin{tabular}{|c|c|c|c|c|c|c|}
\hline No & $\begin{array}{c}\text { Enseignant } \\
\text { (E) }\end{array}$ & Age & Qualifications & $\begin{array}{c}\text { Type } \\
\text { d'établissement }\end{array}$ & $\begin{array}{c}\text { Années } \\
\text { d'expérience }\end{array}$ & $\begin{array}{l}\text { Langues } \\
\text { connues }\end{array}$ \\
\hline & $F$ & 42 & Doctorat & Université & 15 & $\begin{array}{l}\text { anglais, français, } \\
\text { tamoul (L1), hindi }\end{array}$ \\
\hline & $\mathrm{H}$ & 35 & Doctorat & $\begin{array}{l}\text { École/Alliance } \\
\text { Française }\end{array}$ & 13 & $\begin{array}{c}\text { anglais, français, ta- } \\
\text { moul, télougou (L1), } \\
\text { hindi }\end{array}$ \\
\hline & $\mathrm{F}$ & 60 & Doctorat & $\begin{array}{l}\text { Université } \\
\text { (retraitée) }\end{array}$ & 30 & $\begin{array}{l}\text { anglais, français, } \\
\text { tamoul (L1), hindi }\end{array}$ \\
\hline & $\mathrm{F}$ & 27 & Master (français) & $\begin{array}{c}\text { Université/Alliance } \\
\text { Française }\end{array}$ & 6 & $\begin{array}{l}\text { anglais, français, } \\
\text { marathi }(L 1) \text {, hindi }\end{array}$ \\
\hline & $\mathrm{F}$ & 27 & Master (français) & Université & 3 & $\begin{array}{c}\text { anglais, français, } \\
\text { marathi }(L 1) \text {, hindi, } \\
\text { italien }\end{array}$ \\
\hline & $\mathrm{F}$ & 28 & Master (français) & École & 8 & $\begin{array}{c}\text { anglais, français, ma- } \\
\text { rathi, konkani (L1), } \\
\text { hindi }\end{array}$ \\
\hline & $\mathrm{F}$ & 43 & Master (français) & École & 15 & $\begin{array}{l}\text { anglais, français, } \\
\text { marathi (L1), hindi }\end{array}$ \\
\hline & $F$ & 62 & Master (français) & Université & 39 & $\begin{array}{c}\text { anglais, français, } \\
\text { marathi, hindi (L1), } \\
\text { ourdou }\end{array}$ \\
\hline & $F$ & 57 & $\begin{array}{l}\text { Licence } \\
\text { (économie) }\end{array}$ & École & 22 & $\begin{array}{c}\text { anglais, français, } \\
\text { goudjrati (L1), hindi, } \\
\text { marathi }\end{array}$ \\
\hline & $F$ & 28 & $\begin{array}{c}\text { Licence } \\
\text { (commerce) }\end{array}$ & École & 8 & $\begin{array}{c}\text { anglais, français, } \\
\text { goudjrati (L1), hindi, } \\
\text { marathi }\end{array}$ \\
\hline & $\mathrm{H}$ & 27 & $\begin{array}{c}\text { Licence } \\
\text { (commerce) }\end{array}$ & Cours particuliers & 8 & $\begin{array}{c}\text { anglais }(\mathrm{LI}) \text {, français, } \\
\text { marathi, hindi }\end{array}$ \\
\hline & $\mathrm{H}$ & 40 & $\begin{array}{c}\text { Licence } \\
\text { (commerce) } \\
\end{array}$ & Cours particuliers & 19 & $\begin{array}{l}\text { anglais, français, } \\
\text { goudjrati (L1), hindi }\end{array}$ \\
\hline & $\mathrm{F}$ & 54 & $\begin{array}{c}\text { Licence } \\
\text { (commerce) }\end{array}$ & Cours particuliers & 12 & $\begin{array}{l}\text { anglais, français, } \\
\text { tamoul (L1), hindi }\end{array}$ \\
\hline & $\mathrm{F}$ & 53 & $\begin{array}{c}\text { Licence } \\
\text { (économie) }\end{array}$ & Cours particuliers & 13 & $\begin{array}{l}\text { anglais, français, } \\
\text { punjabi (L1), hindi }\end{array}$ \\
\hline & $\mathrm{F}$ & 30 & $\begin{array}{c}\text { Licence } \\
\text { (commerce) }\end{array}$ & Cours particuliers & 7 & $\begin{array}{l}\text { anglais, français, } \\
\text { hindi (L1), marathi }\end{array}$ \\
\hline
\end{tabular}




\section{Résultats}

Les lacunes et les déficits perçus par les enseignants concernant leurs compétences linguistiques ainsi que le sentiment de confiance et de sécurité dont les enseignants font part se manifestent sous des signes différents, que nous analysons dans les paragraphes qui suivent. Les propos illustrent le rapport d'ambivalence des enseignants entre la sécurité linguistique et l'insécurité linguistique par rapport à la langue française. Nous verrons que l'insécurité linguistique se manifeste dans l'acte d'enseignement, les contextes en dehors de l'enseignement (à savoir les évaluations par les pairs ou les locuteurs natifs) ainsi que dans les stratégies employées par les enseignants.

\subsection{L'enseignement}

L'acte d'enseignement est le contexte le plus important où le rapport à la langue se fait évident. Alors que la préparation en amont donne une certaine confiance, rien ne prépare l'enseignant aux questions imprévues devant lesquelles il risque de se retrouver sans réponse.

C'est très stressant. Il y a quelques étudiants qui sont très intelligents et quand on ne connaît pas la réponse, c'est un peu difficile. (E11)

Il y a toujours un peu de stress - des mots que je ne connais pas, des phrases... (E12)

Je n'ai pas de peur quand je vais en classe, car on se prépare à l'avance, on prépare bien. Mais c'est vrai que quelquefois on est coincés - on ne connaît pas le mot, ou le sens d'un mot. Alors je dis aux étudiants - je ne peux pas tout savoir. Je vais vérifier ça. Il faut être honnête. (E1)

En raison de l'invisible hiérarchie qui régit en classe, l'insécurité reste limitée ou absente, surtout quand les apprenants sont jeunes.

Je donne des cours aux enfants jusqu'à l'âge de 11 ans. Il y a le problème de la discipline, mais je n'ai pas de difficultés autrement. (E6)

Je n'ai pas peur de ma grammaire ou mes connaissances en classe car mon niveau est meilleur que les étudiants, ça va, donc. (E12)

Par contre avec les apprenants plus âgés et d'un niveau plus élevé, la situation d'enseignement peut devenir plus anxiogènes.

Il faut que je me prépare bien. Certains étudiants sont très intelligents et aussi quelquefois un peu audacieux (E4) 
Aujourd'hui, les étudiants ont accès à l'internet, ils savent beaucoup. Ils posent des questions difficiles quelquefois et demandent le vocabulaire pour beaucoup de choses. Je n'ai pas la réponse à tout. Je dois aussi améliorer mes connaissances et j'essaie de le faire. (E2)

En classe, ils se sentent en sécurité par rapport à la hiérarchie supérieure devant les apprenants, le pouvoir de diriger le cours et la parole et la préparation en amont, qui laisse un meilleur degré de contrôle. Ces éléments deviennent des stratégies pour combler l'insécurité linguistique. Ce déficit linguistique est parfois source d'insécurité et de malaise, mais quelquefois les enseignants acceptent la situation et savent l'analyser sans culpabilité ou insuffisance. Cela est surtout perceptible chez les enseignants qui ont dépassé les cinquante ans.

À l'époque, nous n'avions pas l'occasion de parler et de pratiquer la langue. Mais aujourd'hui les moyens sont très variés. Mes étudiants parlent mieux que moi quelquefois ! C'est intéressant. (E9)

Quand on a appris le français, on faisait beaucoup de grammaire. On ne parlait pas vraiment. Et il n'y avait pas de possibilités. Aujourd'hui c'est différent car les étudiants peuvent regarder les films français, rencontrer des Français. À l'école, ils apprennent à parler aussi. J'apprends avec eux, moi aussi ! (E3)

Je donne des cours privés aux écoliers des écoles internationales. Souvent j'ai des élèves brillants, ils sont vraiment bien et certains prononcent mieux que moi. Ils sont superbes. (E14)

On constate que l'insécurité linguistique ici, même si elle existe, n'est pas toujours néfaste et se transforme en sentiment positif, celle d'appréciation pour les apprenants.

\subsection{Les qualifications, la formation, l'expérience}

Les diplômes contribuent à la sécurité linguistique des enseignants, tout comme le manque de diplôme est susceptible de créer de l'anxiété concernant la compétence linguistique. La mobilité vers la France augmente le sentiment de confiance et aide à mitiger l'insécurité linguistique.

J'ai fait des études supérieures, un master et puis mon doctorat d'une université en France. J'ai beaucoup travaillé et je travaille pour mes cours aussi. Ça me donne de la confiance. (E2)

J'ai passé un an en France lorsque j'étais assistante d'anglais. Je pouvais bien travailler là-bas. Je comprenais les Français et ils me comprenaient bien. Je crois que je parle bien, ça fait beaucoup d'années que je travaille avec la langue française maintenant. (E1)

J'ai fait plusieurs stages de formation en Inde et en France. Ça a beaucoup enrichi ma façon de travailler, de parler, d'enseigner. (E3) 
Je n'ai pas fait un MA ou un doctorat en France. Il y a longtemps j'ai fait mon diplôme de langue à l'Alliance Française et puis j'essaie de garder le contact avec la langue. (E13)

De même, les stages de formation et la participation aux événements pédagogiques renforcent la confiance et aident l'enseignant à s'évaluer de manière équilibrée.

Je vais à certains séminaires avec mes amis. Je fais mon travail, je connais mes faiblesses. J'aime enseigner et je fais mon travail honnêtement. (E14)

Les stages, les séminaires sont très utiles. J'apprends beaucoup de choses et j'améliore mon niveau. (E7)

Il y a souvent des événements - séminaires, ateliers, formations. J'assiste quand je peux, assez souvent. C'est très important pour moi - rester en contact avec la langue, avec les techniques d'enseignement et enrichir mes compétences et mon français aussi. (E12)

Je vois que j'ai encore beaucoup à apprendre. Donc je me dis, oui, ça va, je vais m’améliorer. (E6)

Les enseignants avec un diplôme élevé (master ou doctorat en français) se montrent plus confiants. Les stages de formation renouvellent la confiance en la matière et la prise de conscience des déficits langagiers écarte l'insécurité. La mobilité vers la France est également un facteur enrichissant.

Si la compétence linguistique est primordiale, celle-ci n'est pas pérenne. L'attrition a un impact direct sur la compétence linguistique.

Lorsque j'étais en France, j'avais l'opportunité de parler la langue tous les jours et donc je parlais bien et j'avais beaucoup de confiance. Mais maintenant, j'hésite un peu quelquefois. (E1)

Quand j'étais étudiante à l'université, on organisait des événements, mais maintenant, dans cette petite ville, j'ai très peu d'interactions, et peu d'occasions de pratiquer mon français. Je n'ai plus la même aisance qu'avant. C'est pas bien! (E3)

Après la retraite, je continue à enseigner, mais ce n'est pas comme avant, vous voyez. Les occasions sont limitées maintenant de pratiquer la langue, et puis automatiquement, on cherche des mots, des expressions etc., car on n'utilise pas la langue tous les jours. C'est un peu stressant. (E8)

La langue évolue, le verlan, le français parlé... il faut être constamment en contact avec la langue. C'est un voyage de découverte. Sinon, on va trainer et ça crée des angoisses. (E9)

Une modification peut s'effectuer dans la compétence linguistique, engendrée par plusieurs causes, ce qui crée plus d'insécurité ou moins d'insécurité selon les situations. 


\subsection{Les attentes, l'évaluation des autres, l'autoévaluation}

Les enseignants diplômés ont en général une bonne confiance et ne dévoilent aucune insécurité linguistique dans leur discours. Mais certains dérogent à cette norme. Comme ces enseignants qui possèdent un doctorat mais qui remettent en question leur compétence dans la langue.

Je n'ai pas l'accent parfait français. Je ne parle pas très vite non plus. Il faut faire attention surtout quand on parle devant les Français. Ça me gêne. (E3)

Oui, quand j'enseigne la littérature, j'ai un peu peur - est-ce que je suis capable de m'exprimer bien en français, bien communiquer les idées littéraires? (E1)

Le malaise devant la langue cible vient des attentes ambitieuses et difficiles que les enseignants s'imposent à eux-mêmes.

Il est important de bien parler, surtout quand on est prof. (E15)

Quand j'écoute les Français, je me dis - je dois parler comme ça. (E11)

Parler bien, parler rapidement, faire un discours - comme les Français! Oui, c'est un rêve! (E12)

La peur du jugement et les attentes des autres peuvent être très porteurs et sont mis en avant par les enseignants.

Quand on assiste aux séminaires, j'hésite à parler en français. Il y a des collègues qui parlent mieux. Je sais que je n’ai pas un très bon niveau à l'oral. (E9)

Un professeur doit être bien, on attend ça de l'enseignant. Et ce n'est pas toujours facile. (E7)

Aujourd'hui les étudiants ont beaucoup d'ouverture. Ils souhaitent avoir des professeurs qui parlent bien, qui prononcent bien. Une fois, un étudiant a dit - ce mot il faut le prononcer comme ça, pas comme ça... (E13)

Les parents aussi sont très ambitieux pour leurs enfants - ils nous posent des questions sur notre qualification, notre niveau. Tout cela met de la pression - le professeur doit être bien, parler bien, prononcer bien... (E14)

La pression, l'envie et l'impossibilité d'arriver à cette échelle, est également source d'angoisse et augmente le sentiment d'insuffisance, de dévalorisation et d'incapacité. Ils sont confiants devant les apprenants, mais peu confiants devant les locuteurs natifs ou d'autres collègues. Il s'agit aussi d'une insécurité liée à la production et à la réception également. 
Quand on parle aux Français, je dois faire très attention pour ne pas faire d'erreurs, pour tout comprendre aussi. Il faut rester très attentif. Même si on parle bien, parler avec les Français c'est différent. (E1)

Quand je parle aux Français, j'hésite quelquefois. J'ai peur de faire des erreurs de grammaire. (E5)

Les Français parlent très vite et je ne comprends pas tout. C'est difficile pour moi. (E9)

Je parle bien. Mais devant les Français, je suis stressée. J'hésite beaucoup, mes phrases sont coupées. (E13)

Après tout c'est la langue des Français, pour moi c'est une langue étrangère. Je fais des fautes et quelquefois je ne comprends pas tout ce qu'ils disent. Ce n'est pas possible, ils parlent vite. (E11)

Quand on parle aux Français - quelquefois on n'a pas assez de vocabulaire - il faut faire attention. (E15)

Imiter le natif et parler comme les natifs, c'est toujours le modèle, le but n'est-ce pas ? Ce n'est pas toujours possible mais il faut l'essayer quand même. (E8)

L'anxiété de l'évaluation négative par les autres est évidente dans ces propos. Cette situation est comparable à ce que Cho (2015) évoque au sujet des interprètes coréens de l'anglais, qui malgré leur bonne maitrise de l'anglais, ressentent une insécurité surtout lorsqu'ils se comparent aux locuteurs natifs. Donc l'insécurité linguistique n'est pas uniquement associée à la maitrise linguistique. L'autoévaluation s'avère aussi exigeante en ce qui concerne l'estimation des compétences. Horwitz (1996) évoque l'analyse parfois irrationnelle des compétences de la part du locuteur de langue étrangère, qui mène à une situation anxiogène. Les chercheurs soulignent aussi que les perfectionnistes, qui se fixent un haut niveau de performance, ont tendance à se sous-évaluer, aboutissant à une anxiété. Prenons cet exemple d'une enseignante possédant un master en français et six ans d'expérience. Un élément d'infériorité implicite et sous-jacent se révèle dans ce discours : « Je ne suis pas sûre de bien parler, parfois je pense que le niveau est insuffisant. » (E4)

Malgré un niveau linguistique appréciable, quelques enseignants ont tendance à se comparer à leurs pairs qu'ils estiment meilleurs qu'eux, et également à se comparer - ce qui est paradoxal - avec les natifs. Par conséquent, ils s'évaluent négativement, engendrant une infériorité de soi concernant le français (v. Meyerhoff, 2006). Cette enseignante, par exemple, compare son français à son niveau en anglais: 
Mon insuffisance en français devient plus forte car j'ai tendance à comparer ma maîtrise en français à mon niveau en anglais. Je possède une très bonne maîtrise de la langue anglaise et quelque part la différence est visible pour moi. Ce que je possède en anglais, ce que je ne possède pas en français, vous voyez? (E1)

Il s'agit d'une perception de compétence-incompétence liée à l'autoévaluation sévère, non associée aux qualifications ou à l'expérience, touchant les plus expérimentés et les novices en même temps. Un sentiment d'insécurité devant les pairs perçus comme plus compétents peut également engendrer un sentiment d'exclusion chez certains qui restent donc « en marge » de la communauté enseignante, même s'ils ne l'expriment pas ouvertement.

Nous nous rencontrons de temps en temps, on discute un peu en français, on parle de nos défis, les étudiants, les manuels. Une possibilité de discuter - actually. (E15)

Très souvent, nous organisons des réunions informelles entre nous. Nous discutons en anglais mais on discute à propos de notre travail, les jeux qu'on emploie avec nos étudiants, les films sur Youtube qu'on peut montrer aux étudiants. (E9)

Nous avons un groupe de «tuition teachers» (enseignants de cours particuliers). Nous discutons, nous sortons, j'apprends des choses. (E14)

Ces réunions communautaires entre pairs confèrent une sécurité aux enseignants, écartant les malaises qu'ils ressentent vis-à-vis de la langue enseignée et leurs pratiques.

L'âge des enseignants a une influence sur les représentations qu'ils ont de leurs compétences. Les enseignants de moins de 40 ans, avec un niveau élevé mais sans diplôme en français, se montrent confiants: « Non, je ne m'inquiète pas, je parle bien », « Non, je n'ai aucune angoisse ou de difficulté par rapport à la langue. » Par contre, les enseignants plus âgés portent un avis différent par rapport aux jeunes - «plus confiants » (E3), « très confiants » (E8), « changement de valeurs » (E9), « les jeunes sont moins exigeants, ont plus de confiance» (E14), « ce n’est pas la même génération » (E1).

Les représentations qu'ont les enseignants de leurs compétences peuvent aussi être erronées. Il peuty avoir une surestimation comme une sous-estimation des compétences. Si les lacunes perçues peuvent créer une situation anxiogène et laisser l'enseignant démuni, de même une estimation « irrationnelle » peut aussi créer des situations de confiance, aveuglant les vrais déficits linguistiques. La sous-évaluation comme la surévaluation sont des notions intéressantes à analyser. Les études montrent que la surévaluation est liée à une compréhension et une formation insuffisantes du domaine concerné et une amélioration des compétences peut mener à une autoévaluation plus exacte (Kruger et Dunning, 1999). La sous-évaluation des compétences provient entre autres de la pression professionnelle et du désir d'imiter l'idéal linguistique, qui semble difficile à atteindre. Si l'on s'en tient aux propos des enseignants, on remarque que ceux qui se sous-estiment sont freinés non par leur manque de compétence linguistique mais plutôt par la perception de leurs compétences. 


\subsection{Le rôle de l'établissement}

Les enseignants de cette étude exercent dans différentes structures - cours particuliers, écoles, lycées, Alliances Françaises, universités, chaque structure ayant sa renommée et son prestige. Les cours privés, par exemple, n'ont pas de légitimité institutionnelle et n'occupent pas le même statut dans l'échelon professionnel. Les enseignants sans diplôme universitaire en français et qui exercent en privé souffrent d'une double exclusion qui les dévalorise et semble augmenter le sentiment d'insuffisance.

Les étudiants ont beaucoup d'attentes, je dois donc toujours renouveler ce que je sais. J'assiste aux stages et j'utilise l'internet pour lire des informations en France, des vidéos, les documentaires. (E11)

Comme je ne travaille pas dans une école ou un collège, je me sens assez seule et quand j'ai la possibilité de faire de formations, je le fais. Oui, je me sens un peu démoralisée mais l'attente des apprenants semble jouer un rôle aussi. (E13)

Ma grammaire est très bien. Mais je n'ai pas l'occasion de pratiquer l'oral. Les étudiants veulent faire des exercices et avoir une bonne note à l'examen. Donc mon français parlé n'est pas très bien. (E9)

Mon école est un établissement assez réputé. Nous avons des étudiants intelligents, curieux. Je peux vraiment essayer des activités différentes en français avec eux. C'est beaucoup de travail mais c'est un plaisir. (E6)

J'ai fait un diplôme de langue il y a longtemps. Mais mes étudiants me permettent de rester en contact car j'enseigne le syllabus international et ça donne l'occasion de pratiquer mon français. Je n'ai pas pu faire un diplôme, mais je crois que je suis en contact constant avec le français, donc mon niveau de langue aussi est assez bien grâce à ça. (E14)

Nous avons beaucoup d'examens et des copies à corriger. Et aussi beaucoup de - comment on dit - portions ? Beaucoup de « portions » à finir. Je veux parler plus couramment. Mais je n’ai pas le temps pour améliorer cela. (E7)

J'enseigne à la fois au collège-université et à l'Alliance Française. Par exemple à l'Alliance Française le travail est très différent et on a l'occasion de parler en français. Et les étudiants y vont par plaisir. Ici, c'est un choix imposé ou presque. Et peu d'occasions de pratiquer l'oral. (E4)

L'établissement confie une légitimité et par là une confiance. Travailler dans une école réputée ou une université confère une sécurité linguistique que le travail à son compte n’accorde pas toujours. Même si nous n'avons pas inclus l'observation de cours dans notre démarche méthodologique, les 
enseignants parlent de leur pratique qui subit les influences de l'insécurité linguistique. Ceux qui sont mal à l'aise avec l'oral ont tendance à limiter les interactions orales en classe: « Nous n'avons pas le temps pour l'oral », « les étudiants ne sont pas intéressés », « les examens, les examens. II faut tout finir pour préparer l'examen. Où est le temps?»

Ces justifications pour limiter l'oral en classe peuvent être tout à fait légitimes dans la situation indienne. En même temps, l'angoisse devant cette composante risque de freiner sa place dans les cours. Le sentiment de «ne pas être à la hauteur » se cache dans les discours. Nous pouvons donc émettre l'hypothèse que la qualité de l'enseignement et sa spontanéité risquent de se trouver compromises, lorsque l'insécurité linguistique intervient.

\subsection{Alterner les langues}

Lors de l'entretien, presque tous les enseignants (à l'exception d'un enseignant) utilisaient l'anglais et quelques mots de hindi.

Je crois que quelquefois, je n'ai pas la... J'oublie le mot... command over the language. Et j'utilise l'anglais. (E12)

Can I speak in English ? I would be more comfortable. (E15)

Je peux parler en français aussi, mais je préfère l'anglais. (E9)

Ici, l'alternance de langues est révélatrice d'un manque de compétence communicative perçue ainsi et révélée par l'enseignant. On pourrait l'attribuer à une proximité de la langue de tous les jours et par moments un déficit dans la langue cible. La plupart des enseignants ne peuvent donner un cours uniquement en français - cela ne répond ni aux besoins des étudiants, ni au système institutionnel, souvent axé sur le cursus et l'examen. Cette situation d'enseignement, cause de l'insécurité linguistique en même temps, se présente comme une stratégie pour combler les déficits linguistiques.

Parfois j'enseigne en tamoul aussi. Nous avons des étudiants qui viennent des couches sociales peu privilégiées et c'est nécessaire d'utiliser leur langue pour qu'ils comprennent. Donc je n'ai pas l'occasion vraiment de beaucoup parler en français en classe. (E1)

En classe, ce n'est pas possible d'utiliser uniquement le français, les étudiants ne comprendraient pas. (E5)

Ma langue préférée - je peux le dire ? J'ai le droit de le dire dans cet entretien ?! C'est l'anglais. J'aime lire et parler en anglais, je suis proche de l'anglais. (E1)

Quand j'enseigne, j'utilise l'anglais et le français - les deux. Comme ça les étudiants n'ont pas de difficulté. (E7) 
Nous avons un système éducatif où l'écrit est important. Donc il n'y vraiment pas le temps pour pratiquer le français parlé en classe. Mais j'utilise quelques phrases en anglais et en français. (E6)

Alterner les langues, même s'il est ordonné par la situation d'apprentissage, fait partie intégrante du répertoire linguistique des enseignants. Pour Macaro (2005) il s'agit d'une stratégie de compensation pour combler le déficit linguistique, notamment dans une situation d'enseignement. Cela aide aussi à dissimuler leur compétence communicative supposée limitée (Agudo, 2017).

\subsection{Sécurité, confiance et intimité avec la langue}

Enseigner une langue étrangère confère aux enseignants un prestige dans la société et rapproche l'enseignant de la langue enseignée.

Les gens apprécient que j'enseigne une langue étrangère. (E11)

Dans ma famille, mes amis, ils trouvent que c'est quelque chose de différent. Je suis contente d'avoir choisi ce métier. (E9)

Quelquefois j'écris des poèmes en français. Quand j'ai du temps je lis des livres aussi en français. J'adore ça. (E3)

S’ils ressentent une insécurité par rapport à la langue, ils partagent une intimité avec la langue aussi.

J'adore le français. Cette langue est importante pour moi. (E15)

La France est le premier pays étranger que j’ai visité, et c'est grâce à la langue française. Cette langue est spéciale dans ma vie. (E6)

Je fais le français depuis très longtemps. J'ai une relation très spéciale avec cette langue. (E2)

J'écoute Renaud, Souchon, Cabrel, les chansons interprétées de Louis Aragon... Je lis. Le lien avec cette langue est quand même intime avec presque 39 ans d'enseignement ! Le plaisir du savoir, le sens de l'esthétique, tout est lié à cette langue. C'est une découverte sans fin. (E8)

Ces témoignages nous rapprochent de la recherche réputée de Pavlenko (2005) sur les « embodied languages » (langues incarnées). La chercheure montre la proximité et la forte charge émotionnelle des langues qui font partie de la trajectoire linguistique du locuteur, à savoir, les langues « embodied » ou « incarnées », notamment les langues premières. Même si le français n'est pas la langue première pour les enseignants indiens et n'a pas suivi le même parcours que la L1, cette langue peut quand même prendre la place d'une langue « incarnée » et certains enseignants construisent un lien 
affectif fort avec le français comme on le remarque dans cette étude, par l'ensemble des expériences - le pays, des expériences vécues en France, des amis francophones, des films français, des chansons, les écrivains etc.

\section{Discussion}

\subsection{Une insécurité linguistique protéiforme}

Nous remarquons, chez les enseignants, un tiraillement entre la norme (le français « correct» des livres et celui propagé par l'idéal des modèles des locuteurs natifs, des pairs plus diplômés, mieux formés, ou possédant plus de maitrise sur le plan langagier selon les enseignants), une question d'identité (le désir de démontrer une maîtrise linguistique se rapprochant de cet idéal) et l'envie de se prouver professionnellement (le désir de se projeter comme étant capable et à la hauteur comme enseignant). Il s'agit du défi de faire une carrière d'une langue qui est la sienne et en même temps qui ne l'est pas, des valeurs culturelles et interculturelles propagées par la langue qui ne sont pas toujours faciles à appréhender, et de se créer une identité professionnelle et personnelle entre cette langue et les autres qu'ils connaissent déjà et probablement avec une meilleure maîtrise.

Ainsi, l'enseignant « non natif » subit-il des affrontements et des difficultés sur plusieurs plans, certains plus durs à négocier que d'autres, certains créés par les représentations, les attitudes et attentes de la part de la société et d'autres par une auto-évaluation sévère. On peut donc évoquer une « violence symbolique » (Derivry-Plard et Griffin, 2017), perceptible et imperceptible, mais qui influe sur l'identité et le discours enseignant. Dans certains cas, l'âge, les établissements de travail, les diplômes, les formations professionnalisantes contribuent aux sentiments de sécurité-insécurité. Ceux-ci ne sont pas uniquement liés à la maîtrise de la langue, comme nous l'avons vu, car les enseignants possédant un bon niveau peuvent s'estimer encore faibles linguistiquement tout comme ceux qui ont besoin de s'améliorer linguistiquement ne ressentent pas d'insécurité. La distinction établie par Moreau entre l'insécurité dite - « telle que la reflètent les discours épilinguistiques » - et l'insécurité agie - «celle qui transpire dans les pratiques » (Moreau, 1996: 159) - est ici pertinente. Nous remarquons que les enseignants indiens démontrent plus «l'insécurité dite » par rapport à «l'insécurité agie ».

\subsection{La sécurité-insécurité : un processus évolutif}

Nous constatons que la sécurité-insécurité linguistique n'est pas un phénomène figé. Elle est en constante évolution, impactée par des facteurs comme par exemple l'attrition individuelle, le travail actif, les lieux d'activité professionnelle, les diplômes. La restructuration des compétences linguistiques reste toujours une possibilité, car les acquisitions sont évolutives, liées en partie au contact avec la langue cible et l'enrichissement des compétences linguistiques. Parfois les enseignants sont 
susceptibles de renier l'insécurité linguistique pour « sauver la face ». Dans d'autres cas, l'alternance des langues et la participation aux actions de formation s'avèrent utiles pour surmonter l'insécurité linguistique. Il s'agit donc souvent d'une insécurité linguistique dynamique (Ledegen, 2000), qui permet d'améliorer les pratiques langagières.

L'insécurité linguistique, si elle existe, n'est pas toujours cause d'angoisse. Certains enseignants voient leurs faiblesses linguistiques comme un défi à surmonter afin de monter en grade; d'autres se démotivent. Plus de la moitié des enseignants ont démontré une perception favorable d'eux-mêmes. L'expérience professionnelle à l'étranger et la mobilité vers la France pour des études ou des stages servent également de facteurs importants vis-à-vis de l'évolution des représentations sur la sécurité linguistique.

Les retrouvailles « en communautés » (dont l'utilité a été soulignée par Wenger dès les années 1990), entre enseignants, servent à accroître leur bien-être et écarter l'insécurité linguistique. La stratégie de la communauté de pratique rejoint la notion d'« attachement à la langue », avancée par VidrineIsbell (2017). Empruntant ses idées à la neurolinguistique, la chercheure s'en sert dans le contexte de l'acquisition d'une langue seconde. Le locuteur s'attache à la langue et développe un rapport spécial, grâce à la présence d'une personne qui aide à l'apprentissage, à soulager l'anxiété et donner du plaisir dans l'apprentissage. Par ce processus, l'autre devient la figure de l'attachement à la langue, un facteur pédagogique d'apprentissage. Comme l'explique Vidrine-Isbell (2017: 110), il ne s'agit plus d'un expert qui aide le non-expert. Les deux sont désormais « experts » (en L1) et locuteurs de L2 dans un acte d'entraide. Une communauté de pratique joue le même rôle sécurisant et réconfortant, se situant davantage dans un paradigme de partage.

\subsection{L'idéal du natif}

Même si Paikeday (1985) avait fameusement déclaré que « le locuteur natif est mort », annonçant un tournant dans les perspectives, nous sommes encore loin de ce projet, trois décennies plus tard. Tous les enseignants de cette étude, sans exception, sont irréductiblement penchés vers le modèle natif. Ils ont tous évoqué la notion du « locuteur natif » comme modèle à suivre, un idéal intériorisé, qui en soi leur est valorisant. S'associer à l'idéal « natif » n'est pas forcément une distinction négative pour les enseignants indiens car celle-ci est valorisante et donne indirectement une légitimité à l'enseignant (Badrinathan, 2007) et l'absence de cette distinction pourrait lui priver de l'idéal à atteindre, et par là d'une comparaison valorisante (Dervin et Badrinathan, 2011: 14). Ayant dit cela, il est néanmoins impératif de faire évoluer ces représentations, afin d'avancer vers l'idéal du locuteur plurilingue comme préconise le CECRL, utile pour encourager l'apprenant à apprécier son plurilinguisme sans influence négative (Zheng, 2017). 


\subsection{Un plurilinguisme présent mais « absent »}

Tous les enseignants sont au moins trilingues, mais l'absence de valorisation de cette compétence est frappante. Cette situation n'est pas surprenante, comme une étude antérieure le révèle (Badrinathan et Leconte, 2018). Une identité plurilingue pourrait se présenter comme panacée à l'enseignant « non natif » vis-à-vis de son rapport avec la langue enseignée. Reconnaître les compétences plurilingues pourrait induire une nouvelle dimension identitaire d'enseignant de langue étrangère, celle d'un locuteur pluricompétent (Cook, 2012) et non d'un «failed speaker » ou locuteur peu performant d'une langue cible (Pavlenko, 2003). Pour ce faire, un changement des représentations chez les enseignants et une modification des perspectives seraient nécessaires. D'où l'indispensable place du plurilinguisme dans les formations d'enseignants.

\subsection{Un rapport et une posture indéfinissables}

Chez la plupart des enseignants interviewés, le français dépassait sa seule fonction utilitaire. La langue joue plusieurs rôles - fonctionnel, utilitaire, affectif, identitaire - combinant paradoxalement l'insécurité et la confiance. L'insécurité due au déficit linguistique que les enseignants ressentent parfois, ne limite pas pour autant la proximité spéciale qu'ils entretiennent avec cette langue. La problématique de la sécurité-insécurité linguistique des locuteurs d'une langue est liée à ce qu'expliquent succinctement Bretegnier et Ledegen. Les enseignants indiens de FLE se retrouvent entre plusieurs «frontières », plusieurs enjeux - «frontières de langues, de normes, de groupes, frontières entre ce qui est dans la norme et ce qui en est exclu, entre le légitime et l'illégitime, entre le Même et l'Autre, entre l'intérieur et l'extérieur» (Bretegnier et Ledegen, 2002 : 12).

\section{Remarques conclusives}

L'intérêt de cette étude réside dans les éclairages qu'elle apporte sur le rapport à la langue et la dimension affective des enseignants indiens de FLE. Plus particulièrement, l'insécurité linguistique, qui est souvent négligée, cachée ou ignorée dans les discours est ici étudiée. L'insécurité-sécurité linguistique se présente comme un sujet en mouvement, complexe, définie et redéfinie par plusieurs facteurs y compris l'âge, les qualifications, l'expérience, l'établissement de travail. Ce qui ressort de cette étude est que l'insécurité linguistique porte uniquement sur la communication orale. Seule la production orale et la réception de la parole contribuent à l'insécurité linguistique et sont sources d'anxiété, notamment par manque d'occasions de pratique orale. Toutefois, en Inde, l'enseignement du français est souvent axé sur l'écrit au détriment de son aspect communicatif. Cet enseignement-apprentissage behaviouriste et prescriptif est à son tour susceptible de renforcer le poids de l'insécurité linguistique. Par ailleurs, nous ne voyons aucune valorisation des langues locales ou indiennes que possèdent les enseignants. La dimension du locuteur plurilingue pourrait renforcer 
les compétences, servir à diminuer la vulnérabilité que l'insécurité linguistique risque d'apporter, et remanier l'identité de l'enseignant indien de FLE.

Cette étude démontre que l'insécurité linguistique est compensée par plusieurs stratégies employées consciemment ou inconsciemment par les enseignants. Ces stratégies mettent en évidence la capacité à transformer les éléments négatifs en éléments positifs afin de surmonter le problème en question. Parmi les stratégies, celle des communautés de pratique s'avère intéressante. Les communautés de pratique promeuvent l'activité collaborative, et présentent l'enseignant en tant qu'acteur social en lien avec les approches socioconstructivistes et actionnelles. Les communautés de pratique pourraient être rendues légitimes par leur insertion dans des dispositifs de formation, démocratisant ainsi la formation et le métier. Au cœur de cette théorie réside l'importance du relationnel humain qui sécurise l'apprentissage, renforçant à nouveau le côté affectif qui est indissociable de l'apprentissage des langues. Elles serviraient aussi à écarter les frustrations, les obstacles, à atténuer non seulement les insécurités et les anxiétés mais également apprendre à s'autoévaluer de manière équilibrée.

Finalement, nous avons constaté que les enseignants indiens de FLE se positionnent dans ce « tiers-espace » dont avait parlé Bhabha (1994), qui est à la fois un privilège et un défi à affronter. Par la nature même de l'enseignement d'une langue étrangère, l'enseignant est confronté à un « autre » espace culturel, linguistique et identitaire où il est appelé à rencontrer à la fois la langue étrangère et soi-même (Péréa, 1997). S'il existe des insécurités liées à la langue, qui sont presque inévitables, cela n'exclut pas le rapport d'intimité avec la langue et la fierté d'enseignant, ce qui en soi devient sécurisant: ces deux positions ne sont donc pas incompatibles, comme cette étude le montre. Cela explique également le rapport ambivalent des enseignants indiens à la langue cible, caractérisé par ces dualités.

Cette étude, aussi modeste qu'elle soit, est importante dans la mesure où elle est probablement l'une des premières à porter sur la dimension affective des enseignants indiens de FLE en Inde et notamment l'insécurité-sécurité linguistique, sujet souvent laissé à la marge. Il est important de problématiser la dimension affective, la reconnaître, afin de créer des situations d'enseignement-apprentissage stables et équilibrées. La présente étude mérite d'être approfondie, notamment en interrogeant la corrélation entre l'insécurité linguistique des enseignants de FLE et les pratiques de classe. 


\section{Références}

Agudo, Juan de Dios Martínez (2017), « Non-native teachers' code-switching in L2 classroom discourse », dans Juan de Dios Martínez Agudo (dir.), Native and Non-native Teachers in English Language Classrooms: Professional Challenges and Teacher Education, Boston/ Berlin, De Gruyter Mouton, p. 75-98.

Badrinathan, Vasumathi (2007), Identités professionnelles et autonomie des enseignants non natifs de FLE : le cas de l'Inde, thèse de doctorat, Lille, Université de Lille 3.

Badrinathan, Vasumathi et Fabienne Leconte (2018), «Plurilinguisme indien et représentations des enseignants de FLE », Glottopol, n³0 (Le plurilinguisme en contextes asiatiques : dynamiques et articulations, sous la dir. de Fabienne Leconte, Vasumathi Badrinathan et Gilles Forlot), p. 81-102.

Bhabha, Homi (1994), The Location of Culture. Londres/New York, Routledge.

Blommaert, Jan, James Collins et Stef Slembrouck (2005), «Spaces of multilingualism », Language and Communication, $n^{\circ}$ 25, p.197-216.

Bretegnier, Aude et Gudrun Ledegen (2002), Sécurité, insécurité linguistique, Paris/La Réunion, L'Harmattan/Université de la Réunion.

Bucci, Wilma et Milton Baxter (1984), "Problems in linguistic insecurity in multicultural speech contexts », Annals of the New York Academy of Sciences, n 433, p.185-200.

Conseil de l'Europe (2011), Cadre européen commun de référence pour les langues, Strasbourg, Conseil de l'Europe.

Castellotti, Véronique (2011), « Natif, non natif ou plurilingue : dénativiser l'enseignement des langues? », dans Fred Dervin et Vasumathi Badrinathan (dir.), L'enseignant non-natif : identités et légitimité dans l'enseignement-apprentissage des langues étrangère, Bruxelles, Éditions modulaires européennes, p. 29-50.

Cho, Jinhyun (2015), « Sleepless in Seoul: Neoliberalism, English fever, and linguistic insecurity among Korean interpreters », Multilingua, vol. 34, n5 5. p. 687-710.

Cook, Vivian (2012), « Multicompetence », dans Carol Chapelle (dir.), The encyclopaedia of applied linguistics, Oxford, Wiley-Blackwell, p. 1127-1131.

Daftari, Giti Ehtesham (2016), « A Study of Linguistic Insecurity among Turkish Teachers of English », International Journal of English Language Education, n 4, p. 117-130.

Derivry-Plard, Martine et Claire Griffin (2017), «Beyond Symbolic Violence in ELT in France », dans Juan de Dios Martínez Agudo (dir.), Native and Non-native Teachers in English Language Classroom : Professional Challenges and Teacher Education, Boston/Berlin, Walter de Gruyter, p. 33-51.

Dervin, Fred et Vasumathi Badrinathan (2011), L'enseignant non-natif: identités et légitimité dans l'enseignement-apprentissage des langues étrangère, Bruxelles, Éditions modulaires euroépennes. 
Dewaele, Jean-Marc (2018), «Why the Dichotomy "L1 Versus LX User" is Better than "Native Versus Non-native Speaker” », Applied Linguistics, vol. 39, n², p.236-240.

Gueunier, Nicole, Émile Genouvrier et Abdelhamid Khomsi (1978), Les Français devant la norme, Paris, Honoré Champion.

Harada, Sanae (2019), « Repenser la dichotomie entre enseignants natifs et non-natifs à travers le numérique : le cas du Japon », ELA : Études de linguistique appliquée, nº 193, p. 107-118.

Horwitz, Elaine, Michael Horwitz et Joanne Cope (1986), « Foreign Language Classroom Anxiety », The Modern Language Journal, vol. 70, n², p. 125-132.

Horwitz, Elaine (1996), «Even teachers get the blues: Recognizing and alleviating non-native teachers' feelings of foreign language anxiety », Foreign Language Annals, vol. 29, n³, p. 365-372.

Houghton, Stephanie et Kayoko Hashimoto (2018) (dir.), Towards post-native-speakerism, Singapore, Springer.

Houghton, Stephanie et Jérôme Bouchard (2020) (dir.), Native Speakerism, Singapore, Springer.

Kachru, Braj (1985), «Standards, codification, and sociolinguistic realism : The English language in the outer circle », dans Randolph Quirk et Henry Widdowson (dir.), English in the World: Teaching and Learning the language and the literature, Cambridge, Cambridge University Press, p. 11-30.

Kruger, Justin et David Dunning (1999), « Unskilled and Unaware of It: How Difficulties in Recognizing One's Own Incompetence Lead to Inflated Self-Assessments », Journal of Personality and Social Psychology, vol. 77, nº, p. 1121-1134.

Labov, William (2006), The social stratification of English in New York City, $2^{\mathrm{e}}$ éd., New York, Cambridge University Press. [1éd., 1966.]

Ledegen, Gudrun (2000), Le bon français : Les étudiants et la norme linguistique, Paris, L'Harmattan.

Llurda, Enric (2006), Non native language teachers, New York, Springer.

Macaro, Ernesto (2005), « Code switching in the L2 classroom : a communication and learning strategy », dans Enric Llurda (dir.), Non-native Language teachers, New York, Springer, p. 63-84.

Medgyes, Peter (1992), « Native or non native : Who's worth more? », ELT Journal, vol. 46, n 4, p. 340349.

Meyerhoff, Miriam (2006), Introducing Sociolinguistics, New York, Routledge.

Mikyoung, Lee, Paul Schutz et Stephen van Vlack (2017), « Non-native English-speaking teachers’ anxieties and insecurities: Self-perceptions of their communicative limitations », dans Juan de Dios Martínez Agudo (dir.), Native and Non-Native Teachers in English Language Classrooms, Boston/ Berlin, De Gruyter Mouton, p 119-138. 
Moreau, Marie-Louise (1996), «Insécurité linguistique: pourrions-nous être plus ambitieux? Réflexions au départ de données camerounaises, sénégalaises et zaïroises », dans Claudine Bavoux (dir.), Français régionaux et insécurité linguistique, Paris, L’Harmattan, p. 103-114.

Mousavi, Elham Sadat (2007), « Exploring "teacher stress" in non-native and native teachers of EFL », English Language Teacher Education \& Development, n 10, p. 33-41.

Paikeday, Thomas (1985), The Native Speaker is Dead!, Toronto, Paikeday Press. Pavlenko, Aneta (2003), « "I never knew I was bilingual" : Reimagining Teacher Identities in TESOL », Journal of Language, Identity and Education, vol. 2, n 4, p. 251-268.

Pavlenko, Aneta (2005), Emotions and multilingualism, Cambridge, Cambridge University Press.

Péréa, François (1997), «Quand Marco devient Marc: Quelques rencontres affectives dans l'entre deux langues », Travaux de didactique du FLE, n 38, p. 251-267.

Preston, Dennis (2013), « Linguistic insecurity forty years later », Journal of English linguistics, vol. 41, n० 4, p. 304-331.

Rappa, Antonio L. et Lionel Wee (2006), Language policy and modernity in Southeast Asia, New York, Springer Science +Business Media.

Rogers, Carl (1965), «Psychology and Teacher Training », dans Gowan D.B. and Richardson C. (dir.), Five Fields and Teacher Education, Ithaca, Project One Publications.

Rogers, Carl, (1967), "The Interpersonal Relationship in the Facilitation of Learning ", dans Robert Leeper (dir.), Humanizing Education: The Person in the Process, Washington, Association for Supervision and Curriculum Development, p.1-18.

Roussi, Maria et Kenza Cherkaoui Messin (2011), « L'insécurité linguistique entre pratiques enseignantes et compétences langagières : vers une redéfinition du rôle de l'enseignant de langues », dans Fred Dervin et Vasumathi Badrinathan (dir.), L'enseignant non-natif: identités et légitimité dans l'enseignement-apprentissage des langues étrangère, Bruxelles, Éditions modulaires européennes, p. 237-264.

Rubdy, Rani (2001), "Creative destruction: Singapore's speak good English movement », World Englishes,vol. 20, n³, p. 341-355.

Schutz, Paul, et Mikyung Lee (2014), «Teacher emotion, emotional labor and teacher identity », dans Juan de Dios Martínez Agudo (dir.), English as a Foreign Language Teacher Education : Current Perspectives and Challenges, Amsterdam/New York, Rodopi, p. 169-186.

Stevick, Earl (1980), Teaching Languages : A Way and Ways, Rowley, Newbury House.

Vidrine-Isbell, Bonnie (2017), «Language attachment theory: The possibilities of cross-language relationships », dans Patricia Portanova, Michael Rifenburg et Duan Roen (dir.), Contemporary perspectives on cognition and writing, Fort Collins, The WAC, p. 95-114.

Wenger, Étienne (1998), Communities of practice: Learning, meaning and identity, Cambridge, Cambridge University Press. 
Zheng, Huang (2018), Native and Non-Native English Speaking Teachers in China, Singapore, Springer.

Zheng, Xuan (2017), «Translingual identity as pedagogy: international teaching assistants of English in college composition classrooms », Modern Language Journal, vol. 101, n 51, p. 29 -44. 


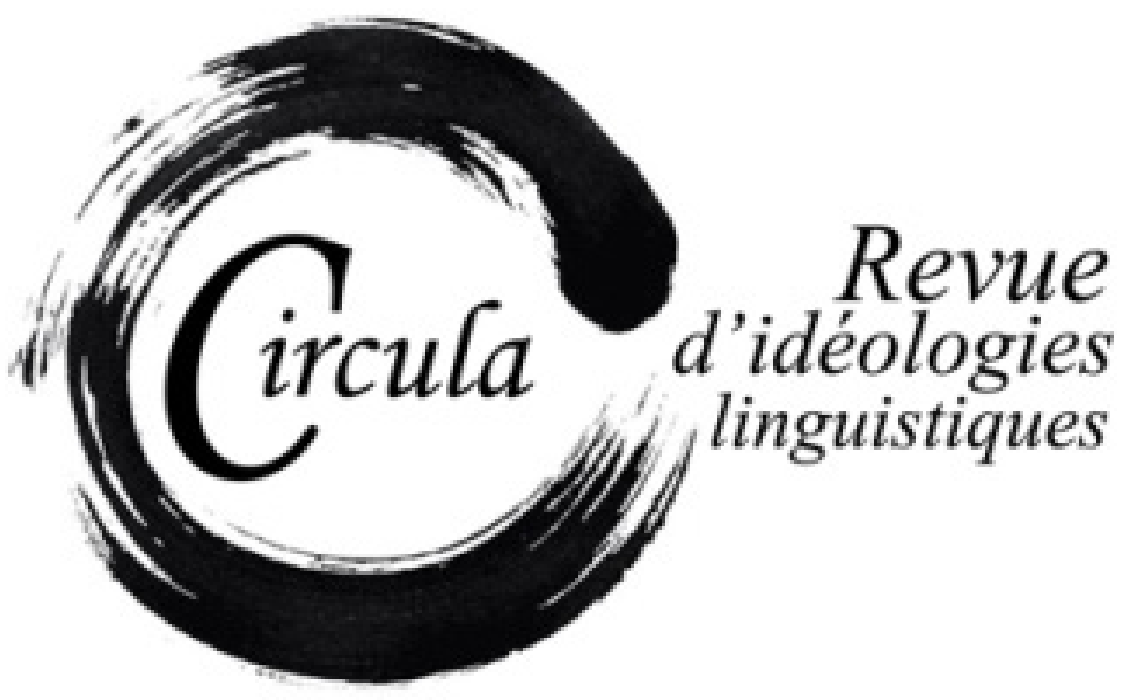

TITRE: INSÉCURITÉ LINGUISTIQUE CHEZ LES ENSEIGNANTS NON NATIFS DE FLE : LE CAS DES COLOMBIENS Auteur: Claudia Rincón Restrepo, École Jeannine Manuel- Paris, France

ReVue: Circula, NumÉro 12 : LA VULNÉRABILITÉ LINGUISTIQUE

DiRECTRICE: CLAUDIA TORRES CASTILLO

PAGES: $176-196$

ISSN: 2369-6761

URI: HTTP://HDL.HANDLE.NET/11143/18448

DOI: HTTPS://DOI.ORG/10.17118/11143/18448 


\title{
Insécurité linguistique chez les enseignants non natifs de FLE : le cas des Colombiens
}

\author{
Claudia Rincón Restrepo, École Jeannine Manuel- Paris, France \\ c.rinconrestrepo@ejm.net
}

\begin{abstract}
Résumé : Cet article s'intéresse à la notion d'insécurité linguistique dans les processus d'enseignement des langues étrangères, notamment celle ressentie par les enseignants de français non natifs en Colombie. Nous allons donc analyser les possibles raisons pour lesquelles les professeurs de français langue étrangère (FLE) colombiens ressentent cette insécurité, tout en abordant la notion et la représentation que se font ces enseignants du locuteur natif / non natif, ainsi que celles de langue maternelle ou première (LM/L1), qui en sont très proches. Nous présenterons également le statut et la place de la langue française en Colombie, avant d'évoquer le rôle joué par la formation des professeurs de FLE assurée dans les universités colombiennes. Enfin, nous présenterons quelques propositions pour tenter de remédier à ce sentiment d'insécurité, et améliorer ainsi non seulement la qualité de la formation et du travail des professeurs de FLE, mais aussi leur vie en général.
\end{abstract}

Mots clés : enseignement de FLE ; locuteur natif/non natif; insécurité linguistique

\begin{abstract}
This article examines the notion of linguistic insecurity in the process of learning/teaching foreign languages, in particular that felt by non-native French teachers in Colombia. We will therefore focus on the possible reasons why Colombian teachers of French as a foreign language (FLE) feel this insecurity, while presenting the notion and representation that these teachers make of the native / non-native speaker, as well as of the mother tongue or first langue (LM/L1), both being very close. We will also explore the status and place of the French language in Colombia, and finally discuss the role played by the training provided in Colombian universities for future language teachers. Finally, we will make some suggestions to address this feeling of insecurity and thus improve not only the quality of the training and the work of FLE teachers, but also their lives in general.
\end{abstract}

Keywords: teaching of FLE; native/non native speaker; linguistic insecurity 


\section{Introduction}

L'insécurité linguistique est un sujet largement discuté et étudié depuis l'apparition du terme dans les années 60. Pour le professeur de langue étrangère (LE) non natif, cette insécurité peut nuire à son développement professionnel, voire personnel. Elle peut avoir différentes origines, personnelles comme sociales, liées principalement aux représentations ou images que les enseignants et les professeurs des langues se font d'eux-mêmes et des autres, du processus cognitif et du modèle à suivre pour parvenir à «maitriser » une LE. Cette vulnérabilité linguistique parait s'accroitre en raison du sentiment d'illégitimité par rapport au professeur natif, ainsi qu'à l'idée de souffrir d'un déficit linguistique dû à un manque de pratique de la langue. Par ailleurs, le sentiment de ne pas suffisamment connaitre la culture de la langue cible, le fait d'avoir une prononciation incorrecte ou un accent très marqué et, enfin, le sentiment d'avoir reçu une formation médiocre peuvent également jouer un rôle important. Ayant identifié ces situations générant de l'insécurité chez les enseignants, comment y remédier?

\section{L'insécurité linguistique}

La notion d'insécurité linguistique apparait avec Labov en 1964. Haugen est pourtant le premier à utiliser ce terme, en $1962^{1}$, pour analyser l'insécurité dans des situations linguistiques où deux variétés d'une même langue coexistent. Il la décrit comme l'image négative ou le sentiment de dépréciation de soi-même ressenti par un locuteur exposé à une variété de sa propre langue plus valorisée que la sienne. Labov développe l'idée de Haugen (1962) et montre l'importance des facteurs extralinguistiques pour expliquer ce qui est linguistique. Ses études sur l'insécurité se centrent principalement sur la catégorisation sociale dans un même espace linguistique (l'anglais). D'après ses études, les pratiques les plus valorisées sont celles utilisées par l'élite (économique, politique, culturelle), qui s'imposent comme la norme officielle. Le prestige dont elles sont dotées n'a rien à voir avec leurs qualités intrinsèques, mais s'explique par celui dont sont investis les locuteurs de cette variété de langue comme norme sociale.

Plus tard, Francard, Lambert et Berdal-Masuy (1993: 13) théorisent cette notion à partir d'une étude sur des cas de diglossie en Belgique. Pour lui, l'insécurité linguistique est « la manifestation d'une quête non réussie de légitimité » et dépend de la prise de conscience du locuteur de la distance entre son idiolecte et une langue qu'il reconnait comme légitime, normalement celle apprise lors de la scolarisation. L'instruction donnerait en effet la connaissance de la norme sans pour autant assurer la maitrise pratique des registres, ce qui crée de l'insécurité.

1. Haugen parle de schizoglossie et de la norme linguistique. 


\subsection{L'insécurité linguistique en LE}

Calvet (1993) analyse les phénomènes relatifs à cette insécurité d'un point de vue inter-linguistique, c'est-à-dire en tenant compte de diverses situations de plurilinguisme. D’après lui, cette vulnérabilité apparait lorsque les locuteurs considèrent leur façon de parler, notamment dans les aspects phonétiques et syntaxiques, comme peu valorisante par rapport à un autre modèle considéré plus prestigieux qu'ils ne pratiquent pas. Il propose trois types d’insécurité linguistique:

- l'insécurité identitaire, qui apparait lorsque la langue ou variété pratiquée par le locuteur ne correspond pas à la communauté linguistique à laquelle il appartient ou à celle qu'il désire intégrer;

- l'insécurité formelle, liée au fait que le locuteur considère sa propre pratique linguistique comme non-conforme aux normes, ou à l'idée qu'il se fait de celles-ci ;

- l'insécurité statutaire, qui nait lorsque la langue ou variété pratiquée par le locuteur est perçue comme illégitime ou n'a aucun statut reconnu.

Selon Bourdieu dans son livre Ce que parler veut dire : l'économie des échanges linguistiques, la vulnérabilité linguistique chez les personnes s'exprimant dans une langue qui leur est étrangère surgit d'une non-maitrise des normes² ou de l'idée de ne pas les maitriser, et est liée à une volonté de parvenir à la forme considérée comme plus prestigieuse, ce qui peut amener à commettre des fautes et des erreurs.

Quand un locuteur se trouve dans une communauté linguistique où est pratiquée une langue qu'il ne maitrise pas, le sentiment d'insécurité linguistique se traduit chez lui par un effort conscient de correction afin de se rapprocher de l'usage jugé prestigieux. (Bourdieu, $1982: 104)$

Ainsi, les échanges entre un locuteur dit « natif ${ }^{3}$ » et un locuteur dit « non natif » peuvent entrainer des rapports de force qui, continuellement reproduits, constituent une forme de violence symbolique et qui, outre l'insécurité linguistique, peuvent mener à des représentations de soi extrêmement négatives.

2. Suivre la norme signifie respecter toutes les règles qu'une variété linguistique impose pour que l'usage soit correct pour celui qui veut l'imiter, l'utiliser ou la parler. Ces normes peuvent être perspectives, fictives, communicationnelles ou évaluatives.

3. Les ternes « natif » et « non natif » seront mis entre guillemets par la suite pour souligner le caractère relatif de ces notions. 


\subsection{Distinction entre locuteur «natif » et «non natif »: une source d'insécurité}

La figure du locuteur « natif » représente, de manière ambivalente, un fantasme et une source d'insécurité linguistique. Mais pourquoi le locuteur natif représente-t-il un idéal à suivre?

La notion de locuteur «natif » est introduite par la linguistique traditionnelle structurale. Celle-ci oppose de façon irréductible au sein de chaque langue le locuteur « natif » au locuteur « non natif ». Selon l'affirmation chomskyenne, l'objet de la linguistique serait ce natif, présenté comme un :

Ideal speaker-listener, in a completely homogeneous speech-community, who knows its language perfectly and is unaffected by such grammatically irrelevant conditions as memory limitations, distractions, shifts of attention and interest, and errors (random or characteristic) in applying his knowledge of the language in actual performance. (Chomsky, $1965: 3$, cité par Muni Toke, 2010 : 54)

De son coté, Love (2009 : 28), définit les locuteurs natifs comme «those who have been born and brought up to speak [a language] from birth, as their "mother tongue", in a family where the parents or other adults were brought up to speak the language from birth ».

Toutefois, pour Derivry-Plard, il s'agit de distinguer ce qui dans les notions de « natif » et « non natif » appartient à la catégorisation linguistique et à la construction sociale. En effet, la notion définie par Chomsky est, pour elle, une abstraction théorique où l'on voit la langue comme un système normatif et non comme une variation Dans son article Les enseignants «natifs » et «non-natifs » de langue(s): catégorisation linguistique ou construction sociale?, elle écrit:

Cette notion de «locuteur natif » a été particulièrement centrale pour l'élaboration de la grammaire générative développée par N. Chomsky. Il s'agissait de s'appuyer sur les intuitions grammaticales, lexicales et stylistiques d'un locuteur natif idéal, (c'est-à-dire aussi éduqué et «monolingue ») pour rendre compte du système de la langue décrit et de son fonctionnement comme capacité à générer un nombre illimité d'énoncés grammaticalement corrects ou acceptables à partir d'un nombre limité de signes. (...). La linguistique variationniste va s'opposer radicalement à cette vision «normative » de la langue, en remettant en question ces notions mêmes de «langue » et de «locuteur » comme définitions étanches et en les posant toutes deux selon des continua et un principe de diversité ; dans un même espace linguistique, il y a une diversité de langues (orales, écrites qui varient selon les âges, le sexe, l'origine sociale et géographique des divers locuteurs et selon les situations langagières et selon aussi que la compétence du locuteur diffère également de celle de l'auditeur). (Derivry-Plard, $2006: 101)$. 
Comme le dit cette auteure, la linguistique variationniste et plus encore la sociolinguistique, vont s'opposer à cette définition étanche et à cette vision structurale de la langue, remettant en question cette notion, et même celle de langue. Cette figure de locuteur natif sera aussi abondamment discutée en linguistique théorique, suivant ainsi le courant des études postcoloniales, dénonçant dans cette acception une position occidentalo-centralisée de la science linguistique, comme le montrent les travaux de Coulmas (1981) ou plus récemment de Davies (2003).

Dans son texte, Paikeday (1985) considère que cette notion de « natif » s'apparente davantage à un mythe propagé par les linguistes. D’autres chercheurs, comme par exemple Rampton (1990), proposent de remplacer ce terme par celui de locuteur expert, car ce dernier fait référence à l'expérience de locuteur dans une langue et non pas à son identité. Cook (2016), pour sa part, préfère le terme locuteur multi compétent, faisant référence aux niveaux de maitrise atteints dans différentes langues.

Le terme LM, étroitement lié à celui de locuteur natif, a également été remis en question, notamment par Urbain (1982: 8). Celui-ci considère que ce concept ne se laisse pas appréhender aisément et peut être défini de façons diverses. Pourrait-on alors lui donner un sens unique?

Du latin maternus, ce terme apparait pour la première fois au Moyen Âge en référence à la langue régionale parlée dans des contextes informels, en opposition à l'usage érudit du latin. Actuellement, il désigne la première langue apprise par un enfant à la maison de manière inconsciente, ce que l'on a souvent compris comme étant la langue de la mère. Par cette langue maternelle, l'enfant apprend la maitrise pratique des règles d'interaction sociale dans un ensemble culturel donné. Ces définitions ne sont cependant pas suffisantes pour couvrir toutes les réalités socio linguistiques existantes, car la langue de la mère n'est pas toujours la première langue apprise. Dans le cas de couples mixtes ou de milieux plurilingues, par exemple, elle peut être la langue véhiculaire ou commune entre les parents, la langue du père ou d'autres.

Pour tenir compte de ces ambiguïtés et confusions, la didactique des langues préfère l'usage de L1 (langue première) plutôt que maternelle. Davies (2003 : 16-17) observe ainsi qu'un enfant peut acquérir deux ou plusieurs langues simultanément. On peut ainsi dire qu'un enfant peut avoir deux ou plusieurs LM, mais il serait pourtant difficile d'établir laquelle est sa L1. D'autres chercheurs, comme Skutnabb-Kangas et McCarty (2018), définissent la LM selon quatre critères : l'ordre d'apprentissage (première langue apprise), l'identification (langue(s) avec la(es)quelle(s) le locuteur s'identifie ou est identifié), la compétence (la(es) langue(s) qu'il maitrise le mieux) et la fonction (la(es) langue(s) la(es) plus utilisée(s)). Ainsi, la ou les L1 d'un individu peuvent changer au cours de son existence, selon un principe évolutif et non statique.

Selon Dabène (1994) dans Torres-Castillo (2018 : 92-93), la langue maternelle est « celle dans laquelle s'est organisée la fonction langagière elle-même, en tant que fonction symbolique primordiale, et celle qui accompagne la construction de la personnalité ». D'après cette auteure, diverses considérations doivent être prises en compte pour définir la notion de LM, qui ne serait pas seulement 
la langue de la mère comme le dit l'étymologie. Elle peut être la première langue dans l'ordre d'acquisition, c'est-à-dire juste après la naissance, d'où les expressions fréquentes de « langue native » et de «locuteur natif ». La LM peut également désigner la langue la mieux connue par le locuteur, s'il en parle plusieurs, ou celle qu'il a apprise «naturellement ». Ceci signifie trois choses : on apprend cette langue sans s'en rendre compte, il n'existe pas d'intervention pédagogique quelconque et l'on l'apprend en parlant avec les autres.

\subsection{Distinction entre locuteur «natif » et professeur «natif »}

Derivry-Plard (2008 : 190) affirme que locuteur et professeur de langue ne doivent pas être confondus, car un locuteur est rarement enseignant de sa LM, et un professeur de LE est toujours un «natif » dans une ou plusieurs langues. Un «natif » peut en effet être très compétent dans l'usage d'une langue, mais ne pas avoir les compétences nécessaires pour l'enseigner. Le locuteur « natif » fait plutôt référence à une catégorisation linguistique quelque peu dépassée, alors que le terme « enseignant » fait référence à une catégorie sociale professionnelle dont les membres disposent tous, en droit, de la même compétence d'enseignement. Dans sa thèse de doctorat (2003), cette auteure expose les conclusions d'une analyse quantitative, comparative et multivariée comparant les résultats d'étudiants apprenant une LE avec un professeur « natif », et d'autres apprenant avec un «non natif ». Il apparait que les seconds ont obtenu de meilleurs résultats que les premiers, invalidant l'idée que l'enseignement d'un professeur «natif » est meilleur que celui d'un «non natif».

Malgré ces résultats gratifiants et encourageants, les professeurs « non natifs » se sentent toujours vulnérables, illégitimes. Derivry-Plard explique par exemple qu'en France les enseignants d'anglais non natifs ressentent de l'insécurité car les apprenants préfèrent avoir des professeurs natifs. Cela reflète une attitude générale et largement partagée dans l'opinion française. Dans les domaines économique, médiatique, politique et éducatif existe un consensus selon lequel un professeur natif serait plus compétent qu'un non natif.

D’après Coste (2008, cité par Torres-Castillo, 2017 : 142), le modèle sur lequel se basent les niveaux de compétence des enseignants en LE a été construit à partir de la figure du locuteur natif. C'est ce locuteur natif qui sait prononcer correctement les mots, a une vaste connaissance lexicale et connait très bien sa culture. Dans l'imaginaire des enseignants et des apprenants, ce «natif » est le locuteur idéal et légitime et parle une langue standard. À ce propos, Muni Toke nous dit : 
L'idéalisation chomskyenne, en faisant de la variation linguistique une quantité négligeable dont le scientifique devrait se défaire, aurait contribué à renforcer la centralité du locuteur natif et de la langue standard dans les sciences du langage. Or, ces deux notions sont des idéologies politiques, associées à la discrimination ethnique et à l'homogénéisation forcée des pratiques linguistiques; pourtant, la définition chomskyenne est apolitique, décontextualisée, sans aucune dimension sociale et sociologique. (...) On pourra reprocher à cet enchainement apparemment logique la confusion de deux ordres : celui du natif, description commune à valeur sociale et politique, et celui de l'idéal, modélisation scientifique explicitement « réductionniste » et « extractionniste». (Muni Toke, 2013:8)

Cet amalgame entre locuteur « natif » et « idéal » relève donc d'une confusion, d'une construction sociale et non d'une réalité. Il faudrait, comme le disent Candelier et Castellotti (2013), disqualifier cette notion dans le domaine de l'enseignement des langues, car il n'existe pas un seul modèle de locuteur natif. Selon Muni Toke:

Ce terme «natif » est également porteur d'une valeur ethnico raciale implicite : l'authenticité et la légitimité du locuteur natif tiennent à l'appartenance culturelle qu'on lui prête - voire à son phénotype comme dans l'exemple ci-dessus. On voit quels raccourcis il a soudainement fallu accepter, pour que la « compétence » supposée d'un locuteur se retrouve corrélée à son origine ethnique. Et pourtant, la construction d'inégalités sociales à partir de ce présupposé est monnaie courante, et il y aurait bien ainsi des natifs qui seraient plus natifs que d'autres. (Muni Toke, 2003 : 6)

\section{L'insécurité chez l'enseignant de FLE « non natif »}

Tant dans son entourage que dans son milieu professionnel, l'enseignant de LE est considéré comme un modèle, compétent ou capable non seulement d'enseigner, mais aussi de communiquer sans problème, de répondre, de poser des questions et de discuter en utilisant la langue étrangère dans laquelle il a suivi une bonne formation et a obtenu un diplôme universitaire. L'image que son entourage a de ce professeur expert influe sur sa sécurité. Il est difficile pour un enseignant, voire honteux, de confesser qu'il rencontre des difficultés dues à l'insécurité linguistique, qui, comme dit plus haut, peuvent avoir des origines diverses. Nous évoquerons ici celles relatives à l'insécurité identitaire évoquée par Calvet (1993), liée à la conscience de ne pas être « natif » et aux images que les enseignants/apprenants se font de ce professeur « natif », et à l'insécurité formelle, qui peut provenir du sentiment de ne pas maitriser les normes en raison d'un manque de pratique ou d'une formation insuffisante.

Concernant l'insécurité linguistique chez les enseignants « non natifs » de LE, Roussi (2009 : 21) écrit, dans sa thèse de doctorat, que leur conscience normative serait partagée entre la langue qu'ils 
ont apprise, qu'ils enseignent avec les fautes, les imperfections ou tout simplement les particularités des locuteurs « non natifs » et la perception stéréotypée d’un locuteur « natif » supposé idéal.

Dans la tradition de l'enseignement des LE et dans l'opinion publique, la distinction, voire hiérarchisation, entre locuteur « natif » et « non natif » a toujours été présente. On peut même parler d'une opposition entre ces deux concepts, voire d'une confrontation (Planet, 2014). Cette idée s'est renforcée depuis l'apogée de la méthode directe à la fin du XIX` siècle et au début du XX siècle, où l'exercice privilégié était la conversation en LE sans devoir passer par la langue maternelle et où le professeur ne devait pas donner d'explication sur le fonctionnement de la langue. Durant cette période, n'importe quel locuteur natif pouvait en effet devenir professeur de LE, l'objectif étant simplement de parler et d'apparaitre aux apprenants comme un modèle à suivre.

Bien que cette méthode ne soit plus en vigueur, la figure du professeur de LE « natif » reste, dans divers contextes, insubstituable et idéale : il connait sa propre culture et maitrise sa LM, prononce correctement les mots, est authentique, légitime et fiable.

Roussi (2009) aborde l'insécurité linguistique vécue par les professeurs de FLE en Grèce comme un sentiment de déficit linguistique, de peur et de honte de commettre des erreurs pendant leurs cours, ou de reconnaitre qu'ils manquent de connaissances linguistiques et culturelles. Ils ressentent cette insécurité non seulement dans le cadre académique, mais aussi en dehors de la salle de classe, par exemple dans les échanges de la vie quotidienne avec des natifs ou des collègues. Dans la salle de classe, le professeur développe des stratégies d'évitement qui peuvent l'aider à contourner les difficultés. En revanche, dans la vie réelle, quand la communication n'est pas guidée, le fait de mal prononcer un mot, de l'oublier ou de l'utiliser de manière incorrecte, peut être ressenti comme une mise en défaut de sa maitrise de la langue, et de ses compétences en général. Cette vulnérabilité peut donc affecter non seulement sa vie professionnelle, mais aussi sa vie privée.

L'exemple des professeurs grecs s'apparente, là aussi, à l'expérience des enseignants en Colombie, qui peut devenir tellement stressante que certains professeurs partent en France ou dans un pays francophone, en quête de légitimité4. Ils veulent poursuivre leurs études et ainsi revendiquer leur compétence en tant que professeurs de FLE, avec l'espoir de revenir plus crédibles au pays. D'autres, une fois installés dans un pays francophone, se tournent plutôt vers l'enseignement de leur propre L1 - l'espagnol - dans lequel ils se sentent plus reconnus et légitimes.

Pour Roussi, le manque de pratique, en dehors du contexte scolaire, de la langue enseignée peut être une autre source d'insécurité pour les professeurs. La situation est similaire en Colombie, en raison du statut du français dans le pays. La possibilité de parler cette langue en dehors d'un contexte académique est en pratique très faible. La situation s'est toutefois améliorée ces dernières années grâce aux programmes d'échanges académiques entre la France et la Colombie, l'arrivée massive

4. Ainsi, 56\% des 3000 Colombiens étudiant en France sont dans des filières linguistiques. 
des nouvelles technologies et l'ouverture du pays au tourisme international, après avoir été isolé par plus de 50 ans de conflit armé.

\subsection{L'insécurité linguistique chez les enseignants « non natifs » de FLE en Colombie}

En Colombie, l'opposition entre « natif » et « non natif » perdure, principalement dans le milieu académique (Rincón Restrepo, 2016 :327), créant ainsi une différence de statut entre ces deux profils, ainsi qu'un sentiment d’illégitimité et de vulnérabilité chez les enseignants « non natifs » de FLE. Ceux-ci éprouvent alors des difficultés à se positionner vis-à-vis du professeur francophone natif. Ils ont le sentiment, de par leur perception propre et celle de leur entourage, que leur compétence sera toujours dévalorisée face à l'image parfaite du «natif ».

Dans l'enseignement des LE en Colombie, la progression des étudiants et aussi la compétence du personnel enseignant sont mesurées selon l'image du locuteur « natif » idéal. Ce qui a des répercutions parfois très négatives sur leur motivation, car les apprenants, en constatant qu'ils n'atteindront jamais le niveau d'un natif, peuvent se décourager. Comme le dit Torres-Castillo:

Les idées stéréotypées des langues et des locuteurs empêchent de comprendre l'enseignement-apprentissage des langues comme une relation des différentes connaissances linguistiques, et elles peuvent à la fois devenir de vrais empêchements pour la maitrise de la langue et la cause de diverses déceptions. (Torres-Castillo, 2018: 53)

Cette idéalisation du natif se voit parfois reflétée dans les offres d'emploi, car les institutions cherchent des «natifs » pour plus tard mieux vendre leurs services, renforçant ainsi l'idée stéréotypée du « natif » auprès de la population : lui seul peut enseigner sa culture ou comment bien prononcer sa langue, et il lui suffit pour cela d'être en contact avec ses étudiants, ce qui n'est pas réaliste. Cette situation, souvent vécue dans des pays comme la Colombie, où la plupart des professeurs de FLE sont « non natifs » (Colombiens) ${ }^{5}$, génère un énorme désavantage entre les professeurs formés dans les universités colombiennes ayant acquis des connaissances linguistiques et pédagogiques solides, et les natifs qui n'ont parfois même pas de formation en enseignement de leur LM. Le désavantage est, bien sûr, encore plus marqué si ceux-ci ont suivi une formation pédagogique et didactique en LE. Avoir un professeur « natif » est devenu un privilège dans ce pays sud-américain. Ils sont généralement mieux payés, même quand ils ont une formation plus médiocre. Dans cette échelle professionnelle, l'enseignant latino-américain subira, outre l'insécurité linguistique, une discrimination à l'emploi.

\footnotetext{
5. Si, d'après le ministère de l'Éducation nationale en Colombie, la majorité des professeurs de LE sont des Colombiens, la proportion de professeurs natifs varie selon le type d'établissement. Les écoles bilingues internationales recrutent principalement des directeurs et des enseignants d'origine étrangère, et offrent notoirement de meilleures rémunérations que les autres. Dans les écoles bilingues nationales, les enseignants sont tous bilingues mais principalement d'origine colombienne. Dans les écoles non bilingues, seuls les professeurs de LE sont bilingues, et en quasi-totalité des Colombiens formés dans les universités du pays. Information disponible en ligne sur www.mineducacion.gov.co/1759/ w3-article-364450.html?_noredirect=1.
} 
Une autre des principales sources d'insécurité linguistique chez les professeurs colombiens de FLE est le fait d'avoir un accent étranger, non natif, ce qui est souvent perçu, selon Cook (2014, cité par Torres-Castillo, 2017 : 142) comme un échec dans l'apprentissage de la LE. En effet, nous savons qu'acquérir un accent natif, bien que possible, est très difficile, principalement pour des personnes commençant à apprendre une LE à l'âge adulte, cas de la plupart des étudiants des licences de formation de futurs professeurs des langues en Colombie (au moins pour ce qui concerne le français).

\begin{abstract}
(...) un individu qui n'a pas appris une langue dans l'enfance et ne l'apprend qu'à l'âge adulte la parle rarement sans accent. La raison en est que son appareil phonatoire n'aura pas assimilé les mouvements qui permettent de produire correctement les sons d'une langue donnée. La production de sons de la langue maternelle s'inscrit dans des algorithmes moteurs mémorisés par l'appareil neuromusculaire. Elle privilégie certains muscles et en laisse d'autres en jachère. Ensuite cette mémoire du corps contrarie l'émission normale de la plupart de sons des autres langues. Ainsi est de beaucoup d'éléments de culture qui sont autant de répertoires d'action et de pensées permanents qui nous façonnent parce que nous les avons incorporés. (Warnier, 2004 : 10, cité par Torres-Castillo, 2017 : 143)
\end{abstract}

Mais le fait d'avoir un accent n'a rien à voir avec la qualification pédagogique, voire avec la compétence linguistique. Cette idée réduisant la capacité d'enseigner une langue à la question phonologique reproduit, d'une certaine façon, la dichotomie entre dominant (le natif) et dominé (le non natif), comme le dit Torres-Castillo (2018). Jusqu'à aujourd'hui, les professeurs de LE sont souvent classifiés par rapport à leur L1 et non par leurs capacités linguistiques et pédagogiques.

Avoir un accent n'est pas le seul complexe des professeurs colombiens de LE. Toute marque de L1 dans leurs discours peut être mal perçue. Pourtant d’après Galisson (1986 : 52), la LM est présente de manière visible ou invisible dans l'apprentissage d'une LE, est en fait la première référence, le fil conducteur, une piste universelle. Les études psycholinguistiques montrent que la L1 est indéniablement omniprésente dans le cerveau des apprenants d'une LE/L2. En fait, faire appel à sa LM lors de l'apprentissage d'une LE est une pratique naturelle conforme à toute situation de communication de contact des langues (Causa, 2002, cité par Benamar, 2014 : 139). Ainsi, la LM ne devrait pas être vue comme un obstacle ou exclue de l'acte pédagogique en LE, mais plutôt mise à profit dans le processus d'apprentissage en tant qu'outil.

Par ailleurs, dans l'imaginaire de beaucoup de Colombiens, les natifs de langue française ne sont pas seulement supérieurs concernant la prononciation correcte et l'enseignement du français, mais dans tous les domaines. Il existe en effet en Colombie - et dans la plupart des territoires jadis colonisés - un sentiment d'infériorité par rapport aux européens, pour des raisons historiques, comme le dit Torres Castillo dans sa thèse (2018) à propos de la situation des enseignants non natif de FLE au Mexique. Lors de la colonisation de ce pays, par exemple, les relations qui s'établirent entre les Espagnols et les Indigènes étaient des relations de pouvoir déséquilibrées. Les Espagnols, convaincus de leur supériorité raciale et culturelle, considéraient les Indigènes comme des êtres peu évolués. 
De leur côté, les Indigènes les considéraient aussi comme des êtres supérieurs qu'ils respectaient et reconnaissaient comme tels. L'idéalisation de l'étranger au Mexique, et dans tous les territoires colonisés par les occidentaux, n'est peut-être que le résultat de cette vieille coutume qui préparait les individus à respecter les dieux. Torres-Castillo nous dit:

Cet imaginaire s'est construit à partir des récits de la Conquête et à partir aussi de quelques documents indigènes comme les codex. Malheureusement l'histoire a été plutôt écrite la plupart du temps par ceux qui ont vaincu aux yeux du monde, nous laissant juste une version occidentale de cet évènement historique. (Torres-Castillo, 2018: 155)

Cette idéalisation de l'étranger est donc très ancrée dans les mentalités latino-américaines. Comme nous le savons, la société coloniale en Amérique latine était organisée par castas. Celle qui se trouvait en haut de l'échelle était celle des Espagnols et des criollos (fils des Espagnols nés en Amérique). Celles des natifs et des noirs se trouvaient tout en bas. Ainsi, par exemple, quand un individu de l'époque voulait poursuivre ses études universitaires pour se préparer à accéder à des postes administratifs, il devait d'abord démontrer qu'il était un honorabilis vir, purus ab omni macula sanguinis atque legitimis et natalibus descendens, c'est-à-dire un « homme honorable, pur de toute tache de sang et descendant de parents légitimes ». Cette «tache » de sang fait référence au métissage avec les noirs ou les indiens. Les groupes de privilégiés - les Blancs européens - craignaient en effet l'avancée progressive des droits du peuple et du pouvoir politique et économique que commençait à obtenir la population métisse. Ces préjugés d'impureté étaient très présents et persistent, incroyablement, même aujourd'hui et l'Européen est encore vu comme supérieur (Rincón Restrepo, 2016 : 25, 27). Plus tard, au cours du XVIIle siècle, apparait en Colombie une certaine idéalisation de la France et des Français. À cette époque, le français est la langue de l'élite, du monde civilisé. De fait, à cette époque, la société hispano-américaine, comme le monde entier, est divisée entre civilisés et barbares. Cette dichotomie rend possible et légitime le colonialisme européen, notamment en Afrique et en Asie. Les Européens, se considérant supérieurs aux autres peuples, entreprennent de civiliser les peuples natifs de ces continents. Ce qui n'est plus possible aux Amériques, d'abord en raison d'une volonté d'indépendance dans presque tous les territoires, acquise récemment avec beaucoup d'efforts, ensuite parce que les États-Unis, dirigés par le président James Monroe, établissent la doctrine Monroe ${ }^{6}$ en 1823, considérant toute nouvelle intrusion des États européens comme un acte d'agression requérant l'intervention des États-Unis, et enfin, car, en Amérique latine, l'élite, d'origine espagnole, cherche à civiliser ou à « européaniser » la population de la nouvelle nation, rejoignant ainsi l'objectif des Européens. Ainsi, cette dichotomie, rendue publique en Argentine et ultérieurement dans toute l'Amérique hispanique par Domingo Faustino Sarmiento dans son livre Facundo, est à l'origine de la bataille pour l'instruction publique qui se développe en Amérique latine au XIX'e siècle. L'élite criolla, descendant des Espagnols, voit la société européenne comme une société modèle, civilisée, tandis que celle des nouvelles nations américaines est vue comme barbare et inférieure. Elles doivent

6. La doctrine Monroe : elle a rendu fameuse la phrase L’Amérique pour les Américains, et détermine la politique extérieure des États-Unis. 
être civilisées coûte que coûte, grâce à l'éducation, et à travers une langue européenne. L'espagnol, étant la langue maternelle de l'élite et la plus répandue - mais pas forcément la plus parlée - sur le territoire colombien, est donc choisie comme langue nationale. Toutefois, les idées véhiculées en langue espagnole sont, pendant cette période, celles des Européens. L'élite criolla continue alors à regarder vers la France et l'Angleterre, idéal de culture et de progrès, summum de la civilisation (Rincón Restrepo, 2016: 50). La francophilie est donc très présente non seulement en Colombie mais aussi dans toute l'Amérique latine au XVIII siècle. La France a marqué l'histoire du pays et a été, jusque récemment, le modèle à suivre en matière éducative comme dans bien d'autres domaines. Au XIXe siècle, le gouvernement de Simón Bolívar, très influencé par les idées républicaines de la France, se tourne principalement vers ce pays en matière d'éducation et tente de copier ses modèles éducatifs. Le choix d'enseigner le français est, lui, le fruit de l'influence de la culture française depuis le XVIIe siècle. Le rôle de la langue française est, surtout, de cultiver et civiliser les jeunes gens de la nouvelle république. Pendant le XIXe siècle, la langue française reste très importante pour des raisons historiques, culturelles, et diplomatiques. Les idéaux républicains français se répandent d'abord dans plusieurs domaines, notamment juridique et éducatif, grâce aux élites libérales, qui écrivent et parlent couramment le français. Les programmes éducatifs et les lois instaurés suivent ainsi le modèle français et s'inspirent des idées du siècle des Lumières. Jusqu'à la première moitié du XX siècle le français est considéré comme langue de civilisation, et est la première langue étrangère dans le pays. Les Français, quant à eux, sont vus comme des gens cultivés, par opposition aux Colombiens, mettant en évidence la dichotomie civilisation/barbarie discutée plus haut. Nous constatons ainsi que la rencontre avec l'étranger, si elle peut permettre de mieux apprécier sa propre communauté, sa propre société, sa propre « culture », peut être également source de frustration personnelle, comme c'est souvent le cas des Colombiens face aux Français, et en particulier pour les professeurs de FLE non natifs.

\subsection{La figure du locuteur natif dans l'enseignement des langues en Colombie}

Dans le contexte de l'apprentissage du FLE en Colombie, le locuteur « natif » idéal reste le Français de la métropole. En effet, le français académique est perçu comme celui parlé en France, les autres expressions de la francophonie n'étant pas considérées comme légitimes (Rincón Restrepo, 2016 : 327). Le statut de la langue française varie selon qu'elle est langue maternelle, officielle, d'usage, de culture et si elle est parlée par une majorité ou une minorité de locuteurs, selon Lise Gauvin (2008: 28). Les « francophones » des zones où elle n'est pas langue maternelle ou est une langue minoritaire sont vus comme des usagers de la langue de second plan. Jusqu'à la première moitié du XXe siècle, on reconnaissait seulement une langue française, celle de la France, standardisée par le français parisien bourgeois de l'époque de la Révolution française. Ainsi, le français des autres régions du globe était considéré comme dialecte : celui du Canada était vu comme un mauvais mélange entre anglais et français, par exemple, et le français des anciennes colonies est souvent vu comme une imitation du véritable français. Par ailleurs, la France accepte encore mal que les identités nationales ne soient pas univoques, comme le dit Charles Bonn (2008 : 45), peut-être à cause d'une tradition jacobine 
postulant l'unité de la langue. Si les francophones de la « périphérie » ne sont pas reconnus comme des véritables «natifs » en Colombie, la situation est encore pire pour les enseignants « non natifs ». Ils sont souvent soupçonnés de ne pas maitriser « parfaitement » la langue qu'ils enseignent, de ne pas avoir le niveau d'un Français et d'être, tout simplement, de mauvais professeurs.

Si le français hexagonal est perçu en Colombie comme " authentique ", c'est parce que le contact avec la francophonie, au moins jusqu'au début du XXI siècle, y a souvent été assuré en exclusivité par des acteurs hexagonaux (Rincón Restrepo, 2016 : 328). L’Amérique latine en général est en effet l'une des régions du monde où la prégnance de la culture française est la plus forte, comme le fait remarquer Silva (2011 : 6). Ainsi, il est compréhensible que le français de France soit évoqué comme modèle dans l'enseignement du FLE, où l'on cherche à faire apprendre le français hexagonal selon une normativité linguistique pédagogique, en argumentant qu'elle permettrait de structurer plus facilement le savoir.

\subsection{Le statut du français en Colombie et la formation des professeurs de FLE: une autre source d'insécurité}

En Colombie, le français a actuellement le statut de deuxième langue étrangère, mais occupa longtemps une première place incontestable. C'est dans la période de l'après-guerre que le français commence véritablement à perdre de son influence face à l'anglais, lorsque la mise en place de lois éducatives se conforme à des pressions géopolitiques. Le français, dont l'enseignement devient peu rentable, disparait ainsi progressivement du secondaire, laissant l'anglais comme seule langue étrangère obligatoire (Rincón-Restrepo, 2020 : 50). La propagation de cette langue et de la culture américaine accompagne l'accroissement du commerce international, le développement des moyens d'information et les avancées scientifiques et techniques, qui engendrent des bouleversements économiques, sociaux et culturels, ainsi que des changements dans les mentalités.

Dans les années 1990, le gouvernement colombien reconnait dans la Nouvelle Constitution de 1991 le caractère multilingue et pluriculturel du pays. Malgré cela, il promeut essentiellement un bilinguisme espagnol-anglais dans le secteur éducatif, comme l'illustre le Programme national de bilinguisme 2004-2019 (PNB). La diversité linguistique est donc encore entendue comme une simple dualité entre la langue officielle et une seule langue étrangère. Le manque d'harmonisation des politiques linguistiques dans le domaine éducatif conduit à l'absence de langues étrangères autres que l'anglais dans les programmes de formation des enseignants des langues, et à une représentation très réduite des langues nationales autres que l'espagnol' .

7. En Colombie, $98 \%$ de la population parle espagnol, le reste parlant des langues nationales ou des langues créoles. 
Les tensions politiques se manifestent alors dans les universités colombiennes qui décident de réformer, voire de supprimer, les formations de professeurs de FLE. Le français perd complètement sa place dans l'enseignement secondaire vers la fin du XX e siècle et lutte pour garder un espace dans la formation universitaire des professeurs de cette langue.

Au début du XXI e siècle, les entités en charge de la validation de cette formation de professeurs de LE décident alors d'annuler les épreuves de langues autres que l'anglais de l'examen d'État (pruebas Saber Pro) à la fin des licences de formation universitaire ${ }^{8}$. La compétence d'un professeur de LE ne s'évalue ainsi qu'en anglais (en français si la formation est spécifique dans cette langue9) ce qui nuit à la qualité de la formation et qui peut, à long terme, engendrer une insécurité linguistique chez les professeurs, qui se sentent mal préparés à enseigner la langue française, perçue en Colombie comme une langue difficile et sophistiquée nécessitant de longues études pour être maitrisée.

Actuellement, les universités colombiennes, désireuses d’apparaitre en bonne place dans les classements internationaux, concentrent leurs programmes de formation des professeurs de LE sur la formation en anglais et délaissent celle dans d'autres LE, par exemple en accordant moins d'heures aux cours de littérature et culture françaises, ou en accordant peu de budget au recrutement de professeurs de FLE qualifiés. Ainsi, en 2015, le ministère de l'Éducation, après analyse des résultats des examens Saber Pro des années précédentes, a décidé de supprimer la formation en français dans des licences octroyant un double ou triple titre par diplôme, car les étudiants y obtenaient des résultats plus médiocres en anglais que ceux des licences octroyant un seul titre en cette langue. Cette décision n'a toutefois pas encore été appliquée, face à l'opposition d'une partie du corps enseignant, car elle pourrait entrainer la disparation presque totale du français dans le cadre académique universitaire, qui ne serait alors enseigné au niveau licence que dans deux universités du pays.

Pour l'instant, les victimes de ces politiques éducatives sont les étudiants de ces licences de formation de professeurs de LE, qui devenus enseignants ressentiraient de l'insécurité à l'idée d'avoir reçu une formation médiocre en langue et culture françaises, ce qui est, de fait, le cas. Dans une étude réalisée en 2014 en Colombie (Rincón-Restrepo, 2016), les étudiants dans des licences de formation de futurs professeurs de FLE octroyant un double titre anglais/français, estiment avoir reçu une meilleure formation en anglais qu'en français.

Néanmoins, ces dix dernières années, les entités chargées de la diffusion de la langue française ont commencé à « lutter » avec plus de force contre cette marginalisation du français dans les politiques linguistiques colombiennes. De nouveaux accords entre les institutions éducatives permettent une

8. Examen que doivent passer tous les étudiants à la fin de leur licence (BAC +5 en Colombie). Dans le cas des licences de formation de professeur de LE, l'examen Saber PRO évalue la connaissance pédagogique, la connaissance en didactique des LE, la connaissance linguistique en anglais et la connaissance de la problématique éducative en Colombie des futurs enseignants.

9. Parmi les 32 universités publiques et 49 privées du pays, 16 offrent une licence en formation initiale pour les professeurs de français. Parmi ces 16 programmes, deux proposent une formation exclusivement en français. 
mobilité des étudiants colombiens vers la France et le Canada, et Campus France, une agence gouvernementale française travaillant sur la promotion de l'éducation supérieure et la mobilité internationale née en 2006, est chargée de gérer toutes les démarches des étudiants désirant poursuivre leurs études en France pour compléter leur formation. Ces efforts ont permis, ces dernières années, un nouvel essor de l'enseignement du français en Colombie, principalement dans le secteur de l'enseignement privé dans des instituts des langues. Ils reflètent, en grande partie, une prise de conscience des pays européens, qui œuvrent avec une énergie nouvelle à diffuser leurs langues dans le monde. Le français, en tant que deuxième langue étrangère la plus apprise en Colombie et seule capable de faire contrepoids à l'anglais, joue un rôle moteur dans cette promotion de la diversité linguistique.

Par ailleurs, l'enseignement obligatoire du français dans les collèges et lycées en Colombie va être réintroduit. En 2010, le Mémorandum d'entente pour l'enseignement du français dans les institutions éducatives publiques en Colombie a été signé entre l'Ambassade de France en Colombie, l'Alliance colombo-française et le ministère de l'Éducation nationale. Ce mémorandum est le résultat d'un projet conduit depuis 2008 par un groupe d'universités publiques et par l'Association de professeurs de français (ACOLPROF), l'Alliance colombo-française et l'Ambassade de France, qui, après plusieurs années d'absence de cette langue dans les institutions publiques du pays, souhaitent que cet enseignement réintègre les programmes de formation (González, 2010). Le ministre français de l'Éducation nationale, Jean-Michel Blanquer, a également exprimé cette volonté lors de sa visite en Colombie en septembre 2019.

Dans ce mémorandum, toutes les parties considèrent la création des programmes éducatifs linguistiques et culturels indispensables au développement et à l'apprentissage de langues autres que l'espagnol. Pour sa part, l'Ambassade française en Colombie s'engage à élaborer et à mettre en place un programme suivant les orientations pédagogiques devant être établies avec le ministère et l'Alliance française; à coordonner la participation des organismes français dont l'alliance est considérée pertinente pour la mise en marche du programme; à contribuer à l'organisation, la préparation et la convocation d'événements académiques favorisant la réflexion et l'échange d'expériences en relation avec le programme. Ainsi, le français a commencé à être réintroduit dans l'éducation secondaire depuis 2012 dans quelques institutions, qui servent donc d'étude pilote.

\section{Conclusions}

L'insécurité linguistique chez les enseignants non natifs de FLE en Colombie provient de sources diverses, et rejoint celle ressentie par d'autres professeurs de LE non natifs dans le monde, comme le démontrent diverses études. D’après les catégories proposées par Calvet, ils ressentent principalement une insécurité identitaire, liée aux préjugés et aux représentations que se font les enseignants de langues du professeur « natif » et de ce qu'est un francophone, et une insécurité formelle, car ils considèrent que leur pratique linguistique est souvent non conforme aux normes. Cela est notamment lié à l'idée d'avoir reçu une formation moins bonne en français qu'en anglais dans les licences 
de formation de futurs de professeurs de FLE des universités colombiennes. Ces licences sont sujettes aux politiques linguistiques et éducatives établies au détriment des licences autres que celles liées à l'enseignement de l'anglais. S'y ajoute également la difficulté de mettre en pratique, dans la vie courante, leurs connaissances en LE.

Pour réduire le sentiment de vulnérabilité issu de l'insécurité identitaire, il serait bénéfique de revoir la formation des futurs professeurs de FLE dans ces programmes universitaires et de remplacer le paradigme actuel par une didactique du plurilinguisme. Il convient donc de dépasser la dualité «natif »/« non natif » et de redéfinir les objectifs de l'enseignement d'une LE, encore très centré sur un cadre "monolingue », afin de mettre les enseignants « non natifs » davantage en confiance. Aujourd'hui, l'objectif de l'apprentissage d'une langue n'est plus d'imiter un locuteur modèle ou idéal, mais d’apprendre peu à peu la langue et la culture qu'elle véhicule. Ainsi :

La pleine prise en compte du caractère profondément «pluri » de l'apprentissage des langues nécessite une rupture radicale avec l'enseignement traditionnel de type « monolingue/monoculturel » des langues et avec la formation tout aussi « monolingue/monoculturelle » des enseignants de langue(s). (Derivry-Plard, $2006: 107)$

Il faut par ailleurs accepter que, comme le démontrent plusieurs études, un natif quelconque ne peut remplacer un professeur de langue formé pour enseigner une LE (Cuq, 2003 : Torres-Castillo, 2018 : 247), mais devrait plutôt être considéré comme un « informateur » et comme « une ressource d'apprentissage ». L'enseignant doit alors guider, donner des pistes et fournir des stratégies d'apprentissage aux étudiants pour qu'ils deviennent compétents en LE, ce qui ne requiert pas d'être natif, mais d'obtenir de solides compétences d'enseignement. D'où la nécessité de formations de qualité. L'idée de former des individus avec une « maitrise parfaite » de la langue cible doit être oubliée, au profit d'une vision plus dynamique de la langue et de son processus d'apprentissage. Cette logique traditionnelle nous empêche de voir, comme le dit Torres-Castillo (2018: 383) que l'apprenant de LE, ici le professeur de LE, est à la fois un «natif » et un «non natif », et donc un locuteur plurilingue.

Quant à l'insécurité formelle, il est nécessaire de soutenir les licences de formation pour les futurs professeurs de LE dans les universités, notamment celles octroyant un double titre - professeur d'anglais et de français langue étrangère -, en menant une réflexion sur les transversalités didactiques entre les deux langues, car si ces programmes disparaissent, comment seront formés les futurs professeurs de FLE requis lorsque le français sera réintroduit dans le secondaire ? La création de programmes uniquement consacrés à la formation de professeurs de français n'est pas économiquement viable dans l'immédiat. À Bogotá, une formation de ce type peut attirer un nombre suffisant d'étudiants, puisqu'il existe un marché relativement vaste pour les diplômés. Ce pourrait être le cas aussi à Medellín et à Cali, mais pas dans le reste du pays.

Enfin, une nouvelle légitimité doit également être reconnue à l'enseignement de langues autres que l'anglais dans les secteurs du secondaire et universitaire, en accord avec les préconisations du 
CECR (Cadre européen commun de référence) selon lesquelles un apprenant ne doit pas uniquement maitriser une seule langue cible, mais être compétent dans plusieurs langues à différents degrés. 


\section{Références}

Benamar, Rabéa (2014), « La langue maternelle, une stratégie pour enseigner/apprendre la langue étrangère », Multilinguales, n³, p. 139-158.

Bonn, Charles (2008), «Pour un comparatisme français ouvert à la francophonie et aux métissages culturels : plaidoyer en forme de polémique », dans Ingse Skattum et Karin Holter (dir.), La francophonie aujourd'hui : réflexions critiques, Paris, L'Harmattan, p. 41-48.

Bourdieu, Pierre (1982), Ce que parler veut dire : l'économie des échanges linguistiques, Paris, Fayard.

Calvet, Louis-Jean (1993), La sociolinguistique, Paris, Presses universitaires de France.

Candelier, Michel et Véronique Castellotti (2013), "Didactique(s) du(des) plurilinguisme(s) », dans Jacky Simonin et Sylvie Wharton (dir.), Sociolinguistique du contact : dictionnaire des termes et concepts, Lyon, ENS Éditions, p. 179-221.

Causa, Maria (2002), L'alternance codique dans l'enseignement d'une langue étrangère : stratégies d'enseignement bilingues et transmissions de savoirs en langue étrangère, Bruxelles, Peter Lang.

Cook, Vivian (2016), « Where Is the Native Speaker Now ? », TESOL Quarterly, vol. 50, nº 1, p. 186-189.

Coulmas, Florian (1981), A Festschrift for native speaker, La Haye, De Gruyter Mouton.

Cuq, Jean-Pierre (dir.) (2003), Dictionnaire de didactique du français langue étrangère et seconde, Paris, CLE International.

Davies, Alan (2003), The native speaker: Myth and reality, Clevedon, Multilingual Matters.

Derivry-Plard, Martine (2003), Les enseignants d'anglais "natifs » et " non-natifs » : concurrence ou complémentarité de deux légitimités, thèse de doctorat, Université Paris 3/INALCO.

Derivry-Plard, Martine (2006), "Les enseignants "natifs" et "non-natifs" de langue(s) : catégorisation linguistique ou construction sociale? », Travaux de didactique du FLE, n55, p. 100-108.

Derivry-Plard, Martine (2008), « Comment les étudiants se représentent-t-ils leurs enseignants de langue étrangère ? », dans Pierre Martinez, Danièle Moore et Valérie Spaëth (dir.), Plurilinguismes et enseignement: identités en construction, Paris, Riveneuve, p. 141-152.

Francard, Michel, Joëlle Lambert et Françoise Berdal-Masuy (1993), L'insécurité linguistique en Communauté française de Belgique, Bruxelles, Service de la langue française de la Communauté française Wallonie-Bruxelles.

Galisson, Robert (1986), "Éloge de la didactologie/didactique des langues et des cultures (maternelle et étrangères) - (D/DLC) », Études de Linguistique Appliquée, n 64, p. 39-54.

Gauvin, Lise (2008), «Situations des littératures francophones : à propos de quelques dénominations », dans Ingse Skattum et Karin Holter (dir.), La francophonie aujourd'hui : réflexions critiques, Paris, L'Harmattan, p. 27-39. 
González, Liliana (2010), « Hacia una revolución francesa en la investigación sobre la didáctica del francés en Colombia », Signo y pensamiento, vol. 29, n57, p. 496-504.

Haugen, Einar (1962), « Schizoglossia and the Linguistic Norm », Monograph Series on Languages and Linguistics, n 15, p. 63-69.

Love, Nigel (2009), « Science, language and linguistic culture », Language \& Communication, vol. 29, $n^{\circ} 1$, p. 26-46.

Muni Toke, Valelia (2010), «La linguistique légale à la recherche du locuteur natif : de la détermination de l'origine des demandeurs d'asile», Langage et société, n 132, p. 51-75.

Muni Toke, Valelia (2013), «Le locuteur natif et son idéalisation : un demi-siècle de critiques », Histoire Épistémologie Langage, vol. 35, n², p. 5-15.

Paikeday, Thomas M. (1985), The native speaker is dead! An informal discussion of a linguistic myth with Noam Chomsky and other linguists, philosophers, psychologist, and lexicographers, Toronto/ New York, Paikeday Publishing.

Planet, Cathy (2014), « Natifs et non natifs dans l'enseignement des langues-cultures étrangères : genèse, aspects et perspectives d'un conflit de légitimités », Revista electrónica de Investigación Educativa Sonorense, $n^{\circ}$ 16, p. 86-96, disponible sur https://rediesonorense.files.wordpress. com/2014/09/revista-no-16.pdf.

Rampton, Ben (1990), «Displacing the 'native speaker': expertise, affiliation, and inheritance », ELT Journal, vol. 44, n², p. 97-101.

Rincón-Restrepo, Claudia (2016), Didactique du texte littéraire dans l'enseignement universitaire de français en Colombie : histoire, actualité et perspectives, thèse de doctorat, Paris, Université Sorbonne Nouvelle.

Rincón-Restrepo, Claudia (2020), "L'enseignement du français dans l'espace universitaire en Colombie : deux cents ans d'histoire, mais quel avenir? ", Synergies Chili, nº 16 (Du passé vers l'avenir: innovations et nouvelles compétences pour l'apprenant-citoyen du XXI siècle, sous la dir. de Marie-Noëlle Antoine et Isabel Alvarado Gutierrez), p. 49-62.

Roussi, Maria (2009), L'insécurité des professeurs de langues étrangère non natifs : le cas de professeurs grecs de français, thèse de doctorat, Paris, Université Sorbonne Nouvelle.

Silva, Haydée (2011), "Langues et territoires : le statut du français en Amérique latine », Cahier de la Chaire Senghor de la francophonie de l'Université du Québec en Outaouais, n³, disponible sur https://uqo.ca/docs/10063.

Skutnabb-Kangas, Tove et Teresa McCarty (2018), « Key concepts in bilingual education: ideological, historical, epistemological, and empirical foundations », dans Jim Cummins et Nancy Hornberger (dir.), Encyclopedia of Language and Education, vol. 5 (Bilingual Education), $3^{e}$ éd., New York, Springer, p. 3-17. [1'é éd., 1997.] 
Torres-Castillo, Claudia (2017), Culturas lingüísticas : el habitus en un mundo plurilingüe, thèse de doctorat, México, Universidad Autónoma del Estado de México.

Torres-Castillo, Claudia (2018), Enseignement du français, altérités et contacts de langues : imaginaires de professeurs mexicains, thèse de doctorat, Tours, Université de Tours.

Urbain, Jean-Didier (1982), «La langue maternelle, part maudite de la linguistique? », Langue française, n 54, p. 7-28. 


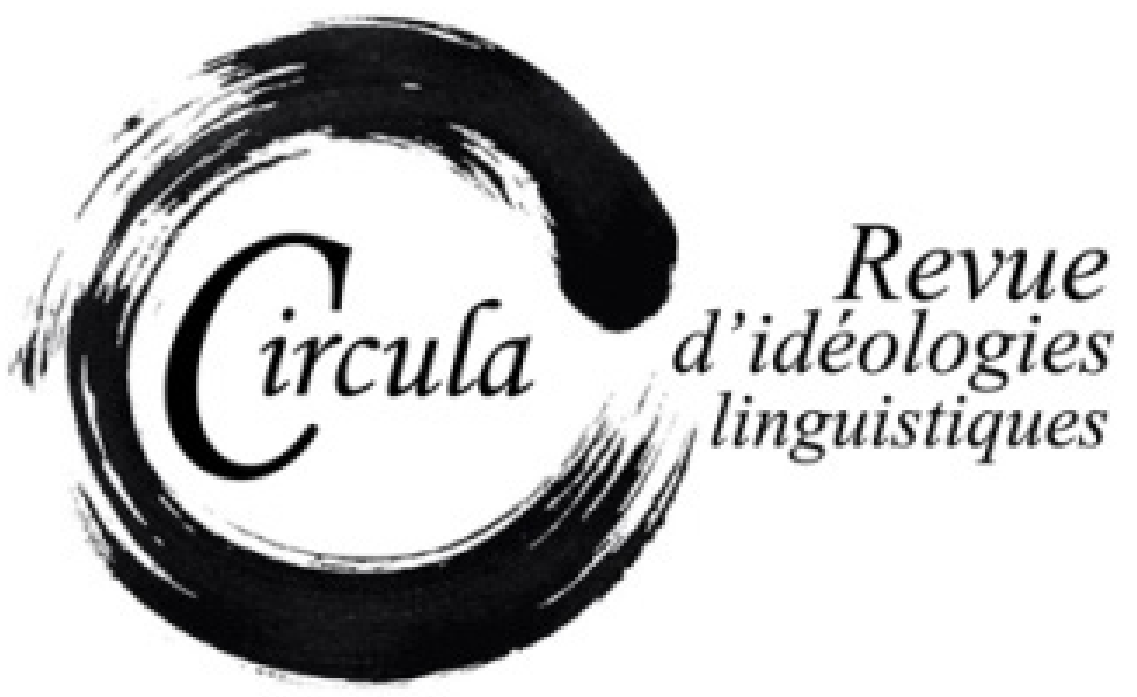

TItTRE: VALENTIN FEUSSI ET JOANNA LORILLEUX (DIR.), (2020), (IN)SÉCURITÉ LINGUISTIQUE EN FRANCOPHONIES: PERSPECTIVES IN(TER)DISCIPLINAIRES, PARIS, L'HARMATTAN, COLL. «ESPACES DISCURSIFS 》, 452 P. [ISBN : 978-2-343-20806-0]

Auteur: Cynthia BARrÉ-Benoit, Université de SHERBRooke

RevUe: CIRCULA, NUMÉRO 12 : LA VULNÉRABILITÉ LINGUISTIQUE

DiRECTRICE: CLAUDIA TORRES CASTILLO

PAGES: $197-204$

ISSN: 2369-6761

URI: HTTP://HDL.HANDLE.NET/11143/18449 


\section{Valentin Feussi et Joanna Lorilleux (dir.), (2020), (In)sécurité linguistique en francophonies : perspectives in(ter) disciplinaires, Paris, L'Harmattan, coll. "Espaces discursifs ", 452 p. [ISBN : 978-2- 343-20806-0]}

Cynthia Barré-Benoit, Université de Sherbrooke Cynthia.Barre-Benoit @ USherbrooke.ca

Cet ouvrage collectif, dirigé par Valentin Feussi et Joanna Lorilleux, vise à jeter de nouveaux éclairages sur la notion d'insécurité linguistique (désormais IL) ainsi que son corollaire, la sécurité linguistique (désormais SL), en tenant compte de la pluralité des expériences francophones qui caractérise notre époque. II rassemble les travaux présentés lors du colloque Les «francophones » devant les normes: 40 ans après Les Français devant la norme. L'(in)sécurité linguistique aujourd'hui : perspectives in(ter)disciplinaires, tenu à l'Université de Tours en juin 2018. La parution de ce volume rappelle ainsi que Nicole Gueunier, coauteure des Français devant la norme, a été la première à ériger l'IL en objet d'étude dans l'espace francophone et a joué un rôle déterminant dans la recherche sur ce phénomène. S’inspirant des travaux variationnistes menés au sein d'autres communautés linguistiques, elle a su innover par rapport à ceux-ci en prêtant attention non seulement aux productions linguistiques des locuteurs, mais également à leurs discours métalinguistiques. Elle a ainsi ouvert la voie à plusieurs chercheurs qui ont, par la suite, laissé leur marque. Notamment, Pierre Bourdieu (1987), Michel Francard (1993) et Louis-Jean Calvet (1999) se sont intéressés aux rapports inégalitaires entre les langues (ou variétés de langues) en situation de contact, remarquant une association entre l'auto-dévalorisation des pratiques linguistiques et la domination/minorisation subie au quotidien. Plus récemment, des chercheurs comme Didier de Robillard (2008), Aude Bretegnier (2009) et Annette Boudreau (2016) ont montré que les manifestations de l'ISL fluctuent en fonction des paramètres de chaque interaction. Ce caractère hétérogène et dynamique doit être compris en lien avec la trajectoire sociobiographique et langagière des personnes, y compris celle du chercheur. Si ces jalons théoriques et méthodologiques ont contribué à faire avancer la réflexion, force est de constater que l'IL, fréquemment décrite comme un « sentiment et un malaise » (p. 12) ou une « quête de légitimité » (p. 38), demeure relativement difficile à circonscrire. Alors que ce phénomène n'avait plus fait l'objet d'une publication d'envergure depuis plusieurs années, Valentin Feussi et Joanna Lorilleux le réhabilitent au sein de la recherche actuelle en sociolinguistique, tout en faisant appel 
aux contributions d'autres disciplines. Cet ouvrage, qui contient 31 chapitres, est articulé autour de quatre axes thématiques: " expériences francophones », « réflexions épistémologiques », " questionnements didactiques » et « perspectives littéraires ».

Dans le chapitre introductif, Valentin Feussi rappelle que si le phénomène de l'IL n'est pas exclusif à l'espace francophone, il en est néanmoins caractéristique. L'idéologie normative qui a historiquement accompagné la diffusion du français engendre, encore aujourd'hui, des inégalités là où cette langue est parlée. Derrière le projet d'une francophonie soi-disant universaliste et engagée en faveur de la pluralité, se dissimulent des tensions sociopolitiques, identitaires et culturelles qui trahissent un rapport conflictuel au français. Dans les anciennes colonies aussi bien que dans l'Hexagone, le français a été imposé à l'ensemble de la population comme langue de scolarisation et règne dans les toutes les sphères officielles : administration, justice, échanges internationaux, médias, etc. Conséquence des efforts liés à son institutionnalisation et sa diffusion internationale, la variété standard du français demeure fortement associée à un profit de distinction, notamment dans les sociétés fortement plurilingues. Pour poursuivre cette réflexion initiale, Michel Francard et Aude Bretegnier proposent une rétrospective de leurs recherches respectives sur l'IL, en les resituant dans leur contexte institutionnel. Premièrement, Michel Francard souligne la rupture qu'a opérée le groupe de recherche VALIBEL avec l'approche quantitative qui prévalait en sociolinguistique depuis Labov, en déplaçant leur attention sur les discours épilinguistiques et en observant l'IL « dite » plutôt qu' "agie »1. Principalement situées en Belgique francophone, les recherches de VALIBEL ont aussi pointé le rôle de l'école dans la transmission et la valorisation de la norme parisienne auprès des francophonies «périphériques». Aude Bretegnier soulève quant à elle tout le paradoxe qui consiste à traiter l'IL, définie en tant que "sentiment », comme une réalité immuable. Elle insiste sur son caractère interactionnel, l'envisageant « non pas en rapport exclusif à une langue-variété normative, mais dans la relation conflictuelle vécue entre des langues inégalement légitimées » (p. 39). La situation réunionnaise, où le créole est investi comme symbole identitaire et utilisé pour exclure le groupe francophone dominant de certaines sphères de socialisation, démontre toute cette complexité.

La première section thématique de l'ouvrage, consacrée aux expériences francophones, expose une dizaine de recherches ancrées dans divers territoires où la langue française revêt une importance historique, sociale et symbolique. Elle met en lumière différentes formes d'appropriation de la langue française qui traduisent, tour à tour, une certaine insécurité ou une certaine sécurité linguistique. Annette Boudreau ouvre la marche en se penchant sur les représentations du locuteur légitime/ illégitime qui circulent en Acadie et relève l'importance du lieu et de la honte comme éléments constituants de l'IL. Toujours au Canada francophone, Wim Remysen s'intéresse à l'insécurité linguistique « agie » des Québécois et analyse l'accommodation de leurs pratiques linguistiques face à des interlocuteurs français. Puis, quatre textes nous entraînent en Afrique de l'Ouest, terreaux fortement multilingues où le français, doté d'une aura de prestige, est utilisé pour les fonctions hautes de la société et est transmis par l'institution scolaire. Abou Bakry Kébé discute du bouleversement des

1. Cette distinction a été posée par Marie-Louise Moreau (1996). 
dynamiques sociolinguistiques dans la vallée du fleuve Sénégal, provoqué par le retour des émigrés après un séjour en France. L'article de Béatrice Boutin Akissi et Oreste Floquet analyse les évaluations épilinguistiques et les explications métalinguistiques de collégiens en Côte d'Ivoire et au Niger et remet en question le stéréotype selon lequel toutes les populations d'Afrique francophone se trouvent en situation d'IL par rapport au français. Croyance Pistis Mfwa contraste l'insécurité linguistique vécue par des ressortissants des deux Congo habitant en France, démontrant que les rapports individuels au français se construisent en relation au récit historique et sociopolitique ayant accompagné l'adoption du français comme langue officielle dans chaque communauté. Rassoul Khadimou Thiam s'interroge sur les conséquences pernicieuses du poids de la norme sur l'appropriation du français au Sénégal ainsi que sur l'investissement du wolof comme langue véhiculaire dans ce pays. De son côté, Tassadit Toumert se questionne sur les facteurs qui génèrent de l'IL chez les étudiants algériens inscrits dans les universités françaises, tels que le poids de la norme scolaire, les jugements dépréciatifs des jeunes Français à leur égard et l'idéalisation de la figure du locuteur natif. Dans la même veine, Véronique Fillol se penche sur l'IL vécue par des futurs maîtres de français en NouvelleCalédonie, où le français cohabite avec les langues kanak, et postule que l'IL peut être dépassée en prêtant attention aux auto biographies sociolangagières. Investigant le rapport des créolophones de Guadeloupe à leur langue maternelle, Sally Stainier soutient que les politiques en faveur de la standardisation et l'enseignement du créole ont contribué à façonner une image fantasmée du locuteur natif, l'appropriation du créole étant souvent malaisée en raison de la préséance du français à l'école. Enfin, cette section se conclut par une contribution de Clara Mortamet qui s'interroge sur les conditions permettant (ou non) de considérer l'hypercorrection orthographique comme une manifestation valide de l'ISL.

Le second axe est consacré aux questionnements épistémologiques. Les trois premières contributions jettent les bases d'une approche « délingualisée » (p. 221) de l'ISL. Celle-ci nous enjoint à concevoir l'IL au-delà de ses manifestations linguistiques et épilinguistiques, pour s'intéresser également à ses aspects plus insaisissables, qui relèvent de l'imaginaire. Une telle perspective passe par l'affranchissement du modèle positiviste - qui a traditionnellement traité l'ISL comme un phénomène objectivable - et la mise en valeur le sens qui émerge des récits de vie. D’abord, dans le sillon de la théorie phénoménologique de Humboldt, Valentin Feussi considère que « la langue émane d'un arrière-plan dynamique et inséparable de la vie quotidienne des individus » (p. 191). Celui-ci aborde l'IL comme une manière d'être au monde qui se construit dans l'interaction. Pour sa part, Jean-Marie Klinkenberg voit dans l'IL un processus d'ajustement qui naît d'une tension face à l'altérité, et dont les retombées peuvent être positives ou négatives. Il prône une « dépsychologisation » (p. 201) de l'IL afin de se décentrer de l'individu et se focaliser sur les structures productrices d'inégalités, avec les normes langagières au premier chef. Mylène Lebon-Eyquem s'attarde ensuite à la notion d'historicité. Pour celle-ci, tout chercheur ayant des prétentions de scientificité doit expliciter sa trajectoire, car l'expérience personnelle constitue le socle interprétatif de l'IL. Réticente face au terme d'insécurité linguistique, trop étroitement associé à un état de domination, elle argue que ce sentiment est parfois mobilisateur, lorsqu'il incite une communauté à revendiquer son identité par 
exemple. Les chapitres suivants discutent d'enjeux reliés à l'IL dans des contextes moins souvent étudiés. Réfléchissant à l'évaluation des écrits scientifiques, Joanna Lorilleux avance que les attentes institutionnelles implicites quant au sens des termes employés et à la forme du texte peuvent générer une certaine « insécurité langagière ». Moins associée à la maîtrise de la langue qu’aux codes qui entourent la réception et l'interprétation du discours, cette forme d'insécurité est susceptible d'inhiber la créativité de l'auteur souhaitant être publié. Ali Becetti s'approprie la démarche phénoménologico-herméneutique et démontre que ses propres représentations du français, héritées d'une éducation algérienne survalorisant la norme, influencent malgré lui les jugements qu'il porte sur les productions langagières des autres. Marc Debono réfléchit quant à lui à la tension entre sécurité et liberté qui caractérise la linguistique légale. Il évoque les dérives possibles de la « sécurisation » du sens dans cette discipline, où le contrôle des usages de la langue vient justifier certaines interventions judiciaires. Enfin, bouclant cette thématique, Isabelle Pierozak revisite l'ISL à l'aune du Monolinguisme de l'autre de Derrida. Il en ressort que toute personne entretient un rapport subjectif et hétérogène aux langues de son répertoire, façonné par son expérience du monde. Lorsqu'on la conçoit comme une disposition langagière mouvante relevant du spectre de la sensibilité, l'ISL ne peut être ni catégorisée, ni précisément nommée.

Le troisième axe met de l'avant des questionnements didactiques autour de l'ISL. Les contributions examinent notamment la traduction du couple sécurité/insécurité dans un contexte de formation ou d'évaluation, où la maîtrise de la langue revêt des enjeux importants. D’entrée de jeu, Véronique Castellotti rappelle le rôle central qu'exerce la norme en enseignement dans langues et pointe ses effets inhibiteurs pour l'appropriation du français par les migrants. Selon elle, un changement de paradigme misant davantage sur la compréhension/réception de la langue et intégrant la pluralité permettrait aux apprenants de regagner confiance en leurs moyens. Léa Courtaud s'intéresse aux formes d'IL éprouvées par les étudiants français durant leur parcours universitaire. Elle soutient qu'à côté de la maîtrise formelle de la langue, souvent mentionnée par les étudiants, une série d’implicites d'ordre disciplinaire, épistémologique ou culturel interviennent dans le sentiment et la capacité de réussite. Puis, un texte de Véronique Miguel Addisu et Evelyne Delabarre interroge les correspondances entre les pratiques de l'orthographe et les discours épilinguistiques chez les adultes lettrés, réaffirmant les liens entre la scolarisation et la prégnance de représentations linguistiques normatives. Dans l'article suivant, Alper Aslan aborde les aspects anxiogènes entourant la certification DELF. Il note que l'imposition de sujets qui sont trop éloignés de la culture et des intérêts des candidats, l'attitude intimidante de l'examinateur ou encore la conscience des enjeux socioprofessionnels liés à l'épreuve peuvent porter préjudice aux aspirants, malgré des compétences suffisantes en français. Explorant les représentations des migrants liées à leur accent étranger, Myriam Dupouy souligne la propension de ce public à associer le déclassement social avec des lacunes en français. En perte de statut, les migrants se compareraient ainsi à un locuteur natif fantasmé, au sommet de l'échelle linguistique et sociale. Emmanuelle Huver évoque quant à elle les dilemmes didactiques et identitaires d'étudiants tourangers se destinant à l'enseignement en Colombie-Britannique, dans un environnement francophone minoritaire. Elle démontre que des locuteurs détenteurs de la variété dominante, 
élevés dans une relative sécurité linguistique, peuvent aussi s'interrogent sur leur légitimité à représenter et transmettre la norme. Enfin, Qiu Shuming se penche sur la variation dans les manuels de français utilisés en Chine. Elle remarque que la diversité dans l'espace francophone y est dépeinte comme un fait marginal et anecdotique, ce qui ne permet pas de « conduire les apprenants à prendre en considération le fait qu’une langue puisse obéir à différentes normes » (p. 376).

Le quatrième et dernier axe engage des perspectives littéraires et s'intéresse aux manifestations de l'ISL chez les auteurs francophones qui vivent une certaine ambivalence linguistique et culturelle. Comme le montre Michel Ferrier, la remise en question de l'idéologie monolingue prend souvent la forme, dans la littérature postcoloniale, d'une «scène primitive » (p. 381) où se joue la rencontre avec l'altérité. Même dans les espaces considérés comme linguistiquement homogènes, tels que le Japon, des auteurs binationaux ou issus de l'immigration revendiquent leur dualité linguistique en entremêlant les systèmes graphiques. Pour Sylvie Dardaillon, l'écriture en français peut servir d'exutoire aux femmes maghrébines dont les voix, dans leur langue d'origine, sont parfois étouffées. Oscillant entre une pression de conformité face aux standards normatifs de la littérature française et une volonté de rompre avec ces injonctions, ces auteures utilisent leur insécurité comme moteur de création. Pierre Fandio rejoint ces propos en prenant l'exemple des humoristes camerounais, qui écorchent volontairement la grammaire et la prononciation du français pour produire un effet comique. Cette ironisation sur la difficulté à s’approprier la langue française au Cameroun reflète les inégalités statutaires qui existent entre les deux langues officielles (français et anglais) d'une part et entre les langues officielles et coloniales d'autre part. Enfin, en insistant sur l'hétérogénéité de la langue française, visible à travers la littérature, Myriam Suchet dénonce les frontières conceptuelles posées entre les langues et suggère de basculer vers un paradigme hétérolingue, qui accueille la diversité et l'instabilité. Elle élargit la réflexion au-delà du champ littéraire et linguistique en encourageant les collaborations in(ter)disciplinaires en recherche.

Suite à la lecture de cet ouvrage, on ne peut que saluer l'initiative de Valentin Feussi et Joanna Lorilleux de revisiter la notion d'ISL dans une optique de décloisonnement disciplinaire. Tout en reconnaissant l'apport des travaux qui ont cherché à décrire ce phénomène à partir de traces observables, plusieurs contributions rassemblées dans ce livre rompent avec le paradigme positiviste en s'attardant aux aspects plus intangibles de l'ISL. C'est toute la question de la «délingualisation » de cette notion qui se pose ici. En effet, plusieurs auteurs soulignent que si l'ISL n'est pas toujours dicible ou discernable par des signes linguistiques, les situations de contact entre des langues (ou variétés de langues) inégales entraînent toujours une résonnance sensible chez les locuteurs. En envisageant I'ISL comme un rapport aux langues (inter)subjectif dont le sens s'imbrique avec l'expérience du monde et l'historicité, ce volume enjoint également le chercheur à expliciter sa propre posture. Selon Didier de Robillard, un tel engagement est relié « au difficile choix éthique de cheminer à l'ombre de la science légitime, en l'assumant et en le revendiquant au cœur de ses propres écrits de recherche » (p. 445). Un autre mérite de ce volume, présenté comme son objectif de départ, est de parvenir à élargir les perspectives sur l'ISL en francophonie en diversifiant les contextes d'étude ainsi que les 
approches théoriques et méthodologiques. Prises dans leur ensemble, les contributions donnent à voir que l'expérience de l'IL (si l'on se résout à la nommer ainsi) rallie bon nombre de francophones, qu'ils soient locuteurs d'une variété dite " périphérique », qu'ils habitent un ancien territoire colonial où le français a conservé un rôle officiel et une position prestigieuse ou qu'ils aient appris le français aux suites de la migration. Le rapport complexe que ces locuteurs entretiennent au français (et plus précisément, à sa norme standard) peut certes être à l'origine de conséquences négatives (paralysie, sentiment d'illégitimité, dévalorisation, etc.), mais il peut également devenir moteur de création, d'action, de revendications. Alors qu'elles constituent des stratégies de négociation de la tension identitaire, culturelle et linguistique, ces externalités positives de l'IL sont rarement mises de l'avant par la recherche. Ce volume invite à de futures explorations en ce sens. 


\section{Références}

Boudreau, Annette (2016), À l’ombre de la langue légitime, Paris, Classiques Garnier.

Bretegnier, Aude (2009), « Sociolinguistique alter-réflexive : du rapport au terrain à la posture du chercheur », Cahiers de sociolinguistique, n 14, p. 27-42.

Calvet, Louis-Jean (1999), Pour une écologie des langues du monde, Paris, Plon.

Robillard, Didier de (2008), Perspectives alterlinguistiques, Paris, L'Harmattan, coll. «Espaces discursifs ».

Francard, Michel (1993), L'insécurité linguistique en Communauté française de Belgique, Bruxelles, Service de la langue française, coll. « Langue et société, 6 ».

Moreau, Marie-Louise (1996), «Insécurité linguistique: pourrions-nous être plus ambitieux? Réflexions au départ de données camerounaises, sénégalaises et zaïroises », dans Claudine Bavoux (dir.), Français régionaux et insécurité linguistique, Paris/Saint-Denis de la Réunion, L'Harmattan/ Université de la Réunion, p. 103-114. 


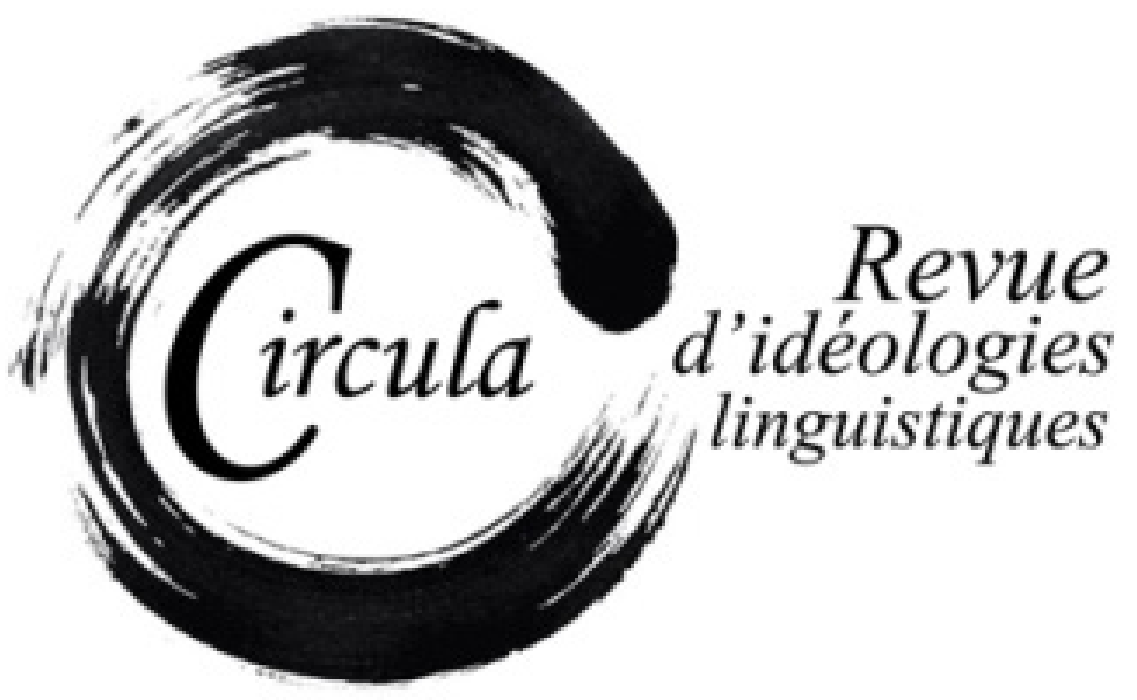

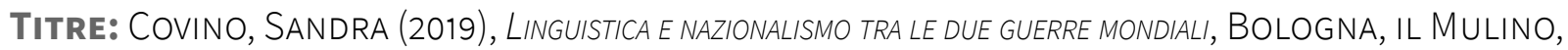
271 P. [ISBN: 978-88-15-28387-0]

Auteur: Maria Silvia Rati, Università per Stranieri “Dante AlighierI”

Revue: CIRCula, NumÉro 12 : LA VULNÉRABILITÉ LINGUISTIQUE

Directrice: Claudia TORRES CASTILLO

PAGES: $205-208$

ISSN: 2369-6761

URI: HTTP://HDL.HANDLE.NET/11143/18450 


\title{
Covino, Sandra (2019), Linguistica e nazionalismo tra le due guerre mondiali, Bologna, il Mulino, 271 p. [ISBN: 978-88-15- 28387-0]
}

\author{
Maria Silvia Rati, Università per stranieri “Dante Alighieri”, Reggio Calabria
} rati@ unistrada.it

Il volume presenta un'approfondita indagine sulle componenti ideologiche che, nella prima metà del Novecento, hanno condizionato le acquisizioni scientifiche delle filologie moderne, «il cui paradigma fondativo affonda le radici nella cultura romantica e nella correlata costruzione delle identità nazionali» (p. 11). Come ricorda più volte l'A., nello schema ideologico dello Stato nazione a lungo dominante nel contesto europeo è stato attribuito un ruolo centrale alla "lingua materna" e in particolare al suo modello istituzionale, e ciò ha sfavorito l'adozione di prospettive teoriche che si focalizzassero sui fenomeni di contatto e di mescolanza linguistica.

La ricostruzione delle vicende biografiche e dei punti di vista dei protagonisti del dibattito linguistico italiano ed europeo dei primi decenni del Novecento, portata avanti nei tre capitoli del volume, rafforza nel lettore la consapevolezza che le posizioni assunte dai diversi studiosi - e, quindi, anche i risultati scientifici a cui essi sono approdati - non possano essere considerate disgiuntamente dai condizionamenti esercitati dalla temperie politica dell'epoca a cui appartenevano (nazionalismo, patriottismo, irredentismo) e dalla militanza di ciascuno all'interno di un determinato schieramento (in particolare nell'epoca dei regimi totalitari).

Il primo caso esaminato è quello della strumentalizzazione politica delle tesi ascoliane sull'“unità ladina": la possibilità di considerare il ladino come un sistema autonomo rispetto alle varietà romanze cisalpine fu da un lato enfatizzata da Gartner, in accordo col disegno dello stato asburgico che mirava a rafforzare la fedeltà dei sudditi anche attraverso la valorizzazione della loro specificità linguistica, dall'altro negata, in Italia, da Salvioni, Monaci, Battisti. Secondo l'A., «è innegabile che alla componente scientifica degli scritti di Salvioni e di Battisti sulla questione ladina si accompagni un forte impegno politico, almeno sul piano della politica linguistica e culturale» (p. 43). Nella parte del volume in cui si affronta tale questione, un ampio spazio è dedicato alla figura di Carlo Battisti, il cui forte patriottismo, non esente da legami con l'irredentismo trentino, gli costò la mancata nomina a professore associato all'Università di Vienna. La ricostruzione della vicenda umana e intellettuale di Battisti, condotta anche con l'ausilio delle informazioni offerte da un recentissimo studio di Alessandro Parenti (che si fonda su un'ampia documentazione attinta dall'Archivio dell'Università di 
Vienna e dall'Istituto di studi per l'Alto Adige), dimostra, secondo l'A., quanto «anche l'ambiente accademico dei romanisti austriaci si fosse lasciato condizionare dalle infiltrazioni della politica nella sfera della ricerca scientifica» (p. 61). Trattando degli scritti di Battisti, che si schierò, anche, contro la teoria magnogreca di Rohlfs sulla grecità linguistica dell'Italia meridionale, l'A. inserisce un'interessante parentesi relativa alle obiezioni che furono portate dalla maggior parte dei linguisti italiani alla tesi di Rohlfs, e cita un'osservazione di Fanciullo che fa intendere come probabilmente questi studiosi posero in subordine gli aspetti linguistici della questione rispetto a quelli ideologici: «Negli anni Venti almeno - quando Trento e Trieste erano state appena "liberate" e la mancata cessione della Dalmazia faceva parlare di "vittoria mutilata" - l'eventualità di una mal avvenuta latinizzazione dell'Italia meridionale estrema poteva in effetti apparire una bestemmia» (p. 64).

Il secondo caso di strumentalizzazione ideologica analizzato nel volume è quello del riconoscimento dell'italianità - o meglio della "romanità" - delle parlate dell'Istria e della Dalmazia. L'appartenenza di queste parlate al dominio dei dialetti italiani era stata già postulata da Ascoli, che, tuttavia, era contrario all'utilizzazione di questa tesi per rivendicazioni di matrice irredentista. In séguito fu Matteo Bartoli a portare «il maggiore contributo alla costruzione ideologica, attraverso l'utilizzo di strumenti scientifici, del mito irredentista giuliano» (p. 65). Dall'analisi degli scritti di Bartoli e di altri linguisti dell'epoca (come Tolomei) emerge come, sia per il caso delle parlate dell'Alto Adige, sia per quello delle parlate dalmatiche, lo strumento scientifico utilizzato è stato il medesimo: il ridimensionamento e spostamento avanti nel tempo della portata dell'influsso germanico nel primo caso, di quello slavo nel secondo. Il presupposto ideologico che sta dietro questa operazione è, per l'A., il "mito della romanità": «Meriterebbe una ricerca a parte proprio l'influsso esercitato sulla linguistica italiana della prima metà del Novecento dal mito della romanità, che il Risorgimento aveva interpretato come premessa dell'Italia unita ma che prima e durante il ventennio fu utilizzato per giustificare pretese imperialiste $\mathrm{o}$ addirittura atteggiamenti razzisti» (p. 74).

L'A. mostra come in Bartoli perfino il concetto di prestigio linguistico, considerato da alcuni "il primo germe" del concetto di egemonia gramsciano, fosse condizionato da un forte pregiudizio nazionalistico e da un'impostazione teorica che risentiva di influssi fichtiani: così, l'alta permeabilità agli italianismi delle varietà slave di Istria e Dalmazia e la notevole disponibilità al bilinguismo degli slavi testimoniavano, per Bartoli, «un'autopercepita e riconosciuta inferiorità etnico-culturale da cui sarebbe scaturito un naturale consenso emulativo» (p. 79). L'atteggiamento ideologico di Bartoli ne viziò anche le ricerche sul veglioto: sono citati, a questo proposito, alcuni studi recenti di linguisti croati che, pur riconoscendo i meriti scientifici delle ricerche di Bartoli, lo accusano di aver occultato il carattere antico dell'interferenza slavo-romana, che aveva creato una "linguisticità indipendente". L'A. fa notare che, dal canto loro, i linguisti slavi spingono oggi per considerare l'istrioto non come appartenente al dominio italo-romanzo ma come un sistema romanzo a sé stante, «a conferma che il condizionamento ideologico e l'uso politico della linguistica sono fenomeni trasversali e persistenti nello spazio e nel tempo» (p. 84). 
Nel terzo capitolo entra in scena la figura di Clemente Merlo, di cui viene ricostruita, in particolare, la polemica con Bartoli relativamente al veglioto: secondo Merlo il dalmatico di Veglia andava considerato affine al ladino e al rumeno piuttosto che, come sosteneva Bartoli, ai dialetti abruzzesi e pugliesi. In questo caso l'A. sottolinea come lo stesso Bartoli «avesse lasciato trapelare la motivazione ideologica della prevalenza accordata alle "concordanze appennino-balcaniche"» (p. 97). I vari aspetti della concezione linguistica di Clemente Merlo su cui si sofferma il volume sono visti spesso in rapporto al pensiero di Ascoli; si evidenzia la fedeltà di Merlo ad Ascoli anche dopo la promulgazione delle leggi razziali, quando «Merlo non abiurò il suo culto ascoliano». Come sottolinea l'A., Ascoli era diventato un «padre scomodo del cui ricordo disfarsi e il richiamo al suo magistero una dichiarazione implicita di non allineamento ai dettami del regime. La circostanza può apparire una specie di beffa della storia, se si considera che, appena sei anni prima, l'eccessiva e perdurante influenza di Ascoli era stata indicata da Spitzer come un fattore che impediva alla linguistica italiana quel rinnovamento degli studi verificatosi in altri paesi europei» (p. 114).

Di grande interesse è l'Appendice del volume, in cui sono pubblicate e commentate le lettere inedite tra Hugo Schuchardt e Francesco D’Ovidio risalenti agli anni 1915-1921. Nel complesso, il carteggio offre una testimonianza significativa di come negli anni della Grande Guerra la stragrande maggioranza dei docenti universitari «mise le sue competenze al servizio delle rispettive cause nazionali [...] costituendo così una sorta di "terzo fronte" del terribile scontro in atto» (p. 133). Appare notevole soprattutto il fatto che Schuchardt, inizialmente aperto a valore del plurilinguismo e dell'integrazione tra lingue e culture diverse in coerenza con la vocazione dell'Impero asburgico, attuò, in séguito al coevo intensificarsi delle tensioni nazionalistiche, un cambio di prospettiva in direzione della concezione tradizionale delle lingue come emblemi dello Stato nazione. L'A. sottolinea quella che fu una vera e propria "regressione" nella concezione del linguista: "Di fronte al crollo di quel "grandioso esperimento" che era stato per Schuchardt l'Impero multinazionale asburgico, la sua reazione, invocando il principio wilsoniano dell'autodeterminazione dei popoli, recuperava sul piano teorico l'identificazione fichtiana dello Stato con la nazione, intesa in termini di identità collettiva e omogenea» (p. 175). Le lettere di D'Ovidio, pur animate da patriottismo, mostrano invece un superamento della concezione romantico-ottocentesca di nazione, come dimostra il fatto che, a proposito dei Còrsi, il filologo molisano ritenesse che l'identità linguistica italiana potesse conciliarsi con l'appartenenza dei parlanti a uno Stato diverso dall'Italia. 